\title{
Simulation Study of a Follow-On Gravity Mission to GRACE
}

by

\section{Bryant Loomis}

B.S., Hope College, 2003

M.S., University of Colorado at Boulder, 2005

A thesis submitted to the

Faculty of the Graduate School of the

University of Colorado in partial fulfillment

of the requirements for the degree of

Doctor of Philosophy

Department of Aerospace Engineering Sciences

2009 
This thesis entitled:

Simulation Study of a Follow-On Gravity Mission to GRACE written by Bryant Loomis

has been approved for the Department of Aerospace Engineering Sciences

R. Steven Nerem

George Born

Peter Bender

John Wahr

Scott Luthcke

Date

The final copy of this thesis has been examined by the signatories, and we find that both the content and the form meet acceptable presentation standards of scholarly work in the above mentioned discipline. 
Loomis, Bryant (Ph.D., Aerospace Engineering Sciences)

Simulation Study of a Follow-On Gravity Mission to GRACE

Thesis directed by Professor R. Steven Nerem

The Gravity Recovery and Climate Experiment (GRACE) has been providing monthly estimates of the Earth's time variable gravity field since its launch in March 2002. The GRACE gravity estimates are used to study temporal mass variations on global and regional scales, which are largely caused by a redistribution of water mass in the Earth system. The accuracy of the GRACE gravity fields are primarily limited by the satellite-to-satellite range-rate measurement noise, accelerometer errors, and temporal aliasing caused by un-modeled high-frequency variations in the gravity signal. Full numerical simulations are performed for a GRACE Follow-On mission (GFO) to determine if a future satellite gravity recovery mission with improved technologies will provide better estimates of time-variable gravity, thus benefiting many areas of Earth systems research.

Several GFO configurations are considered for this study. The first case considered is a two-satellite collinear pair similar to GRACE. The best-case two-satellite mission is equipped with an interferometric laser ranging system and a drag-free system in a lower altitude orbit. The laser ranging system improves the satellite-to-satellite rangerate measurement accuracy to $\sim 1 \mathrm{~nm} / \mathrm{s}$ as compared to $\sim 1 \mathrm{micron} / \mathrm{s}$ for GRACE Kband microwave ranging, and the drag-free system more accurately removes the nonconservative forces acting on the satellites than the GRACE on-board accelerometers. Two "hybrid" missions are also considered. One hybrid is the GRACE design where the $\mathrm{K}$-band ranging is replaced by the laser; and the other is a drag-free, low altitude scenario 
with K-band ranging. A comparison of simulated gravity estimates is an important design tool in selecting the most important technologies to be considered for a future mission. A method for local time variable gravity recovery through mass concentration blocks (mascons) is used to form simulated gravity estimates for Greenland and the Amazon region for three GFO configurations and GRACE.

Simulation results show that only modest improvement is realized for even the best-case two-satellite mission due to temporal aliasing errors. These errors are caused by high-frequency variations in the hydrology signal and imperfections in the atmospheric, oceanographic, and tidal models which are used to remove unwanted signal. The various sources of aliasing errors are investigated separately through a series of numerical simulations, showing that the leading cause of the errors is dependent on the considered region.

This work concludes that applying the updated technologies alone, will not immediately advance the accuracy of the gravity estimates. If the scientific objectives of a GFO require more accurate gravity estimates, then future work should focus on improvements in the geophysical models, and ways in which the mission design or data processing could reduce the effects of temporal aliasing. 


\section{Acknowledgements}

This work was supported by NASA Headquarters under the Earth System Science Fellowship Grant NGT5 and the NASA Instrument Incubator Program (IIP) through ESTO at NASA/GSFC.

I would like to thank my academic advisor, Dr. R. Steven Nerem, for providing the direction and supervision of my research. I also thank Dr. George Born, Dr. Peter Bender, Dr. John Wahr, and Scott Luthcke for serving as members of the PhD committee. Additionally, I thank Scott Luthcke and Dave Rowlands for providing the GEODYN and SOLVE software packages and spending a great deal of their time assisting me with a variety of problems.

Lastly, I would like to thank my amazing wife who has been very supportive throughout this entire process. 


\section{Contents}

\section{Chapter}

1 Introduction 1

1.1 Background and Motivation . . . . . . . . . . . . . . 1

1.2 GRACE Results . . . . . . . . . . . . . . . . . . 3

1.2 .1 Hydrology . . . . . . . . . . . . . . . . . . . . 4

1.2 .2 Oceanography . . . . . . . . . . . . . . 7

1.2 .3 Glaciology . . . . . . . . . . . . . . . . . . . 11

1.2 .4 Solid Earth Science . . . . . . . . . . . . . . . . . . . 13

1.2 .5 Geodesy . . . . . . . . . . . . . . . . . . . 14

1.2 .6 Errors . . . . . . . . . . . . . . . . . 15

1.3 Previous Investigation into a GRACE Follow-On Mission . . . . . . . 16

1.4 Research Overview . . . . . . . . . . . . . . . . . . . 18

2 The Earth Gravitational Field 21

2.1 Introduction . . . . . . . . . . . . . . . . . . . 21

2.2 Definition of the Geoid . . . . . . . . . . . . . . . . . . 21

2.3 Spherical Harmonics . . . . . . . . . . . . . . . . . . . . . . 23

2.4 Mascons . . . . . . . . . . . . . . . . . . . . . 26 
2.4 .1 Point mass and disk mascons . . . . . . . . . . . . . 28

2.4 .2 Lumped harmonic mascon technique . . . . . . . . . . . . 29

2.5 Averaging Kernels . . . . . . . . . . . . . . . . . . . . 31

2.6 Methods for Analyzing the Accuracy of Gravity Estimates . . . . . . . . 34

3 Simulation Models and Procedure 38

3.1 Introduction . . . . . . . . . . . . . . . . . . . . . . 38

3.2 Force Models . . . . . . . . . . . . . . . . . . . . . . . 39

3.3 Linear Estimation Theory . . . . . . . . . . . . . . . . . . . 50

3.4 Gravity Recovery Simulation Procedure . . . . . . . . . . . . . . 53

3.4.1 Numerical Simulation Procedure . . . . . . . . . . . . . . 54

3.4.2 General Acceleration Parameter Estimation . . . . . . . . 58

3.4.3 Orbit Refinement With Baseline Parameterization . . . . . . . . 60

3.4.4 Spherical Harmonic Estimation . . . . . . . . . . . . . . 61

3.4.5 Mascon Parameter Estimation . . . . . . . . . . . . . 62

3.5 Mission Orbital Parameters . . . . . . . . . . . . . . . 63

4 Sources of Error $\quad 67$

4.1 Introduction . . . . . . . . . . . . . . 67

4.2 Inter-satellite Measurement Errors . . . . . . . . . . . . . . . 67

4.2 .1 GRACE . . . . . . . . . . . . . . . 68

4.2 .2 GRACE Follow-On . . . . . . . . . . . . . . . . 69

4.3 Accelerometer Errors . . . . . . . . . . . . . . . . . . . . 70

4.4 Satellite Positioning Errors _. . . . . . . . . . . . . 73

4.5 Temporal Aliasing Errors . . . . . . . . . . . . . . . . . . . 74

4.6 Summary of GRACE and GFO Mission Configurations . . . . . . . . . 77 
5 Simulation Results and Error Analysis $\quad 79$

5.1 Introduction . . . . . . . . . . . . . . . . . . . . . . 79

5.2 Basin Definitions . . . . . . . . . . . . . . . . 80

5.2 .1 South America . . . . . . . . . . . . . . . 81

5.2 .2 Greenland . . . . . . . . . . . . . . . . . 81

5.3 Mascon Estimation Methods . . . . . . . . . . . . . . . 84

5.3.1 $1^{\circ} \times 1^{\circ}$ Mascon Grid: One Constraint Matrix . . . . . . . . . 85

5.3.2 $1^{\circ} \times 1^{\circ}$ Mascon Grid: Separate Land and Ocean Constraints . . 88

5.3.3 Uncorrelated Basin Mascons . . . . . . . . . . . . . . . . . . . . 91

5.3.4 $1^{\circ} \times 1^{\circ}$ Mascon Grid: Greenland Elevation Constraints . . . . . 94

5.3.5 Comparison of Mascon Constraint Scenarios . . . . . . . . . . 96

5.4 Simulated Gravity Estimates: Instrument Noise Only . . . . . . . . . . . 100

5.4 .1 South America . . . . . . . . . . . . . . . . 101

5.4 .2 Greenland . . . . . . . . . . . . . . . . . 106

5.4.3 Comparison of Mission Configurations . . . . . . . . . . . . 111

5.4 .4 Spatial Resolution Study . . . . . . . . . . . . . . . . . 122

5.5 Simulated Gravity Estimates: All Error Sources . . . . . . . . . . . . . 126

5.5 .1 South America . . . . . . . . . . . . . . . 126

5.5 .2 Greenland . . . . . . . . . . . . . . . . . . . 131

5.5.3 Comparison of Mission Configurations . . . . . . . . . . . 136

5.6 Effects of Temporal Aliasing . . . . . . . . . . . . . . . . . . 145

5.6.1 Satellite Altitude Sensitivity to Aliasing . . . . . . . . . . . . . . 145

5.6.2 Separation of AOD and Tidal Aliasing Effects . . . . . . . . . 154

5.6.3 Measuring the Effect of Hydrological Aliasing . . . . . . . . . 157

5.7 Mass Variation Estimates From Mascons and Averaging Kernels . . . . 159 
6 Conclusions 164

6.1 Summary and Conclusions . . . . . . . . . . . . . . . 164

6.2 Recommendations . . . . . . . . . . . . . . 166

$\begin{array}{ll}\text { Bibliography } & 168\end{array}$

\section{Appendix}

A Spherical Harmonics 176

B Fourier Transforms 182

$\begin{array}{ll}\text { C Spectral Density } & 185\end{array}$

D Satellite-to-Satellite Range and Range-rate Equations 186

D.1 Tracking Observables . . . . . . . . . . . . . . . 186

D.2 Measurement Partial Derivatives _ . . . . . . . . . . . . . . . 187

D.3 Perturbed Motion Equations _. . . . . . . . . . . . . . . 187

E Application of Hill's Equations 191 


\section{Tables}

\section{Table}

3.1 Force models used for numerical simulations. . . . . . . . . . . . . . . . 41

3.2 Mascon definitions. . . . . . . . . . . . . . . . . . 63

3.3 Mission orbital parameters summary. . . . . . . . . . . . . . . . 64

4.1 Spectral density of accelerometer errors for satellite axes. . . . . . . . . 71

4.2 Summary of gravity mission configurations. . . . . . . . . . . . . 77

5.1 RMS of errors for South America basin mass estimates for different mascon constraint scenarios shown in Figure 5.14 . . . . . . . . . . . . . 97

5.2 RMS of errors for Greenland basin mass estimates for different mascon constraint scenarios shown in Figure 5.15. . . . . . . . . . . . . . . . 99

5.3 Mean of monthly RMS of 25 South America spatial plots for instrument noise only case. . . . . . . . . . . . . . . . . . . . 112

5.4 RMS of errors for South America basin mass estimates for different mission configurations shown in Figure 5.26: instrument errors only. . . . . 114

5.5 Best fit trend, annual amplitude, and phase values for South America basins: instrument noise only. . . . . . . . . . . . . . . . . . 115 
5.6 Mean of monthly RMS of 25 Greenland spatial plots for instrument noise only case. . . . . . . . . . . . . . . . . 116

5.7 RMS of errors for Greenland basin mass estimates for different mission configurations shown in Figure 5.30: instrument noise only. . . . . . . . 120

5.8 Best fit trend, annual amplitude, and phase values for Greenland basins: instrument noise only. . . . . . . . . . . . . . . . . . 121

5.9 Sampled box sizes and surface areas. . . . . . . . . . . . 125

5.10 Mean of monthly RMS of 25 South America spatial plots for all errors case.136

5.11 RMS of errors for South America basin mass estimates for different mission configurations shown in Figure 5.45: all error sources. . . . . . . . . 138

5.12 Best fit trend, annual amplitude, and phase values for South America basins: all error sources. . . . . . . . . . . . . . . . . . . 139

5.13 Mean of monthly RMS of 25 Greenland spatial plots for all errors case. . 140

5.14 RMS of errors for Greenland basin mass estimates for different mission configurations shown in Figure 5.49: all error sources. . . . . . . . . . . 143

5.15 Best fit trend, annual amplitude, and phase values for Greenland basins: all error sources. . . . . . . . . . . . . . . . . . . . . . . 144

5.16 RMS of temporal aliasing signal over South America. . . . . . . . . . . 149

5.17 RMS of temporal aliasing signal over Greenland. . . . . . . . . . . . . 154

5.18 RMS of errors for South America basin mass estimates for averaging kernel and mascons: GRACE mission with all errors included. . . . . . . 161

5.19 RMS of errors for Greenland basin mass estimates for averaging kernel and mascons: GRACE mission with all errors included. . . . . . . . 163 


\section{Figures}

\section{Figure}

2.1 Geometry of Earth-fixed frame used in study. . . . . . . . . . . . . 22

2.2 Definition of the geoid height $N \ldots \ldots \ldots \ldots$

2.3 Examples of two dimensional spectral densities. Left column: spatial maps with different two dimensional cosine functions, Right column: corresponding two dimensional spectral density. . . . . . . . . . . . . 37

3.1 AOD signal mass variations for 2003. . . . . . . . . . . . 43

3.2 AOD errors for Amazon basin and Greenland mass variations for 2003. 44

3.3 Monthly means of South America and Greenland AOD model error (ECMWF/OMCT minus NCEP/MOG2D). The twelve month mean has been removed. 45

3.4 Truth signal for South America over 24-month simulation (Jan, 2003 to

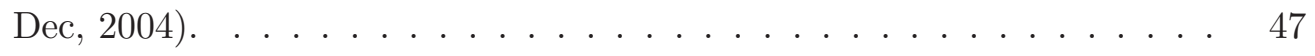

3.5 Map of truth mass trends for constructed Greenland model. . . . . . . 48

3.6 Blue section: elevations below $2000 \mathrm{~m}$ are assigned an annual amplitude of 300 GT. Red section: elevations greater than $2000 \mathrm{~m}$ are assigned and annual amplitude of $50 \mathrm{GT} . \ldots \ldots \ldots$

3.7 Truth signal for Greenland over 24-month simulation (Jan, 2003 to Dec,

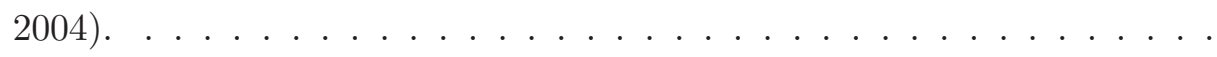


3.8 Numerical simulation procedure flow chart. . . . . . . . . . . . 55

3.9 Groundtracks over South America and Greenland for the two different mission orbital parameters considered in this study. The top two rows show groundtracks for the $480 \mathrm{~km}$ altitude orbit and the bottom two rows show the $250 \mathrm{~km}$ altitude orbit. The columns show the groundtracks for (a) 3 days, (b) 10 days, and (c) 30 days. . . . . . . . . . . .

4.1 Spectral densities for GRACE satellite-to-satellite measurement error. The blue line is range and the green line is range-rate. $\ldots . . . . .69$

4.2 Spectral density for interferometric laser ranging system with a spacecraft separation distance of $50 \mathrm{~km} \ldots \ldots \ldots \ldots 70 \ldots \ldots$

4.3 Spectral density of range-rate error due to accelerometer error. . . . . . 72

4.4 Time series of range-rate errors. . . . . . . . . . . . . . . 72

4.5 One-day time series and histogram of satellite position errors. RMS =

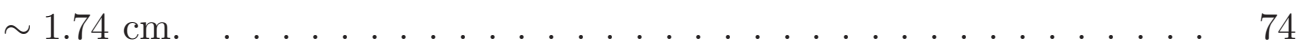

4.6 Illustration of temporal aliasing error. . . . . . . . . . . . . . 75

4.7 Global AOD model error RMS about the mean calculated with twelve monthly means (ECMWF/OMCT minus NCEP/MOG2D). . . . . . 76

4.8 South America and Greenland AOD model error RMS about the mean calculated with twelve monthly means. (ECMWF/OMCT minus NCEP/MOG2D). 77

5.1 Location and size of South America basins. . . . . . . . . . . . . 82

5.2 Location and size of Greenland basins. . . . . . . . . . . . . . . 83

5.3 Spectral density of spatial maps in (a) Figure 5.4 and (b) Figure 5.5. . $\quad 85$

5.4 South America gravity estimates for one constraint matrix. . . . . . . 86 
5.5 Greenland gravity estimates for one constraint matrix. . . . . . . . 87

5.6 Spectral density of spatial maps in (a) Figure 5.7 and (b) Figure 5.8. . $\quad 88$

5.7 South America gravity estimates for separate land and ocean constraints. 89

5.8 Greenland gravity estimates for separate land and ocean constraints. $\quad$ • 90

5.9 Spectral density of spatial maps in (a) Figure 5.10 and (b) Figure 5.11. 91

5.10 South America gravity estimates for uncorrelated basins. . . . . . . . . 92

5.11 Greenland gravity estimates for uncorrelated basins. . . . . . . . . . . 93

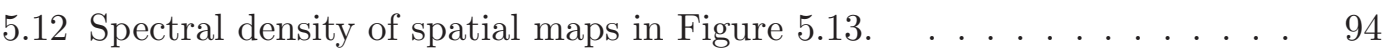

5.13 Greenland gravity estimates for elevation constraint scenario. . . . . . . 95

5.14 South America basin mass variations time series for different mascon constraint scenarios. . . . . . . . . . . . . . . . . 97

5.15 Greenland basin mass variations time series for different mascon constraint scenarios. . . . . . . . . . . . . . . . . . . 98

5.16 South America gravity estimates for GRACE (480 km altitude / K-band / Accelerometer): instrument noise only. . . . . . . . . . . . . . . 102

5.17 South America gravity estimates for GFO Case 1 (480 km altitude / Laser / Accelerometer): instrument noise only. . . . . . . . . . . . . 103

5.18 South America gravity estimates for GFO Case 2 (250 km altitude / K-band / GRS): instrument noise only. . . . . . . . . . . . . . 104

5.19 South America gravity estimates for GFO Case $3(250 \mathrm{~km}$ altitude / Laser / GRS): instrument noise only. . . . . . . . . . . . . . . 105

5.20 Greenland gravity estimates GRACE (480 km altitude / K-band / Accelerometer): instrument noise only. . . . . . . . . . . . 107

5.21 Greenland gravity estimates GFO Case 1 (480 km altitude / Laser / Accelerometer): instrument noise only. . . . . . . . . . . 108 
5.22 Greenland gravity estimates for GFO Case 2 (250 km altitude / K-band / GRS): instrument noise only. . . . . . . . . . . . . . . . . . 109

5.23 Greenland gravity estimates for GFO Case 3 (250 km altitude / Laser / GRS): instrument noise only. . . . . . . . . . . . . . . . . 110

5.24 RMS errors of South America spatial maps for all mission conifigurations: instrument noise only case. . . . . . . . . . . . . . . .

5.25 Mean spectral density of monthly spatial maps for the Truth signal and GRACE and GFO Case 3 error over South America: instrument noise

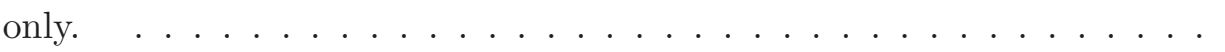

5.26 South America basin mass variations time series for different mission configurations: instrument errors only. . . . . . . . . . .

5.27 Errors in trend and amplitude estimates for each South America basin for GRACE and GFO Case 3: instrument noise only. . . . . . . . . .

5.28 RMS errors of Greenland spatial maps for all mission configurations: instrument noise only case. . . . . . . . . . . . . . . .

5.29 Mean spectral density of monthly spatial maps for the Truth signal and GRACE and GFO Case 3 error over Greenland: instrument noise only.

5.30 Greenland basin mass variations time series for different mission configurations: instrument noise only. . . . . . . . . . . . .

5.31 Total Greenland variation time series for different mission configurations: instrument noise only. . . . . . . . . . . . . . . .

5.32 Errors in trend and amplitude estimates for each Greenland basin for GRACE and GFO Case 3: instrument noise only. . . . . . . . . . 121

5.33 Gravity estimates for $1^{\circ} \times 1^{\circ}$ block of $100 \mathrm{~cm}$ of water for different mission configutations. The location of the Truth signal is shown on each plot. . 
5.34 Mass recovered within a given size block for different mission configurations: instrument noise only. . . . . . . . . . . . . . . . . 125

5.35 South America gravity estimates for GRACE (480 km altitude / K-band / Accelerometer): all error sources. . . . . . . . . . . . . . . . . . . 127

5.36 South America gravity estimates for GFO Case 1 (480 km altitude / Laser / Accelerometer): all error sources. . . . . . . . . . . . . . . . . 128

5.37 South America gravity estimates for GFO Case 2 (250 km altitude / K-band / GRS): all error sources. . . . . . . . . . . . . . . . .

5.38 South America gravity estimates for GFO Case $3(250 \mathrm{~km}$ altitude / Laser / GRS): all error sources. . . . . . . . . . . . . . . . . . 130

5.39 Greenland gravity estimates for GRACE (480 km altitude / K-band / Accelerometer): all error sources. . . . . . . . . . . . . . . . 132

5.40 Greenland gravity estimates for GFO Case 1 (480 km altitude / Laser / Accelerometer): all error sources. . . . . . . . . . . . . .

5.41 Greenland gravity estimates for GFO Case 2 (250 km altitude / K-band / GRS): all error sources. . . . . . . . . . . . . . . . . . . . 134

5.42 Greenland gravity estimates for GFO Case 3 (250 km altitude / Laser / GRS): all error sources. . . . . . . . . . . . . . . .

5.43 RMS errors of South America spatial maps for all mission configurations: all error sources. . . . . . . . . . . . . . . . . . .

5.44 Mean spectral density of monthly spatial maps for the Truth signal and GRACE and GFO Case 3 error over South America: all error sources.

5.45 South America basin mass variations time series for different mission configurations: all error sources. . . . . . . . . . . . . . . . 138 
5.46 Errors in trend and amplitude estimates for each South America basin for GRACE and GFO Case 3: all error sources. . . . . . . . . . . . . 139

5.47 RMS errors of Greenland spatial maps for all mission configurations: all error sources. . . . . . . . . . . . . . . . . . . . . . 140

5.48 Mean spectral density of monthly spatial maps for the Truth signal and GRACE and GFO Case 3 error over Greenland: all error sources. . . . 141

5.49 Greenland basin mass variations time series for different mission configurations: all error sources. . . . . . . . . . . . . . . . . . .

5.50 Total Greenland variation time series for different mission configurations: all error sources. . . . . . . . . . . . . . . . . . . . . . 143

5.51 Errors in trend and amplitude estimates for each Greenland basin for GRACE and GFO Case 3: all error sources. . . . . . . . . . . . . . . 144

5.52 South America temporal aliasing error for the $480 \mathrm{~km}$ orbit. . . . . . . 147

5.53 South America temporal aliasing error for the $250 \mathrm{~km}$ orbit. . . . . . . 148

5.54 Mass variations for South America basins from temporal aliasing. . . . 149

5.55 Greenland temporal aliasing error for the $480 \mathrm{~km}$ orbit. . . . . . . . . . 151

5.56 Greenland temporal aliasing error for the $250 \mathrm{~km}$ orbit. . . . . . . . . . 152

5.57 Mass variations for Greenland basins from temporal aliasing. . . . . . . 153

5.58 Separation of aliasing components for simulations over South America. . 155

5.59 Separation of aliasing components for simulations over Greenland. . . 156

5.60 Total errors and aliasing error components for South America simulations.158

5.61 South America basin mass variations for averaging kernel and mascon estimation methods. . . . . . . . . . . . . . . . . . . 161

5.62 Greenland basin mass variations for averaging kernel and mascon estimation methods. . . . . . . . . . . . . . . . . . . . 162 
5.63 Total Greenland mass variations for averaging kernel and mascon estimation methods. . . . . . . . . . . . . . . 163

A.1 Examples of harmonic types: (i)Zonal (ii)Sectorial (iii)Tesseral. . . . . 177

D.1 Local coordinates. . . . . . . . . . . . . . . . . 190 


\section{Chapter 1}

\section{Introduction}

\subsection{Background and Motivation}

Temporal variations in the gravitational field are the result of a redistribution of mass in the Earth system. Accurate determination of Earth's time-varying gravitational field is thus beneficial to many areas of scientific research. These areas include:

Hydrology - monitoring of global and local water mass variations in the atmosphere and on the continents

Oceanography - determination of sea level change, ocean bottom pressure, ocean heat storage, and ocean tides

Glaciology - monitoring of glacial and polar ice

Solid Earth Science - resolution of tectonic features and estimation of glacial isostatic adjustment

Geodesy - improved accuracy of geoid and improved reference frame for determination of position coordinates

Orbit Determination - improved navigation and orbit determination for low earth orbiting satellites

Orbiting satellites are perturbed by the irregularities in the Earth's gravity field. The Gravity Recovery and Climate Experiment (GRACE) satellite mission determines the shape of Earth's gravity field by accurately measuring these perturbations. This dedicated gravity recovery mission has been successful in determining the Earth's gravity 
field to a much improved level of accuracy at monthly intervals than was possible prior to the launch of GRACE.

Prior to the GRACE mission the Earth's gravity field was determined using decades of satellite tracking data. With this method, it is not possible to determine the gravity field with both high spatial and temporal resolution [Seeber, 2003]. Studies showed that satellite tracking data could be used to estimate temporal variations in the largest spherical harmonic terms. One study by Nerem et al. [2003], estimates monthly values of the $J_{2}$ and $J_{3}$ coefficients with a decade of LAGEOS satellite laser ranging data, and calculates the secular and seasonal variations of these terms. Several efforts have been put forth in which satellite tracking data was optimally combined with surface gravity measurements and altimetry data to solve for the gravity field to a higher spatial resolution than is possible with only satellite data. These efforts sought to accurately determine the mean gravity field and some seasonal variations. Due to insufficient data, the temporal variations of the gravity field, which take place over shorter time intervals, could not be measured.

The GRACE mission was designed to accurately determine the gravitational field of the Earth in 30-day increments with a spatial resolution of $400 \mathrm{~km}$ to 40,000 km. The mission, which launched in March 2002, consists of two identical satellites orbiting at $\sim 480 \mathrm{~km}$ altitude at an inclination of $\sim 89^{\circ}$. The two satellites are separated by $\sim 220 \mathrm{~km}$ in the along-track direction. The K-band microwave ranging system accurately provides the dual range and range-rate measurements between the satellites while the Global Positioning System (GPS) receivers on each satellite allow for precise orbit determination and time-tagging of the satellite-to-satellite range measurements. Each GRACE satellite also carries a star tracker for attitude determination and a high precision accelerometer which measures the sum of all non-conservative forces [Tapley 
et al., 2004b].

As described in the next section, the results from the GRACE mission have allowed tremendous achievements in a variety of scientific disciplines. It is expected, however, that a follow-on gravity recovery mission to GRACE, with some improvements, could measure variations in the gravity field to an even greater spatial resolution, thus furthering the scientific objectives of these missions. The primary focus of this work is to investigate the improvements and benefits which would be made possible from a follow-on gravity recovery mission to GRACE.

\subsection{GRACE Results}

Beginning in April 2002, GRACE data has been used to estimate the Earth's gravity field at monthly intervals. The released monthly gravity estimates are a set of spherical harmonic coefficients to degree and order 120. These monthly solutions contain valuable information of temporal variations in the gravity field which can be used to study various geophysical phenomena. Prior to their release, several well-known geophysical processes which cause temporal variations in the gravity field are modeled and removed from the GRACE monthly estimates. The removed processes include the solid Earth and oceanic tides, the pole-tide effects, atmospheric pressure, and non-tidal oceanic mass variations [Tapley et al., 2004b]. The monthly gravity estimates from GRACE, therefore, contain information of the unmodeled geophysical processes, which consist of changes in continental water storage, and residual signal from the atmosphere, ocean, and tides (due to imperfect modeling).

Given a set of spherical harmonics, the gravity field can be plotted as geoid height or as equivalent water height (see Section 2.3). Several methods have been developed to smooth the data in order to provide a more coherent picture of mass 
variation. These methods are motivated by the fact that the accuracy of the gravity field solutions degrade as the spatial scale decreases. A commonly used approach is a Gaussian averaging kernel, which was developed by Jekeli [1981], and is summarized by Wahr et al. [1998]. For this technique the averaging radius is varied to observe changes in the gravitational signal at different spatial scales. Localized averaging kernels can be used to extract the mass variations in specific regional areas (see Section 2.5) [Swenson and Wahr, 2002].

Several studies have been performed to assess the accuracy of the gravity field estimates provided by the GRACE mission. The accuracy of the estimated gravity field is affected by the spherical harmonic truncation degree or the selected smoothing radius [Wahr et al., 2006, 2004]. Wahr et al. [2004] report water equivalent height accuracies of $0.7 \mathrm{~cm}$ for a $1500 \mathrm{~km}$ smoothing radius, $1.0 \mathrm{~cm}$ for a $1000 \mathrm{~km}$ radius, and $1.4 \mathrm{~cm}$ for a $750 \mathrm{~km}$ radius for the annual signal. It is shown by Wahr et al. [2006] that the monthly GRACE errors are also dependent on latitude. A global map of $1 \sigma$ uncertainties for the August 2003 GRACE field shows that the uncertainties vary from $0.8 \mathrm{~cm}$ at high latitudes to $2.8 \mathrm{~cm}$ at lower latitudes. This latitude dependence is due to the ground coverage of the GRACE satellites.

In short, the knowledge of the time-varying gravitational signal as provided by GRACE has enabled tremendous improvements in the understanding of the Earth system. A brief summary of scientific uses for GRACE data is now given.

\subsubsection{Hydrology}

The hydrologic cycle is essential for life on Earth and knowledge of continental water balance could have important socioeconomic impacts. On land, water is stored in lakes, rivers, aquifers, or as snow, ice, soil moisture or vegetative water. Prior to the 
launch of GRACE the temporal and spatial variations of terrestrial water at global and regional scales were poorly known [Rodell and Famiglietti, 1999]. The determination of water changes over land was limited by ground-based techniques, which only provide point estimates of water storage. Extensive modeling is then required to generate global estimates of land water storage from the in situ hydrological measurements. With the monthly GRACE gravity fields, global maps of continental water storage are available and used to gain a new understanding of the components which make up the water balance equation:

$$
\frac{\partial S}{\partial t}=P-E T-R
$$

where $S$ is land water storage, $P$ is precipitation, $E T$ is evapotranspiration, $R$ is runoff, and $\partial t$ is the change in time. GRACE data can be used to directly estimate the land water storage component. Estimates of the other components of Eq. (1.1) can be found by combining the GRACE estimates of land water storage with estimates of the other variables which are found using other techniques. Several examples of the hydrologic applications of GRACE are given below.

Some of the earliest results of mass variability as measured by GRACE are presented by Tapley et al. [2004a]. GRACE data are used to compute global maps of the sine (spring-fall) and cosine (winter-summer) annual variations in the gravity field and are compared to the Global Land Data Assimilation System (GLDAS) [Rodell et al., 2004b] hydrology model. The shape of the maps agree well but the GRACE estimates have larger amplitudes. The authors suggest that the disagreement is likely caused by the fact that GLDAS did not model deep groundwater, snow persistence, or variations on Antarctica. More recently, Syed et al. [2008] shows good agreement between global estimates of terrestrial water storage change from GRACE and GLDAS with a global 
Root Mean Square (RMS) error of $\sim 1 \mathrm{~cm} /$ month. Similarly, Swenson and Milly [2006] use GRACE data to form global estimates of the amplitude and phase of the seasonal land water storage signal and compare the results to land water storage estimates from five different hydrology models. Though the global maps appear to be similar in shape, the results at regional scales differ noticeably in amplitude and phase. The authors conclude that the GRACE results reveal biases in the hydrology models at low latitudes and hypothesize that the hydrology models poorly estimate the precipitation and river water storage amounts.

GRACE data has been used by many researchers to obtain new estimates of regional changes in terrestrial water or to validate local estimation techniques for a number of river basins in the United States and around the world. Swenson et al. [2006] show that good agreement exists between terrestrial water storage estimates from GRACE and in situ hydrological observations from a network of observing sites in Illinois. Similarly, Strassberg et al. [2007] show that GRACE water storage estimates agree well with the sum of groundwater storage measurements and simulated soil moisture for the High Plains Aquifer in the United States. Swenson et al. [2008b] combine GRACE data with soil moisture data to generate estimates of variations in groundwater storage for the region observed by the Oklahoma Mesonet. Crowley et al. [2006] use GRACE data to form terrestrial water storage estimates for the Congo Basin in Africa, showing a sizable seasonal signal and a long-term trend of mass loss. Niu et al. [2007] use GRACE data to provide estimates of snow mass variations for large Arctic regions.

As previously described, GRACE data, which directly measures mass variation, can be combined with models or other estimation techniques to provide estimates of other variables in the water balance equation. Swenson and Wahr [2006a] combine GRACE estimates of terrestrial water storage with basin discharge data to form regional 
estimates of $P-E T$. The $P-E T$ estimates are computed for the Mississippi and the Ohio-Tennessee river basins at monthly and seasonal time scales and show relatively good agreement with hydrological models. The seasonal estimates of $P-E T$ are then combined with precipitation data to form seasonal estimates of evapotranspiration for the river basins. The authors state that the GRACE results validate the hydrological models and that the ability of GRACE to provide new estimates of evapotranspiration is an important contribution to global hydrology. Similarly, evapotranspiration values for the Orinoco, Amazon, Tocantins, Parana, Niger, Congo, Ganges, and Mekong river basins are computed by Ramillien et al. [2005] and evapotranspiration values over the Mississippi river basin are computed by Rodell et al. [2004a]. Syed et al. [2005] combine GRACE estimates of land water storage with precipitable water and vapor flux divergence data from the European Centre for Medium-Range Forecasts (ECMWF) to compute estimates of total basin discharge for the Amazon and Mississippi river basins. Differences exist between the GRACE basin discharge estimates and observed streamflow which can be explained in part by unmonitored changes in groundwater.

\subsubsection{Oceanography}

GRACE has provided a wealth of new information regarding water mass variations in the ocean. This knowledge is of tremendous importance for several reasons. First, variations in sea level is of primary concern for populations currently living near the current sea level. The unique information provided by GRACE allows the scientific community to determine more accurately what sources are contributing to changes in the sea level. Also, when combined with altimetry data, GRACE can provide calculations of ocean heat storage. The large amount of energy stored in the oceans as heat can have tremendous impacts on climate and can act as a source of energy for severe 
storms. A better knowledge of ocean heat storage allows for improved prediction of climate change, long-range weather forecasting, and hurricane strength prediction [Jayne et al., 2003].

There are two components to sea level change. Steric sea level change is caused by variations in the water density as a result of changes in temperature or salinity. The eustatic portion of sea level change, which is measured by GRACE, represents the exchange of water mass between the ocean and other reservoirs. Eustatic sea level change is from water mass changes in glacial and polar ice, terrestrial water, and the atmosphere. Altimeters are sensitive to total sea level change which is the sum of the steric and eustatic components. GRACE data can be combined with altimeter data to differentiate between the two sources of sea level change.

A variable of primary interest in oceanography is the global mean sea level (GMSL) and its changes. Long-term sea level rise is the result of ocean warming, melting of glaciers and polar ice, and glacial isostatic adjustment (GIA) [Chen et al., 2005]. It is expected that GRACE will be very useful in separating the steric and eustatic portions of interannual sea level change, but a longer time series of data will be required before any valid conclusions can be made [Cazenave and Nerem, 2004]. Sea level changes which occur at time periods of less than a few days are the result of tides, evaporation, and precipitation and for the most part are not recovered by GRACE due to limitations in the temporal sampling. GRACE data has proved useful for estimating seasonal variations in GMSL.

In the work of Chambers et al. [2004], the eustatic estimates of the GMSL from GRACE are compared to the estimates calculated from the TOPEX/Poseidon (T/P) and Jason-1 altimeter data corrected for steric variations. The results show excellent agreement, with a difference in the amplitude of the signal of $0.1 \mathrm{~mm}$ and a phase dif- 
ference of only 12 degrees. The authors state that this work validates that GRACE is recovering mass variations in the ocean and suggest that the disagreements could be real interannual variations measured by GRACE. Chen at al. [2005] compute variations in the eustatic component of GMSL with GRACE data, the T/P and Jason-1 altimeter data corrected for steric variations, and GLDAS combined with National Center for Environmental Prediction (NCEP) estimates of water vapor. The results also show very good agreement between the independent methods of estimating the eustatic GMSL. Chambers et al. 2006a use GRACE data to form monthly maps of the eustatic component of sea level and shows good spatial agreement with steric-corrected altimetry estimates.

Chambers [2006b] combines GRACE and Jason-1 altimeter data to form estimates of the steric component of sea level variation. The resulting estimates of steric sea level (SSL) are compared to the World Ocean Atlas 2001 (WOA01) model and show that with the use of GRACE and altimetry data, the estimates of the seasonal SSL signal improves for $\sim 75 \%$ of the ocean. Estimates of SSL, when combined with salinity data, can be used to form accurate estimates of ocean heat content [Jayne et al., 2003].

GRACE data has also proved useful in estimating ocean bottom pressure variations, which are the sum of oceanic and atmospheric variations. Ponte et al. [2007] estimate the seasonal cycle of ocean bottom pressure with GRACE and suggest that constraining the ECCO (Estimating the Circulation and Climate of the Ocean) general circulation model with information from GRACE could improve the model. Bingham and Hughes [2006] use GRACE data to compute seasonal variations in ocean bottom pressure in the North Pacific.

For the majority of GRACE applications, the ocean, Earth, and atmospheric tides are considered a source of error for the gravity estimates, as these signals are removed 
prior to the release of the monthly solutions. However, a few studies have shown that GRACE data can be used to improve tide models or to verify improvements in the tide models. In the work of Han et al. [2005] GRACE data are used to estimate the $M_{2}$ and $S_{2}$ tides underneath the Filchner-Ronne and Larsen ice shelves in Antarctica. The results agree well with the FES2004 (Finite Element Solution) and CATS02.01 (Circum-Antarctic Tidal Simulation) tide models and the authors conclude that GRACE can provide useful observations of ocean tides. This is an important contribution to oceanography as the considered area has few tide gauges. More recently, Han et al. [2007] use GRACE data to estimate the $M_{2}, S_{2}$, and, $O_{1}$ ocean tides in the Antarctic showing good qualitative agreement to sparse in situ tide measurements. Desai and Yuan [2006] argue that improvements in tide models will result in reduced residuals for the K-band range-rate measurements, concluding that GRACE data can be used to determine the relative accuracies between tide models.

A final benefit of GRACE on oceanography is the improved mean dynamic ocean topography (MDT) estimates which have resulted from a much improved knowledge of the static geoid. In addition to providing monthly estimates of the gravity field, GRACE data has also been used to form much improved estimates of the static geoid than was previously possible. Improved solutions of the static gravity field have been determined using GRACE data alone, or in some cases as a combination of the GRACE data, altimetry data, and ground-based gravity measurements. A more accurate static geoid allows for more accurate calculation of the MDT, which is defined as the mean height of the sea surface above the geoid. These variations from the geoid are caused by wind and buoyancy forcing [Bingham and Haines, 2006]. Therefore, knowledge of the MDT can be used to study ocean circulation which is an integral part of climate studies. The Gravity Field and Steady-State Ocean Circulation Explorer (GOCE) mis- 
sion, which was launched in March 2009, will measure the static geoid more accurately than GRACE [Bingham and Haines, 2006] and thus further improve the accuracy of the MDT estimates. Results describing the improved estimation of the MDT using GRACE data are presented by [Bingham and Haines, 2006; Birol et al., 2005; Castruccio et al., 2006; Jayne, 2006; Tapley et al., 2003].

\subsubsection{Glaciology}

GRACE has been used to monitor changes in glacial and polar ice, which is a significant part of the global water mass balance equation described by Eq. (1.1). So far, the majority of studies have focused on measuring mass changes over Greenland and Antarctica, since the gravity signals are large, and variations in the ice mass balance of these ice sheets will result in important changes in the global mean sea level. GRACE presents a unique ability to regularly measure these changes at large spatial scales and to determine their effect on sea level change. Other techniques of measuring ice mass variations in Greenland and Antarctica include airborne laser altimetry measurements and satellite radar interferometry [Ramillien et al., 2006]. Large uncertainties exist with these techniques due to insufficient spatial and temporal coverage and their inability to distinguish between ice mass loss and changes in snowpack [Chen et al., 2006b].

The GRACE estimates of secular changes in ice mass balance over Greenland and Antarctica are contaminated by the gravity signal from glacial isostatic adjustment, which is the viscoelastic response of the solid Earth to the melting of glaciers over the past few thousand years. It is not possible to distinguish the difference between secular ice mass changes and GIA using GRACE data alone [Velicogna and Wahr, 2005]. However, changes in the rate of mass loss are measurable since the rate of mass change caused by GIA is constant [Chen et al., 2006b]. Further discussion of GIA is 
presented in Section 1.2.4.

Velicogna et al. [2005] calculate the seasonal signal for Greenland with more than two years of GRACE data. Their estimates compare well to mass change estimates calculated from ERA40 (40-year ECMWF Re-Analysis), concluding that the differences could be real signal recovered by GRACE. The authors suggest that this signal consists of ice discharge, blowing-snow sublimation, and delayed runoff as well as errors in the ERA40 model. Luthcke et al. [2006] also observe a strong seasonal signal in the Greenland ice mass. Their work shows that the drainage systems with an elevation below $2000 \mathrm{~m}$ have a strong seasonal signal while the higher elevation areas do not.

Of primary importance are the interannual variations in the Greenland ice mass balance, as this is an indicator of climate change and will likely be a major contributor to long-term changes in global mean sea level. A number of research groups have calculated the rate of ice mass loss for Greenland using a variety of methods and different time periods of the GRACE data [Chen et al., 2006b; Luthcke et al., 2006; Ramillien et al., 2006; Velicogna and Wahr, 2006a; Wouters et al., 2008]. While their calculations of the rate of mass loss differ, all authors do agree that GRACE results conclusively show a sizable amount of ice mass loss over the time period of the mission. Velicogna et al. [2006a] and Chen et al. [2006b] both show an acceleration in the Greenland mass loss in 2004. Wouters et al. [2008] also observe an acceleration in mass loss over the time period February 2003 to January 2008. Luthcke et al. [2006] show that areas above 2000 $m$ have had an increase in ice mass, while the lower elevations experienced large mass losses over recent years. According to [Chen et al., 2006b], melting rates as calculated by GRACE data show good agreement when compared to estimates from satellite radar interferometry data.

Several investigations have used GRACE data to calculate estimates of interan- 
nual variations in the Antarctic ice mass [Chen et al., 2006a; Ramillien et al., 2006; Velicogna and Wahr, 2006b]. Both Chen et al. [2006a] and Ramillien et al. [2006] observe mass losses in Western Antarctica and an accumulation of mass in Eastern Antartica with Ramillien et al. [2006] claiming good agreement with mass variations calculated using satellite radar altimetery. Velicogna and Wahr [2006b] calculate much larger mass losses in Western Antartica than the other reports and estimate a mass balance in the Eastern portion. These discrepancies are likely caused by large errors in the GIA models [Ramillien et al., 2006].

Chen et al. [2007] use GRACE data to show that the Patagonia Icefield of South America experienced large ice mass losses over the time period April 2002 to December 2006. The determined rate of ice mass loss agrees well with previously determined values from topographic and cartographic data.

Using a local mascon technique Luthcke et al. [2008] estimate mass changes in the Gulf of Alaska from April 2003 to September 2007 with a 10-day sampling. The results show a strong annual signal as well as noticeable mass losses which agree well with air-borne laser-altimeter and Shuttle Radar Topography Mission (SRTM) studies. The mascon solutions clearly show the affects of the record high temperatures of summer 2004 and the large snowfall of winter 2007.

\subsubsection{Solid Earth Science}

GRACE data has proved useful for a variety of solid earth science applications, including the estimation of glacial isostatic adjustment, determination of crustal displacements, and the measurement of coseismic and postseismic deformation caused by the 2004 Sumatra-Andaman earthquake.

Glacial isostatic adjustment is the response of the Earth to the large-scale deglacia- 
tion which ended about 10,000 years ago and the redistribution of solid earth mass due to GIA is contained in the GRACE data. Measurements of GIA can be used to infer the viscosities of the upper and lower mantle as well as the lithospheric thickness. Simulations suggest that GRACE mission data will provide improved estimates of these parameters [Velicogna and Wahr, 2002]. Chen et al. [2006b] state that the GRACE data set will need to be extended until 2010 in order to effectively separate the ice mass change from the GIA signal. At that point, improved estimates of glacial mass change and the mentioned solid Earth parameters should be realized. A recent study by Barletta et al. [2009] does use GRACE data to estimate GIA by removing the known present-day linear mass change signals from the GRACE gravity estimates. Values of the upper and lower mantle viscosities are estimated as a part of the study.

In the work of van Dam et al. [2007] predictions of vertical crustal displacements over Europe as calculated by GRACE are compared to measurements obtained using a network of GPS sites. The authors conclude that GRACE provides more accurate estimates of annual variations in crustal displacement than is currently possible with GPS. Shin et al. [2009] use the GRACE combined gravity model GGM02C, which is formed with a combination of GRACE, altimeter, and ground gravity measurements, to estimate the three-dimensional Moho fold structure of the Tibetan region.

A very interesting application of GRACE data has been in the study of the great 2004 Sumatra-Andaman earthquake. Using GRACE data, Han et al. [2008] observe the coseismic displacements and the postseismic deformation caused by the massive earthquake. Steady state and transient viscosities of the asthenosphere are estimated from the results. 


\subsubsection{Geodesy}

GRACE has also benefitted the geodetic sciences community with some interesting contributions. Nastula et al. [2007] use the degree-2, order-1 harmonics from the GRACE estimates to compute the polar motion excitation functions $\chi_{1}$ and $\chi_{2}$ and show that the GRACE solutions agree well with polar motion as determined by direct observation and modeling of geophysical fluids. Swenson et al. [2008a] combine GRACE data with ocean model data to form new estimates of variations in the Earth geocenter.

\subsubsection{Errors}

There are two categories of error sources which limit the accuracy of the GRACE gravity estimates. These are instrument measurement errors and modeling errors. Instrument errors include errors in the K-band microwave ranging device, the accelerometers, and the GPS measurements. These error sources affect the gravity estimates at different spatial wavelengths [Kim, 2000]. Modeling errors are caused by imperfections in the geophysical models used to remove unwanted signal from the GRACE gravity estimates. These include errors in the atmospheric models, oceanic models, and the solid Earth and ocean tide models. Any imperfections in these models will appear as a gravitational signal in the estimates. Studies have also shown that temporal aliasing is a major contributor to the GRACE errors [Han et al., 2004; Ray and Luthcke, 2006; Thompson et al., 2004]. Aliasing errors are the result of short-period variations in the Earth's gravity field which alias into the monthly mean estimates due to limitations in the temporal sampling of the GRACE satellites. These short-period variations are present in the hydrological, atmospheric, tidal, and non-tidal oceanographic signals.

The main thrust of this work is an investigation into how a reduction in the 
instrument errors, specifically the ranging measurement errors and the error associated with the on-board accelerometers, would improve the gravity estimates from a GRACElike mission. It is important to note that scientists are constantly working to improve the geophysical models discussed above. Much work has also been done to develop methods which minimize the affects of these error sources on the GRACE gravity estimates. As this work progresses, it will only enhance the ability of GRACE, and a GRACE Follow-On mission to more accurately detect the variations in the gravitational field.

\subsection{Previous Investigation into a GRACE Follow-On Mission}

A GRACE Follow-On (GFO) mission would have tremendous benefits for the scientific community. In addition to extending the time series of data, currently being provided by GRACE, a follow-on mission may be able to determine the Earth's gravity field with an improved spatial and/or temporal resolution. The desire for an improved spatial resolution would likely drive the design of a follow-on mission. Some work has already been done contributing to the design of a potential future satellite gravity recovery mission and an overview is given here.

Some suggestions for a GFO mission were made by Bender et al. [2003]. Of primary interest to this investigation is the possible use of laser heterodyne measurements between the two orbiting spacecraft, as opposed to the K-band microwave ranging system employed by GRACE. Since a laser ranging device could measure the range and range-rate between the spacecraft with better accuracy, a GFO mission equipped with such a device should recover the Earth's gravity field with a greater spatial resolution.

Bender et al. [2003] also discuss the possibility of flying a GFO mission with a drag-free system. This is accomplished by isolating a proof mass within the satellite from the surrounding environment, making it unsusceptible to the non-conservative 
forces acting on the satellite [Seeber, 2003]. A micro-thruster system would then be used to maintain the position of the satellite with respect to the proof mass so that the satellite orbit would not be affected by drag. This would eliminate the need to include the accelerometer data in the data processing which should improve the accuracy of the gravity estimates. A detailed investigation into the feasibility of a drag-free follow-on mission is performed by Marchetti et al. [2008]. The work shows that assuming $87 \mathrm{~kg}$ of propellant mass, a GRACE follow-on mission would have a lifetime of 0.76 years for an orbital altitude of $160 \mathrm{~km}$ and 2.27 years for an altitude of $225 \mathrm{~km}$. To extend the $225 \mathrm{~km}$ altitude case to 5 years would require approximately $170 \mathrm{~kg}$.

A GRACE follow-on mission consisting of a four satellite cartwheel formation was first proposed by Bender et al. [2003]. In a study by Wiese et al. [2008], four different GRACE follow-on satellite configurations are considered: a two-satellite collinear pair similar to GRACE, a four-satellite configuration with two collinear pairs, a two-satellite cartwheel formation, and a four-satellite cartwheel formation. The results show that for a case where only the satellite-to-satellite ranging noise is considered, the cartwheel cases perform about an order of magnitude better than the collinear cases and drastically reduce the striping in the spatial error maps. When errors due to the mismodelling of the time-variable signal in the atmosphere and oceans is included, the cartwheel orbits actually perform worse than the collinear cases at high spherical harmonic degrees.

It is of course advantageous to fly a follow-on mission in as low an altitude as possible since the gravity signal attenuates with distance. The limitation to the altitude is placed by the non-conservative forces which are greater at lower altitudes and the amount of thrusting required to maintain the desired orbit of the spacecraft in the presence of these forces.

This work is in conjunction with the development of an interferometric laser 
ranging system by Ball Aerospace \& Technologies Corp., Boulder, CO. This project, which was completed in December 2006, resulted in the successful development of a laser ranging device for use on a GFO mission. Details regarding this project are presented in [Dehne et al., 2009; Nerem et al., 2006; Pierce et al., 2008]. In summary, the new instrument will improve the range-rate accuracies from $\sim 0.2 \mu / \mathrm{s}$ to $\sim 0.6 \mathrm{~nm} / \mathrm{s}$. It is expected that this substantial improvement could enable a GFO to measure the gravity field to an improved spatial resolution, thus benefiting all previously discussed areas of scientific research.

\subsection{Research Overview}

The goal of this work is to develop a thorough error analysis of a follow-on gravity mission to GRACE (GFO). As described in the previous section, Ball Aerospace \& Technologies Corp., Boulder, CO has developed an interferometric laser ranging system with a future satellite gravity mission in mind. It is expected that a GRACE-like mission equipped with the interferometric laser ranging system would improve the spatial resolution to which the Earth's gravitational field is observed from space. An improved resolution in the estimates would enable further achievements in the areas of scientific study discussed in Section 1.2. Additionally, GFO missions which employs a drag-free system and a reduced orbital altitude are also considered.

In this work, full numerical simulations are performed to determine the expected performance of three different GFO mission configurations and are compared to the simulated performace of the current GRACE mission. These simulations account for all major sources of error which affect the estimation of time-variable gravity. Numerical simulations for the GFO missions utilize the error budget for the interferometric laser ranging system presented in Pierce [2008]. The results of this trade study are useful for 
planning a future gravity mission similar to GRACE.

An important part of this research is the quantification of the temporal aliasing error on the time series of the simulated regional gravity estimates for the different mission configurations. The simulations show that the aliasing errors dominate, so the sources of this error are investigated separately to determine their effects on the solutions.

A final key part of this work is a comparison between the traditional gravity estimation method of spherical harmonics, and a localized mascon gravity estimation technique. This comparison is relevant because current GRACE data are processed using a variety of techniques, and understanding the effectiveness of these two estimation methods at recovering the time-variable gravity signal in the GRACE data may shed light on how the current and future data should be handled.

The work presented is organized into five parts:

(a) Chapter 2 of this study develops the mathematical expressions that are used in defining the gravitational field of the Earth. A global representation of the gravity field using spherical harmonics is presented, followed by a description of the mascon representation, which can be used to describe both a global and a regional gravity field. The averaging kernel technique which recovers regional mass variation from global sets of spherical harmonics is described. The final section of the chapter summarizes the statistical tools which are used to compare the accuracy of the simulated gravity estimates to the true signal.

(b) Chapter 3 describes in detail the numerical simulation procedure used in this study. This chapter includes a description of the force models used in the simulations, and the linear estimation theory that is implemented by the simulation software. The orbital parameters for GRACE and GFO are summarized. 
(c) Chapter 4 contains detailed descriptions of all sources of error included in the numerical simulations. The described error sources are the inter-satellite measurement errors, the accelerometer and drag-free errors, the satellite positioning errors, and the temporal aliasing errors. The final section summarizes the GRACE and the three considered GFO mission configurations, which are defined by the orbital parameters and sources of error of the included instrument suite.

(d) Chapter 5 is a presentation of all results of the numerical simulations. First, basin locations for South American and Greenland are defined. Next, the simulated gravity estimates are presented for different mascon estimation methods to determine the ideal method for the results to be processed and presented. Gravity estimates are then given and analyzed for two cases: 1.) instrument noise only (no atmospheric, oceanic, and tidal model errors), and 2.) all error sources. The various components of the temporal aliasing errors are investigated. Lastly, the results obtained with spherical harmonic averaging kernels, and the mascons method are presented.

(e) Chapter 6 contains the summary and conclusions of the study along with recommendations for a potential future follow-on gravity mission to GRACE. 


\section{Chapter 2}

\section{The Earth Gravitational Field}

\section{$2.1 \quad$ Introduction}

The gravitational field of the Earth is spatially irregular. Anomalies in the gravitational field are due to the non-sphericity of the Earth and the inhomogeneous mass distribution within its body and on its surface. The ellipticity is dominant and a reference ellipsoid is used as a first approximation of the Earth's shape and gravitational field. The geoid is commonly used to describe the gravitational field with respect to the reference ellipsoid and is represented by spherical harmonics. It is also common for the gravity anomalies to be represented in terms of equivalent water height.

\subsection{Definition of the Geoid}

The geoid is defined as the surface within or around the Earth that is everywhere normal to the direction of gravity and coincides with mean sea level in the oceans [editors, 2003]. The geoid is determined by the total potential of gravity $W(r, \phi, \lambda)$, which is the combination of gravitational potential $V(r, \phi, \lambda)$ and the rotational potential. It is described by

$$
W(r, \phi, \lambda)=V(r, \phi, \lambda)+\frac{1}{2} \omega^{2} r^{2} \cos ^{2} \phi
$$


where $r$ is the radial distance from the center of the Earth, $\phi$ is the geocentric latitude, $\lambda$ is the longitude, and $\omega$ is the rotation rate of the Earth. Any surface for which $W(r, \phi, \lambda)=$ constant is called an equipotential surface. The geoid is the equipotential surface that best approximates with mean sea level.

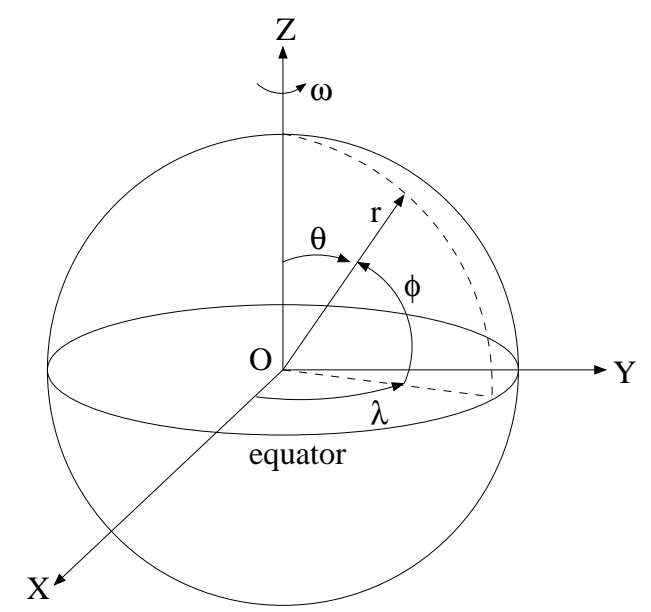

Figure 2.1: Geometry of Earth-fixed frame used in study.

The shape of the earth is often approximated as a reference ellipsoid and can be defined by the flattening,

$$
f=\frac{a-b}{a}
$$

where $a$ and $b$ are the equatorial and polar radius respectively. Currently $f=1 / 298.257$ is the adopted best value for the Earth [Seeber, 2003]. The equatorial radius of the Earth differs from the polar radius by $\sim 21 \mathrm{~km}$. In Section 2.3 the reference ellipsoid is defined using spherical harmonics.

Figure 2.2 shows both the reference ellipsoid and the geoid. The difference, $N$, of the geoid from the reference ellipsoid is called the geoid height. The expression for the geoid height is given in Section 2.3. 


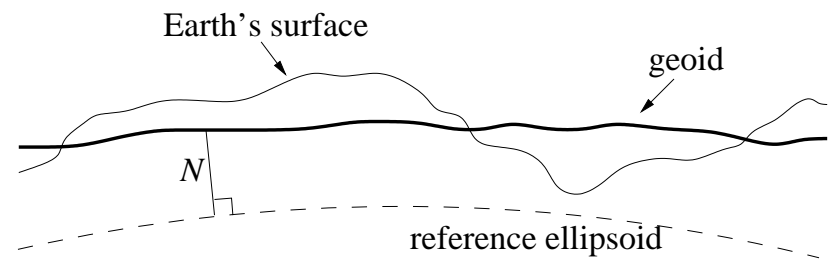

Figure 2.2: Definition of the geoid height $N$

\subsection{Spherical Harmonics}

The gravitational potential and the geoid are conventionally represented using spherical harmonics. The mathematical formulation used to describe the geoid is given here. For a more detailed derivation of the material presented in this section refer to [Kaula, 2000; Seeber, 2003; Torge, 2001]. Further definitions are also given in Appendix A.

For a point outside of the Earth, i.e. an orbiting spacecraft, the gravitational potential satisfies Laplace's equation,

$$
\nabla^{2} V=\frac{\partial^{2} V}{\partial x^{2}}+\frac{\partial^{2} V}{\partial y^{2}}+\frac{\partial^{2} V}{\partial z^{2}}=0
$$

which is given here in rectangular coordinates.

Since the Earth is relatively spherical in shape, it is useful to use spherical coordinates which are defined as,

$$
\begin{aligned}
& x=r \cos \phi \cos \lambda, \\
& y=r \cos \phi \sin \lambda, \\
& z=r \sin \phi .
\end{aligned}
$$

The objective is to convert Laplace's equation to spherical coordinates. The partial derivatives of the spherical coordinates with respect to the rectangular coordinates are determined by differentiating Eq. (2.4) with respect to $r, \phi$, and $\lambda$ and solving for $d r$, 
$d \phi$, and $d \lambda$. The resulting partial derivatives are used to solve for Laplace's equation in spherical coordinates, and is given by,

$$
\nabla^{2} V=\frac{1}{r^{2}} \frac{\partial}{\partial r}\left(r^{2} \frac{\partial V}{\partial r}\right)+\frac{1}{r^{2} \cos \phi} \frac{\partial}{\partial \phi}\left(\cos \phi \frac{\partial V}{\partial \phi}\right)+\frac{1}{r^{2} \cos ^{2} \phi} \frac{\partial^{2} V}{\partial \lambda^{2}}=0
$$

The solution to Eq. (2.5) is derived by Kaula [2000] using separation of variables. The resulting expression for the gravitational potential is,

$$
V=\frac{\mu}{r}\left(1+\sum_{n=1}^{\infty} \sum_{m=0}^{n}\left(\frac{R_{\oplus}}{r}\right)^{n} P_{n m}(\sin \phi)\left(C_{n m} \cos m \lambda+S_{n m} \sin m \lambda\right)\right)
$$

where $C_{n m}, S_{n m}$ are mass integrals called geopotential coefficients, $P_{n m}$ are the associated Legendre functions, $R_{\oplus}$ is the radius of the Earth and $\mu=G M$ where $G$ is the universal gravitational parameter and $M$ is the mass of the Earth. The subscripts $n$ and $m$ refer to the degree and order respectively.

It is conventional to use the fully normalized geopotential coefficients and the fully normalized associated Legendre functions. These are defined as,

$$
\begin{aligned}
& \tilde{P}_{n m}(\sin \phi)=\left[\frac{k(2 n+1)(n-m) !}{(n+m) !}\right]^{1 / 2} P_{n m}(\sin \phi), \\
& \left\{\begin{array}{c}
\bar{C}_{n m} \\
\bar{S}_{n m}
\end{array}\right\}=\left[\frac{(n+m) !}{k(2 n+1)(n-m) !}\right]^{1 / 2}\left\{\begin{array}{c}
C_{n m} \\
S_{n m}
\end{array}\right\},
\end{aligned}
$$

with $k=1$ for $m=0$, and $k=2$ for $m \neq 0$ [Seeber, 2003]. Substituting the normalized definitions into Eq. (2.6) the expression for the gravitational potential becomes,

$$
V=\frac{\mu}{r}\left(1+\sum_{n=1}^{\infty} \sum_{m=0}^{n}\left(\frac{R_{\oplus}}{r}\right)^{n} \tilde{P}_{n m}(\sin \phi)\left(\bar{C}_{n m} \cos m \lambda+\bar{S}_{n m} \sin m \lambda\right)\right) .
$$

The disturbing potential is defined by the difference of the actual gravitational potential $W$ and the normal gravity $U$, which is defined by the reference ellipsoid. At the point $P$ the disturbing potential is defined by,

$$
T_{P}=W_{P}-U_{P}
$$


The elements $W$ and $U$ both have a gravitational and centrifugal part as defined in Eq. (2.1). Since the rotation rate of the Earth is well known the centrifugal parts of $W$ and $U$ are assumed to be the same. The disturbing potential also satisfies Laplace's equation and a solution is given by,

$$
T=\frac{\mu}{r} \sum_{n=2}^{\infty} \sum_{m=0}^{n}\left(\frac{R_{\oplus}}{r}\right)^{n} \tilde{P}_{n m}(\sin \phi)\left(\bar{C}_{n m}^{*} \cos m \lambda+\bar{S}_{n m}^{*} \sin m \lambda\right) .
$$

with

$$
\begin{aligned}
& \bar{C}_{n m}^{*}=\text { observed } \bar{C}_{n m} \text { - reference } \bar{C}_{n m}, \\
& \bar{S}_{n m}^{*}=\text { observed } \bar{S}_{n m} \text { - reference } \bar{S}_{n m} .
\end{aligned}
$$

Here the reference ellipsoid is defined in terms of spherical harmonics. The reference ellipsoid is often defined by $\bar{C}_{20}$ and $\bar{C}_{40}$ [Seeber, 2003], but other even zonal terms $(m=0)$ can be included. In Eq. (2.11) the assumption has been made that the origin of the coordinate system has been placed at the center of mass of the Earth. For this case, the disturbing potential becomes zero for $n=1, m=0,1$ and the summation begins at $n=2[$ Seeber, 2003].

The expression for the geoid height is found by dividing the disturbing potential $T$ by normal gravity $\gamma[$ Torge, 2001]. Thus,

$$
N=\frac{T}{\gamma}=\left(\frac{\mu}{r \gamma}\right) \sum_{n=2}^{\infty} \sum_{m=0}^{n}\left(\frac{R_{\oplus}}{r}\right)^{n} \tilde{P}_{n m}(\sin \phi)\left(\bar{C}_{n m}^{*} \cos m \lambda+\bar{S}_{n m}^{*} \sin m \lambda\right) .
$$

In practice it is necessary to truncate the infinite summation at some maximum degree $\left(N_{\max }\right)$ to which the gravitational potential can be determined. Also a spherical approximation is made for normal gravity,

$$
\gamma=\left.\frac{\mu}{r^{2}}\right|_{r=R_{\oplus}}=\frac{\mu}{R_{\oplus}^{2}}
$$


The final expression for geoid height becomes,

$$
N=R_{\oplus} \sum_{n=2}^{N_{\max }} \sum_{m=0}^{n} \tilde{P}_{n m}(\sin \phi)\left(\bar{C}_{n m}^{*} \cos m \lambda+\bar{S}_{n m}^{*} \sin m \lambda\right) .
$$

Similarly, changes in the geoid can be expressed,

$$
\Delta N=R_{\oplus} \sum_{n=2}^{N_{\max }} \sum_{m=0}^{n} \tilde{P}_{n m}(\sin \phi)\left(\Delta \bar{C}_{n m} \cos m \lambda+\Delta \bar{S}_{n m} \sin m \lambda\right)
$$

where $\Delta \bar{C}_{n m}$ and $\Delta \bar{S}_{n m}$ are representative of changes in the spherical harmonic coefficients.

It is also common, especially in GRACE applications, to give the disturbing gravitational potential in terms of the water equivalent height. This is done by representing changes to the gravitational potential with a change in surface density. For a full derivation refer to [Wahr et al., 1998]. To summarize, the surface density is calculated with the same set of spherical harmonic coefficients as in Eq. (2.14) with the equation:

$$
\Delta \sigma=\frac{R_{\oplus} \rho_{\oplus}}{3} \sum_{n=2}^{N_{\max }} \sum_{m=0}^{n} \tilde{P}_{n m}(\sin \phi) \frac{2 n+1}{1+k_{n}}\left(\Delta \bar{C}_{n m} \cos m \lambda+\Delta \bar{S}_{n m} \sin m \lambda\right)
$$

where $\rho_{\oplus}$ is the average density of the Earth and $k_{n}$ is the loading Love number for the Earth of degree $n$. The surface mass change in terms of equivalent water height is found by $\Delta \sigma / \rho_{w}$ where $\rho_{w}$ is the density of water. All plots of the gravity field in this report are given in $\mathrm{cm}$ of equivalent water height.

\subsection{Mascons}

Mass concentration blocks (mascons) were first used as a regional gravity estimation technique by Muller and Sjogren [1968]. The technique was developed in an effort to generate a map of the gravity field of the front side of the moon. Since the Deep Space Network can only provide tracking data for lunar orbiters when the spacecraft is 
on the front side of the moon, the mascon technique utilizes only localized tracking data to estimate regional anomalies in the gravity field. Previous to this point, all satellite gravity estimation was done using longer sets of globally distributed data to estimate a set of spherical harmonics.

The same technique can be used for the estimation of local gravity anomalies for any planetary body given a set of sufficient localized satellite tracking data. The GRACE satellite data are especially useful for generating mascon solutions for the Earth. There are several advantages in using a mascon approach over the traditional method of spherical harmonic estimation. One clear advantage of the mascon technique is that global coverage of tracking data is not a requirement as it is with spherical harmonic estimation. Only data over the region of interest is needed. Since only localized data are used to construct the mascon solutions, errors in the force models from a different area of the globe do not affect the solution over the considered area. Another advantage of the mascon approach is that solutions can be constructed with a greater temporal resolution than what is possible with spherical harmonics. This may be especially important as the main thrust of the GRACE and GRACE Follow-On missions is to observe temporal changes in mass distribution in the Earth system. In addition, the mascon approach is significantly less expensive computationally than solving for a full set of spherical harmonics. This is especially important as a GRACE Follow-On mission will likely aim to measure variations in the gravity field at smaller spatial scales than GRACE, for which spherical harmonic solutions would require the use of a supercomputer. Provided that a supercomputer is available, spherical harmonic estimation to a very high degree and order would still require a great deal of processing time.

Recently, a new mascon technique has been developed by Rowlands et al. [2005]. This new approach uses a set of lumped spherical harmonic coefficients to form mascons. 
This method is discussed in Section 2.4.2 while the method of Muller and Sjogren [1968] is explained in Section 2.4.1.

\subsubsection{Point mass and disk mascons}

The work presented in [Muller and Sjogren, 1968] uses a point mass for each mascon. With this approach, point masses are placed at chosen locations on the planetary surface. The acceleration of the spacecraft caused by each point mass is:

$$
\ddot{\mathbf{r}}=-\frac{G m}{r^{3}} \mathbf{r}
$$

where $\mathbf{r}$ is the vector from the point mass to the spacecraft, and $m$ is the mass of the mascon. A least-squares process is then used to find the values of the point masses that best fit the localized spacecraft tracking data. With enough point mass mascons placed on the surface of the planetary body, a regional map of the gravity anomalies can be constructed.

Further investigations into the mascon approach revealed that greater accuracy in the regional gravity anomalies can be achieved by replacing each point mass with a disk [Wong et al., 1971]. The components of acceleration caused by each disk are found by taking the expression for an oblate spheroid, as derived by Moulton [1914], and taking the limit as the thickness approaches zero:

$$
\begin{aligned}
& \ddot{x}=-\frac{3 G m}{2 a^{3}}\left[\frac{-\sqrt{k}}{(1+k)}+\sin ^{-1}\left(\frac{1}{\sqrt{1+k}}\right)\right] x, \\
& \ddot{y}=-\frac{3 G m}{2 a^{3}}\left[\frac{-\sqrt{k}}{(1+k)}+\sin ^{-1}\left(\frac{1}{\sqrt{1+k}}\right)\right] y, \\
& \ddot{z}=-\frac{3 G m}{a^{3}}\left[\frac{1}{\sqrt{k}}-\sin ^{-1}\left(\frac{1}{\sqrt{1+k}}\right)\right] z,
\end{aligned}
$$

where $k$ is the solution of the quadratic:

$$
k^{2} a^{2}+\left[a^{2}-\left(x^{2}+y^{2}+z^{2}\right)\right] k-z^{2}=0,
$$


where $a$ is the radius of the disk, $m$ is the mass of the mascon, and $x, y$, and $z$ are the coordinates of the spacecraft from the center of the disk, with $x$ and $y$ lying in the plane of the disk and $z$ lying along the axis. The design parameters in the setup of mascons includes selecting the locations of the mascons and the disk radius.

As before, a least-squares process is used to find the values of the disk masses that best fit the data. The results can then be used to generate regional maps of gravity anomalies. The mascon solution represents anomalies in the gravity field with respect to some previously defined background gravity field. That background field could be defined as a point mass simplification of the planetary body or a set of background spherical harmonic coefficients.

\subsubsection{Lumped harmonic mascon technique}

The lumped harmonic mascon technique uses a set of differential potential coefficients to represent a small uniform layer of mass over a region at some epoch $t$. The delta coefficients are computed by Chao et al. [1987]:

$$
\Delta \bar{A}_{n m}=\frac{\left(1+k_{n}^{\prime}\right) R_{\oplus}^{2} \sigma(t)}{(2 n+1) M} \int Y_{n m}(\Omega) d \Omega
$$

where $n$ and $m$ are the spherical degree and order, respectively, $k_{n}^{\prime}$ is the load Love number of degree $n, R_{\oplus}$ is the Earth radius, $\Omega$ is a representation of surface area, $Y_{n m}$ is the spherical harmonic of degree $n$ and order $m$, and $\sigma(t)$ is the mass of the layer over a unit of surface area at epoch $t$.

Once the size and shape of the mascons are selected, Eq. (2.18) is used to generate the set of delta coefficients that corresponds to $1 \mathrm{~cm}$ of water over each mascon. The mascon estimate is then a scale factor of that set of delta coefficients, and is representative of how much the gravitational signal varies with respect to the background 
field over that mascon in units of $\mathrm{cm}$ of water.

A least-squares estimation procedure is used to find the estimates of the mascon parameters. The partial derivatives of the tracking observations with respect to the mascon parameters are equal to a linear combination of the partial derivatives of the tracking observations with respect to the spherical harmonic coefficients, multiplied by the delta coefficients for that mascon [Luthcke et al., 2008]:

$$
\frac{\partial O_{i}}{\partial P_{t}^{j}}=\sum_{n=2}^{N_{\max }} \sum_{m=0}^{n}\left(\frac{\partial O_{i}}{\partial \bar{C}_{n m}} \Delta \bar{C}_{n m}^{j}+\frac{\partial O_{i}}{\partial \bar{S}_{n m}} \Delta \bar{S}_{n m}^{j}\right),
$$

where $\partial O_{i} / \partial P_{t}^{j}$ is the partial derivative of the range-rate observation $i$ with respect to the mascon $P^{j}$ at time $t, \partial O_{i} / \partial \bar{C}_{n m}$ and $\partial O_{i} / \partial \bar{S}_{n m}$ are the partial derivatives of the range-rate observation with respect to the spherical harmonic coefficients, and $\Delta \bar{C}_{n m}$ and $\Delta \bar{S}_{n m}$ are found using Eq. (2.18).

It is often useful to apply constraints to the mascon estimation procedure. Both spatial and temporal constraints can be placed on the solution by introducing a constraint matrix into the system of equations. The constraint matrix constrains each distinct pair of mascons to each other, resulting in a symmetric $p \times p$ matrix, where $p$ is the number of estimated mascons. The components of the symmetric matrix are given by:

$$
C_{i j}=\exp \left[2-\frac{d_{i j}}{D}-\frac{\left|t_{i j}\right|}{T}\right],
$$

where $D$ and $T$ are the correlation distance and correlation time respectively, $d_{i j}$ is the distance between blocks $i$ and $j$, and $t_{i j}$ is the difference in time tags for block $i$ and $j$ (note that $i$ and $j$ in the above equation are not the same as in Eq. (2.19)). The correlation distance and time are design parameters of the solution and are selected based on the strength of the data and the size of the mascon blocks.

Improved mascon solutions are achieved by forming separate constraint matrices 
for locations which have unrelated geophysical signals. For example, if land and ocean mascons are constrained separately, the result is a land constraint matrix with dimensions $q \times q$ and an ocean constraint matrix with dimensions $r \times r$, where $q$ and $r$ are the number of land and ocean mascons respectively, and $r+q=p$. The $C$ matrix is thus block diagonal and still is of dimension $p \times p$.

It is important to note that since only localized tracking data are used to generate mascon solutions, the longer wavelength gravity field signals may not be well recovered. To mitigate this issue, a surface area larger than the actual area of interest is selected and the solution at the edges is discarded.

This mascon technique has proven successful in estimating variations in ice mass over Greenland and in the Gulf of Alaska with a relatively high spatial and temporal resolution [Luthcke et al., 2006, 2008]. The lumped harmonic mascon technique is used throughout this work.

\subsection{Averaging Kernels}

Regional estimates of mass content can be extracted from spherical harmonic solutions with averaging kernels. Given a time series of spherical harmonic gravity estimates, an averaging kernel can be used to form a time series of mass variation for some selected region or basin. This data set is often then used to calculate the trend and annual signal for the region. This approach only applies to determining mass variations for sets of spherical harmonics since the mascon technique of Section 2.4.2 allows for the mass changes to be directly summed. The method summarized in this section is fully developed in [Swenson and Wahr, 2002].

An exact averaging kernel, $\vartheta(\phi, \lambda)$, describes the shape of the basin to be inves- 
tigated, and is defined by:

$$
\vartheta(\phi, \lambda)=\left\{\begin{array}{l}
0 \text { outside the basin } \\
1 \text { inside the basin }
\end{array}\right.
$$

The mean of the vertically integrated mass change for a given region is:

$$
\overline{\Delta \sigma}_{\text {region }}=\frac{1}{\Omega_{\text {region }}} \int \Delta \sigma(\phi, \lambda) \vartheta(\phi, \lambda) d \Omega
$$

where $d \Omega$ is an element of solid angle and $\Omega_{\text {region }}$ is calculated by integrating $\vartheta(\phi, \lambda)$ over the sphere. Using Eq. (2.15), Eq. (2.22) can be expressed as a sum of spherical harmonic coefficients:

$$
\overline{\Delta \sigma}_{\text {region }}=\frac{R_{\oplus} \rho_{\oplus}}{3 \Omega_{\text {region }}} \sum_{n=0}^{\infty} \sum_{m=0}^{n} \frac{2 n+1}{1+k_{n}}\left(\vartheta_{n m}^{C} \Delta \bar{C}_{n m}+\vartheta_{n m}^{S} \Delta \bar{S}_{n m}\right)
$$

where $\vartheta_{n m}^{C}$ and $\vartheta_{n m}^{S}$ are the spherical harmonic coefficients which describe $\vartheta(\phi, \lambda)$ :

$$
\begin{gathered}
\vartheta(\phi, \lambda)=\frac{1}{4 \pi} \sum_{n=0}^{\infty} \sum_{m=0}^{n} \tilde{P}_{n m}(\sin \phi)\left(\vartheta_{n m}^{C} \cos m \lambda+\vartheta_{n m}^{S} \sin m \lambda\right) \\
\left\{\begin{array}{c}
\vartheta_{n m}^{C} \\
\vartheta_{n m}^{S}
\end{array}\right\}=\int \vartheta(\phi, \lambda) \tilde{P}_{n m}(\sin \phi)\left\{\begin{array}{c}
\cos m \lambda \\
\sin m \lambda
\end{array}\right\} d \Omega .
\end{gathered}
$$

The calculated mass within a given basin will differ from the truth due to satellite measurement error and truncation error. Satellite measurement error is the result of the sum of error sources which affect any gravity recovery mission as described in detail in Chapter 4. Truncation error exists because the spherical harmonic coefficients which describe the basin are not expanded to infinity and are thus not a perfect representation of the shape of the basin. The maximum degree to which the basin harmonics are formed is limited by the size of the spherical harmonic expansion which describes the estimated gravity field as provided by GRACE or a GFO. The estimated average mass within the region as it relates to these error sources is defined by:

$$
\overline{\Delta \sigma}_{\text {region }}^{\text {GRACE }}=\overline{\Delta \sigma}_{\text {region }}^{\text {truth }}+\overline{\Delta \sigma}_{\text {region }}^{\text {satellite error }}+\overline{\Delta \sigma}_{\text {region }}^{\text {truncation error }}
$$


The magnitude of the satellite error increases with a higher truncation degree while the truncation error increases with a smaller truncation degree.

The exact averaging kernel, $\vartheta(\phi, \lambda)$, contains short-wavelength coefficients due to the fact that it changes discontinuously from a value of 1 to 0 at the border of the selected basin. Since the satellite errors are largest for high degree $n$, it is advantageous to use a smoothed averaging kernel, $\bar{W}(\phi, \lambda)$, in place of the exact averaging kernel, $\vartheta(\phi, \lambda)$, to obtain a more accurate estimate of the average mass within the basin. Substituting $\bar{W}(\phi, \lambda)$ for $\vartheta(\phi, \lambda)$ in Eq. (2.22), the approximate basin average is given by:

$$
\widetilde{\Delta \sigma_{\text {region }}}=\frac{1}{\Omega_{\text {region }}} \int \Delta \sigma(\phi, \lambda) \bar{W}(\phi, \lambda) d \Omega
$$

As before, Eq. (2.27) can be expressed as a sum of spherical harmonic coefficients:

$$
\widetilde{\Delta \sigma}_{\text {region }}=\frac{R_{\oplus} \rho_{\oplus}}{3 \Omega_{\text {region }}} \sum_{n=0}^{\infty} \sum_{m=0}^{n} \frac{2 n+1}{1+k_{n}}\left(W_{n m}^{C} \Delta \bar{C}_{n m}+W_{n m}^{S} \Delta \bar{S}_{n m}\right),
$$

where $W_{n m}^{C}$ and $W_{n m}^{S}$ describe $\bar{W}(\phi, \lambda)$ :

$$
\bar{W}(\phi, \lambda)=\frac{1}{4 \pi} \sum_{n=0}^{n_{t r n c}} \sum_{m=0}^{n} \tilde{P}_{n m}(\sin \phi)\left(W_{n m}^{C} \cos m \lambda+W_{n m}^{S} \sin m \lambda\right) .
$$

In this study, a Gaussian smoothing function was used to construct the averaging kernel. For a Gaussian smoothed averaging kernel the weighting coefficients in Eq. (2.28) are defined by:

$$
\left\{\begin{array}{c}
W_{n m}^{C} \\
W_{n m}^{S}
\end{array}\right\}=2 \pi W_{n}\left\{\begin{array}{c}
\vartheta_{n m}^{C} \\
\vartheta_{n m}^{S}
\end{array}\right\}
$$

where

$$
W_{n}=\frac{1}{\sqrt{2 n+1}} \int_{0}^{\pi} W(\gamma) \tilde{P}_{n 0}(\cos \gamma) \sin \gamma d \gamma
$$

and

$$
\begin{aligned}
W(\gamma) & =\frac{b}{2 \pi} \frac{\exp [-b(1-\cos \gamma)]}{1-e^{-2 b}}, \\
b & =\frac{\ln (2)}{\left(1-\cos \left(r_{1 / 2} / R_{\oplus}\right)\right)}
\end{aligned}
$$


and $r_{1 / 2}$ is the approximate distance at which the averaging kernel changes from a value of 1 inside the basin to 0 outside the basin.

Since the value of a smoothed averaging kernel changes gradually from a value of 1 inside the basin to 0 outside the basin, some undesired signal that is outside of the basin will affect the estimate of the basin mass average. This problem is referred to as leakage. Another effect of the smoothed averaging kernel is that the magnitude of the estimated mass signal is somewhat dampened by the smoothing. In order to account for this effect, a scale factor must be applied to the solution. This scale factor can be determined by generating a set of spherical harmonics, which represent a uniform mass over the selected basin, and then recovering that simulated signal with the smoothed averaging kernel. The scale factor is selected such that the recovered mass exactly matches the total mass present for the uniform mass representation of the basin.

\subsection{Methods for Analyzing the Accuracy of Gravity Estimates}

This section summarizes the methods used in this study to determine the relative accuracies of the gravity estimates and compare the various simulated mission configurations.

The root-mean-square (RMS) is a statistical tool that determines the magnitude of variations of some quantity $\epsilon$, and is defined by:

$$
\operatorname{RMS}=\sqrt{\frac{1}{n} \sum_{i=0}^{n} \epsilon_{i}^{2}} .
$$

In this study the RMS is used to quantify the errors of the simulated gravity estimates in two different ways. First, the $1^{\circ} \times 1^{\circ}$ local truth and estimated spatial maps are differenced to form a local spatial map that defines the error. The values of the error

grid define $\epsilon$ and the RMS of the spatial error map is calculated. Second, a time series 
of water mass changes for given basins are calculated with a series of gravity estimates. The truth mass changes are differenced with the estimated mass changes to form a time series of errors in the mass change estimates. This time series defines $\epsilon$ and determines the RMS of the mass change estimates.

It is desirable to have some method for presenting the errors as a function of spatial frequency. For full spherical harmonic expansions describing the global gravity field, the geoid degree variance is often calculated:

$$
\operatorname{RMS}\left(N_{n}\right)=R_{\oplus} \sqrt{\frac{\sum_{m=0}^{n}\left(C_{n m}^{2}+S_{n m}^{2}\right)}{2 n+1}},
$$

where $R_{\oplus}$ is the radius of the Earth, and $C_{n m}$ and $S_{n m}$ are the harmonic coefficients. The harmonic coefficients here could describe either the estimated coefficients to show the power of the estimated signal at each degree, or the error coefficients to observe the spatial frequency characteristics of the errors. Since most of the gravity estimates in this study are formed with the mascon regional gravity estimation method this method of comparison will not be of use.

A method used by Han et al. [2005] determines the frequency characteristics of a local spatial map. The procedure is summarized by two steps: 1.) calculate the two dimensional spectral density of a localized spatial map, and 2.) sample the two dimensional spectral density in azimuth to obtain the power at each sampled frequency. The first step assumes that the map is planar and the second step assumes an isotropic spectral density. Both assumptions are simplifications of the problem, but the results do provide good spectral information of the original spatial plot. The first step of the procedure is implemented by calculating the two dimensional discrete Fourier transform given by Eq. (B.10) and then determining the two dimensional spectral density with 
Eq. (C.3).

Figure 2.6 provides a few examples of spatial maps and their corresponding two dimensional spectral densities to illustrate the concept of the described technique. The example spatial maps contain signals at frequencies which are perfectly sampled by the Fourier transform. The white dots on the spectral maps are high values and show the frequencies which are present in the spatial map. Frequencies in spatial maps that are not sampled by the Fourier transform introduce an error called spectral leakage, which affects the spectral density at the sample frequencies.

As previously mentioned, the gravity estimates are used to generate time series of mass changes for given basin locations. With the lengthy time series generated in this report (25 months) it is possible to estimate some key variables of the mass change, specifically trend, amplitude of the annual signal, and phase. This is done by performing a least squares fit to the data by estimating a constant, trend, amplitude of annual signal, and a phase for both the truth signal and the estimated signal. The estimates for the various mission configurations can then be compared to the truth to determine their ability to measure these values. 

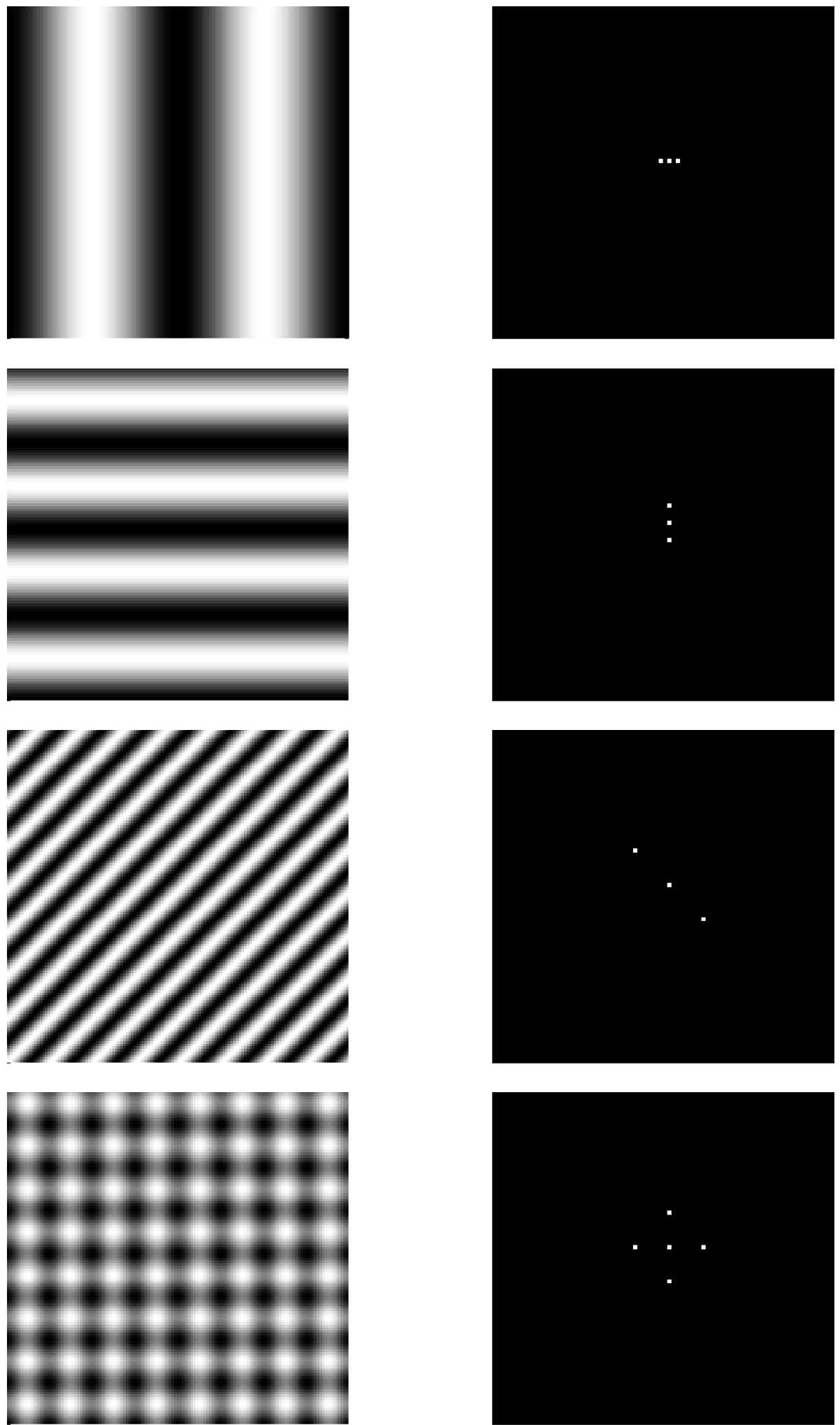

Figure 2.3: Examples of two dimensional spectral densities. Left column: spatial maps with different two dimensional cosine functions, Right column: corresponding two dimensional spectral density. 


\section{Chapter 3}

\section{Simulation Models and Procedure}

\subsection{Introduction}

This chapter describes the models and procedure used for the time-variable gravity recovery numerical simulations. The simulation procedure is summarized by three steps 1.) generate simulated satellite-to-satellite range-rate data and satellite position

data, 2.) estimate satellite state initial conditions and satellite accelerations, and 3.) estimate the gravity field and adjust the satellite state. All numerical simulations are performed using the GEODYN orbit determination software and SOLVE large linear systems solver, which have been made available by NASA Goddard Space Flight Center.

Contained in this chapter is a description of the force models, the linear estimation theory implemented by the simulation software, the gravity recovery simulation procedure, and the mission orbital parameters, which are used for GRACE and the GFO missions. The simulation procedure describes the inclusion of all major error sources for time-variable gravity estimation which are fully explained in Chapter 4. 


\section{$3.2 \quad$ Force Models}

The motion of an Earth orbiting satellite is described by the differential equation:

$$
\ddot{\mathbf{r}}=-\frac{\mu}{r^{3}} \mathbf{r}+\mathbf{f}
$$

where $\mathbf{r}$ is the geocentric position vector of the spacecraft, and $\mathbf{f}$ is the sum of the perturbing forces as given by:

$$
\mathbf{f}=\mathbf{f}_{N S}+\mathbf{f}_{N B}+\mathbf{f}_{g}+\mathbf{f}_{D}+\mathbf{f}_{S R P}+\mathbf{f}_{E R P}+\mathbf{f}_{O t h e r},
$$

where

$\mathbf{f}_{N S}$ is caused by the non-spherical gravity field,

$\mathbf{f}_{N B}$ is the result of N-body perturbations, primarily from the Sun and Moon,

$\mathbf{f}_{g}$ is the contribution from general relativity,

$\mathbf{f}_{D}$ is the forcing from atmospheric drag,

$\mathbf{f}_{S R P}$ is forcing from solar radiation pressure,

$\mathbf{f}_{E R P}$ is forcing from Earth radiation pressure,

$\mathbf{f}_{\text {Other }}$ is the total contribution of all other forces.

The term $\mathbf{f}_{N S}$ is composed of the static gravity field, the Earth and ocean tides, the atmospheric and hydrological signals, and the response of the solid Earth to these time-variable loadings. This force can be calculated as the gradient of the potential $U$ :

$$
\mathbf{f}_{N S}=\nabla U
$$

where

$$
U=\frac{\mu}{r} \sum_{n=2}^{\infty} \sum_{m=0}^{n}\left(\frac{R_{\oplus}}{r}\right)^{n} \tilde{P}_{n m}(\sin \phi)\left(\bar{C}_{n m}(t) \cos m \lambda+\bar{S}_{n m}(t) \sin m \lambda\right) .
$$

Here, $\bar{C}_{n m}(t)$ and $\bar{S}_{n m}(t)$ are time-dependent normalized geopotential coefficients which are used to describe all variations in the gravity field. The accurate determination of 
the time-varying component of the gravitational signal is the primary goal of GRACE and a GFO mission.

The $\mathbf{f}_{N B}$ and $\mathbf{f}_{g}$ terms are well understood and their affects on satellite motion are modeled and removed prior to gravity estimation. The terms $\mathbf{f}_{D}, \mathbf{f}_{S R P}$, and $\mathbf{f}_{E R P}$ are non-conservative forces. The accelerations on the satellite caused by these components are measured by GRACE with the on-board accelerometer. The accelerometer data are then used to separate these forces from the conservative forces caused by variations in the gravity field. A GFO mission may be equipped with a drag-free system, for which thrusting is used to counteract the non-conservative forces. Appropriate errors for the on-board accelerometer and the drag-free system are included in the numerical simulations and are described in detail in Chapter 4. The drag-free system is also referred to as the gravitational reference sensor (GRS).

As explained in Section 1.2, several of the geophysical processes, which make up the $\mathbf{f}_{N S}$ term, are modeled and removed prior to the estimation of the gravity field. Errors in the models used to remove these signals can cause significant errors in the gravity estimates for GRACE. The inclusion of force model errors in this simulation study is required for a realistic assessment of GRACE and GFO mission performance. To simulate these errors, Truth force models are used to simulate truth data and Nominal force models are used in the gravity estimation. The difference between the models is then considered the level of uncertainty for that particular geophysical process. This procedure, which assumes that the differences between two different geophysical models represents the error of that model, has been a common practice for GRACE simulation studies [Han et al., 2004; Seo et al., 2008a; Seo and Wilson, 2005; Thompson et al., 2004]. These model errors introduce a significant error source into the gravity solutions known as temporal aliasing which is discussed in Section 4.5. A table summarizing the 
force models used in the simulations is given in Table 3.1 .

\begin{tabular}{|l|l|l|}
\hline & Truth & Nominal \\
\hline Background Field & EIGEN-GL04C & EIGEN-GL04C \\
\hline Tidal & FES2004 & GOT00 \\
\hline Atmospheric & ECMWF & NCEP \\
\hline Oceanographic & OMCT & MOG2D \\
\hline
\end{tabular}

Table 3.1: Force models used for numerical simulations.

The background gravity field, EIGEN-GLO4C is an estimate of the static gravity field found using GRACE and LAGEOS mission data, altimeter data, and gravimetry measurements. For the simulations in this work, the same background field is used for the Truth and Nominal models, meaning that the errors in the static gravity field are not included. The static field errors are excluded for two reasons: 1.) The focus of this work is the recovery of time-variable gravity, which will be largely unaffected by errors in the static field as those errors will be identical from one time step to the next, and 2.) The errors in the static field are quite large at a high spatial resolution and would be the dominant feature in all of the estimated time-variable gravity maps presented in this work. Additionally, it is also a possibility that the GOCE satellite mission will provide the static portion of the gravity field with unprecedented accuracy prior to the launch of a GFO, thus minimizing this source of error.

The finite element solution (FES) tidal models are computed using the tidal hydrodynamic equations assimilated with tide gauge and TOPEX/Poseidon data. The process is explained by Lefèvre [2002]. The Goddard Ocean Tide (GOT) model is an assimilated model computed from years of the TOPEX/Poseidon data and is developed by Ray [1999]. 
The atmospheric estimates are provided by the European Centre for MediumRange Weather Forecasts (ECMWF) and the National Centers for Environmental Prediction (NCEP) and are formed using a global network of meteorological stations. The oceanographic component is due to meteorological forcing which causes changes in ocean heights that are not part of the tidal signal. The most simplistic representation of this signal is the inverted barometer (IB) affect. The IB assumption states that a change in atmospheric pressure is balanced by a corresponding change in ocean height and does not take into account wind effects [Wunsch and Stammer, 1997]. For the IB assumption a 1 mbar increase in atmospheric pressure results in a $\sim 1 \mathrm{~cm}$ decrease in ocean height. The OMCT (Ocean Model for Circulation and Tides) and MOG2D-G (2D Gravity Waves model) oceanographic models more accurately model the high-frequency oceanic variations caused by meteorological forcing with the use of hydrodynamic equations. Refer to [Flechtner, 2007] and [Carrère and Lyard, 2003] respectively for detailed descriptions of the OMCT and MOG2D-G oceanic models. The atmospheric and oceanographic models are summed and input into GEODYN as 3-hourly sets of spherical harmonics. The Truth model is the sum of ECMWF and OMCT which is directly available as the GRACE AOD1B (atmosphere and ocean de-aliasing level-1b) product from <ftp://podaac.jpl.nasa.gov/grace/>. The sum of NCEP and MOG2D is used as the Nominal set of models. It should be noted that the atmospheric tides are present in the ECMWF/OMCT model but are not present in the NCEP/MOG2D model. For this reason the atmospheric tides are removed from the FES2004 tidal model but not from the GOT00 model.

The sum of the atmospheric and oceanographic components are usually referred to as AOD (Atmosphere and Ocean De-aliasing). The AOD Truth and Nominal mass variation signals for the Amazon basin and Greenland for 2003 are shown in Figure 3.1(a) 
and Figure 3.1(b) respectively. The AOD error for the two locations (the difference between the Truth and Nominal models) is plotted in Figure 3.2. Maps of the mean AOD monthly errors for South America and Greenland are plotted in Figure 3.3.

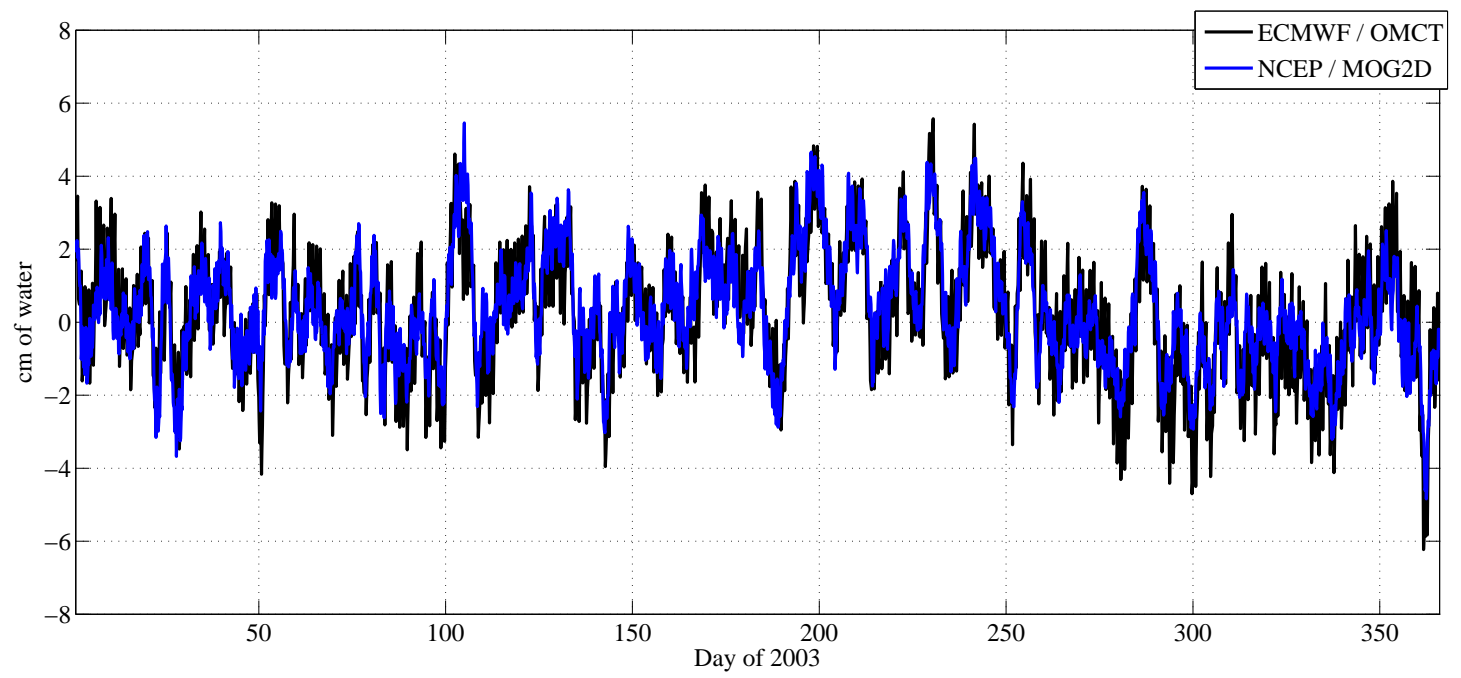

(a) Amazon

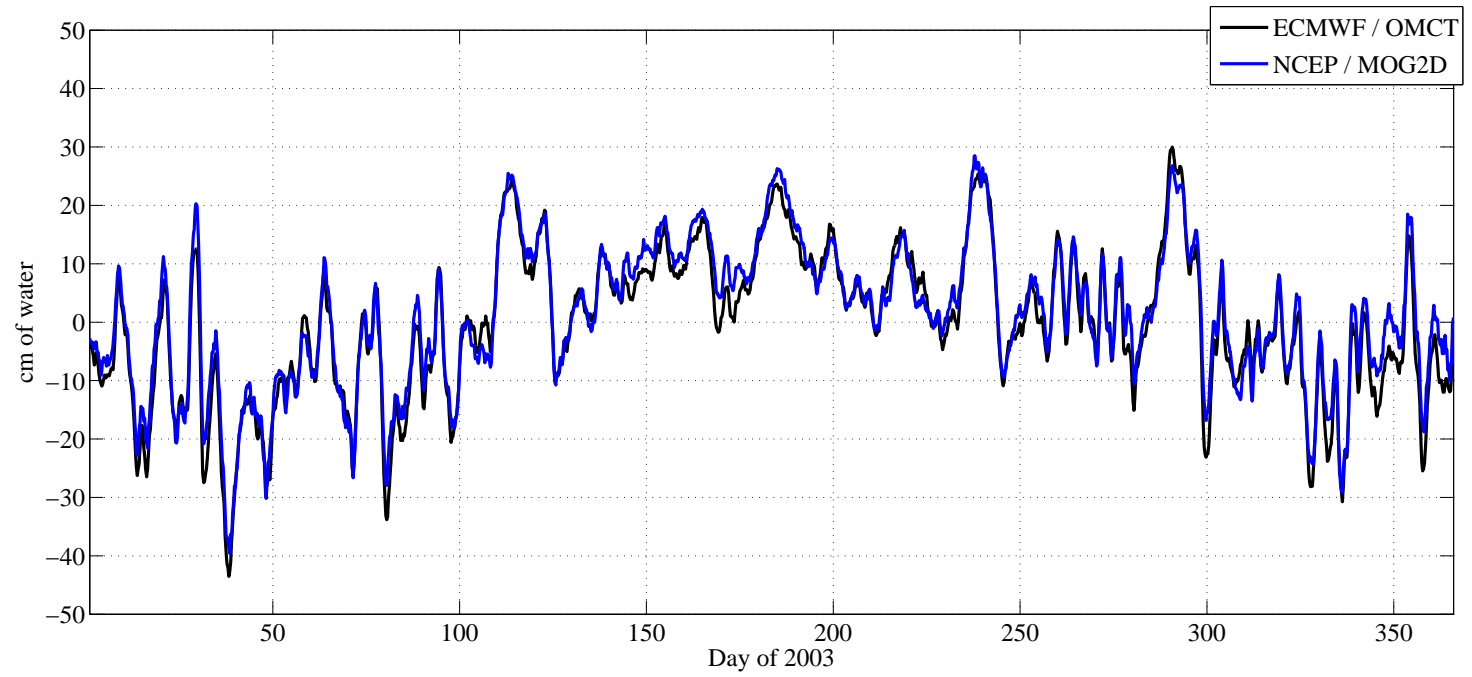

(b) Greenland

Figure 3.1: AOD signal mass variations for 2003. 


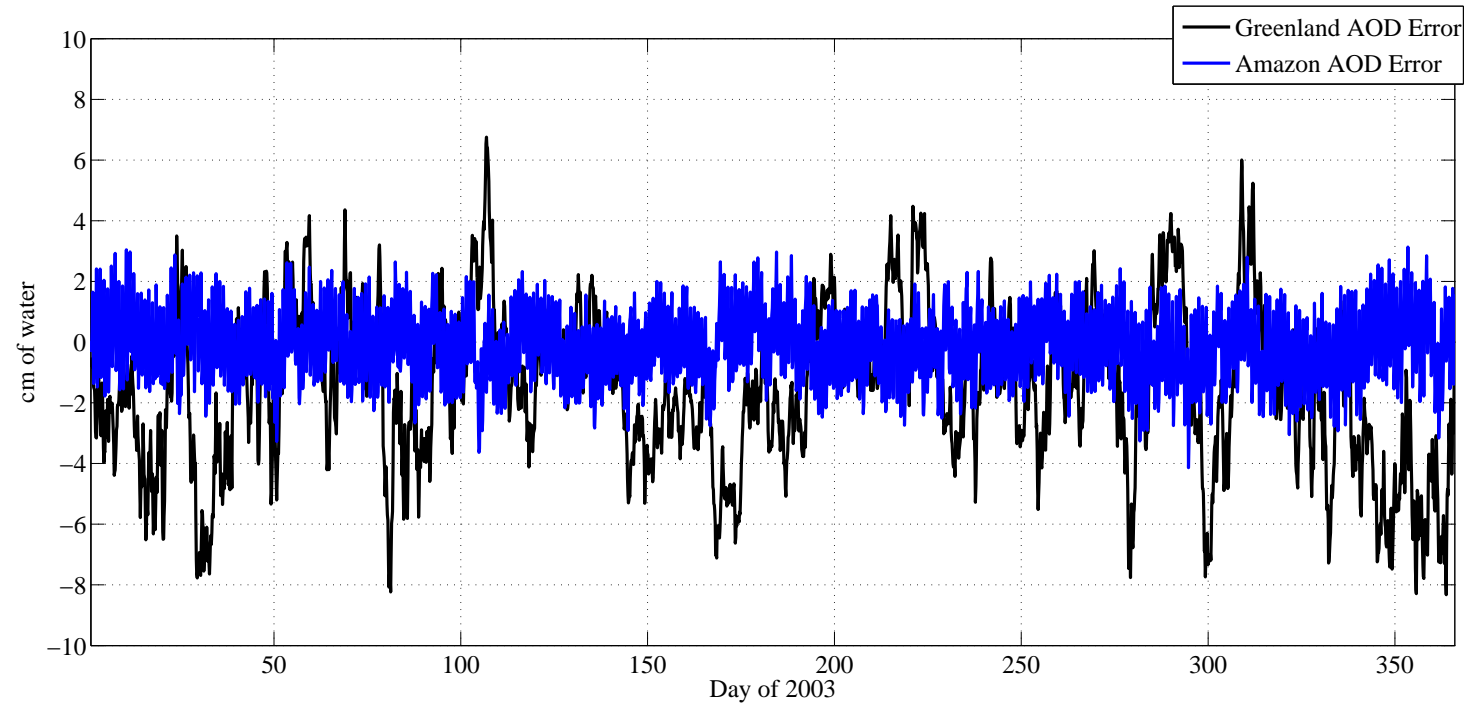

Figure 3.2: AOD errors for Amazon basin and Greenland mass variations for 2003. 

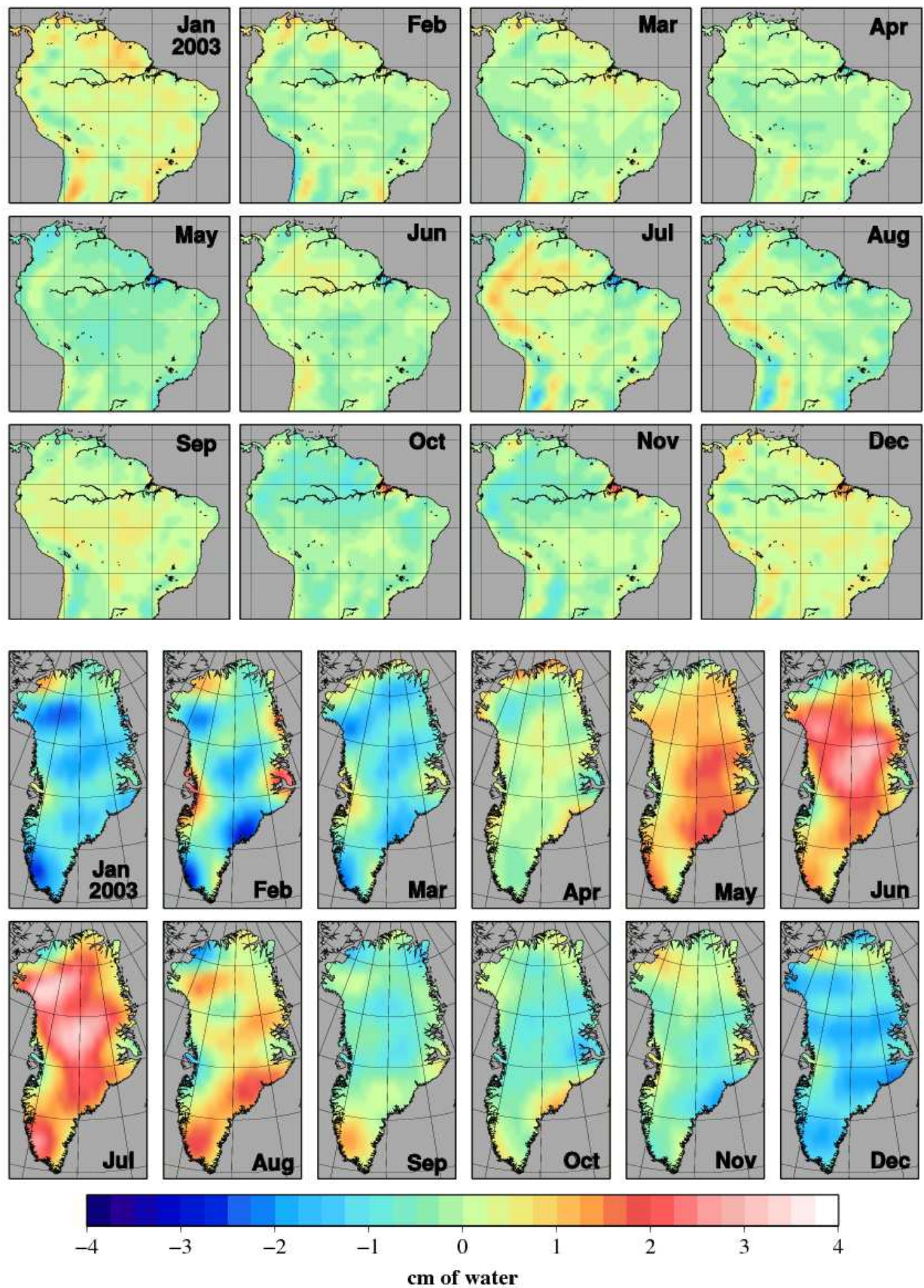

Figure 3.3: Monthly means of South America and Greenland AOD model error (ECMWF/OMCT minus NCEP/MOG2D). The twelve month mean has been removed. 
In order to effectively determine the ability of a GRACE Follow-On mission to recover the gravity field, it is necessary to estimate the time-variable gravity field at multiple locations on the Earth's surface. In this study, gravity estimates are simulated for Greenland and the Amazon and surrounding basins of South America. These locations were chosen because of their interest to the scientific community and because they are located at different latitudinal bands, resulting in different groundtrack coverage for the two locations for both GRACE and GFO.

The Truth signal, which is estimated by the numerical simulations over South America is the Global Land Data Assimilation System (GLDAS) hydrology model developed by Rodell [2004b]. As with the time-varying atmospheric and oceanic signal, the GLDAS Truth signal is input into GEODYN as 3-hourly sets of spherical harmonics. The monthly means of twenty four months of GLDAS data are shown in Figure 3.4. These spatial plots represent the Truth signal for South America and are used to determine the level of error for the estimates obtained for the GRACE and GFO simulations. The Truth GLDAS signal is included up to spherical harmonic degree and order 72 .

A model for mass change over Greenland is not readily available, so a Truth model is constructed. The Truth model for Greenland is a $1^{\circ} \times 1^{\circ}$ grid for which each block is assigned a mass trend value and an amplitude of the annual signal. The amplitude of the annual signal is dependent on the elevation of the block. These monthly Truth grids are converted into spherical harmonics prior to being used as input for GEODYN with an expansion to degree and order 180 . The mass trend values for the $1^{\circ} \times 1^{\circ}$ blocks range from an increase of $\sim 0.2 \mathrm{GT} /$ year to a mass loss of of $\sim 4.7 \mathrm{GT} /$ year. The amplitude of the annual signal for the region below $2000 \mathrm{~m}$ elevation is 300 GT while the region above $2000 \mathrm{~m}$ has an amplitude of $50 \mathrm{GT}$. 

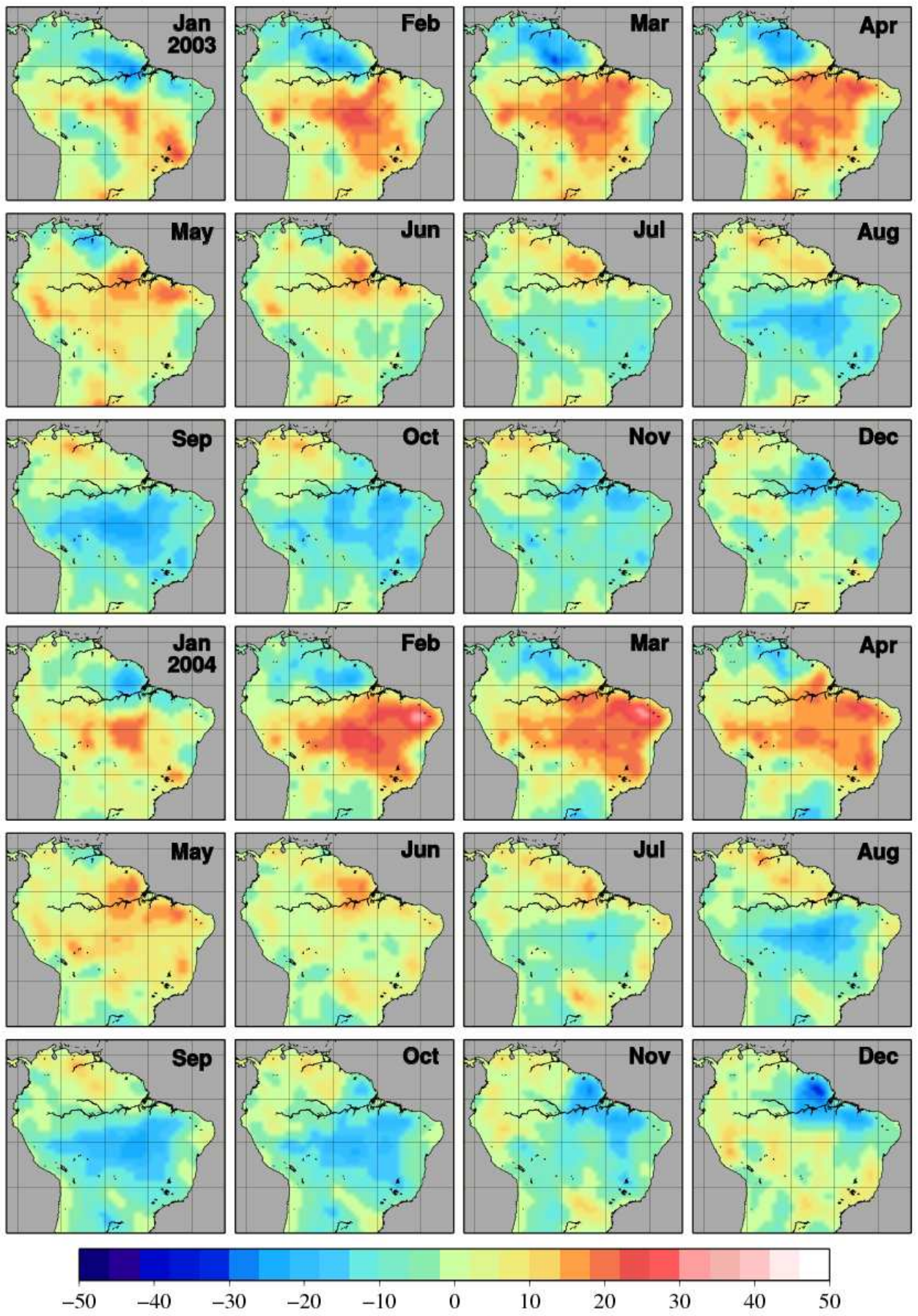

cm of water

Figure 3.4: Truth signal for South America over 24-month simulation (Jan, 2003 to Dec, 2004). 
Figure 3.5 is the spherical harmonic map of mass trend and Figure 3.6 shows the Greenland locations which are above and below $2000 \mathrm{~m}$. The resulting Greenland Truth signal is the sum of the mass trend and the annual signal and is shown in Figure 3.7.

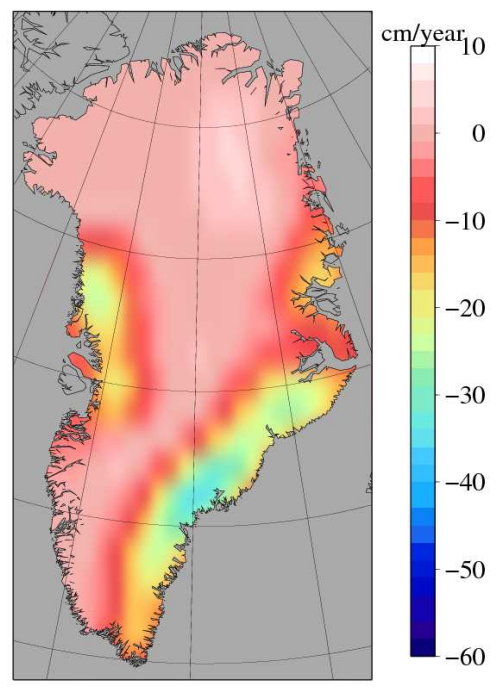

Figure 3.5: Map of truth mass trends for constructed Greenland model.

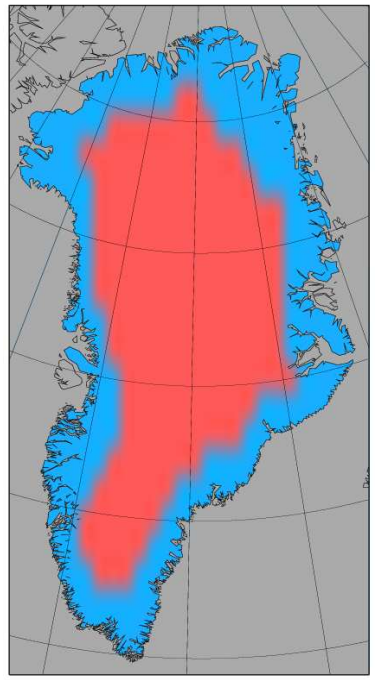

Figure 3.6: Blue section: elevations below $2000 \mathrm{~m}$ are assigned an annual amplitude of 300 GT. Red section: elevations greater than $2000 \mathrm{~m}$ are assigned and annual amplitude of $50 \mathrm{GT}$. 

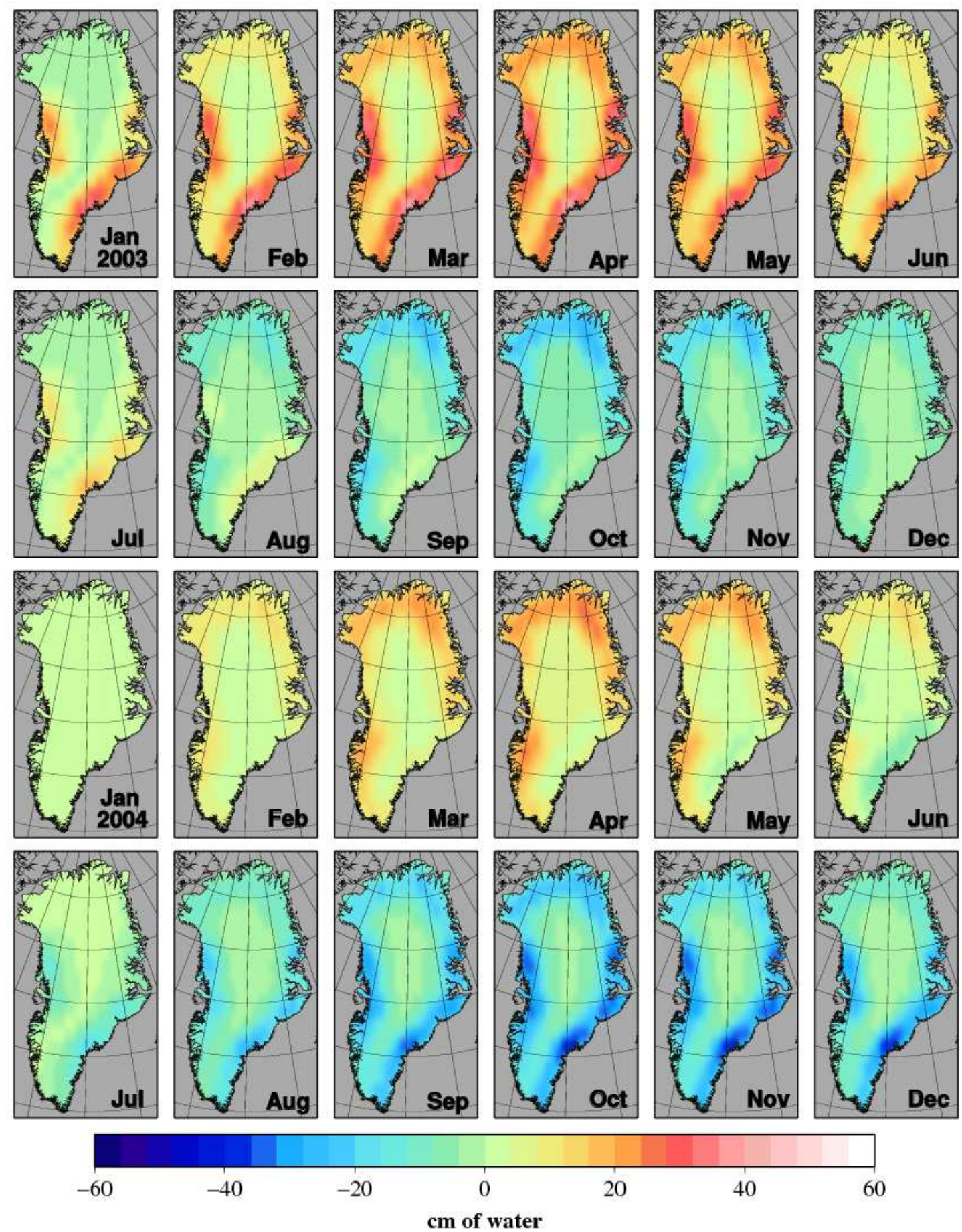

Figure 3.7: Truth signal for Greenland over 24-month simulation (Jan, 2003 to Dec, 2004). 


\subsection{Linear Estimation Theory}

A weighted least squares estimation procedure is implemented in the GEODYN and SOLVE software packages used for the numerical simulations. This section summarizes the linear estimation theory. For a more detailed derivation of the equations presented here, refer to [Tapley et al., 2004c].

The state vector is composed of all variables to be estimated, and can be defined as,

$$
\mathbf{X}=\left[\begin{array}{lll}
\mathbf{r} & \mathbf{v} & \boldsymbol{\alpha}
\end{array}\right]^{T},
$$

where $\mathbf{r}$ and $\mathbf{v}$ are the position and velocity vectors respectively, and $\boldsymbol{\alpha}$ consists of the constant model parameters. For the simulations performed in this study $\boldsymbol{\alpha}$ is made up of either the satellite general accelerations or the gravity parameters (i.e. spherical harmonic coefficients or mascons).

The nonlinear equations describing the dynamics of the state are given as

$$
\dot{\mathbf{X}}=F(\mathbf{X}, t) \quad \mathbf{X}\left(t_{k}\right) \equiv \mathbf{X}_{k}
$$

where $\mathbf{X}_{k}$ is the $n$-dimensional state vector at time $t_{k}$. The observation equation is represented as

$$
\mathbf{Y}_{i}=G\left(\mathbf{X}_{i}, t_{i}\right)+\boldsymbol{\epsilon}_{i} \quad i=1, \ldots, l
$$

where each $\mathbf{Y}_{i}$ is a set of $p$ observations at time $t_{i}, G\left(\mathbf{X}_{i}, t_{i}\right)$ is a nonlinear relationship between the state vector and the observation, and $\boldsymbol{\epsilon}_{i}$ is representative of the measurement noise.

The nonlinear state and observation equations are then linearized in the following way. First, the $n \times 1$ state deviation vector $\mathrm{x}$ and the $p \times 1$ observation deviation vector 
$\mathbf{y}$ are defined:

$$
\mathbf{x}(t)=\mathbf{X}(t)-\mathbf{X}^{*}(t) \quad \mathbf{y}(t)=\mathbf{Y}(t)-\mathbf{Y}^{*}(t),
$$

where $\mathbf{X}$ is the true state and $\mathbf{X}^{*}$ is the reference state. A first-order Taylor series expansion can then be performed on Eq. (3.6) and Eq. (3.7) to arrive at:

$$
\begin{aligned}
\dot{\mathbf{x}}(t) & =A(t) \mathbf{x}(t) \\
\mathbf{y}_{i} & =\tilde{H}_{i} \mathbf{x}_{i}+\boldsymbol{\epsilon}_{i} \quad(i=1, \ldots, l)
\end{aligned}
$$

where

$$
A(t)=\frac{\partial F}{\partial \mathbf{X}}\left(\mathbf{X}^{*}, t\right) \quad \tilde{H}_{i}=\frac{\partial G}{\partial \mathbf{X}}\left(\mathbf{X}^{*}, t\right)
$$

The general solution for Eq. (3.9) can be expressed as

$$
\mathbf{x}(t)=\Phi\left(t, t_{k}\right) \mathbf{x}_{0}
$$

where $\Phi\left(t, t_{k}\right)$ is the state transition matrix and $\mathbf{x}_{k}$ is the state deviation vector at time $t_{k}$. The state transition matrix satisfies the following differential equation:

$$
\Phi\left(t, t_{k}\right)=A(t) \dot{\Phi}\left(t, t_{0}\right) \quad \Phi\left(t_{k}, t_{k}\right)=I
$$

and can be solved for at any time, $t$, with numerical integration.

Combining Eq. (3.12) and Eq. (3.10), the observations can be related to a single epoch of the state:

$$
\mathbf{y}=H \mathbf{x}_{k}+\boldsymbol{\epsilon}
$$

where

$$
\mathbf{y}=\left[\begin{array}{c}
\mathbf{y}_{1} \\
\mathbf{y}_{2} \\
\vdots \\
\mathbf{y}_{m}
\end{array}\right] \quad H=\left[\begin{array}{c}
\tilde{H}_{1} \Phi\left(t_{1}, t_{k}\right) \\
\tilde{H}_{2} \Phi\left(t_{2}, t_{k}\right) \\
\vdots \\
\tilde{H}_{m} \Phi\left(t_{m}, t_{k}\right)
\end{array}\right] \quad \boldsymbol{\epsilon}=\left[\begin{array}{c}
\boldsymbol{\epsilon}_{1} \\
\boldsymbol{\epsilon}_{2} \\
\vdots \\
\boldsymbol{\epsilon}_{m}
\end{array}\right]
$$


where, $\mathbf{y}$ is an $m \times 1$ vector, $\mathbf{x}_{k}$ is an $n \times 1$ vector, $\boldsymbol{\epsilon}$ is an $n \times 1$ vector, $H$ is an $m \times n$ mapping matrix, and $m=p \times l$ and is the total number of observations where $p$ is the number of observations per epoch and $l$ is the number of epochs.

The weighted least squares solution uses a weighting matrix $W$ which describes the statistical properties of the observations. If an a priori state deviation vector $\overline{\mathbf{x}}_{k}$ is available with weighting matrix $\bar{W}$, then the weighted least squares solution is found by minimizing the performance index:

$$
J\left(\mathbf{x}_{k}\right)=1 / 2\left(\mathbf{y}-H \mathbf{x}_{k}\right)^{T} W\left(\mathbf{y}-H \mathbf{x}_{k}\right)+1 / 2\left(\overline{\mathbf{x}}_{k}-\mathbf{x}_{k}\right)^{T} \bar{W}_{k}\left(\overline{\mathbf{x}}_{k}-\mathbf{x}_{k}\right)
$$

which results in:

$$
\hat{\mathbf{x}}_{k}=\left(H^{T} W H+\bar{W}_{k}\right)^{-1}\left(H^{T} W \mathbf{y}+\bar{W}_{k} \overline{\mathbf{x}}_{k}\right) .
$$

Here, $\hat{\mathbf{x}}_{k}$ is the best estimate of the state deviation vector in a weighted least squares sense, and is added to the nominal value of the state $\mathbf{X}^{*}$ to obtain the best estimate of the state vector $\mathbf{X}$. Eq. (3.17) is sometimes referred to as the normal equation and the $n \times n$ matrix $\left(H^{T} W H+\bar{W}_{k}\right)$ is called the information matrix. Finding the inverse of the information matrix can be difficult computationally for large values of $n$.

For the numerical simulations in this work, GEODYN orbit determination software forms the normal equations and SOLVE large linear systems solver software solves for the state deviation vector in Eq. (3.17). SOLVE utilizes Cholesky decomposition which is more accurate and stable than direct matrix inversion. The Cholesky method decomposes a symmetric positive definite matrix into a product of two triangular matrices, one lower and one upper, such that the lower triangular matrix is the transpose of the upper. Use of Cholesky decomposition thus requires that the information matrix in Eq. (3.17) be symmetric positive definite.

A full derivation and algorithm for Cholesky decomposition is presented by [ $\mathrm{Ta}$ - 
pley et al., 2004c] and a summary is given here. The normal equation of Eq. (3.17) is rewritten as:

$$
\hat{\mathbf{x}}=M^{-1} \mathbf{N} .
$$

Allowing $M$ to be a symmetric positive definite matrix and $R$ to be an upper triangular matrix such that

$$
R^{T} R=M
$$

Eq. (3.18) can be expressed as

$$
R^{T} R \hat{\mathbf{x}}=\mathbf{N}
$$

Using the definition

$$
\mathbf{z}=R \hat{\mathbf{x}}
$$

Eq. (3.20) can be written as

$$
R^{T} \mathbf{z}=\mathbf{N}
$$

where $R^{T}$ is lower triangular. The components of $\mathbf{z}$ are solved using a forward recursion relation and Eq. (3.21) is then solved using a backward recursion to determine the state deviation vector $\hat{\mathbf{x}}$.

\subsection{Gravity Recovery Simulation Procedure}

The goal of the numerical simulations is to observe how well GRACE and a potential GFO recover a realistic time-variable gravity signal. In order to assess the performance of these missions, various Truth gravity signals are used in separate simulation studies. The simulation procedure is identical regardless of which Truth signal is estimated. The end result of the numerical simulation is either a set of spherical harmonics (Section 3.4.4) or mascons (Section 3.4.5). Most of the simulations performed 
for this work apply the mascon technique, largely due to the computational advantages associated with mascon estimation as previously discussed in Section 2.4.

\subsubsection{Numerical Simulation Procedure}

A flow chart describing the numerical simulation procedure is shown in Figure 3.8 and is summarized by the following three steps:

Step 1.) Truth satellite-to-satellite range-rate data and satellite ephemeris are generated by propagating the satellites with the Truth set of force models and initial conditions as input (GEODYN run 1: Orbit propagation). Mission specific range-rate measurement noise and accelerometer noise is added to the Truth range-rate data. Errors are also added to the satellite ephemeris data. This noisy data are representative of the data which would be available from a real GRACE or GFO mission.

Step 2.) Satellite initial conditions and satellite accelerations are estimated with the Nominal set of force models and noisy range-rate and satellite position data from Step 1 (GEODYN run 2: Data reduction).

Step 3.) The observation residuals and partial derivatives are computed with the Nominal set of force models, the noisy range-rate data, the estimated satellite initial conditions from Step 2, and the estimated satellite accelerations from Step 2 (GEODYN run 3: Data reduction).

In Step 1, the GEODYN software is run in data simulation mode. The set of Truth force models used in this step represent the true Earth static and time-varying gravity signal that any orbiting satellite is sensitive to, and the simulated range-rate and satellite position data output from this GEODYN run represents perfect mission data to within numerical precision. The models, which make up the Truth force models, are given in Table 3.1. Due to limitations in available computer memory, it is necessary to break up all simulations into one-day arcs. Simulated range-rate and position observations are generated every five seconds for each one-day arc, for a total of 17,280 range-rate and position observations per arc. Throughout this work, all gravity estimates are formed 


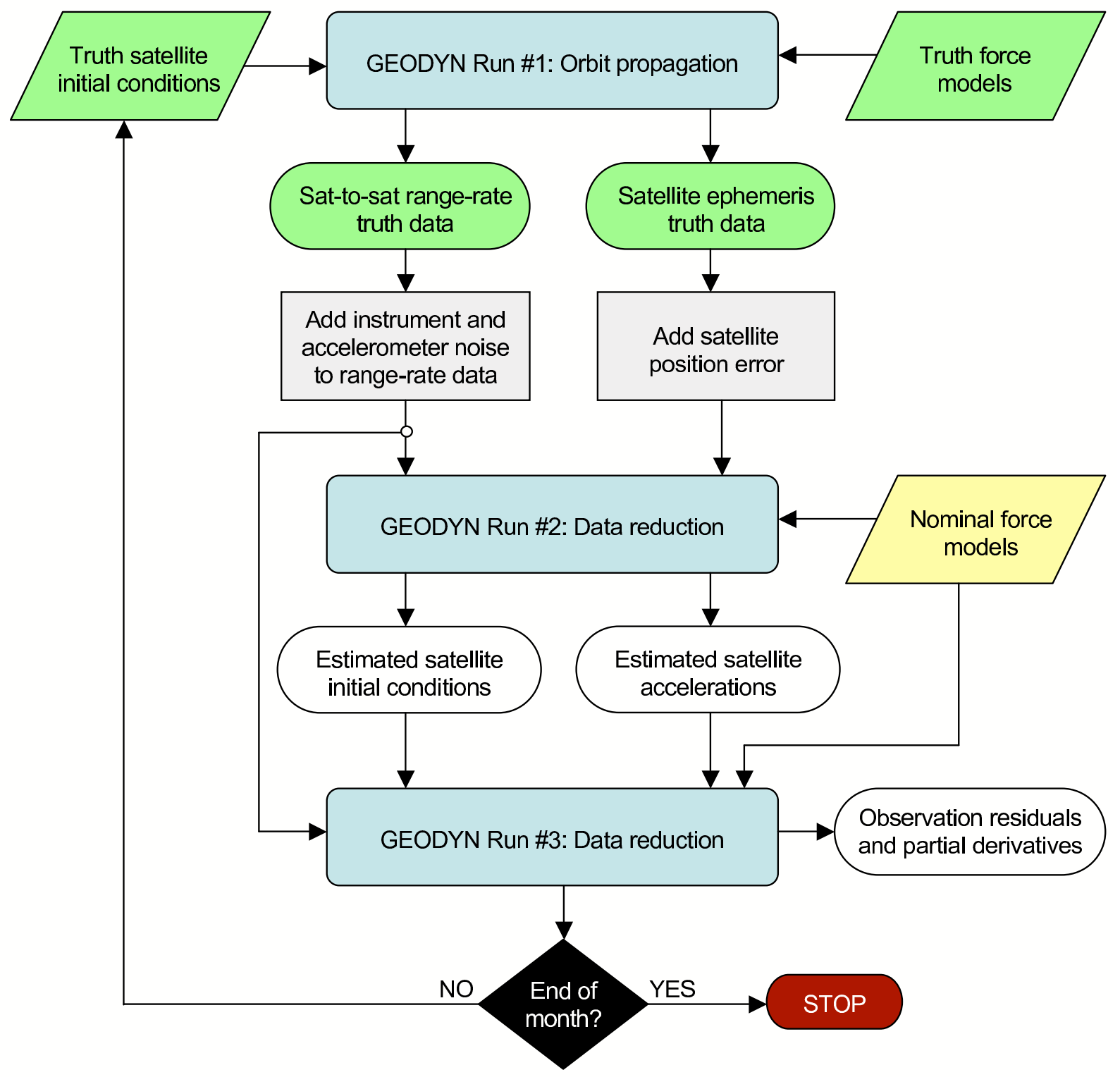

Figure 3.8: Numerical simulation procedure flow chart. 
as monthly solutions, which are formed by combining all of the the one-day arcs for an entire month into one gravity estimate. In order to introduce the appropriate level of instrument error into the numerical procedure, mission specific range-rate noise is added to the Truth range-rate data generated by the first GEODYN run. The description of the satellite-to-satellite range-rate errors of the GRACE K-band microwave ranging instrument is given in Section 4.2.1 and the errors of the interferometric laser ranging system designed for a GFO are explained in Section 4.2.2. The errors associated with the GRACE on-board accelerometer are introduced into the simulation by determining their affect on the range-rate measurements and including them as a range-rate error. This error source and the procedure for the inclusion of this error is described in Section 4.3. The resulting noisy range-rate data are then representative of real satellite-tosatellite mission data with both the range-rate and the accelerometer or GRS noise taken into account. Appropriate noise is also added to the Truth satellite ephemeris data to account for the imperfect satellite positions which are provided by GPS and is described in Section 4.4 .

In Step 2, GEODYN is run in data reduction mode, and the satellite initial conditions and satellite general accelerations are estimated. In this step the state vector $\mathbf{X}$ from Eq. (3.5) consists of twelve satellite state parameters and 384 general acceleration parameters for a total state vector length of 396. GEODYN is setup to iterate until the linear estimation procedure converges on a solution. The Nominal force models given in Table 3.1 are used in this step. The satellite states must be estimated in this intermediate step because the final simulation step, Step 3, utilizes an orbit refinement procedure where only range-rate data are used to estimate the gravity parameters and three of the twelve satellite state parameters. This procedure is discussed in Section 3.4.3. The estimation of all twelve satellite state parameters requires the satellite position data, 
which in the case of a real mission is provided by GPS tracking. The estimation of the general accelerations reduces the range-rate residuals associated with the accelerometer errors which are introduced into the data in Step 1. The simulations also show that estimating the general accelerations reduces the errors caused by temporal aliasing. This procedure which includes the accelerometer errors in Step 1, and estimates the satellite general accelerations in Step 2, is used in place of using simulated accelerometer data and estimating accelerometer parameters. It was determined that this was a reasonable way to incorporate the accelerometer errors into the numerical simulation procedure [Bettadpur, 2007; Luthcke, 2009]. A detailed description of the general accelerations estimation is given in 3.4.2. In Step 3, the estimated accelerations are fixed and the estimated satellite states are used as the initial conditions.

For Step 3, GEODYN is set up for a single iteration data reduction run, which outputs a file containing the observation residuals and partial derivatives that form the normal equation as given by Eq. (3.17). As with Step 2, the Nominal force models and the noisy range-rate data are used as inputs. The satellite state and the fixed general accelerations from Step 2 are also inputs for the final step. The state vector $\mathbf{X}$, for each arc, contains the twelve satellite initial conditions and the spherical harmonics coefficients. A $180 \times 180$ spherical harmonic expansion is described by 32,760 coefficients, so for this case the state vector has a total length of 32,772 . The twelve satellite state parameters are arc parameters, meaning they are estimated for each one-day arc. The spherical harmonic coefficients are global parameters, and are common to all of the arcs for some selected time period, which in this case is one month. At the end of Step 3, the final satellite state is used as the initial conditions for Step 1 of the next one-day arc, and the procedure is repeated until the final day of the month with each one-day run resulting in a separate partial derivative file. Once a full month of partial derivative files 
are formed they are then used to form the normal equations and determine a solution. Further detail of the spherical harmonic and mascon estimation procedures is presented in Sections 3.4.4, and 3.4.5 respectively.

The final step of the simulation procedure is to sum and then solve the normal equations for each month. This results in the gravity solution for that month. The SOLVE software package combines the normal equations from all of the one-day arcs and solves the linear system. As discussed in Section (3.3), SOLVE utilizes Cholesky decomposition to compute the inverse of the large information matrix, and determine the best estimate of the state deviation vector, $\hat{\mathbf{x}}$.

\subsubsection{General Acceleration Parameter Estimation}

The numerical simulation procedure includes the estimation of a set of general acceleration parameters for each one-day arc. The estimation of these parameters reduces the errors associated with the accelerometer which is described in Section 4.3. As that section shows, the inclusion of the accelerometer errors into the range-rate observation results in large errors, especially at the one cycle-per-revolution frequency. The RMS of the range-rate observation residuals is greatly reduced with the estimation of the general acceleration parameters and significantly improves the resulting gravity solutions.

The satellite perturbations in the radial, transverse, and normal directions can be described by:

$$
\begin{aligned}
& F_{T}=C_{T} \cos u+S_{T} \sin u+k_{T} \hat{T} \\
& F_{N}=C_{N} \cos u+S_{N} \sin u+k_{N} \hat{N} \\
& F_{R}=C_{R} \cos u+S_{R} \sin u
\end{aligned}
$$


where

$$
\begin{array}{ll}
F_{T} & =\text { transverse general acceleration } \\
F_{N} & =\text { normal general acceleration } \\
F_{R} \quad & =\text { radial general acceleration } \\
u & =\text { argument of latitude of satellite } \\
C_{T}, S_{T} & =1 \text {-cpr transverse acceleration parameter } \\
C_{N}, S_{N} & =1 \text {-cpr normal acceleration parameter } \\
C_{R}, S_{R} & =1 \text {-cpr radial acceleration parameter } \\
k_{T} & =\text { constant transverse parameter } \\
k_{N} & =\text { constant normal parameter } \\
\hat{T} & =\text { unit velocity vector } \\
\hat{N} & =\text { unit vector in normal direction }(\mathbf{r} \times \mathbf{v})
\end{array}
$$

The sum of the sin and cos terms in Eq. (3.23) describe the one cycle-per-revolution acceleration in each direction, and the $k$ terms describe the constant acceleration. A constant term, $k$, could also be estimated in the normal direction but simulations showed that its inclusion slightly degrades the gravity solutions.

Each of the above eight general acceleration parameters $(C, S$, and $k$ terms), are estimated by GEODYN once per hour, resulting in a total number of 384 estimated parameters per arc. Each parameter is assigned a correlation time, affectively reducing the number of estimated parameters, since the parameters within a given time period are forced to remain close together in value. The transverse terms are assigned a correlation time of three hours (approximately two satellite revolutions), and the radial and normal terms are assigned a correlation time of twenty-four hours. 


\subsubsection{Orbit Refinement With Baseline Parameterization}

This section summarizes the orbit refinement procedure developed by Rowlands [2002]. This method assumes that the orbit of the satellite has already been well determined with the use of GPS satellite position data. The final step in the gravity estimation procedure then involves the simultaneous estimation of the gravity field and a refined satellite state with a baseline parameterization using the satellite-to-satellite range-rate data only. In applying this method, the twelve satellite state parameters in Cartesian space are converted to a baseline parameterization, where the new state parameters are:

$$
\begin{aligned}
& P_{1} \text { - distance of baseline midpoint from the Earth's center of mass } \\
& P_{2} \text { - declination of baseline midpoint } \\
& P_{3} \text { - right ascension of baseline midpoint } \\
& P_{4} \text { - inertial } X \text { component of baseline midpoint velocity } \\
& P_{5} \text { - inertial } Y \text { component of baseline midpoint velocity } \\
& P_{6} \text { - inertial } Z \text { component of baseline midpoint velocity } \\
& P_{7} \text { - baseline vector length } \\
& P_{8} \text { - baseline vector pitch } \\
& P_{9} \text { - baseline vector yaw } \\
& P_{10} \text { - baseline rate-of-change vector pitch } \\
& P_{11} \text { - baseline rate-of-change vector pitch } \\
& P_{12} \text { - baseline rate-of-change vector yaw. }
\end{aligned}
$$

Simulations in [Rowlands et al., 2002] show that good solutions are found by estimating the parameters $P_{8}, P_{10}$ and $P_{11}$, while holding the values of the other nine parameters fixed. This method is used throughout this work and is implemented using SOLVE in the final step of the gravity estimation procedure. 
This technique provides a few advantages as compared to the simultaneous estimation of the full satellite state and the gravity field. The final gravity estimation step is less computationally intense due to the fact that only the satellite-to-satellite range-rate data are needed, whereas estimating gravity and the satellite state requries both the range-rate and GPS satellite position data. This method then takes advantage of the fact that the range-rate measurements are many orders of magnitude more accurate than the position measurements provided by GPS. Additionally, since the orbit is refined and not fully determined, it is possible to use this method in short-arc analysis, which is beneficial in a real mission scenario where there may be time periods when no data are available.

\subsubsection{Spherical Harmonic Estimation}

The normal equation of Eq. (3.17) may be directly formed by the observation partial derivative files output from Step 3 in Section 3.4.1, since the observation partials written by GEODYN are with respect to the spherical harmonic parameters. The dimension of the state vector is quite large for a high spherical harmonic expansion. A spherical harmonic expansion to degree and order 60 is described by 3,717 parameters while an expansion to degree and order 180 consists of 32,760 parameters. The number of spherical harmonic parameters is the size of $\boldsymbol{\alpha}$ in the state vector of Eq. (3.5). The value $n$ which describes the size of the $n \times n$ information matrix is the sum of the length of $\boldsymbol{\alpha}$ and the twelve satellite state parameters per arc. Due to the large size of the information matrix for a high spherical harmonic expansion, significant computing power is needed to form and solve the normal equations for each month. The length of the $m \times 1$ observation deviation vector $\mathbf{y}$ is 17,280 (the number of range-rate observations per day) multiplied by the number of one-day arcs in the month. 


\subsubsection{Mascon Parameter Estimation}

To generate a mascon solution, the partial derivatives output from Step 3 of Section 3.4.1, which make up the $H$ matrix of the normal equation, are converted to mascon space using Eq. (2.19). The normal equation is then formed and solved to determine the mascon parameter estimates. The length of the $\boldsymbol{\alpha}$ portion of the state vector is drastically reduced as compared to spherical harmonic estimation, thus significantly reducing the size of the information matrix that is inverted to form the solution. A $180 \times 180$ spherical harmonic expansion is described by 32,760 parameters while a mascon representation of the Greenland region with a similar spatial resolution $\left(1^{\circ} \times 1^{\circ}\right.$ blocks $)$ contains 2,775 parameters. This large reduction in the size of the linear system significantly reduces the computation time needed to solve the normal equation.

Throughout this work, all mascon solutions are formed using $1^{\circ} \times 1^{\circ}$ blocks. As described in Section 2.4.2 the mascon solutions are unstable without the application of constraints. The introduction of constraint matrices into the linear system, as calculated from Eq. (2.20), is mathematically equivalent to the inclusion of a a priori data weighting matrix $\bar{W}$ in the normal equation.

In the case of a simulation study, the true answer is perfectly known, so the estimates can always be compared to the truth to determine the exact level of error for each solution. For this study, the weights placed on the constraint matrices can be selected such that an optimal solution is achieved. The best-case solution is defined as the one which minimizes the RMS of the error map. Simulations show that a good starting point for the constraint matrix weight is the inverse of the average of the diagonals of the mascon portion of the information matrix (the information matrix is the inverse of the covariance matrix). Some improvement in the results is observed 
with an up-weighting of the constraints on the ocean mascons as compared to the land mascons, effectively forcing the oceans to remain close to a zero value and placing most all of the estimated signal over the land (which is where the truth signal is located). The mascon solutions formed in this study do not apply temporal constraints, meaning that the monthly solutions are not constrained to each other. Several different constraint scenarios are explored to determine the effect of the constraints on the solutions, and to select the best case setup for forming accurate mascon solutions.

Mascon solutions are formed for Greenland and South America. Table 3.2 summarizes the location information of the mascon solutions for each region.

\begin{tabular}{|l|l|l|}
\hline & Greenland & South America \\
\hline Minimum latitude & $52^{\circ}$ & $-35^{\circ}$ \\
\hline Maximum latitude & $89^{\circ}$ & $17^{\circ}$ \\
\hline Minimum longitude & $280^{\circ}$ & $274^{\circ}$ \\
\hline Maximum longitude & $355^{\circ}$ & $334^{\circ}$ \\
\hline Total number of mascons & 2775 & 3120 \\
\hline Number of land mascons & 654 & 1347 \\
\hline Number of ocean mascons & 2121 & 1773 \\
\hline
\end{tabular}

Table 3.2: Mascon definitions.

\subsection{Mission Orbital Parameters}

The final step in the simulation procedure is the selection of the mission orbital parameters. The determination of the orbital parameters will be an important part of the design for a GFO as it affects the mission performance. It is assumed here that a GFO will be placed in a near-polar, near-circular orbit as with GRACE, since these conditions are needed to provide consistent global coverage.

Of primary importance for the mission design will be the satellite altitude. Due to the attenuation of the gravity signal with increasing altitude it is desired to fly a GFO 
in as low an orbit as possible. As the altitude decreases the forcing from atmospheric drag significantly increases, increasing the required thrust capabilities to maintain the orbit or to implement a drag-free system. The selection of the spacecraft separation distance will also be an important part of the mission design. The separation distance affects the level of instrument noise as well as the ability of the mission to recover the gravity signal at various wavelengths. Lastly, the groundtrack of the mission is of great importance as global coverage is needed to generate global gravity estimates.

In this work two orbital configurations are considered. First, the current GRACE orbital parameters are used. In this configuration the satellites have an altitude of $\sim 480$ $\mathrm{km}$ and a spacecraft separation of $\sim 220 \mathrm{~km}$. Second, a possible GFO configuration is used, in which the satellites are flown at an altitude of $\sim 250 \mathrm{~km}$ with a spacecraft separation of $\sim 50 \mathrm{~km}$. Both orbits provide good global coverage every thirty days. The groundtrack spacing for thirty days is $\sim 1$ degrees for the $480 \mathrm{~km}$ altitude orbit and $\sim 0.8$ degrees for the $250 \mathrm{~km}$ altitude orbit. Table 3.3 summarizes the different orbital configurations and Figure 3.9 shows the groundtracks for the two configurations over South America and Greenland.

\begin{tabular}{|l|l|l|}
\hline & $\begin{array}{l}\text { 480 } \mathbf{~ k m} \text { altitude } \\
\mathbf{2 2 0} \mathbf{~ k m} \text { separation }\end{array}$ & $\begin{array}{l}\mathbf{2 5 0} \mathbf{~} \mathbf{~} \mathbf{5 0} \text { altitude } \\
\text { (m) }\end{array}$ \\
\hline $\mathbf{a}$ & $6860.813 \mathrm{~km}$ & $6632.876 \mathrm{~km}$ \\
\hline $\mathbf{e}$ & $1.36 \times 10^{-4}$ & $1.15 \times 10^{-5}$ \\
\hline $\mathbf{i}$ & $89.015^{\circ}$ & $89.458^{\circ}$ \\
\hline
\end{tabular}

Table 3.3: Mission orbital parameters summary. 

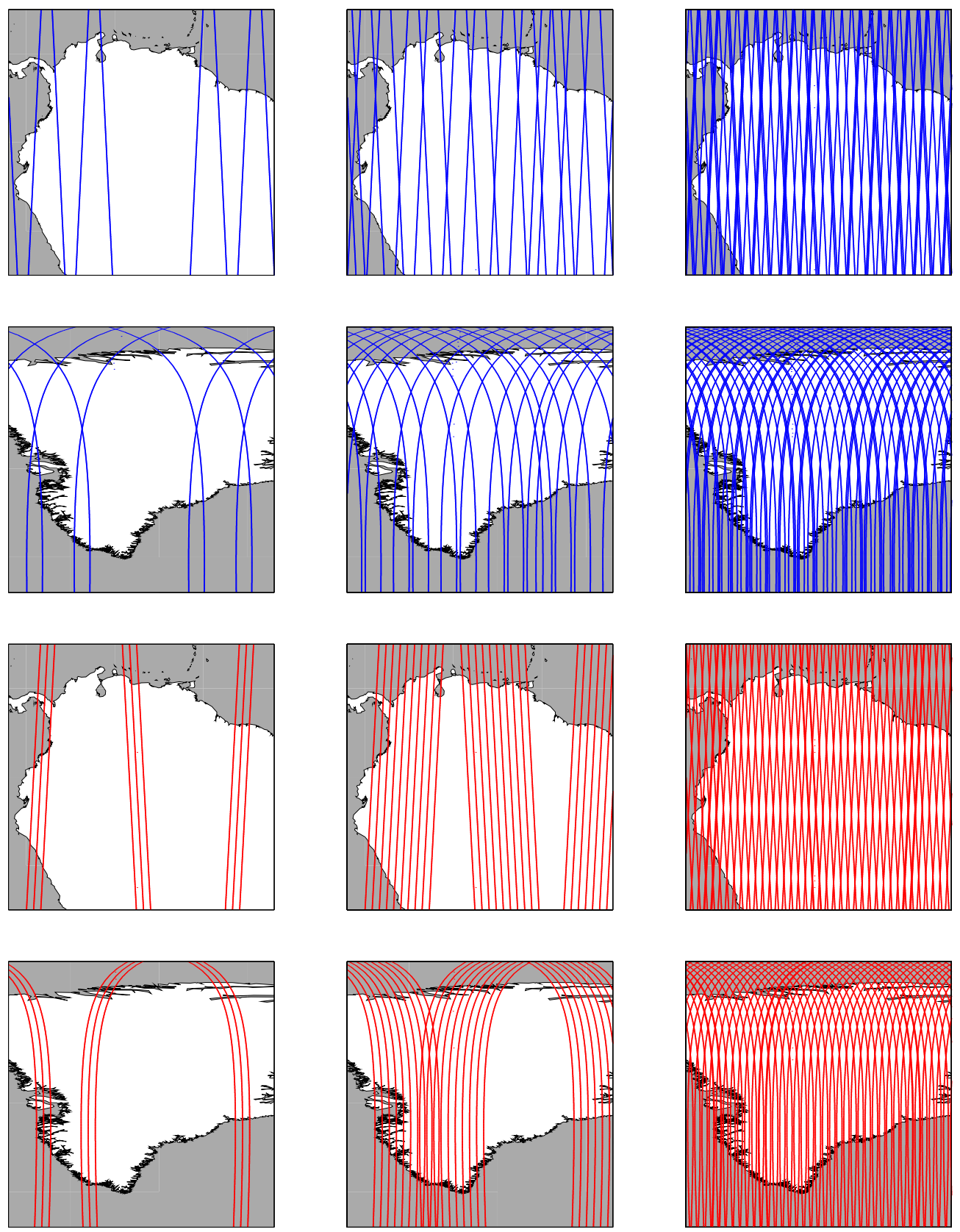

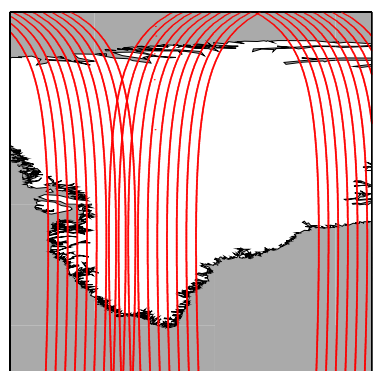

(b) 10 days

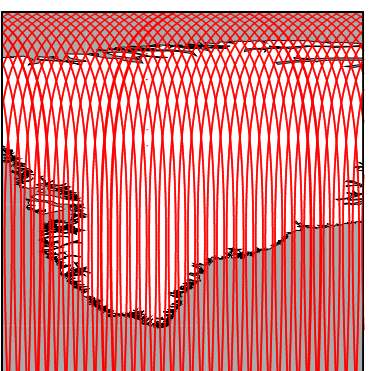

(c) 30 days

Figure 3.9: Groundtracks over South America and Greenland for the two different mission orbital parameters considered in this study. The top two rows show groundtracks for the $480 \mathrm{~km}$ altitude orbit and the bottom two rows show the $250 \mathrm{~km}$ altitude orbit. The columns show the groundtracks for (a) 3 days, (b) 10 days, and (c) 30 days. 
It is worth noting that the groundtracks attain similar coverage after 30 days, but have very different coverage when considered over shorter time spans. As the plotted groundtracks show, the lower altitude orbit fills in much closer together than the higher altitude orbit. This is due to the fact that at $\sim 250 \mathrm{~km}$ altitude, the orbit is very near to a 1-day repeating groundtrack. This groundtrack would not be well suited for estimating global gravity estimates at time scales shorter than a month due to the inhomogenous coverage over shorter times. The simulation results show that the size of the temporal aliasing errors for the regional mascon estimates is affected by the groundtrack coverage and the satellite altitude. 


\section{Chapter 4}

\section{Sources of Error}

\subsection{Introduction}

This chapter presents in detail the major error sources which must be included in the numerical simulations to achieve realistic results. The work given in this chapter comprises a major portion of this research, as computing realistic representations of all significant sources of error is a difficult task, and requires a great attention to detail. The sources of error presented in order are: the inter-satellite measurement errors, the accelerometer errors, the satellite positioning errors, and the temporal aliasing errors. The chapter concludes with a summary of the GRACE and GRACE follow-on mission configurations, which are defined by a combination of the selected satellite orbital parameters from Section 3.5, and the appropriate error sources as determined by the selected on-board instruments as described in this chapter.

\subsection{Inter-satellite Measurement Errors}

A main goal of this work is to investigate the effects of an improved ranging device on the performance of a GRACE Follow-On mission. It is expected that a GFO with more accurate ranging measurements would improve the spatial resolution of the gravity estimates. 
System noise is often described in terms of a power spectral density (PSD), or the amplitude spectral density (ASD) where the ASD is equal to the square-root of the PSD (from this point forward the $\mathrm{PSD}^{1 / 2}$ will simply be referred to as the spectral density). The spectral density plots describing the noise for GRACE and GFO are presented in this section. For more detail on spectral densities refer to Appendix C.

\subsubsection{GRACE}

The spectral density of the satellite-to-satellite ranging measurement noise is given by $1.8 \mu \mathrm{m} / \sqrt{\mathrm{Hz}}[$ Bettadpur, 2007]. The numerical simulations in this work use satelliteto-satellite range-rate tracking data so the spectral density must be converted from range to range-rate. Differentiation in the time domain is equivalent to multiplication of $2 \pi f$ in the frequency domain. The spectral density for the range-rate error is then given by $1.8 \cdot 2 \pi f \mu m / s / \sqrt{H z}$. The error budgets for GRACE in terms of range and range-rate are shown in Figure 4.1.

The instrument error is considered over frequencies of $10^{-4} \mathrm{~Hz}$ to $10^{-1} \mathrm{~Hz}$. The highest frequency which can be sampled is called the Nyquist frequency and is equal to $1 /(2 \Delta t)$ where $\Delta t$ is the sampling rate. For the simulations in this study a sampling rate of 5 seconds is used, making the Nyquist frequency equal to $10^{-1} \mathrm{~Hz}$.

In order to include the satellite-to-satellite tracking error into the numerical simulations the spectral density is converted into a time series of range-rate errors by taking the inverse Fourier transform of the range-rate spectral density as given by Eq. (B.4). Refer to Appendix B for a detailed explanation of the procedure. The resulting RMS of the simulated GRACE range-rate errors is $\sim 0.21 \mu \mathrm{m} / \mathrm{s}$. 


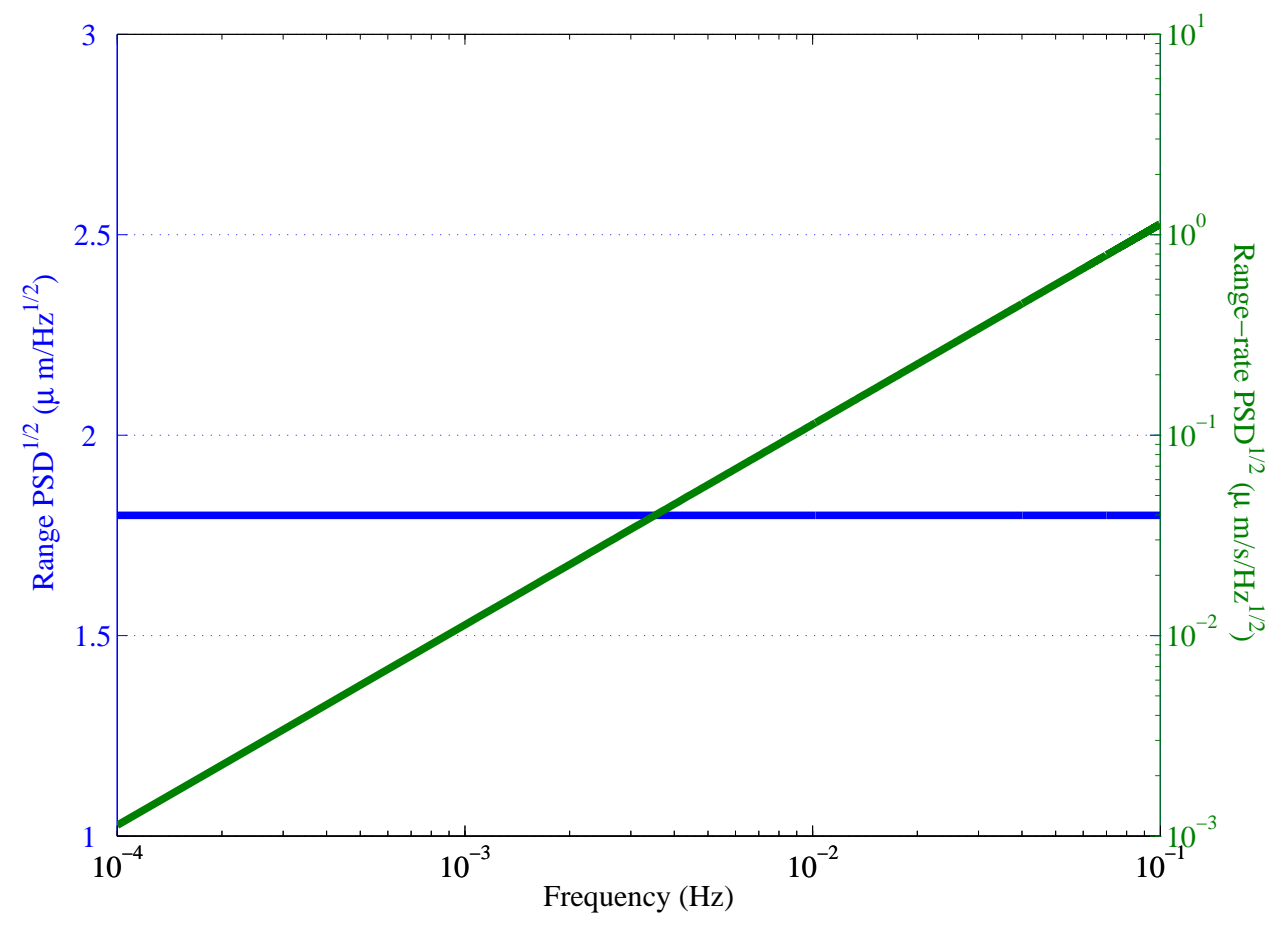

Figure 4.1: Spectral densities for GRACE satellite-to-satellite measurement error. The blue line is range and the green line is range-rate.

\subsubsection{GRACE Follow-On}

Detailed mathematical representations of the various errors sources which would affect the GRACE Follow-On satellite-to-satellite measurement errors are available for this study. The spectral density of errors for the interferometric laser ranging system developed by Ball Aerospace \& Technologies Corp. are detailed in [Pierce et al., 2008]. Most of the components of the error budget were tested and verified by measuring the actual errors on a brassboard model in the laboratory. The error budget is shown in Figure 4.2 .

As before, the spectral density in terms of range-rate is found by multiplying each term by $2 \pi f$. A time series of range-rate errors is then found for each error source. The 


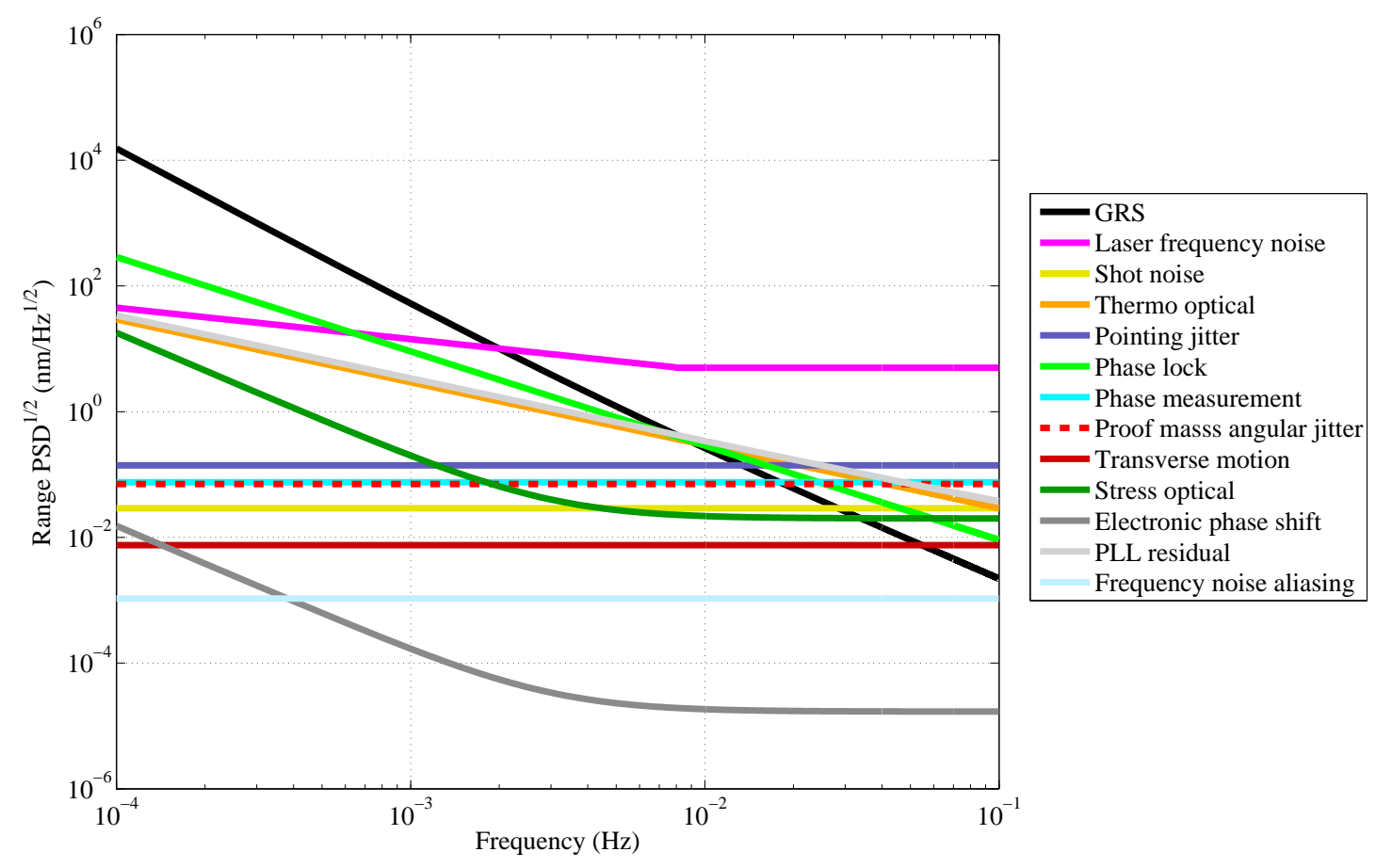

Figure 4.2: Spectral density for interferometric laser ranging system with a spacecraft separation distance of $50 \mathrm{~km}$.

resulting time series are then summed to generate the total range-rate error time series. The RMS of the simulated GFO range-rate errors is $\sim 0.58 \mathrm{~nm} / \mathrm{s}$

\subsection{Accelerometer Errors}

Each of the GRACE satellites is equipped with an accelerometer. The accelerometers measure the electrostatic force required to hold the position of a proof mass constant with respect to the satellite, where the proof mass is located at the center-of-mass of the spacecraft. The satellite and the proof mass are affected by the same gravitational forces but the proof mass is not affected by the non-gravitational forces which act on the outside of the satellite. The accelerometer data are then used to differentiate between the gravitational and non-gravitational forces which act on each satellite, meaning that 
errors in the accelerometer measurements corrupt the GRACE gravity estimates and must be considered.

For this work, the accelerometer error is included in the numerical simulations by calculating its effect on the range-rate error. The mathematical procedure which determines the accelerometer error is given in the appendices. A simplified equation which describes the effect of perturbed motion on the range-rate is developed in Appendix D. This equation is then applied to Hill's equations in Appendix E to determine the spectral density function that describes the accelerometer error effect on the range-rate errors.

Table 4.1 gives the spectral density of the accelerometer errors for the three satellite axes. The normal satellite direction was chosen as the least sensitive accelerometer axis for GRACE because errors in the normal direction have the smallest affect on the satellite-to-satellite range-rate measurements [Kim, 2000].

\begin{tabular}{|l|l|}
\hline Direction & PSD $^{1 / 2}$ \\
\hline Radial & $(1+0.005 / f)^{1 / 2} \times 10^{-10} m / s^{2} / H z^{1 / 2}$ \\
\hline Transverse & $(1+0.005 / f)^{1 / 2} \times 10^{-10} m / s^{2} / H z^{1 / 2}$ \\
\hline Normal & $(1+0.1 / f)^{1 / 2} \times 10^{-9} m / s^{2} / H z^{1 / 2}$ \\
\hline
\end{tabular}

Table 4.1: Spectral density of accelerometer errors for satellite axes.

Figure 4.3 shows the spectral density of the range-rate error due to errors in the accelerometer as calculated by Eq. (E.6). A strong spike in the spectral density function is present at the one cycle-per-revolution frequency. As in the previous section, the time series of range-rate error is found by taking the inverse Fourier transform of the spectral density. This resulting time series is then added to the satellite-to-satellite range-rate error time series from Section 4.2 to form the final time series of range-rate errors as plotted in Figure 4.4. The combined error time series is clearly dominated by errors at 
the one cycle-per-revolution frequency and has an RMS of $\sim 1.29 \mu \mathrm{m} / \mathrm{s}$.

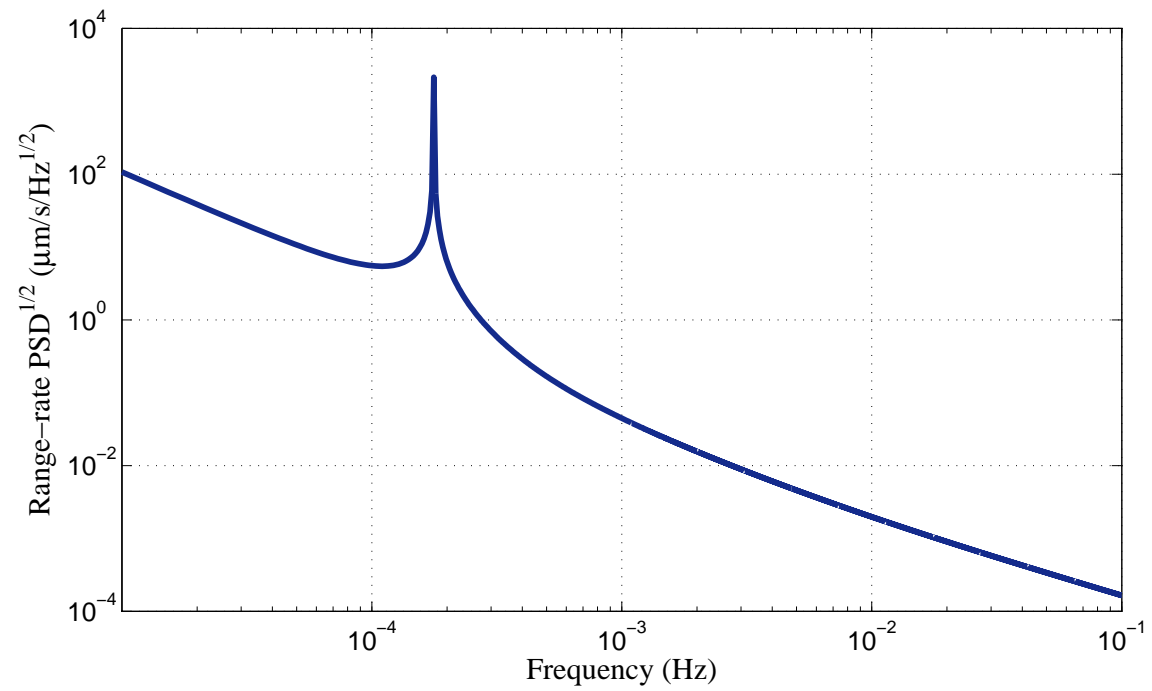

Figure 4.3: Spectral density of range-rate error due to accelerometer error.

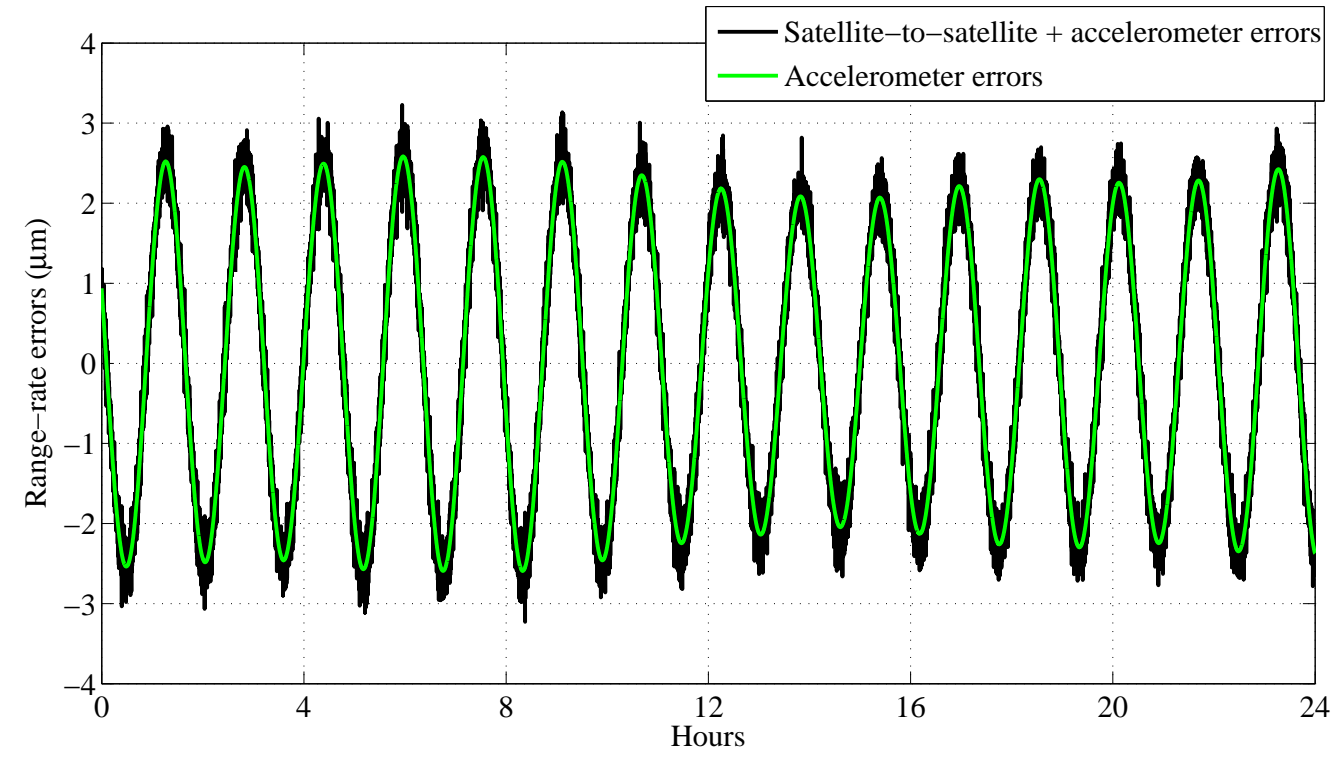

Figure 4.4: Time series of range-rate errors. 
The errors associated with the gravitational reference sensor are already included in the error budget for the laser ranging system described in Section 4.2.2, so the process described in this section does not apply to the drag-free simulation cases.

\subsection{Satellite Positioning Errors}

The gravity estimation procedure requires that the satellite orbits are accurately determined. For GRACE, the orbits are usually determined using a dynamic orbit determination method which combines GPS tracking data with orbital dynamics. This method seeks to optimally weight the geometric (GPS) information with the dynamic (force models) information to simultaneously estimate the orbit and adjust the force models. The orbit accuracy is then dependent on errors in the GPS measurements and the force models used in the procedure.

The accuracy of the final orbits is not central to this study, but the GPS measurement error must be taken into account for the numerical simulations since errors in the satellite position degrade the gravity solutions. According to Kang [2006] the GPS tracking data for GRACE orbit determination has a precision of $\sim 1.0 \mathrm{~cm}$.

The effect of the GPS tracking errors is introduced into the simulations by adding

a Gaussian white noise with $\sigma=1.0 \mathrm{~cm}$ to the simulated truth position vectors in all three axis directions. The resultant time series of satellite position errors has an RMS of $\sim 1.74 \mathrm{~cm}$, which is slightly pessimistic as compared to the results of Kang [2006]. A one-day time series and the corresponding histogram of the satellite position errors are shown in Figure 4.5. 

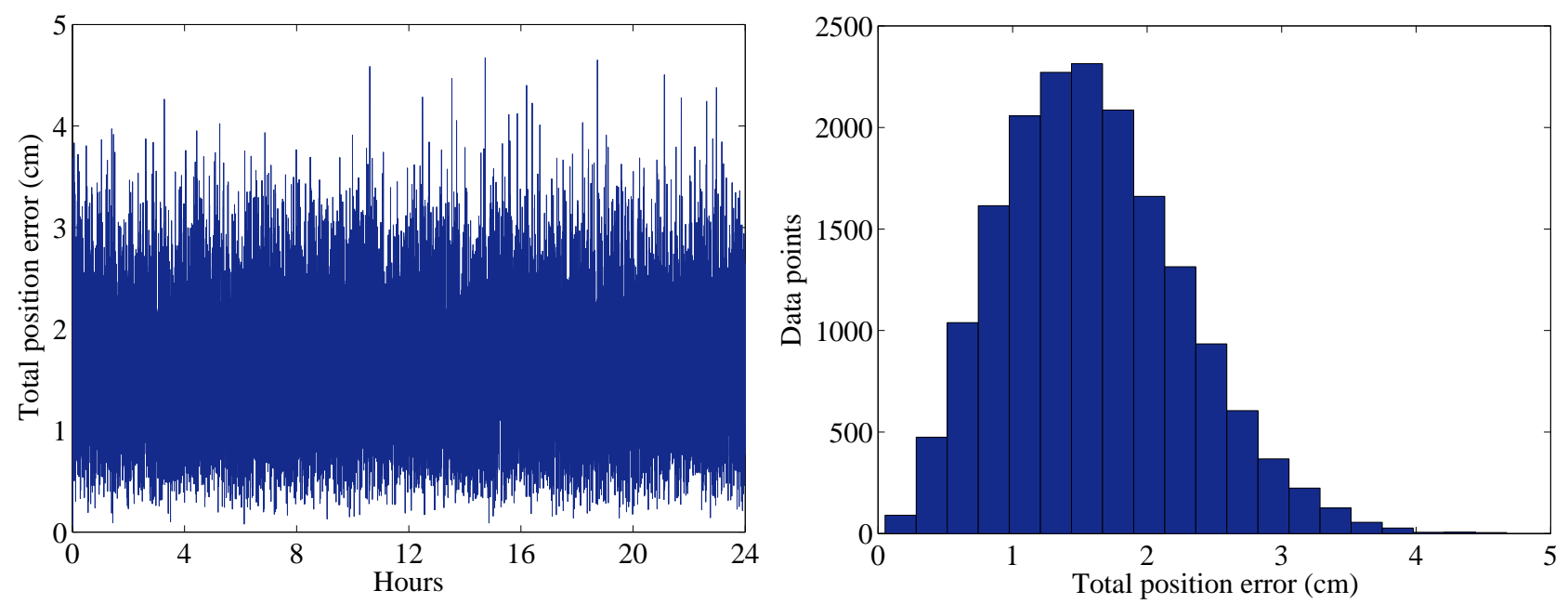

Figure 4.5: One-day time series and histogram of satellite position errors. RMS $=$ $\sim 1.74 \mathrm{~cm}$.

\subsection{Temporal Aliasing Errors}

Temporal aliasing errors exist when a sampled signal has a higher frequency than the rate at which it is sampled. For GRACE, aliasing errors exist because water mass changes from atmospheric, oceanographic, tidal, and hydrologic signals occur at time scales of a few hours, while GRACE gravity solutions are usually formed about once per month. A number of studies have shown that the problem of temporal aliasing is a major source of error for GRACE [Encarnacao et al., 2009; Moore and King, 2008; Seo et al., 2008b,a; Ray and Luthcke, 2006; Han et al., 2004; Thompson et al., 2004]. This error is introduced into the simulations by using a different set of Truth and Nominal models as explained in Section 3.2.

The work of Seo et al. [2008a] identifies two different types of temporal aliasing errors. Aliasing caused by mis-modeling of the oceanic and atmospheric signals produces large errors in the resonant spherical harmonic orders as described by Kaula [2000]. For the GRACE groundtrack this occurs around spherical harmonic order multiples of 15 . 
This type of aliasing is also largely responsible for the correlated pattern that exists between even and odd degrees for a given order. This correlation was observed by Swenson and Wahr [2006b], and causes north-south striping in the GRACE gravity solutions. The second type of observed aliasing is caused by tide model error. This type is mostly present in the low degree spherical harmonic terms and has long aliasing periods.

The problem of temporal aliasing is illustrated by Figure 4.6. The black line represents the high-frequency mass changes and the blue dots are the times at which the signal is sampled. The dashed black line is the true mean of the signal and the dashed blue line shows the estimated mean, as determined by the data samples. There is a clear difference between the true mean and the estimated mean caused by the under sampling of the data. Here the black line could represent the errors in the AOD and tide models used to remove unwanted signal, or the time-variable signal to be estimated (e.g. GLDAS).

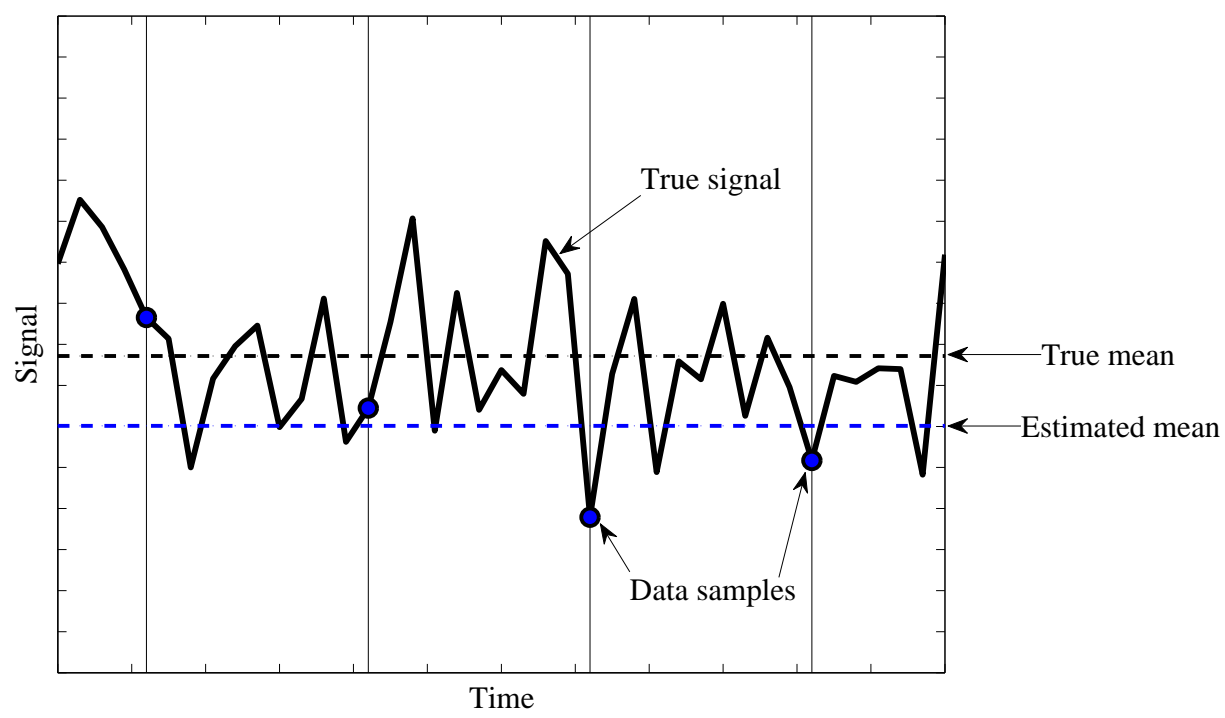

Figure 4.6: Illustration of temporal aliasing error. 
Errors in the atmospheric, oceanic, and tidal models have a spatial dependence due to limitations in the availability and quality of the data that is used to form the models. This non-uniform character of the model errors causes the temporal aliasing errors to be dependent on the satellite groundtrack, and the location for which the gravity signal is estimated. The spatial characteristics of the AOD model errors are presented in Figure 4.7. The plot shows the spatial RMS of the difference between the Truth ECMWF/OMCT and the Nominal NCEP/MOG2D AOD models for twelve monthly means. Figure 4.8 shows the same information as Figure 4.7, except that the plots are focused on South America and Greenland. Since the AOD model errors are larger over Greenland, the temporal aliasing errors caused by imperfections in the AOD models will be greater for Greenland than South America.

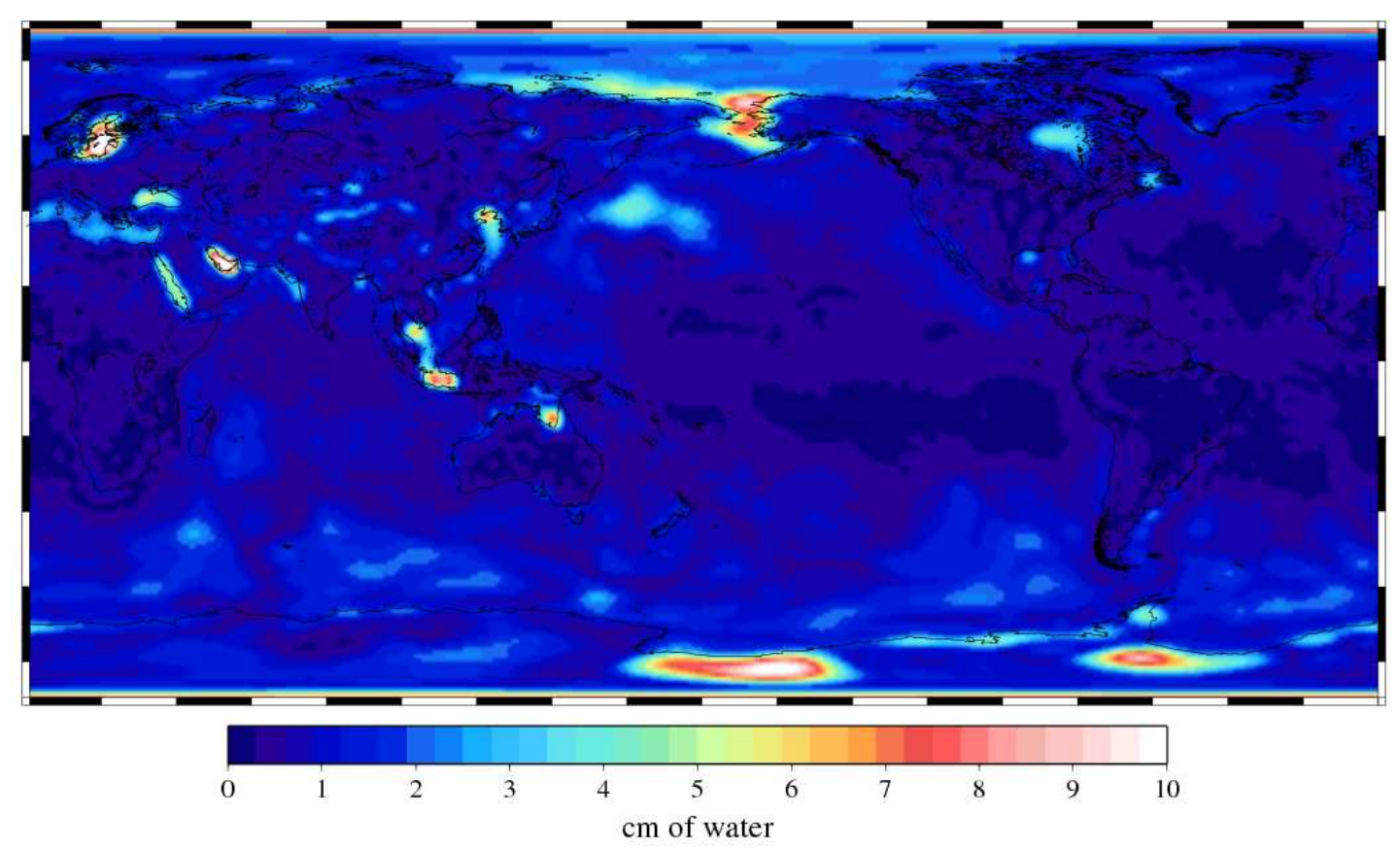

Figure 4.7: Global AOD model error RMS about the mean calculated with twelve monthly means (ECMWF/OMCT minus NCEP/MOG2D). 

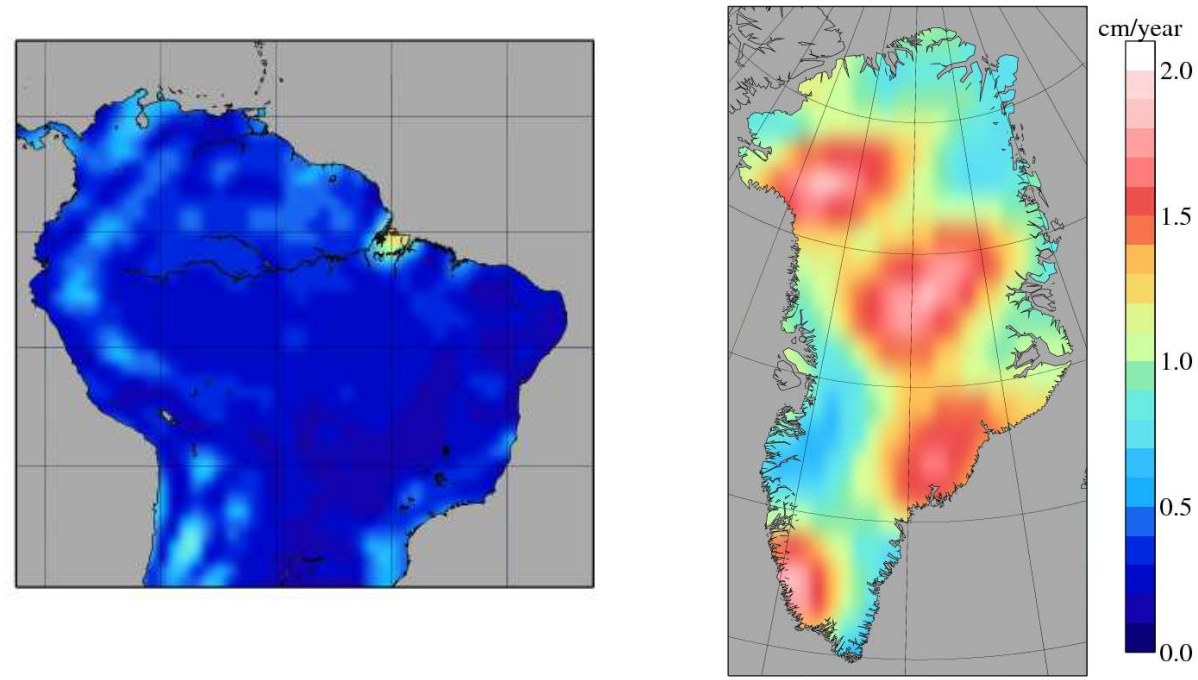

Figure 4.8: South America and Greenland AOD model error RMS about the mean calculated with twelve monthly means. (ECMWF/OMCT minus NCEP/MOG2D).

\subsection{Summary of GRACE and GFO Mission Configurations}

The numerical simulations are performed for four different mission configurations and are summarized in Table 4.2.

\begin{tabular}{|l|l|l|l|}
\hline Mission & $\begin{array}{l}\text { Altitude / } \\
\text { satellite separation }\end{array}$ & $\begin{array}{l}\text { Range-rate } \\
\text { noise }\end{array}$ & $\begin{array}{l}\text { Removal of non- } \\
\text { conservative forces }\end{array}$ \\
\hline GRACE & $480 \mathrm{~km} / 220 \mathrm{~km}$ & K-band & On-board accelerometer \\
\hline GFO Case 1 & $480 \mathrm{~km} / 220 \mathrm{~km}$ & Laser & On-board accelerometer \\
\hline GFO Case 2 & $250 \mathrm{~km} / 50 \mathrm{~km}$ & K-band & GRS (drag-free) \\
\hline GFO Case 3 & $250 \mathrm{~km} / 50 \mathrm{~km}$ & Laser & GRS (drag-free) \\
\hline
\end{tabular}

Table 4.2: Summary of gravity mission configurations.

Simulating the performance of GRACE and three different GRACE Follow-On missions will determine the effects of the various error sources on the gravity solutions. Clearly, GFO Case 3 is a best-case scenario for a GRACE Follow-on gravity mission, as it flies in the lower altitude, and reduces both the range-rate and accelerometer errors. This mission would be more expensive to fly than the other considered GFO 
missions, so it is unlikely that a GFO Case 3 mission would be realized in the near future. GFO Case 1 and GFO Case 2 are essentially hybrid missions, which combine new and heritage technologies. The simulation results should inform whether the budget of a future gravity mission would be better spent on a lower altitude combined with a drag-free system or on a laser ranging system. 


\section{Chapter 5}

\section{Simulation Results and Error Analysis}

\section{$5.1 \quad$ Introduction}

This chapter contains the results of all numerical simulations. In order to maintain consistency, prior to analyzing the gravity estimates, all of the mascon solutions are converted to a set of spherical harmonics. This is necessary to perform the best comparison between the input truth signal and the estimates since the truth signal is defined by a set of spherical harmonics.

As is the convention for GRACE gravity data, results are given as a time series of spatial maps and as a time series of mass changes for a given location. Estimates of the trend, amplitude of the annual signal, and phase are calculated from the mass change time series. For a simulation study the truth is perfectly known, so the estimates may be directly compared to the truth to determine the exact level of error. Several modes of comparison are explained in Section 2.6, and are used throughout this chapter. Simulation results are presented for four different mission configurations: GRACE, and GFO cases 1, 2, and 3 which are summarized in Section 4.6.

The Truth gravity plots to which the results of this chapter are compared are shown in Figure 3.4 and Figure 3.7. 


\subsection{Basin Definitions}

As previously discussed in Section 1.2, GRACE data are often used to calculate mass variations within some defined region, and has specifically been used to monitor water mass changes for different basins in South America and Greenland. The same principles are applied to the simulated gravity estimates to determine the ability of each mission configuration to monitor the mass change signal for a number of different basins that are defined below.

For a mascon solution, the total mass within a given region is determined by simply summing the mascons within the region. For a spherical harmonic solution the total mass inside a region is calculated with an averaging kernel (see Section 2.5), and is used in this study to measure the mass changes within each basin for spherical harmonic solutions. Swenson and Wahr [2002] discuss techniques that minimize the error when using averaging kernels. Since the true solution is known, the averaging kernels for each basin can be optimized by adjusting the parameters that define the kernel, so that the estimates most closely match the solution. The design parameters for the averaging kernel are the variable $\vartheta(\phi, \lambda)$ in Eq. (2.21), which defines the location of the exact averaging kernel, and $r_{1 / 2}$ from Eq. (2.32), which defines the level of Gaussian smoothing applied to the kernel.

It is sometimes a good idea to expand the size of $\vartheta(\phi, \lambda)$, so that the transition from a value of one inside the basin to zero outside the basin, occurs mostly outside of the basin. This is especially important for averaging kernels for Greenland as the majority of the signal is located near the coast. Three different averaging kernels are tested on the spherical harmonic results of Section 5.7 to determine the best possible kernel for recovering the mass within each basin. These are: 1.) the exact basin, 2.) 
the exact basin expanded by one degree blocks, and 3.) the exact basin expanded by two degree blocks. The values of $r_{1 / 2}$ are simultaneously varied to determine the optimal averaging kernel for each basin. For the Truth spherical harmonics, and the mascon estimates converted to harmonics, the exact averaging kernel for the exact basin location is used to determine all basin mass values.

\subsubsection{South America}

The South American basin definitions used in this study are taken from data at <http://www.r-hydronet.sr.unh.edu/grids/networks/sa.html > and are shown in Figure 5.1 along with the corresponding surface areas for each basin.

\subsubsection{Greenland}

The basin definitions for Greenland are approximately the same as those used by Luthcke et al. [2006]. Basins 1 through 6 have an elevation below $2000 \mathrm{~m}$, while basins 7 through 12 have an elevation above $2000 \mathrm{~m}$. The $2000 \mathrm{~m}$ elevation cutoff corresponds to the locations in the constructed Truth signal that define the amplitude of the annual signal (see Figure 3.6). Figure 5.2 shows the locations and surface areas of the basins. 


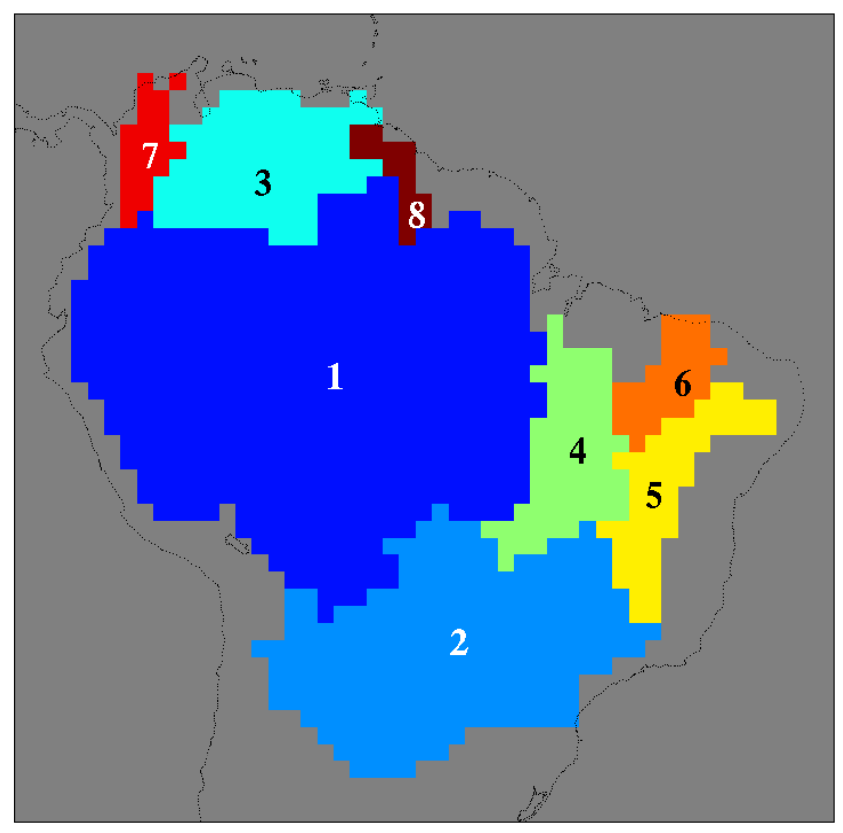

\begin{tabular}{|l|c|c|}
\hline $\begin{array}{l}\text { Basin } \\
\text { number }\end{array}$ & $\begin{array}{c}\text { Surface } \\
\text { Area }\left(\mathrm{km}^{2}\right)\end{array}$ & $\begin{array}{c}\text { Number } \\
\text { of mascons }\end{array}$ \\
\hline 1 (Amazon) & $6.0856 \times 10^{6}$ & 497 \\
\hline 2 (Parana) & $2.5516 \times 10^{6}$ & 223 \\
\hline 3 (Orinoco) & $1.0720 \times 10^{6}$ & 87 \\
\hline 4 (Tocantins) & $7.7670 \times 10^{5}$ & 64 \\
\hline 5 & $6.1403 \times 10^{5}$ & 51 \\
\hline 6 & $3.5660 \times 10^{5}$ & 29 \\
\hline 7 & $2.5826 \times 10^{5}$ & 21 \\
\hline 8 & $1.7273 \times 10^{5}$ & 14 \\
\hline
\end{tabular}

Figure 5.1: Location and size of South America basins. 


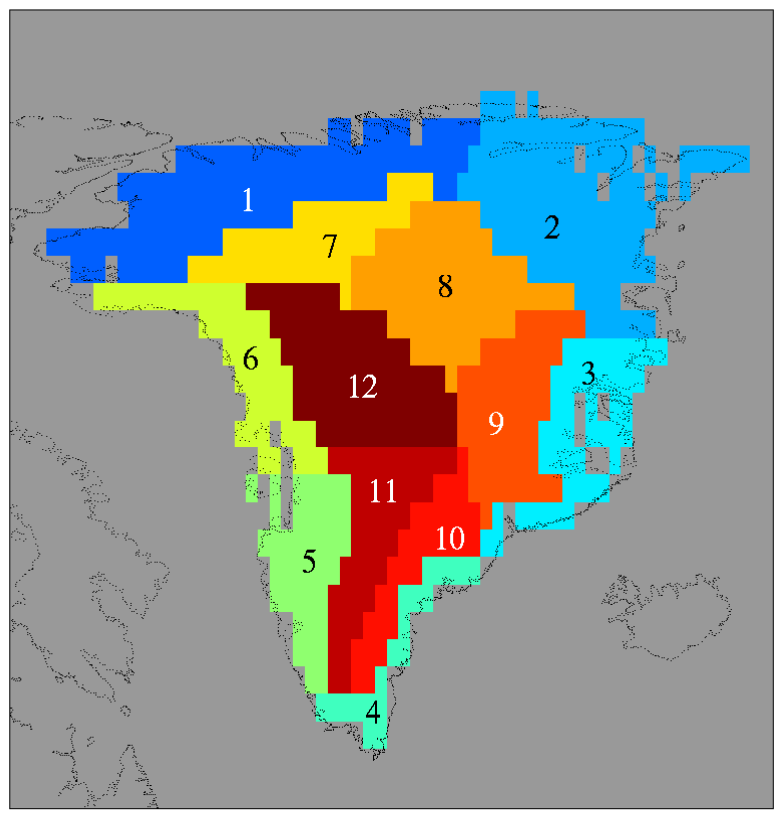

\begin{tabular}{|l|c|c|}
\hline $\begin{array}{l}\text { Basin } \\
\text { number }\end{array}$ & $\begin{array}{c}\text { Surface } \\
\text { Area }\left(\mathrm{km}^{2}\right)\end{array}$ & $\begin{array}{c}\text { Number of } \\
\text { mascons }\end{array}$ \\
\hline 1 & $2.0752 \times 10^{5}$ & 99 \\
\hline 2 & $2.2534 \times 10^{5}$ & 104 \\
\hline 3 & $1.7889 \times 10^{5}$ & 46 \\
\hline 4 & $1.0992 \times 10^{5}$ & 20 \\
\hline 5 & $1.9494 \times 10^{5}$ & 40 \\
\hline 6 & $1.4956 \times 10^{5}$ & 44 \\
\hline 7 & $1.0332 \times 10^{5}$ & 42 \\
\hline 8 & $1.9097 \times 10^{5}$ & 68 \\
\hline 9 & $1.9137 \times 10^{5}$ & 51 \\
\hline 10 & $1.4674 \times 10^{5}$ & 30 \\
\hline 11 & $1.9638 \times 10^{5}$ & 42 \\
\hline 12 & $2.3570 \times 10^{5}$ & 68 \\
\hline
\end{tabular}

Figure 5.2: Location and size of Greenland basins. 


\subsection{Mascon Estimation Methods}

The mascon gravity estimation technique allows for more flexibility than spherical harmonic estimation. The mascons can be constructed into any size and shape, and the constraint equations written between mascons can be set up to reflect the expected correlation in their signals. The results in this section show the effect of different constraint scenarios on the solutions. The method which yields the most accurate results is then used to determine the best case solutions for the four mission configurations in Sections 5.4 and 5.5. Simulations in this section are for GFO Case 3 where all error sources are included. 


\subsection{1 $\quad 1^{\circ} \times 1^{\circ}$ Mascon Grid: One Constraint Matrix}

The results for the one constraint matrix scenario are presented here. For this case all of the mascons are constrained to each other. Figure 5.3 shows the spectral density of the monthly spatial maps, their mean, and the mean of the Truth. The power of the estimate is underestimated at lower degrees due to the applied constraints, and the fact that the ocean and land mascons are constrained to each other.

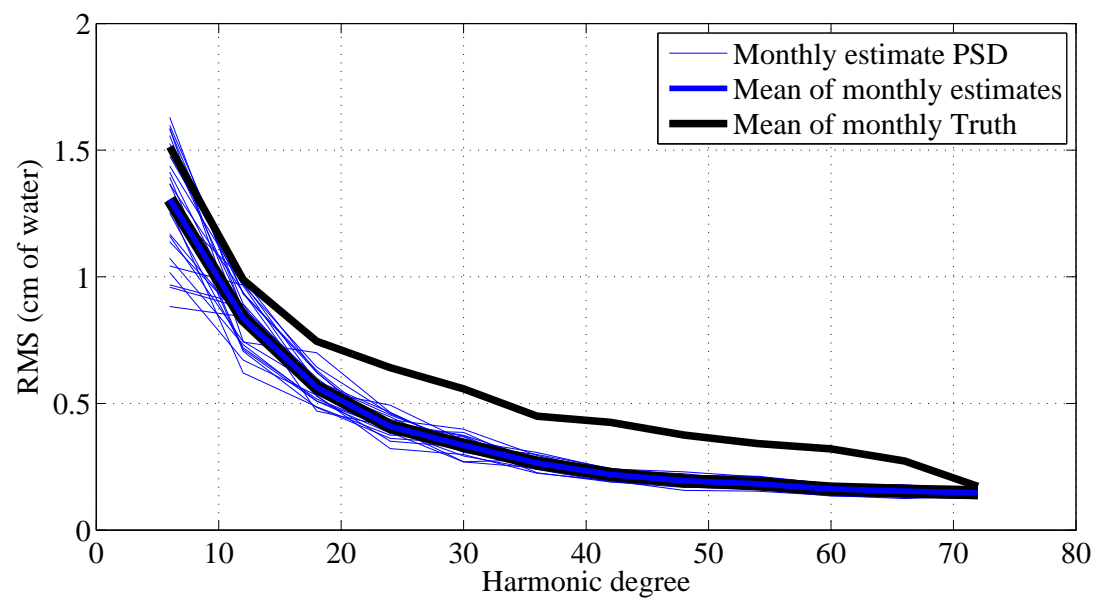

(a) South America

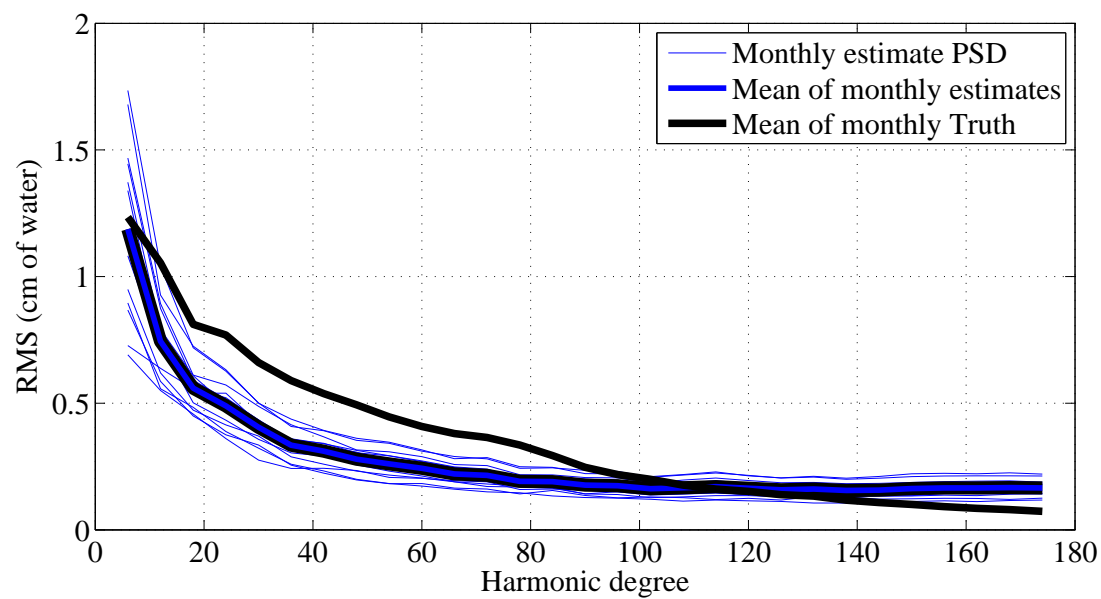

(b) Greenland

Figure 5.3: Spectral density of spatial maps in (a) Figure 5.4 and (b) Figure 5.5. 

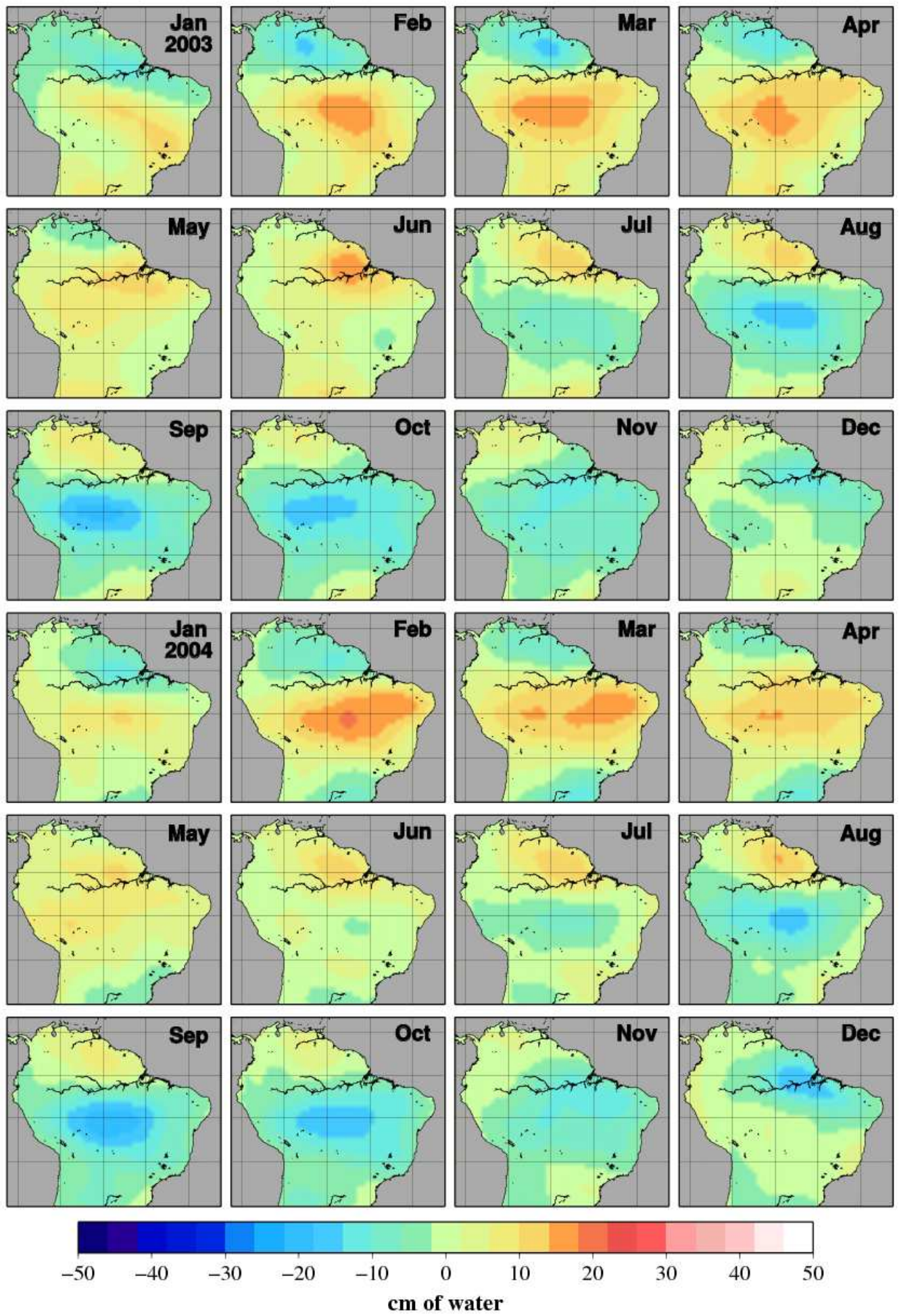

Figure 5.4: South America gravity estimates for one constraint matrix. 

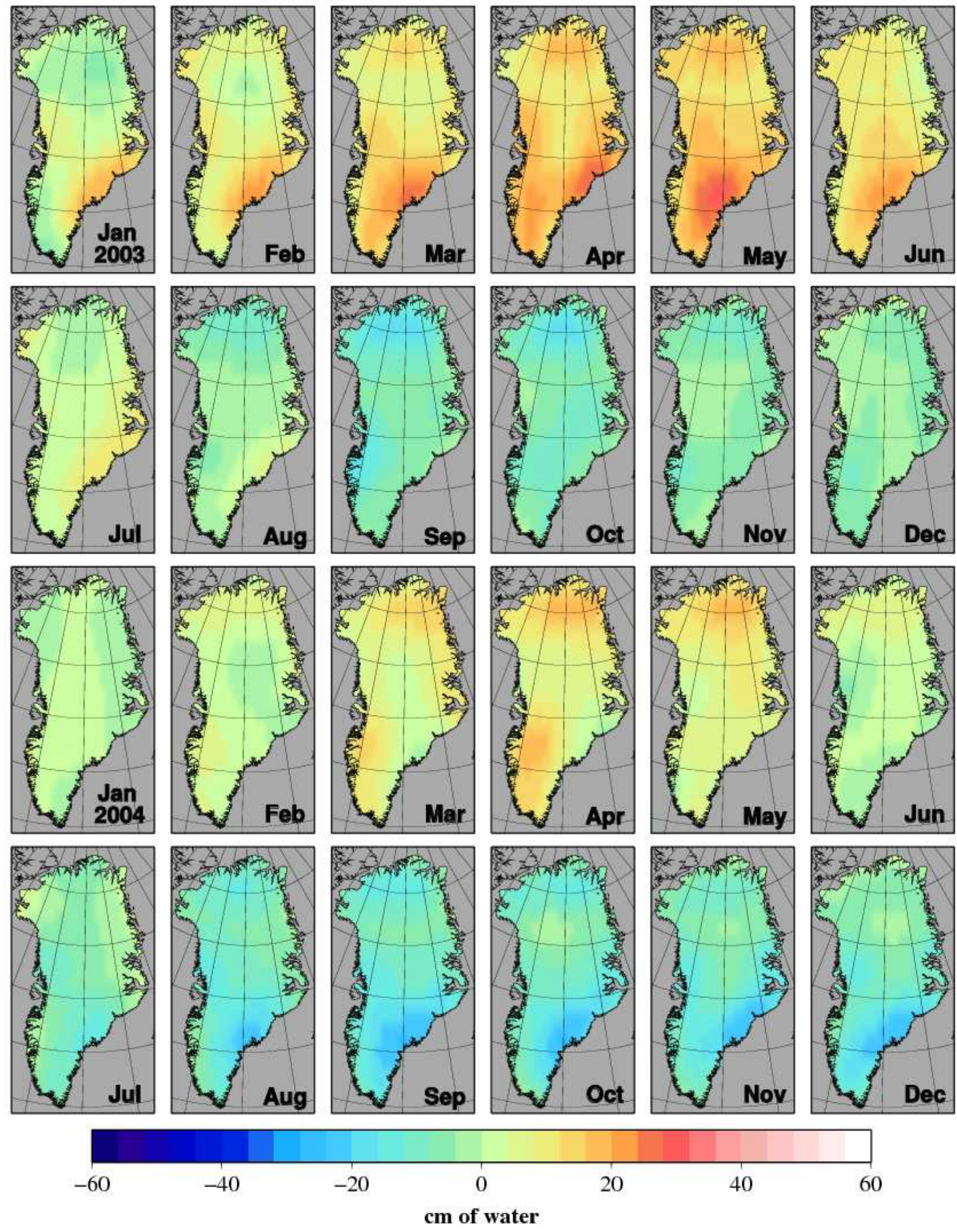

Figure 5.5: Greenland gravity estimates for one constraint matrix. 


\subsection{2 $1^{\circ} \times 1^{\circ}$ Mascon Grid: Separate Land and Ocean Constraints}

The scenario now presented applies separate constraints to the land and ocean mascons, and is implemented by constructing two separate constraint matrices. Once again, the power of the signal is underestimated at lower degrees, but not as much as it was for the previous case. The weight of the constraints could be adjusted to improve the power of the estimate, but this results in a higher RMS of the error map.

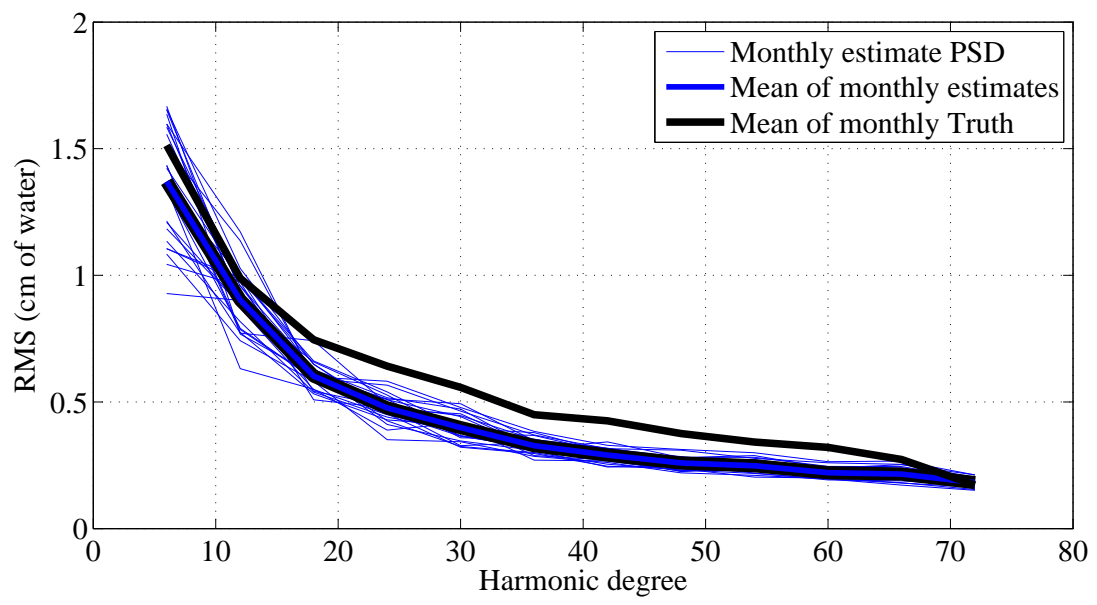

(a) South America

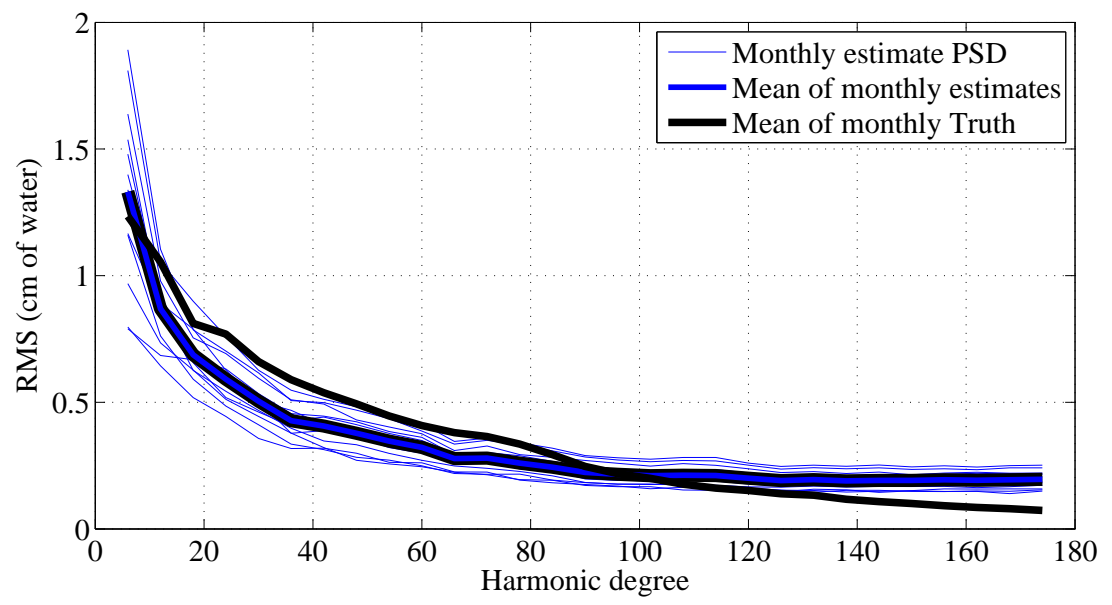

(b) Greenland

Figure 5.6: Spectral density of spatial maps in (a) Figure 5.7 and (b) Figure 5.8. 

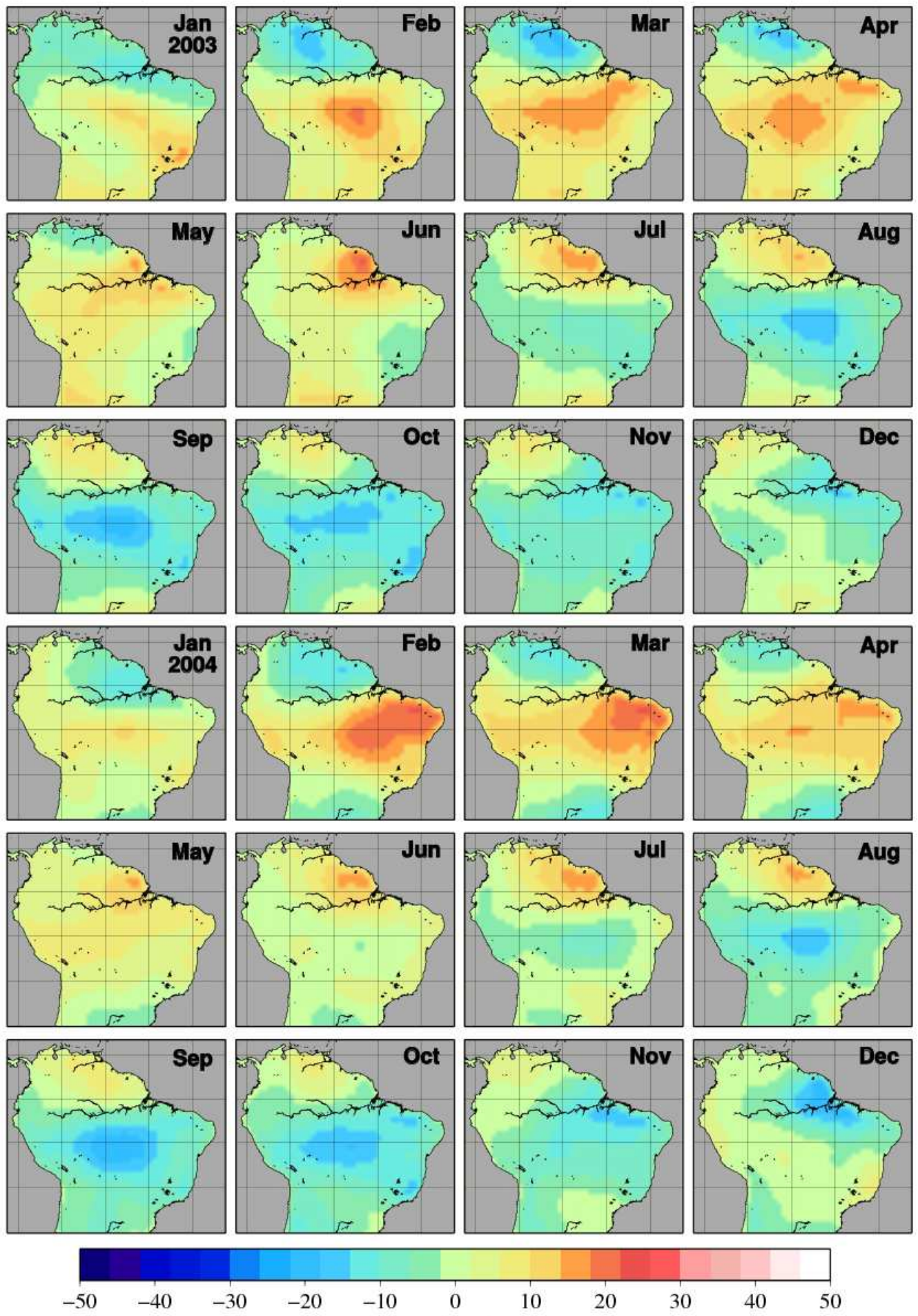

cm of water

Figure 5.7: South America gravity estimates for separate land and ocean constraints. 

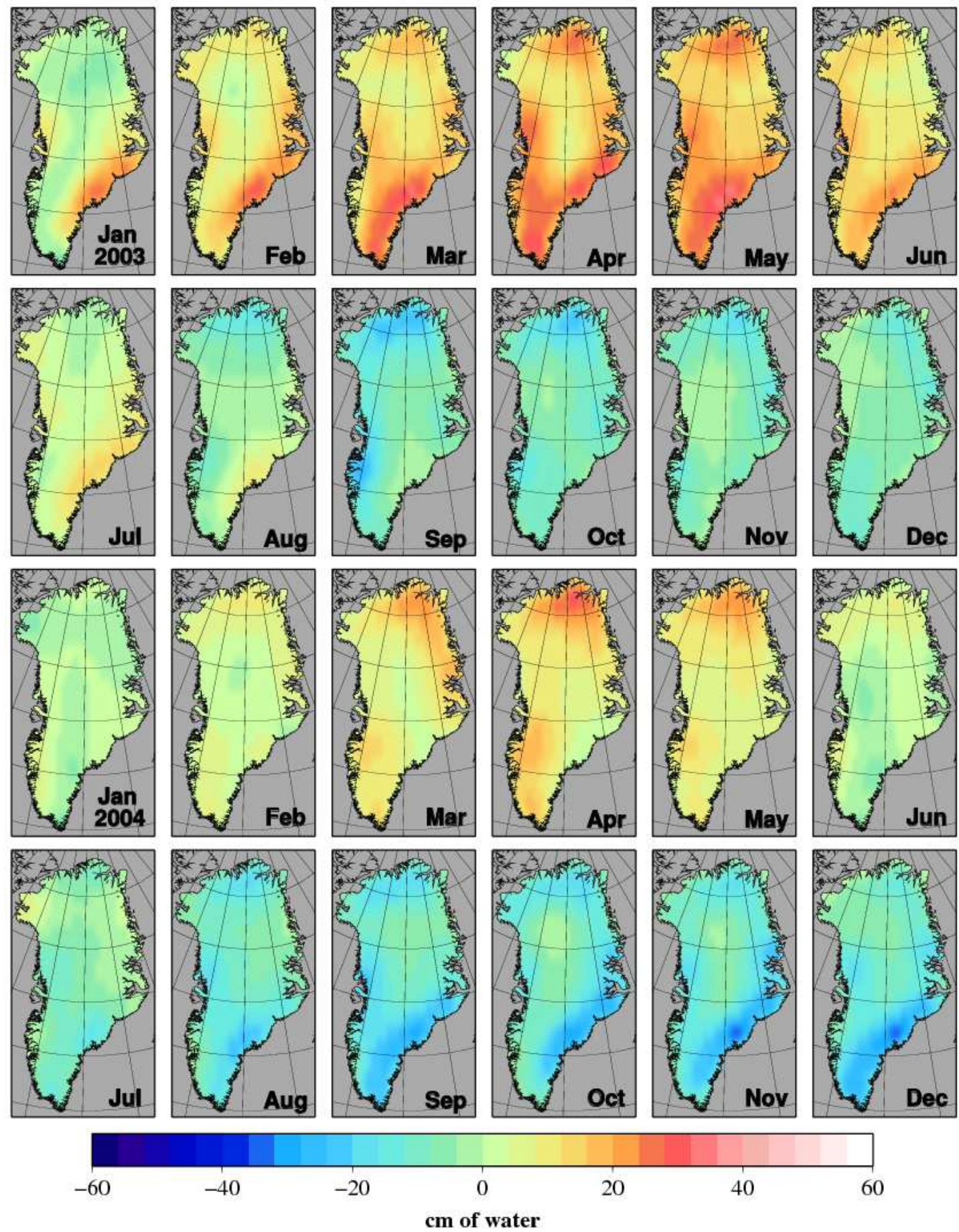

Figure 5.8: Greenland gravity estimates for separate land and ocean constraints. 


\subsubsection{Uncorrelated Basin Mascons}

The results presented here are for a set of uncorrelated basin mascon estimates. This is implemented by only constraining mascons to each other that are within the same basin. For this scenario the number of constraint matrices is equal to the number of basins over the land, plus one for the ocean. This is effectively the same as solving for a small number of uncorrelated mascons which are defined by the basins.

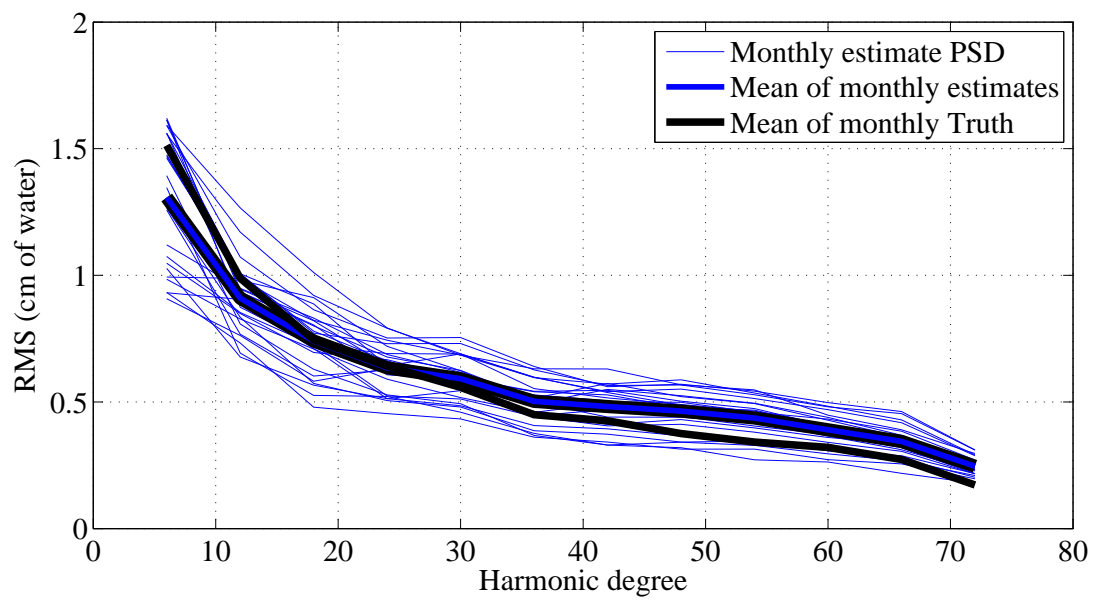

(a) South America

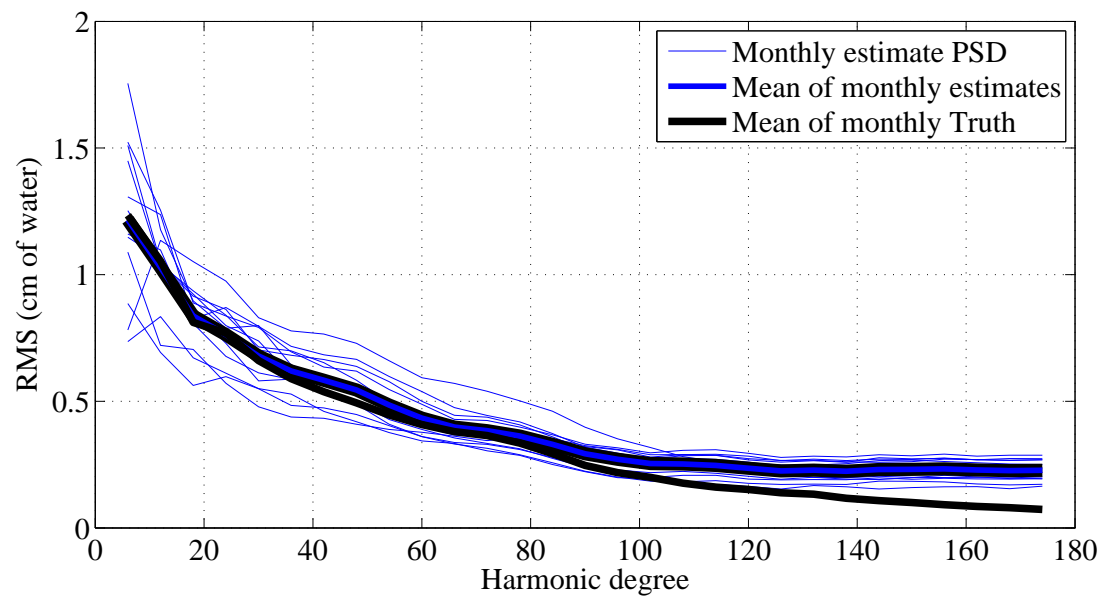

(b) Greenland

Figure 5.9: Spectral density of spatial maps in (a) Figure 5.10 and (b) Figure 5.11. 

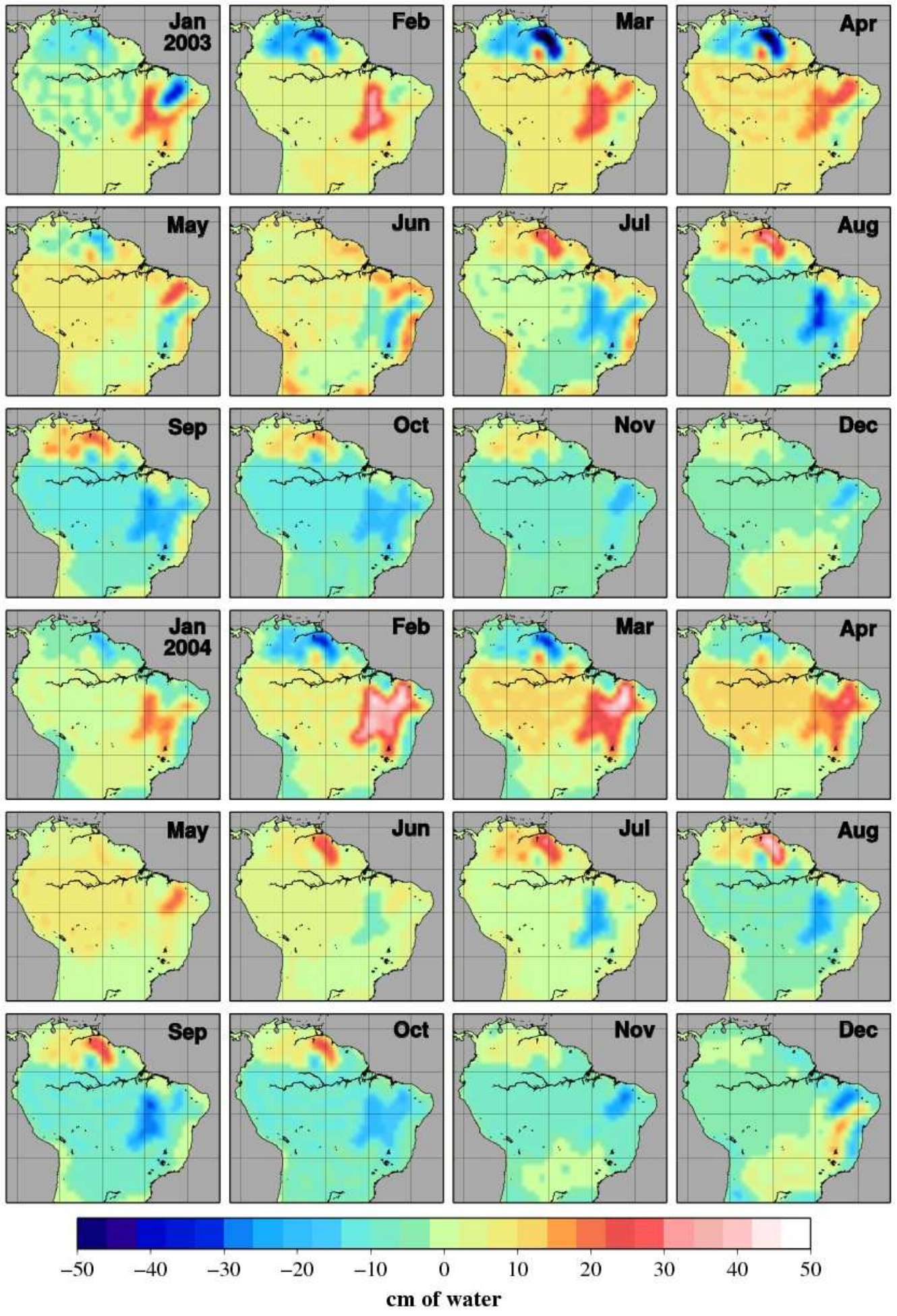

Figure 5.10: South America gravity estimates for uncorrelated basins. 

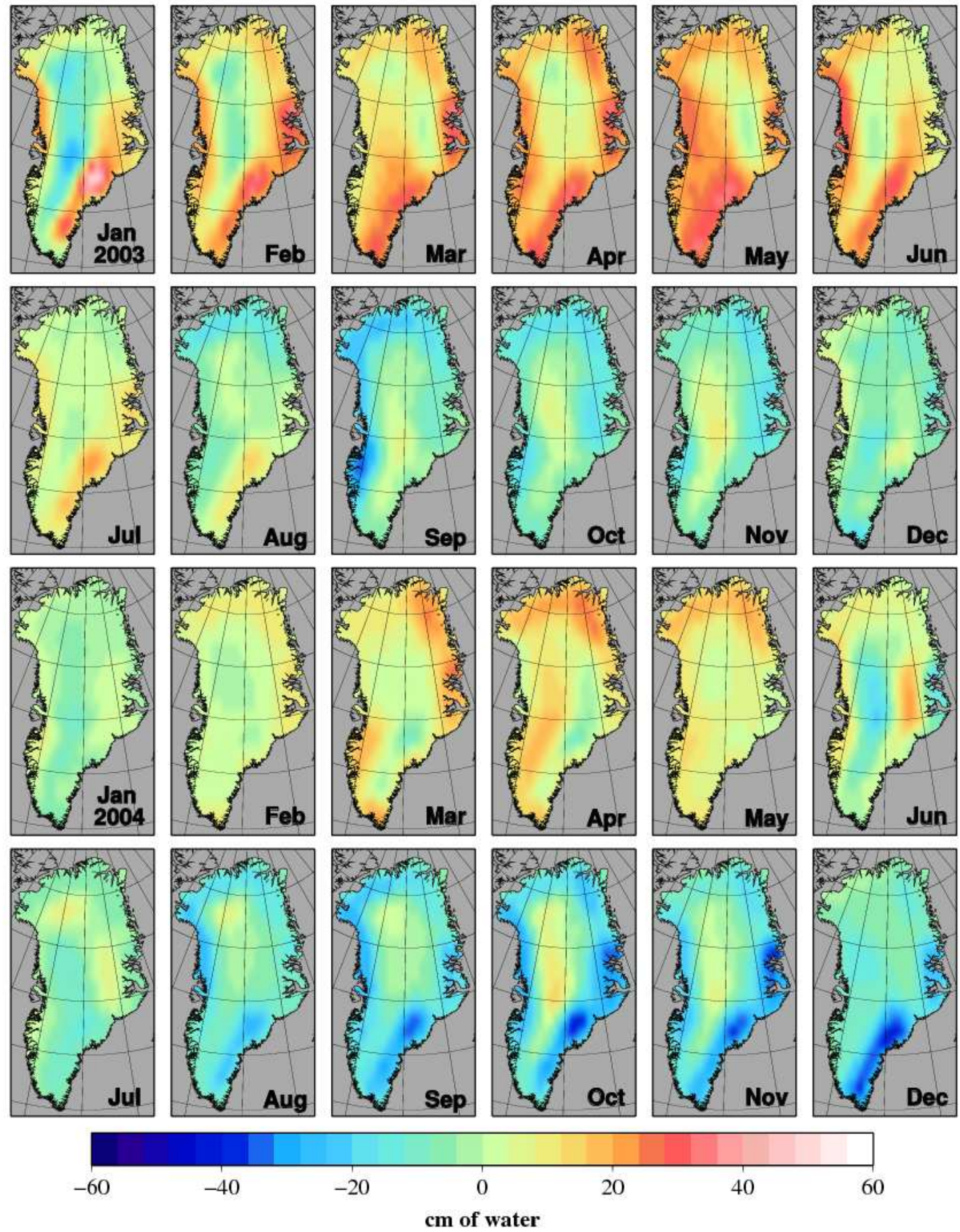

Figure 5.11: Greenland gravity estimates for uncorrelated basins. 


\subsection{4 $1^{\circ} \times 1^{\circ}$ Mascon Grid: Greenland Elevation Constraints}

The constraint scenario applied in this section only applies to the Greenland gravity estimates. As previously described, a benefit of the mascon approach is to set up the constraints such that mascons whose signals are uncorrelated are not constrained to one another. The Truth signal for mass change over Greenland has a sharp dividing line at $2000 \mathrm{~m}$ elevation defining the size of the amplitude of the annual signal. Mascons which neighbor each other across this line are constrained to each other for the results of Sections 5.3.1 and 5.3.2, even though they are known to have very different geophysical signals. This provides an opportunity to determine whether properly assigned additional constraints can improve the accuracy of the gravity solutions. The results in this section introduce an additional constraint matrix from Section 5.3.2, so that only mascons within the same elevation range (above or below $2000 \mathrm{~m}$ ) are constrained to each other.

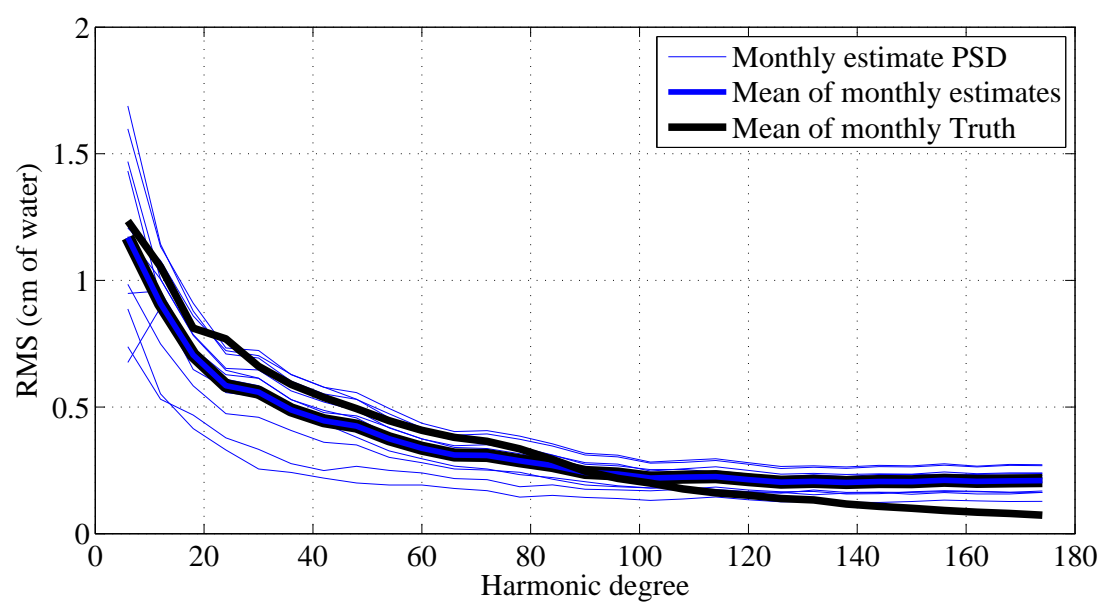

Figure 5.12: Spectral density of spatial maps in Figure 5.13. 

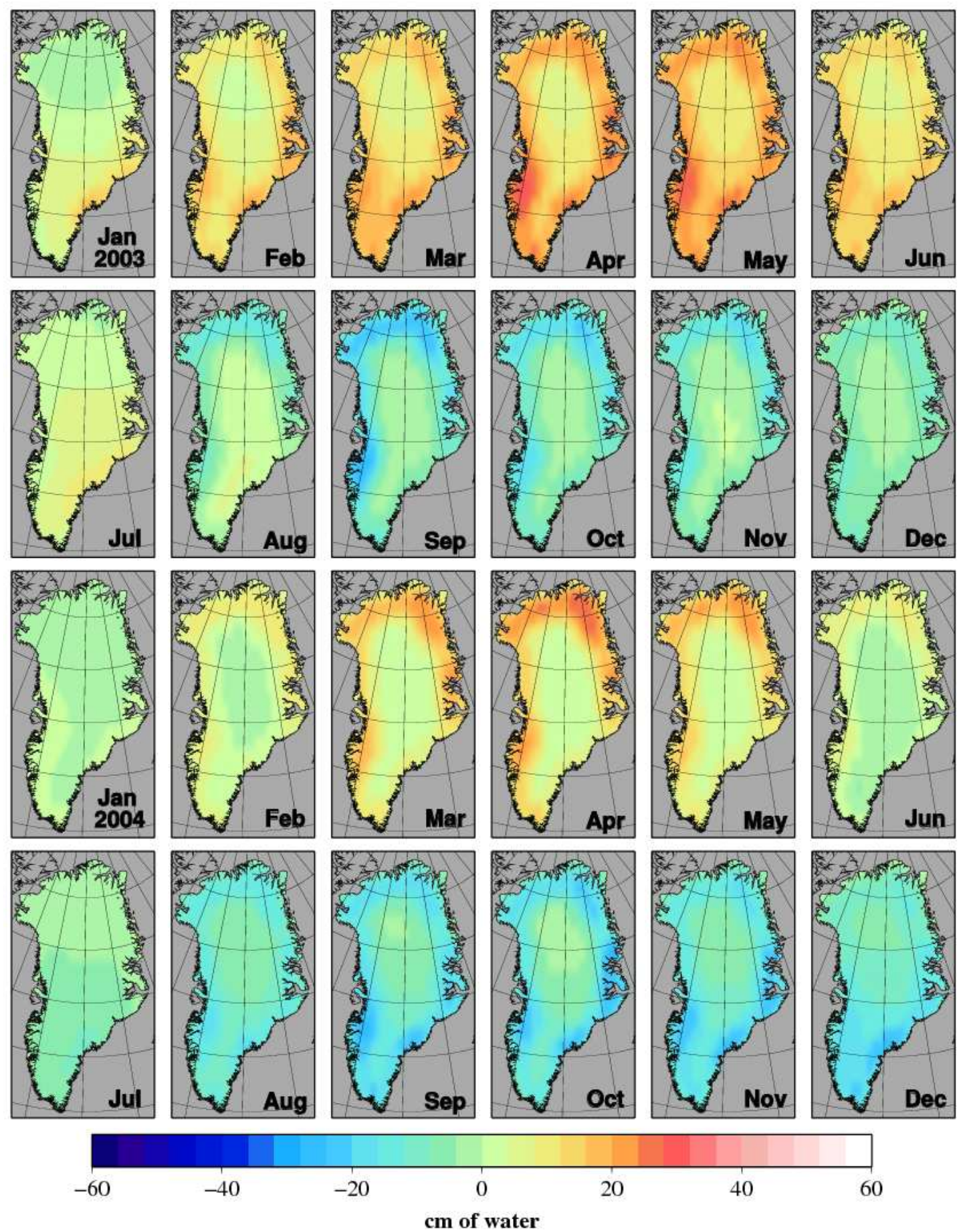

Figure 5.13: Greenland gravity estimates for elevation constraint scenario. 


\subsubsection{Comparison of Mascon Constraint Scenarios}

The results obtained from the different mascon constraint scenarios are compared by measuring the mass variations within each basin as calculated by each constraint scenario. The time series for South America and Greenland basin mass changes are presented in Figure 5.14 and Figure 5.15 respectively. Tables 5.1 and 5.2 show the corresponding error RMS values for the basin mass estimates.

The gravity estimate plots of Figures 5.4 and 5.5 and the mass change time series show that the one constraint only case results in a rather smoothed gravity estimate as compared to the other approaches. Since for this case there is no differentiation in how the mascons are treated, the signals over the land and ocean mascons leak into each other. This causes the one constraint matrix case to underestimate the signal for mascons located near the coast. This explains why the mass change estimates for the Amazon and Parana basins are good, as these basins are large in size and most of the signal is not located near the coast. This constraint case also clearly underestimates the amplitude of the signal for the coastal Greenland basins (1-6) as compared to the other cases.

The case where the land and ocean mascons are constrained separately provides clear improvement in the mass change estimates for basins located near the coast for both South America and Greenland as compared to the one constraint matrix case. For South America, this case provides the lowest error RMS for most of the basins, and the RMS values are not significantly higher for cases where it is not the lowest. This mascon constraint setup is clearly the best option for forming the South America mascon solutions. 

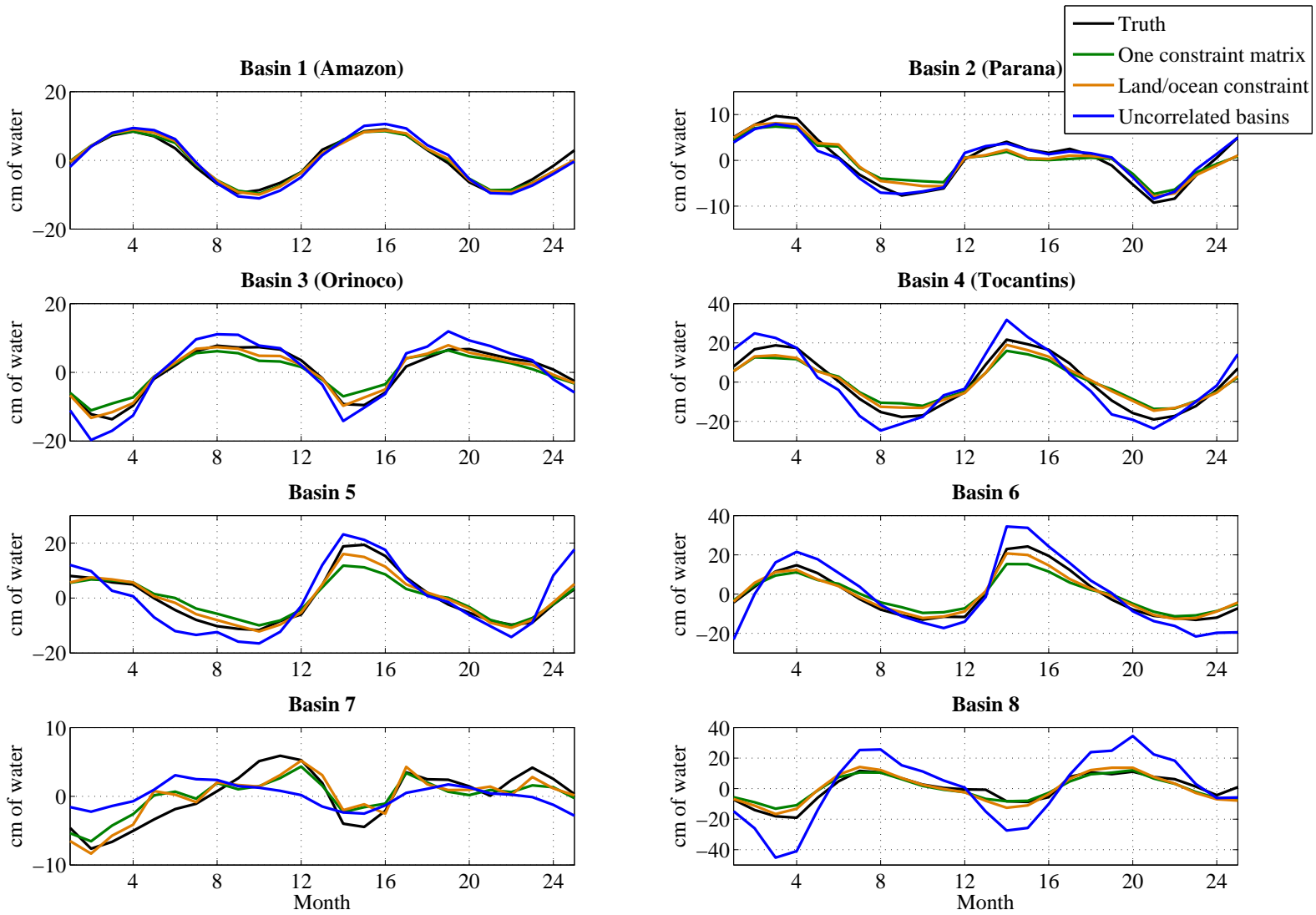

Figure 5.14: South America basin mass variations time series for different mascon constraint scenarios.

\begin{tabular}{|l||l||l|l|l|}
\hline Basin & $\begin{array}{l}\text { Truth } \\
\text { signal RMS } \\
\text { (cm of water) }\end{array}$ & $\begin{array}{l}\text { One constraint } \\
\text { matrix } \\
\text { error RMS } \\
\text { (cm of water) }\end{array}$ & $\begin{array}{l}\text { Land/ocean } \\
\text { constraint } \\
\text { error RMS } \\
\text { (cm of water) }\end{array}$ & $\begin{array}{l}\text { Uncorrelated } \\
\text { basins } \\
\text { error RMS } \\
\text { (cm of water })\end{array}$ \\
\hline 1 & 6.222 & 0.979 & 1.048 & 1.674 \\
\hline 2 & 5.459 & 1.960 & 1.623 & 1.123 \\
\hline 3 & 6.705 & 2.193 & 1.268 & 3.181 \\
\hline 4 & 13.609 & 4.515 & 3.583 & 5.483 \\
\hline 5 & 9.076 & 3.333 & 1.883 & 5.241 \\
\hline 6 & 11.844 & 3.985 & 2.309 & 6.930 \\
\hline 7 & 3.790 & 1.905 & 1.810 & 3.395 \\
\hline 8 & 8.907 & 3.484 & 3.579 & 13.166 \\
\hline
\end{tabular}

Table 5.1: RMS of errors for South America basin mass estimates for different mascon constraint scenarios shown in Figure 5.14. 

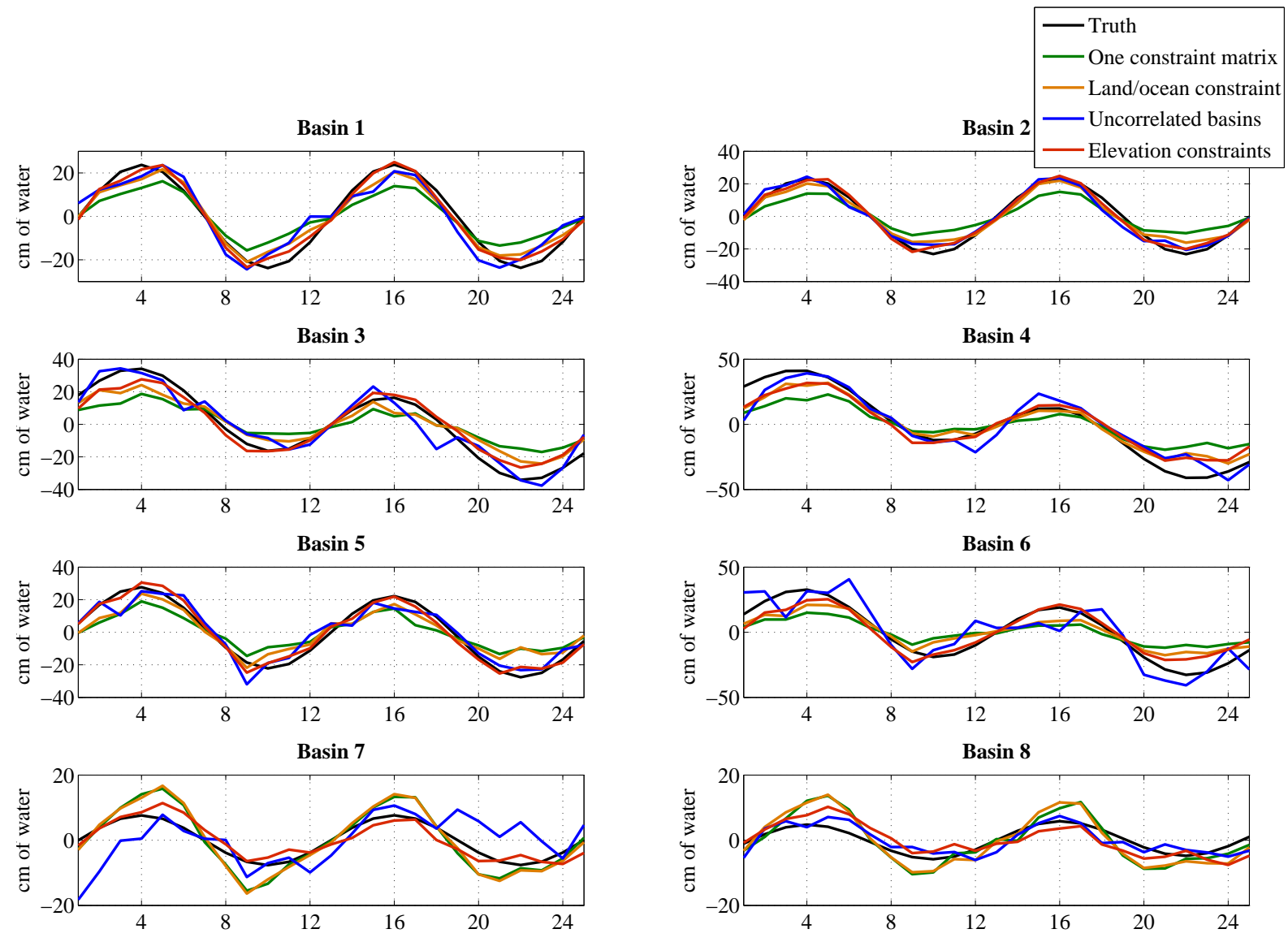

Basin 9
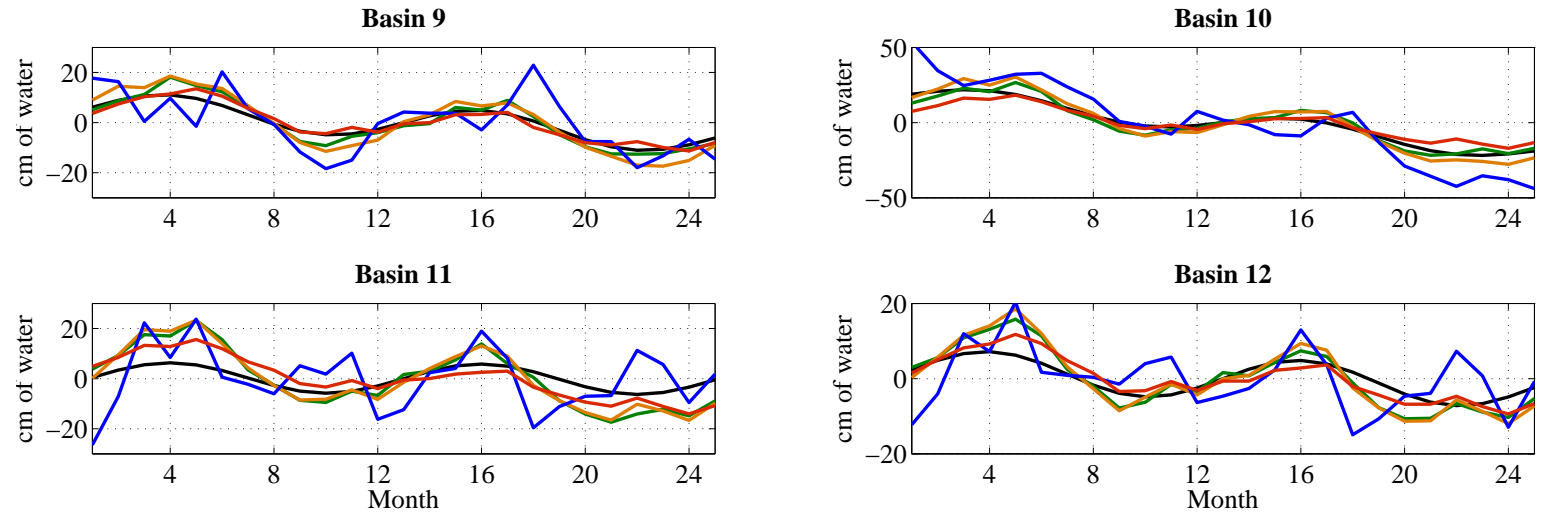

Figure 5.15: Greenland basin mass variations time series for different mascon constraint scenarios. 


\begin{tabular}{|l||l||l|l|l|l|}
\hline Basin & $\begin{array}{l}\text { Truth } \\
\text { signal RMS } \\
\text { (cm of water) }\end{array}$ & $\begin{array}{l}\text { One constr. } \\
\text { matrix } \\
\text { error RMS } \\
\text { (cm of water) }\end{array}$ & $\begin{array}{l}\text { Land/ocean } \\
\text { constraint } \\
\text { error RMS } \\
\text { (cm of water) }\end{array}$ & $\begin{array}{l}\text { Uncorrelated } \\
\text { basins } \\
\text { error RMS } \\
\text { (cm of water) }\end{array}$ & $\begin{array}{l}\text { Elevation } \\
\text { constraints } \\
\text { error RMS } \\
\text { (cm of water) }\end{array}$ \\
\hline 1 & 16.513 & 7.553 & 4.356 & 5.746 & 2.699 \\
\hline 2 & 16.306 & 7.686 & 3.905 & 3.365 & 2.337 \\
\hline 3 & 20.708 & 11.231 & 7.731 & 6.795 & 5.544 \\
\hline 4 & 24.937 & 13.483 & 8.157 & 8.891 & 8.131 \\
\hline 5 & 17.647 & 9.074 & 7.262 & 5.876 & 3.196 \\
\hline 6 & 20.325 & 12.505 & 9.235 & 11.321 & 6.838 \\
\hline 7 & 5.145 & 4.489 & 4.667 & 6.938 & 2.605 \\
\hline 8 & 3.628 & 4.179 & 4.376 & 2.538 & 3.387 \\
\hline 9 & 7.241 & 3.057 & 4.480 & 8.459 & 2.028 \\
\hline 10 & 14.295 & 3.969 & 5.190 & 13.972 & 4.758 \\
\hline 11 & 4.258 & 7.861 & 8.102 & 11.823 & 5.910 \\
\hline 12 & 4.496 & 3.994 & 4.832 & 7.888 & 2.833 \\
\hline
\end{tabular}

Table 5.2: RMS of errors for Greenland basin mass estimates for different mascon constraint scenarios shown in Figure 5.15.

The uncorrelated basins setup results in estimates with much larger mass variations than the previous two cases. The mass change time series and error RMS values reveal that this is clearly not a good option for forming the solutions. Simulations (results not presented) showed that these large errors are primarily the result of a magnification of the temporal aliasing error.

The elevation constraint setup for Greenland results in the most accurate gravity estimates. The error RMS is lowest for this case for eleven out of the twelve basins, and the mass change time series show that the elevation case more accurately determines the amplitude of the signal for almost every basin. The results of this scenario confirm that the mascon approach can be setup to account for the fact that certain mascons should or should not be constrained to each other based on the geophysical signals occurring within their boundaries. The Truth signal for Greenland in these simulations has large difference for neighboring mascons which are divided by the $2000 \mathrm{~m}$ elevation line. 
For the one constraint matrix and the separate land and ocean constraint cases, these neighboring mascons are constrained to each other. This causes the neighboring signals to leak, which is evident in the fact that the gravity signals within the coastal basins (16) are underestimated while the signals for the interior basins (7-12) are overestimated for these cases. The elevation constraint approach eliminates this problem which results in much improved gravity estimates.

The results presented in the following two sections utilize the separate land and ocean constraints case for forming the South America gravity solutions and the elevation constraints for forming Greenland solutions.

\subsection{Simulated Gravity Estimates: Instrument Noise Only}

The results presented in this section are for the instrument noise only case. These simulations account for the satellite-to-satellite ranging instrument errors, the satellite positioning errors, and the accelerometer or GRS errors only. The results given here ignore the temporal aliasing errors caused by mis-modeling of the atmospheric, oceanographic, and tidal models so the accuracy of the gravity estimates will be optimistic. The results here are instructional, however, as they provide valuable information of the effects of the different instruments on board the various simulated missions. Temporal aliasing errors will still be present in the South America gravity estimates due to the high frequency variations in the hydrology signal that is estimated at monthly intervals. Aliasing from the atmosphere, oceans, and tides are added to the simulations in Section 5.5 . 


\subsubsection{South America}

Spatial maps of the gravity estimates for the noise only case over South America are given on the following pages. The results for GRACE, GFO Case 1, GFO Case 2, and GFO Case 3 are shown in Figures 5.16, 5.17, 5.18, and 5.19 respectively. The gravity estimates for GRACE and GFO Case 1 appear to be nearly identical, as do the estimates for GFO Case 2 and GFO Case 3. 

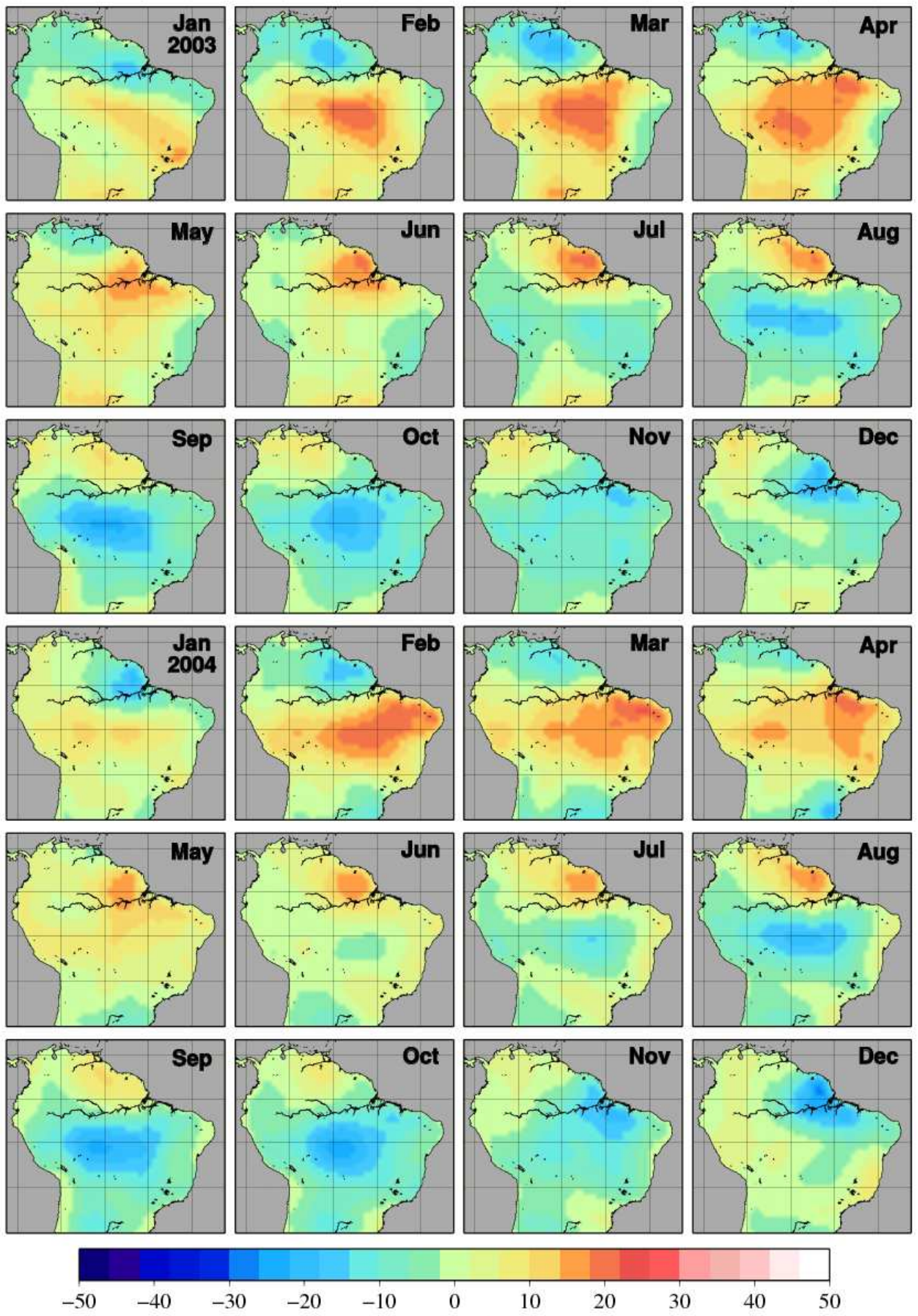

cm of water

Figure 5.16: South America gravity estimates for GRACE (480 km altitude / K-band / Accelerometer): instrument noise only. 

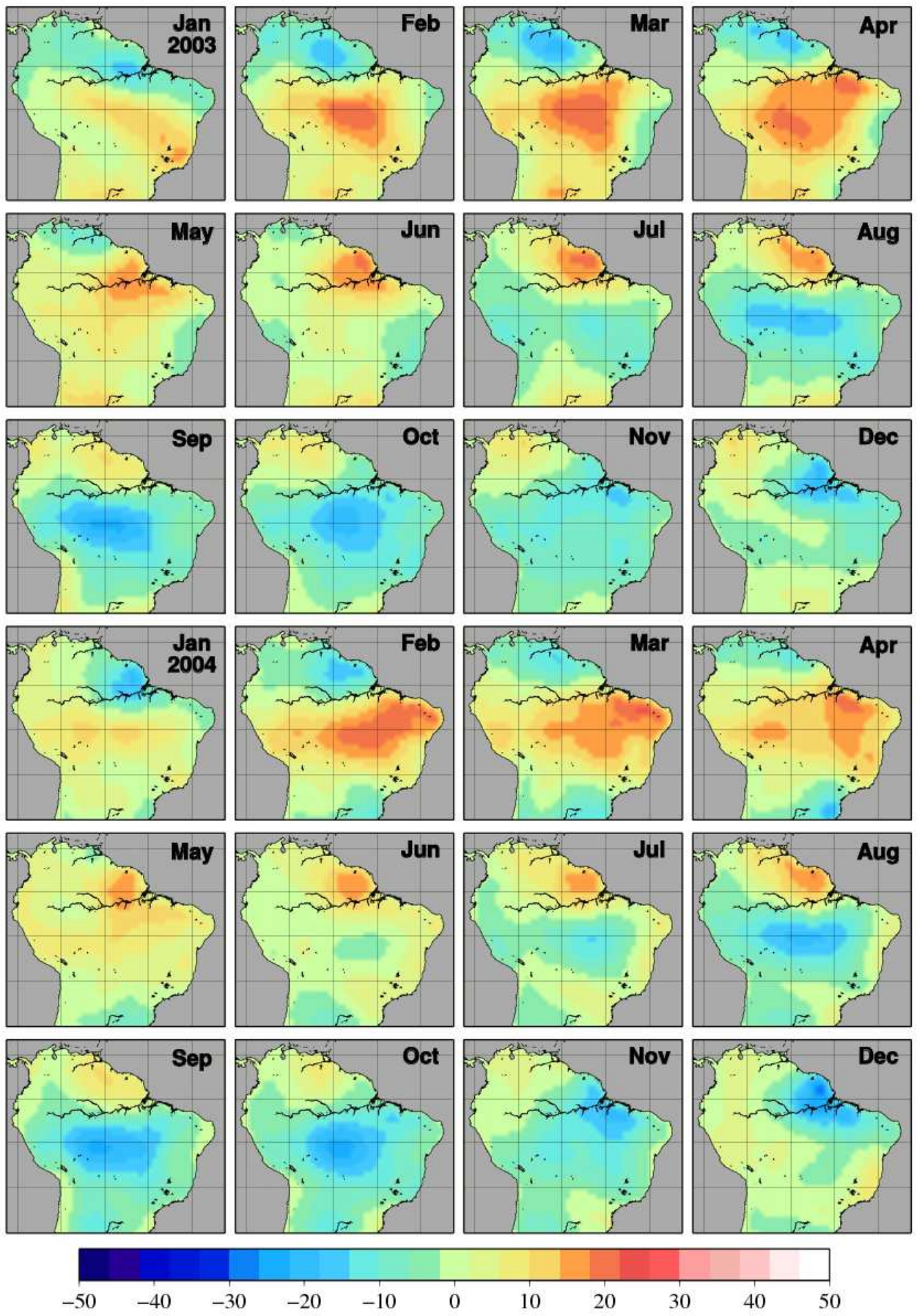

cm of water

Figure 5.17: South America gravity estimates for GFO Case 1 (480 km altitude / Laser / Accelerometer): instrument noise only. 

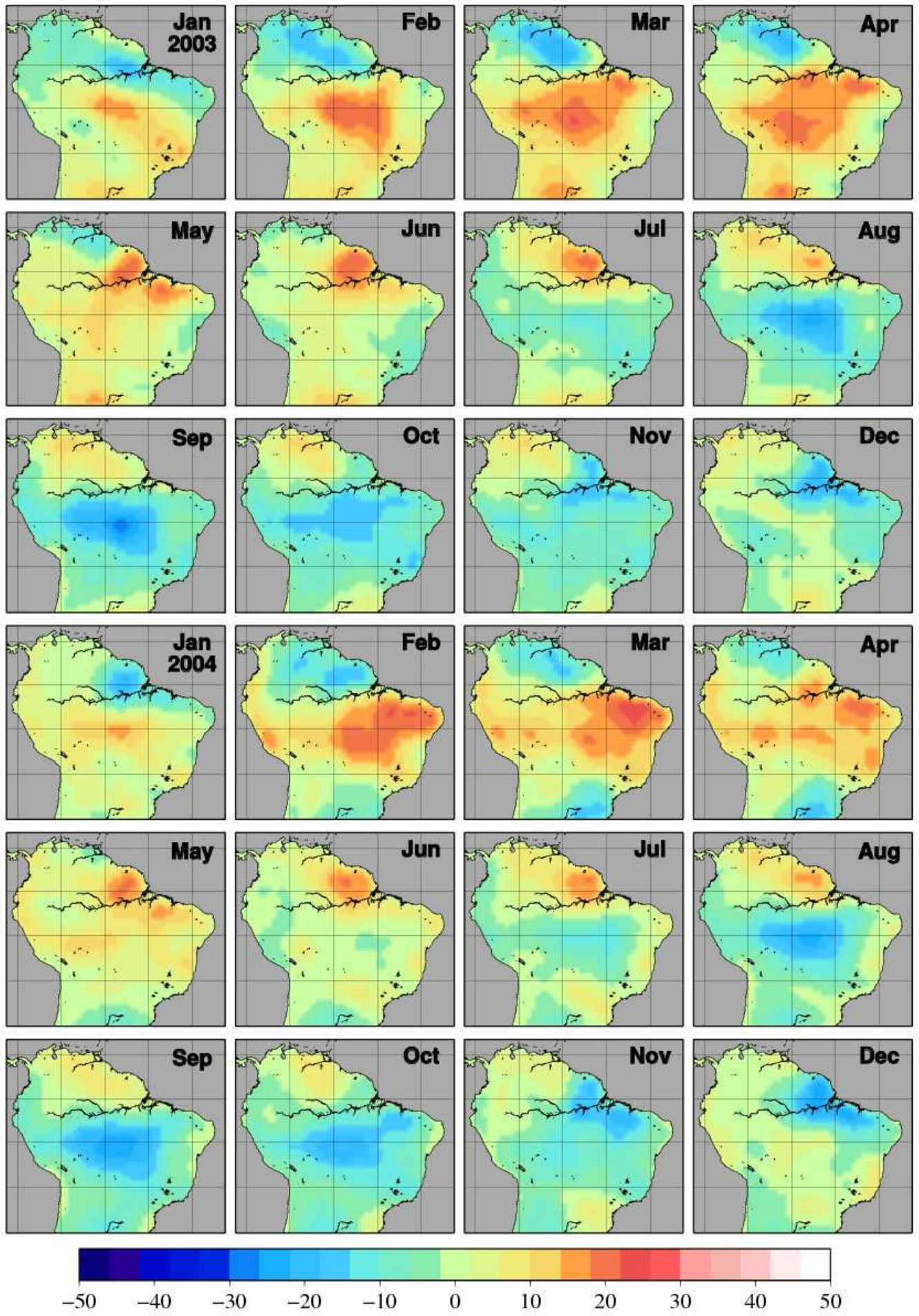

cm of water

Figure 5.18: South America gravity estimates for GFO Case 2 (250 km altitude / Kband / GRS): instrument noise only. 

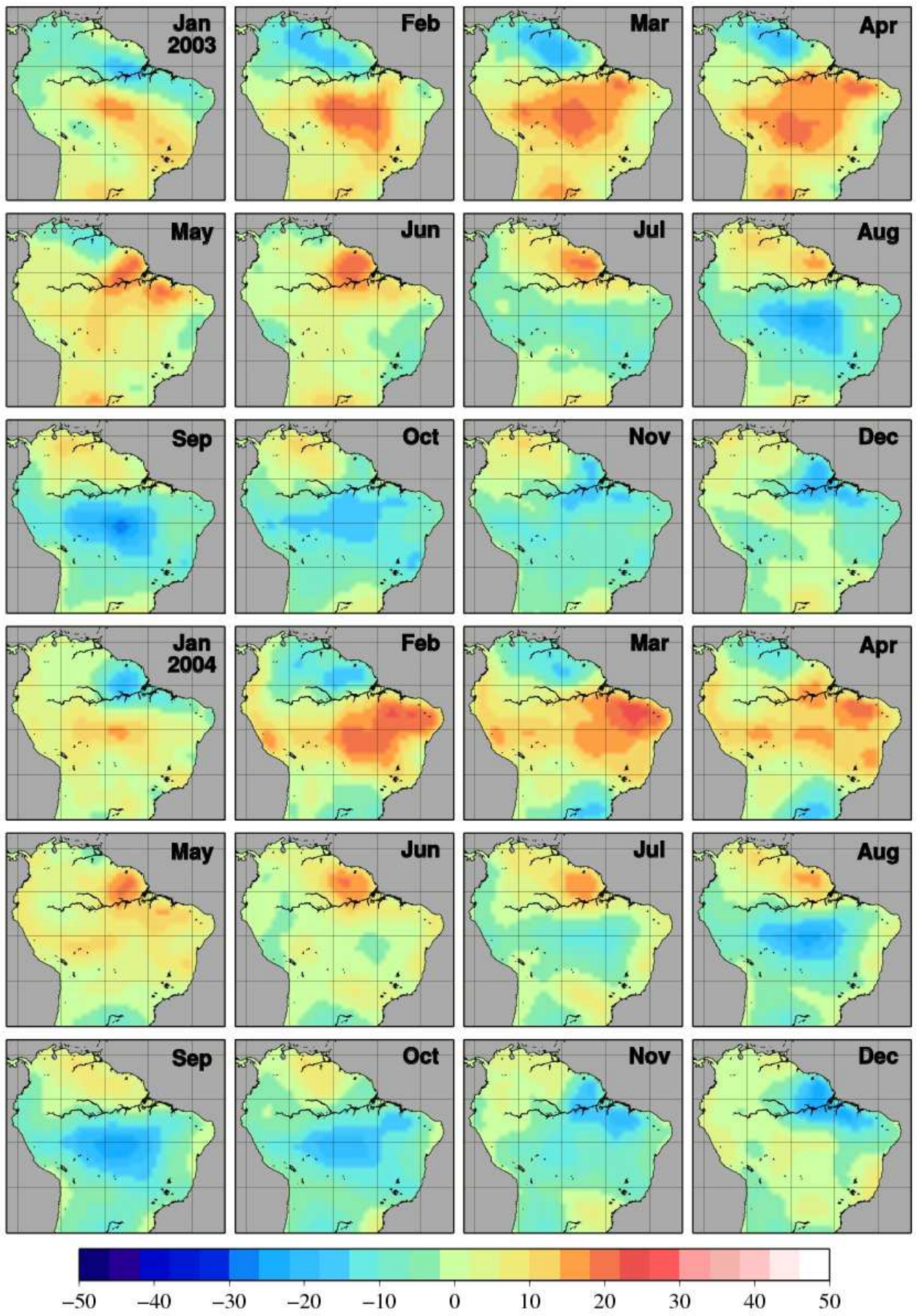

cm of water

Figure 5.19: South America gravity estimates for GFO Case 3 (250 km altitude / Laser / GRS): instrument noise only. 


\subsubsection{Greenland}

The Greenland gravity estimate maps for the noise only case are plotted on the following pages. Results for GRACE, GFO Case 1, GFO Case 2, and GFO Case 3 are shown in Figures 5.20, 5.21, 5.22, and 5.23 respectively. As was the case for the South America simulations, the gravity estimates for GRACE and GFO Case 1 look identical, while the GFO Case 2 and GFO Case 3 results show only slight differences. 

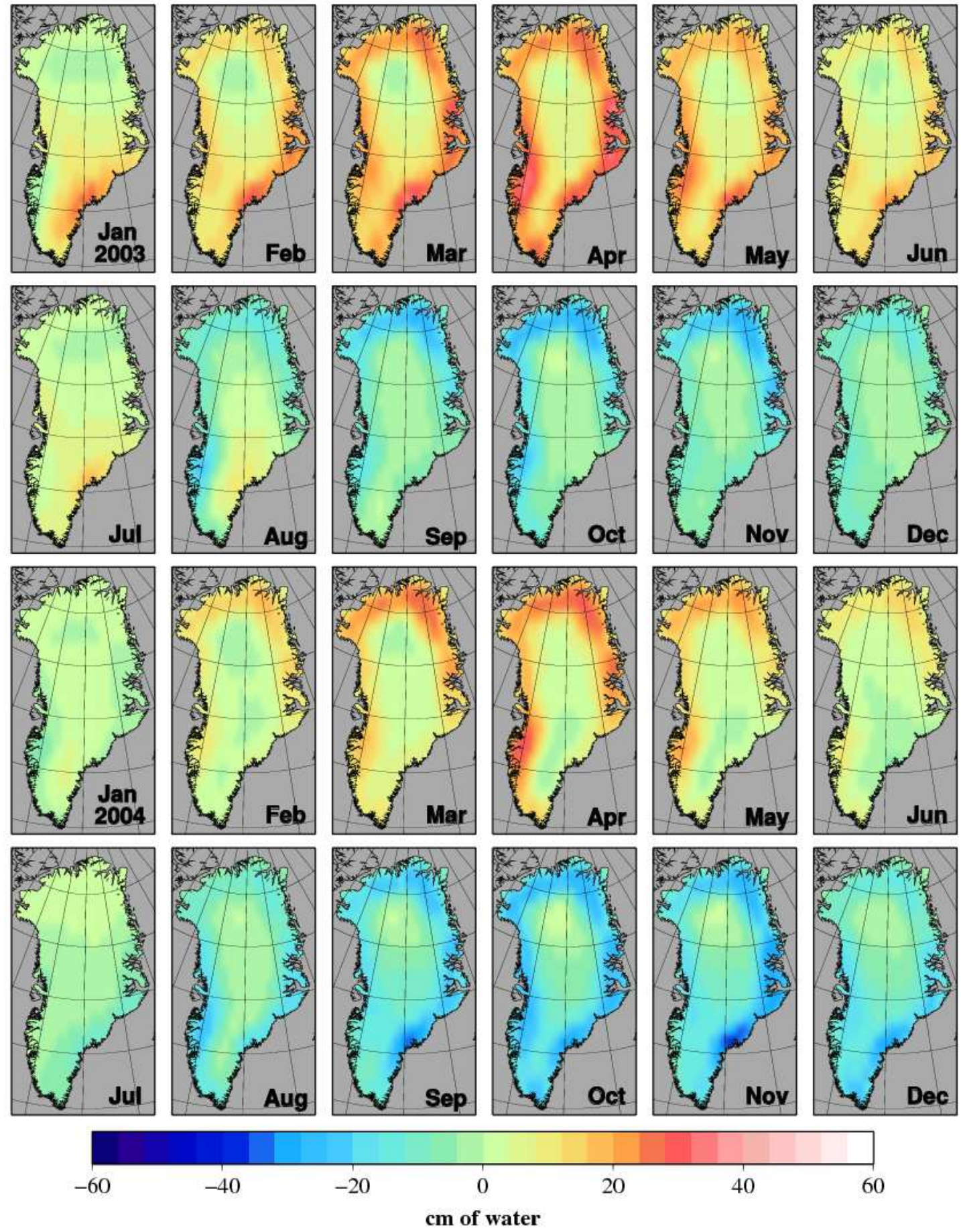

Figure 5.20: Greenland gravity estimates GRACE (480 km altitude / K-band / Accelerometer): instrument noise only. 

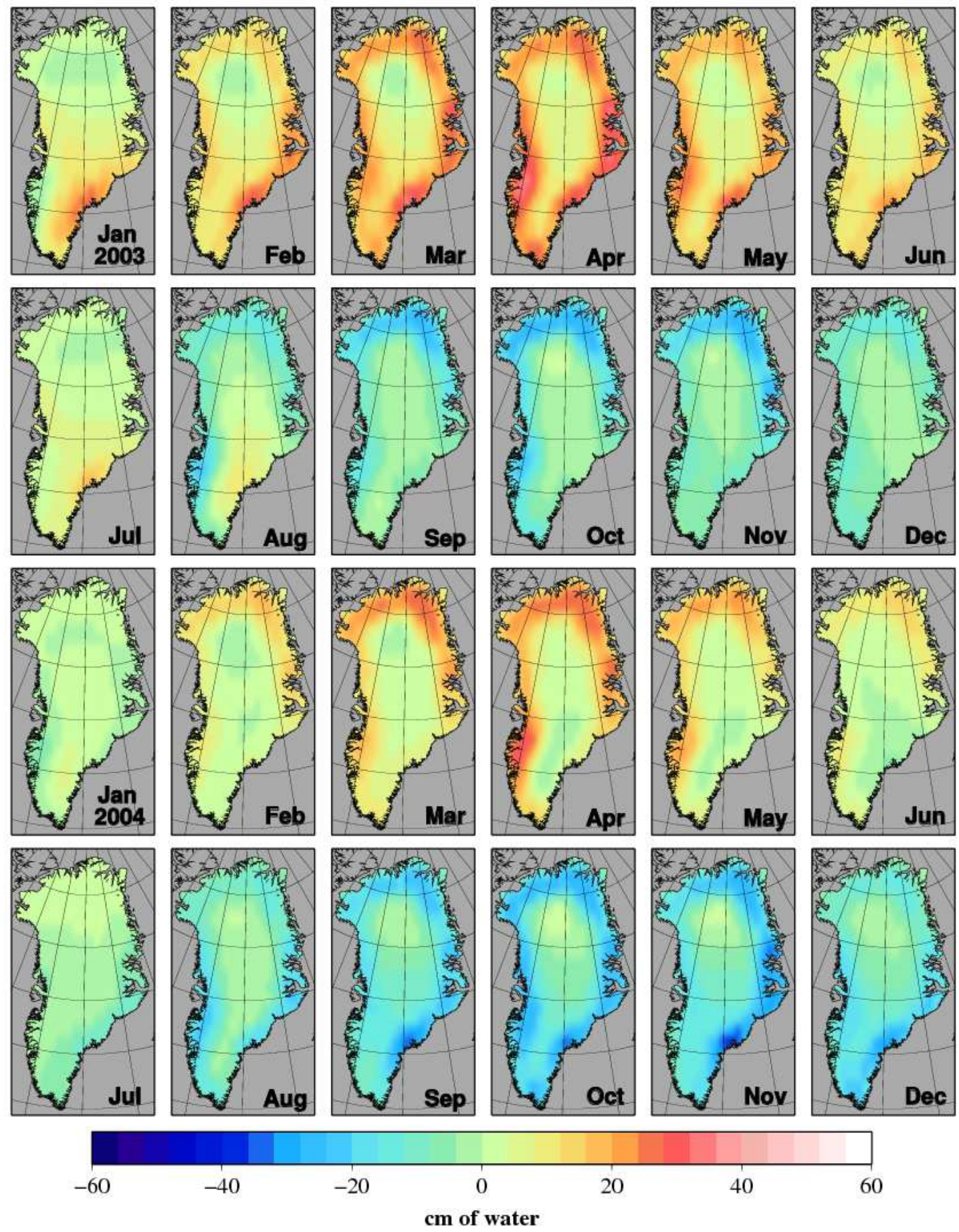

Figure 5.21: Greenland gravity estimates GFO Case 1 (480 km altitude / Laser / Accelerometer): instrument noise only. 

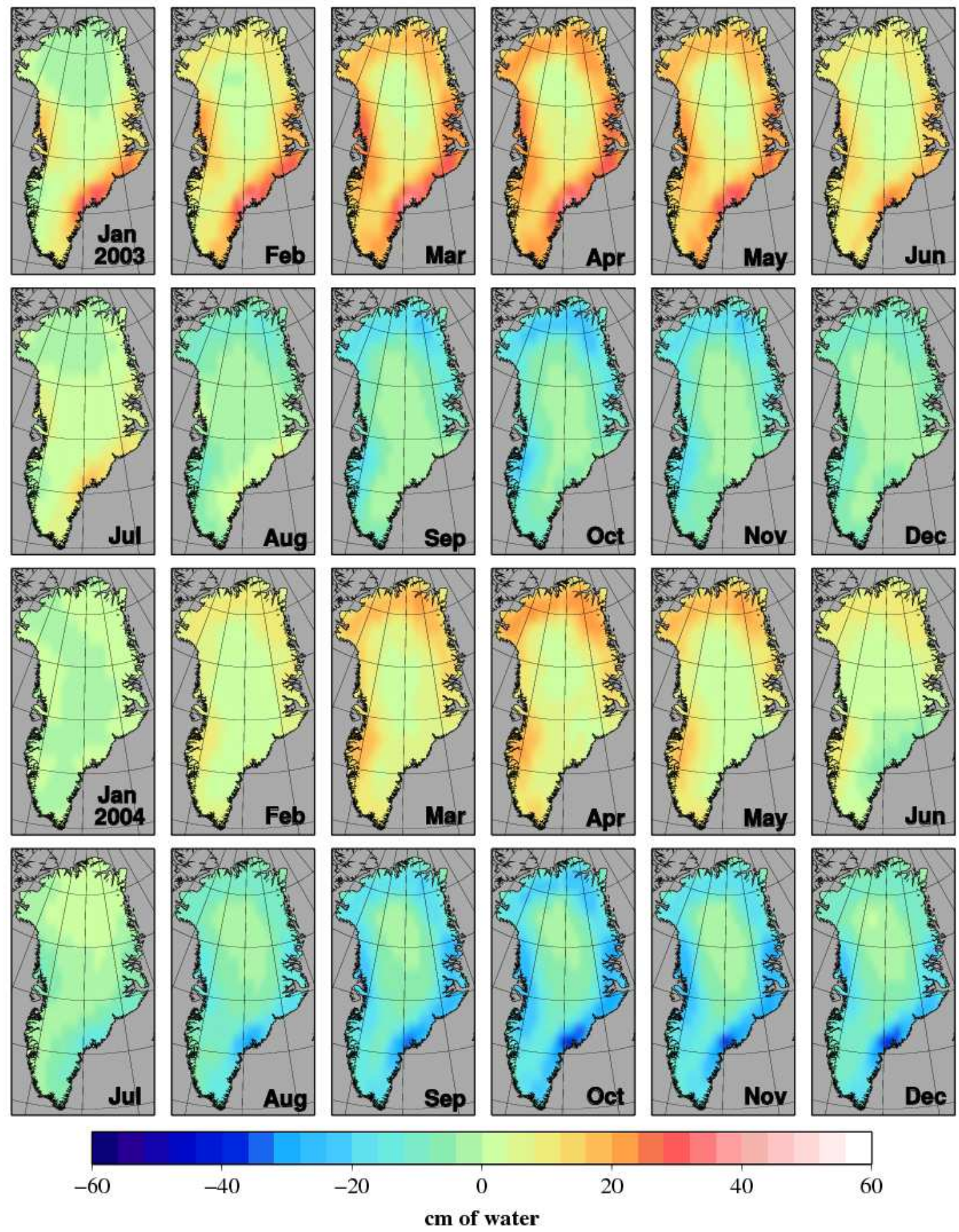

Figure 5.22: Greenland gravity estimates for GFO Case 2 (250 km altitude / K-band / GRS): instrument noise only. 

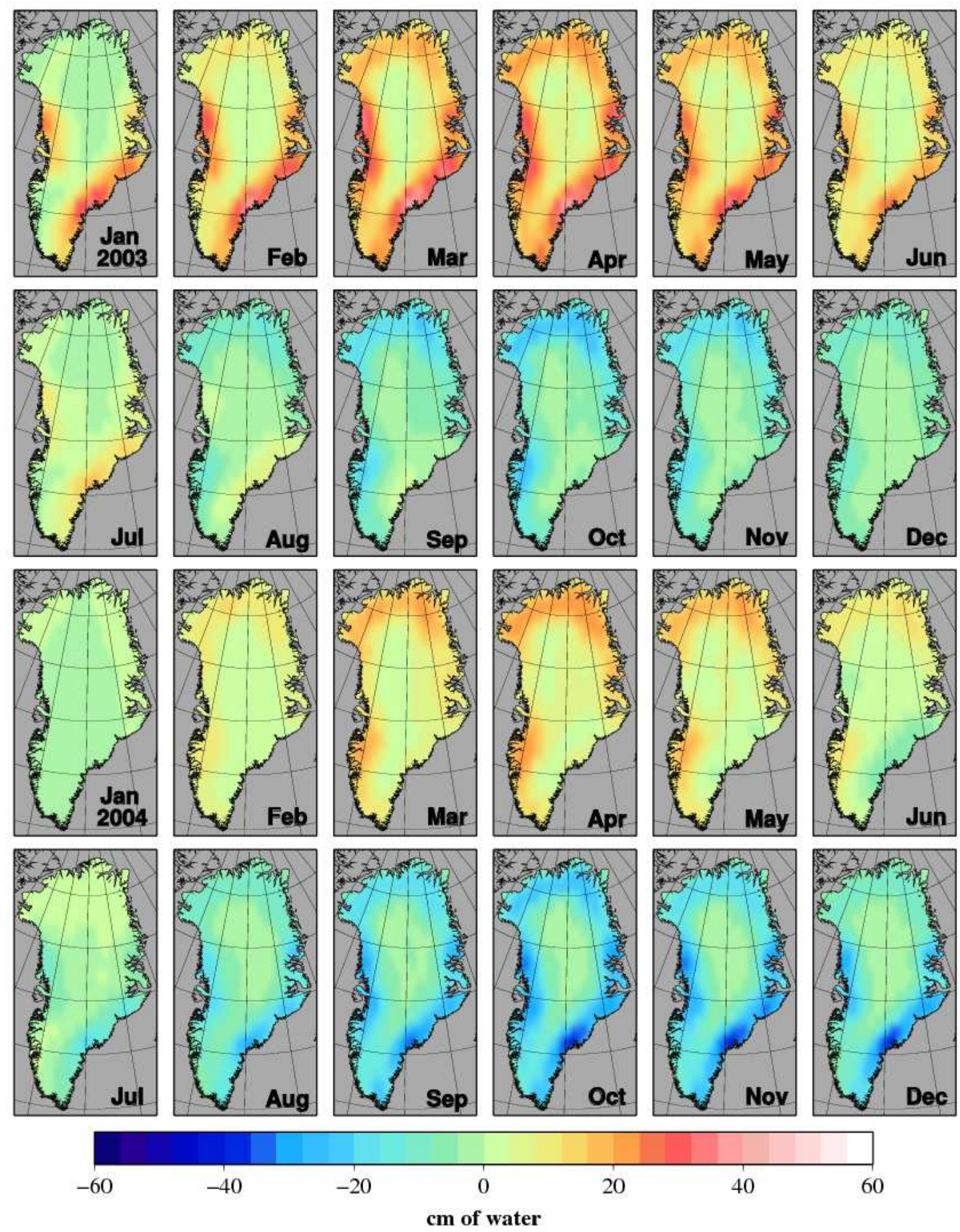

Figure 5.23: Greenland gravity estimates for GFO Case 3 (250 km altitude / Laser / GRS): instrument noise only. 


\subsubsection{Comparison of Mission Configurations}

\subsubsection{South America}

The spatial RMS of the errors for each monthly gravity estimate for the four simulated missions is plotted in Figure 5.24. The mean of the 25 monthly RMS values for each mission is given in Table 5.3. These results show that there is essentially no difference between the GRACE and GFO Case 1 mission performance, and the GFO Case 2 and GFO Case 3 mission performance for recovering the time-variable GLDAS hydrology signal over South America for the instrument noise only case. Due to similarities in performance to the other missions, only the results for GRACE and GFO Case 3 are considered for the remainder of this subsection.

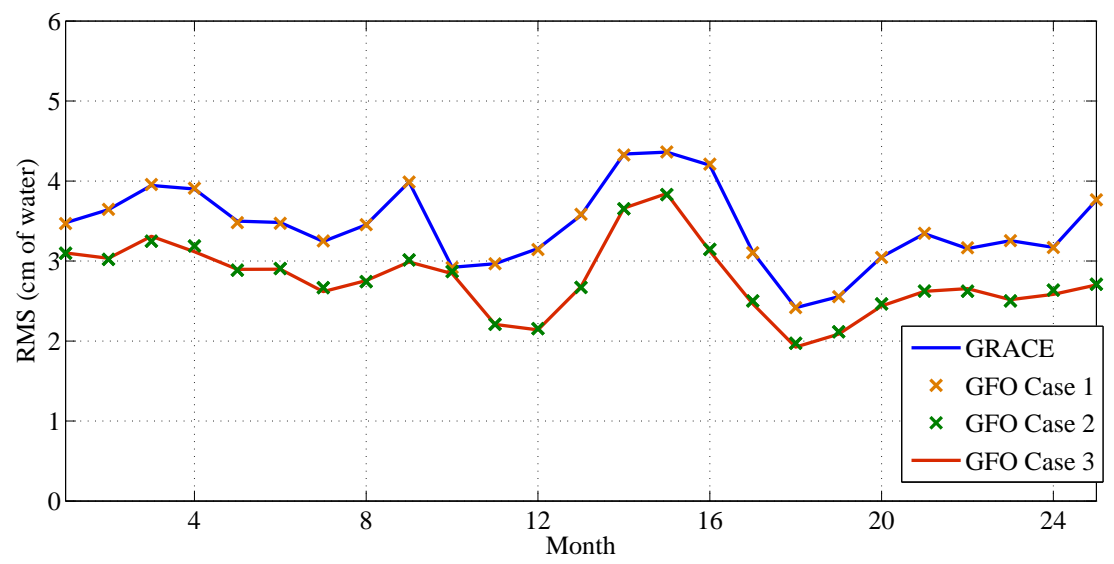

Figure 5.24: RMS errors of South America spatial maps for all mission conifigurations: instrument noise only case. 


\begin{tabular}{|l|l|}
\hline $\begin{array}{l}\text { Mission } \\
\text { Configuration }\end{array}$ & $\begin{array}{l}\overline{\mathbf{R M S}} \\
\text { (cm of water) }\end{array}$ \\
\hline GRACE & 3.44 \\
\hline GFO Case 1 & 3.44 \\
\hline GFO Case 2 & 2.78 \\
\hline GFO Case 3 & 2.77 \\
\hline
\end{tabular}

Table 5.3: Mean of monthly RMS of 25 South America spatial plots for instrument noise only case.

The mean spectral density of the monthly spatial South America maps for the Truth signal and the GRACE and GFO Case 3 errors are plotted in Figure 5.25. There is a noticeable improvement for GFO Case 3 at the spatial frequencies described by harmonic degrees 18 to 42 , while little to no improvement is seen for the lowest and highest sampled frequencies. At the highest frequencies the power of the errors is approximately equal to the signal size.

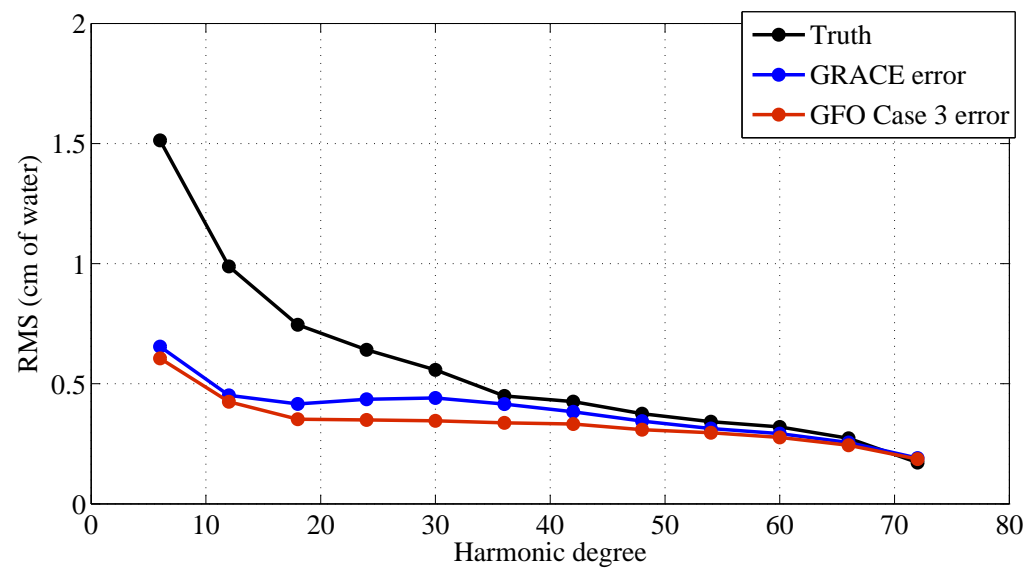

Figure 5.25: Mean spectral density of monthly spatial maps for the Truth signal and GRACE and GFO Case 3 error over South America: instrument noise only.

The monthly gravity estimates for GRACE and GFO Case 3 are used to form time series of mass variations within each of the South American basins and is shown in Figure 5.26. The error RMS of each time series is given in Table 5.4. The gravity 
estimates for GFO Case 3 have a lower error RMS for seven of the eight basins.

The time series of mass variations are used to estimate a constant, trend, amplitude of annual signal, and phase of annual signal for the Truth data set, and the GRACE and GFO Case 3 estimates. These values are given in Table 5.5. The differences between the Truth and estimated values for trend and amplitude are plotted in Figure 5.27. The results show that neither simulated mission is consistently better than the other at recovering these components of the gravity signal. 

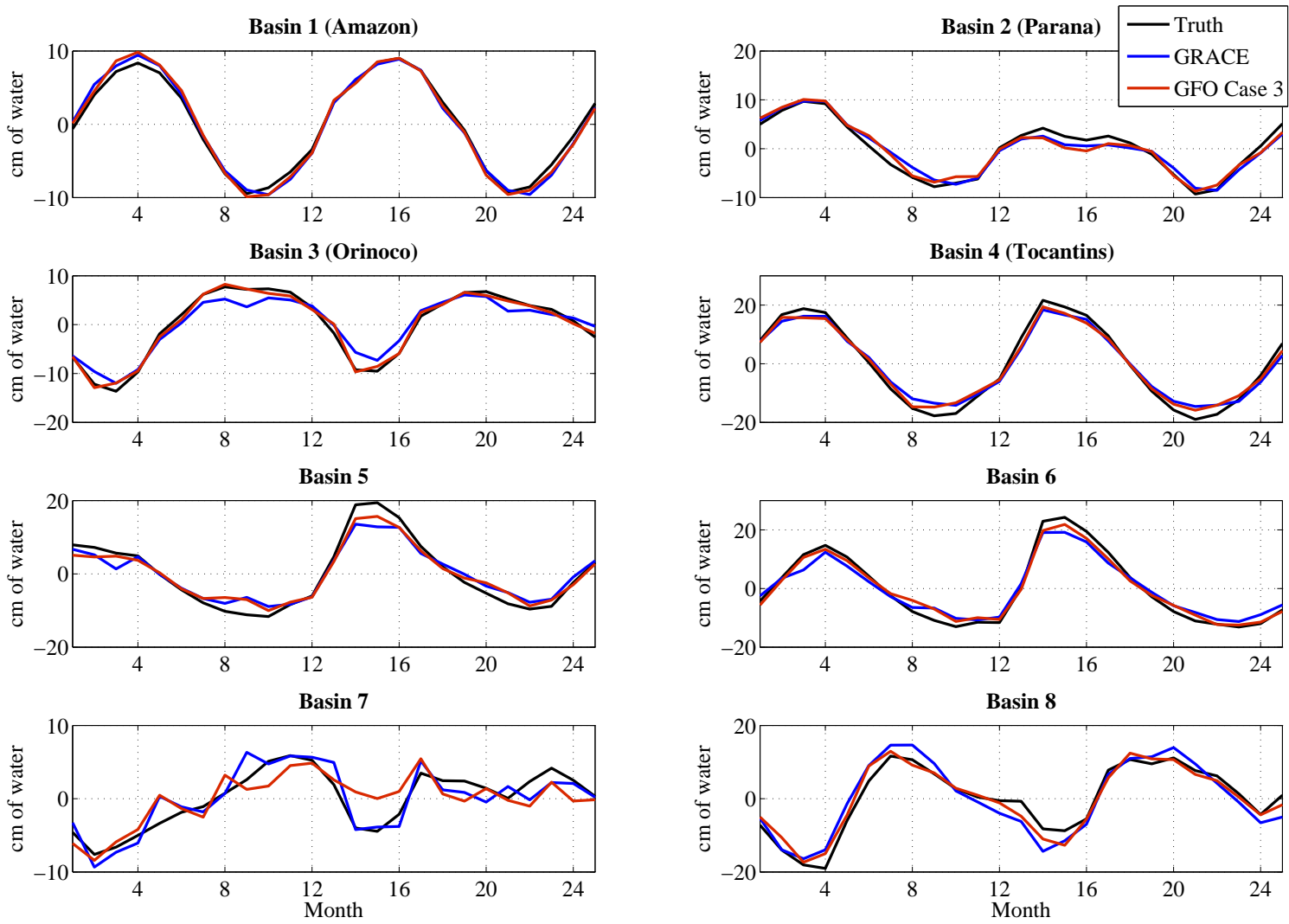

Figure 5.26: South America basin mass variations time series for different mission configurations: instrument errors only.

\begin{tabular}{|l||l||l|l|}
\hline Basin & $\begin{array}{l}\text { Truth } \\
\text { signal RMS } \\
\text { (cm of water) }\end{array}$ & $\begin{array}{l}\text { GRACE } \\
\text { error RMS } \\
\text { (cm of water) }\end{array}$ & $\begin{array}{l}\text { GFO Case 3 } \\
\text { error RMS } \\
\text { (cm of water) }\end{array}$ \\
\hline 1 & 6.222 & 0.748 & 0.728 \\
\hline 2 & 5.459 & 1.254 & 1.229 \\
\hline 3 & 6.705 & 1.840 & 0.752 \\
\hline 4 & 13.609 & 2.552 & 2.049 \\
\hline 5 & 9.076 & 2.625 & 2.138 \\
\hline 6 & 11.844 & 2.701 & 1.810 \\
\hline 7 & 3.790 & 1.682 & 2.322 \\
\hline 8 & 8.907 & 3.242 & 2.164 \\
\hline
\end{tabular}

Table 5.4: RMS of errors for South America basin mass estimates for different mission configurations shown in Figure 5.26: instrument errors only. 


\begin{tabular}{|c|c|c|c|c|c|c|c|c|c|}
\hline \multirow[t]{2}{*}{ Basin } & \multicolumn{3}{|c|}{ Truth } & \multicolumn{3}{|c|}{ GRACE } & \multicolumn{3}{|c|}{ GFO Case 3} \\
\hline & $\begin{array}{l}\text { Trend } \\
(\mathrm{cm})\end{array}$ & $\begin{array}{l}\text { Amp } \\
(\mathrm{cm} / \mathrm{yr})\end{array}$ & $\begin{array}{l}\text { Phase } \\
\text { (deg) }\end{array}$ & $\begin{array}{l}\text { Trend } \\
(\mathrm{cm})\end{array}$ & $\begin{array}{l}\text { Amp } \\
(\mathrm{cm} / \mathrm{yr})\end{array}$ & $\begin{array}{l}\text { Phase } \\
\text { (deg) }\end{array}$ & $\begin{array}{l}\text { Trend } \\
(\mathrm{cm})\end{array}$ & $\begin{array}{l}\text { Amp } \\
(\mathrm{cm} / \mathrm{yr})\end{array}$ & $\begin{array}{l}\text { Phase } \\
\text { (deg) }\end{array}$ \\
\hline 1 & 1.12 & 9.21 & 10.52 & 0.31 & 9.51 & 9.50 & 0.39 & 9.68 & 9.42 \\
\hline 2 & -0.95 & 7.04 & 27.87 & -2.38 & 5.80 & 23.21 & -2.45 & 5.77 & 27.30 \\
\hline 3 & 1.48 & 8.40 & 219.58 & 2.49 & 5.85 & 220.43 & 1.58 & 7.94 & 220.37 \\
\hline 4 & -0.58 & 19.18 & 26.41 & -1.61 & 15.83 & 22.35 & -1.09 & 16.38 & 25.41 \\
\hline 5 & 3.05 & 12.48 & 24.33 & 2.95 & 9.25 & 21.68 & 2.74 & 9.76 & 20.17 \\
\hline 6 & 1.03 & 15.97 & 2.28 & 1.01 & 12.58 & 2.63 & 0.27 & 13.64 & 359.07 \\
\hline 7 & 2.19 & 3.24 & 195.61 & 1.33 & 3.36 & 194.45 & 2.27 & 1.18 & 219.95 \\
\hline 8 & 4.43 & 9.69 & 236.32 & 1.44 & 12.64 & 242.67 & 2.41 & 10.46 & 239.02 \\
\hline
\end{tabular}

Table 5.5: Best fit trend, annual amplitude, and phase values for South America basins: instrument noise only.
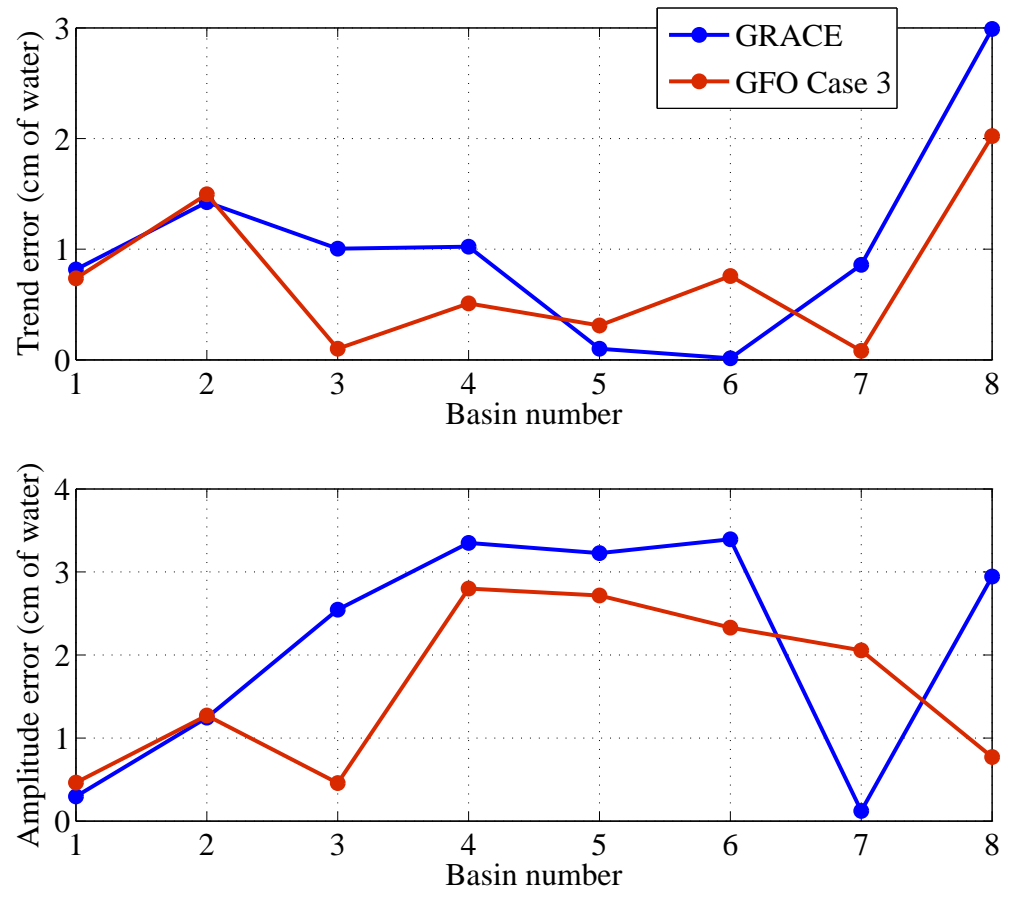

Figure 5.27: Errors in trend and amplitude estimates for each South America basin for GRACE and GFO Case 3: instrument noise only. 


\subsubsection{Greenland}

Figure 5.28 shows the RMS errors of the monthly Greenland spatial maps for the four simulated missions where only instrument errors are included in the simulations. The mean of the monthly RMS values are given in Table 5.6. The "V" shape in the time series of monthly RMS values is due to the nature of the Truth gravity signal. The Truth signal has large linear trends at small spatial scales which are not perfectly recovered by the simulation. Therefore, the level of error is dependent on the magnitude of the trend signal, which has the same "V" shape over the 25 month simulation.

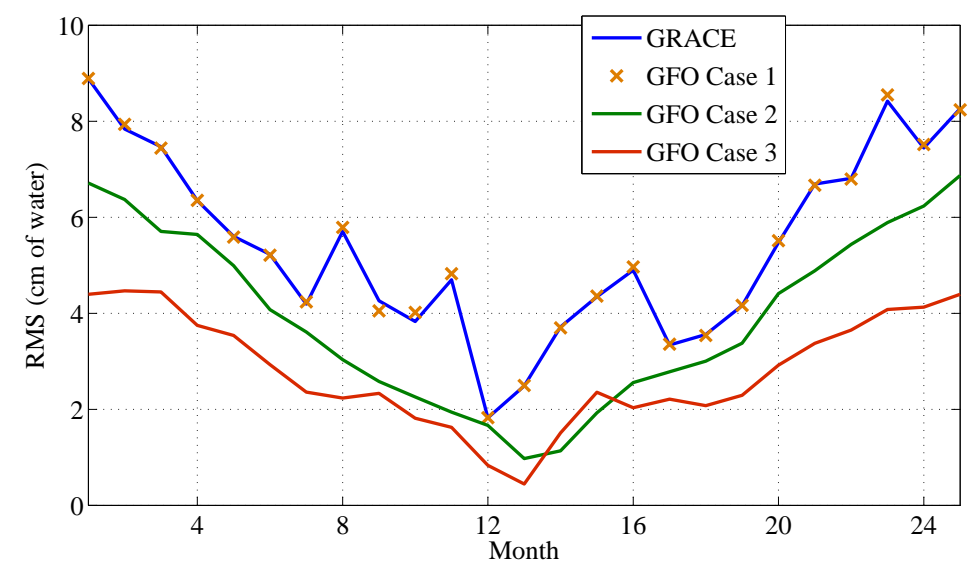

Figure 5.28: RMS errors of Greenland spatial maps for all mission configurations: instrument noise only case.

\begin{tabular}{|l|l|}
\hline $\begin{array}{l}\text { Mission } \\
\text { Configuration }\end{array}$ & $\begin{array}{l}\overline{\mathbf{R M S}} \\
\text { (cm of water) }\end{array}$ \\
\hline GRACE & 5.42 \\
\hline GFO Case 1 & 5.44 \\
\hline GFO Case 2 & 3.92 \\
\hline GFO Case 3 & 2.81 \\
\hline
\end{tabular}

Table 5.6: Mean of monthly RMS of 25 Greenland spatial plots for instrument noise only case.

As was observed for the South America simulations, the estimates for GRACE 
and GFO Case 1 have nearly the exact same level of error. There is, however, a distinct difference between the errors of GFO Case 2 and GFO Case 3 showing that the improved ranging accuracy for GFO Case 3 does provide a benefit in some cases. The reason no difference is observed between GFO Case 2 and GFO Case 3 for the South America simulations is due to the hydrologic aliasing which is common to both missions. This issue is explored further in Section 5.6. Analysis for the remainder of this section includes results for GRACE, GFO Case 2 and GFO Case 3.

The spectral density of the error maps is given in Figure 5.29. GFO Case 3 shows the lowest level of error for all sampled spatial frequencies. GFO Case 3 and GFO Case 2 show large reductions in the error at the lowest spatial scales as compared to the GRACE estimates. The error is approximately equal to the power in the signal near harmonic degree 120.

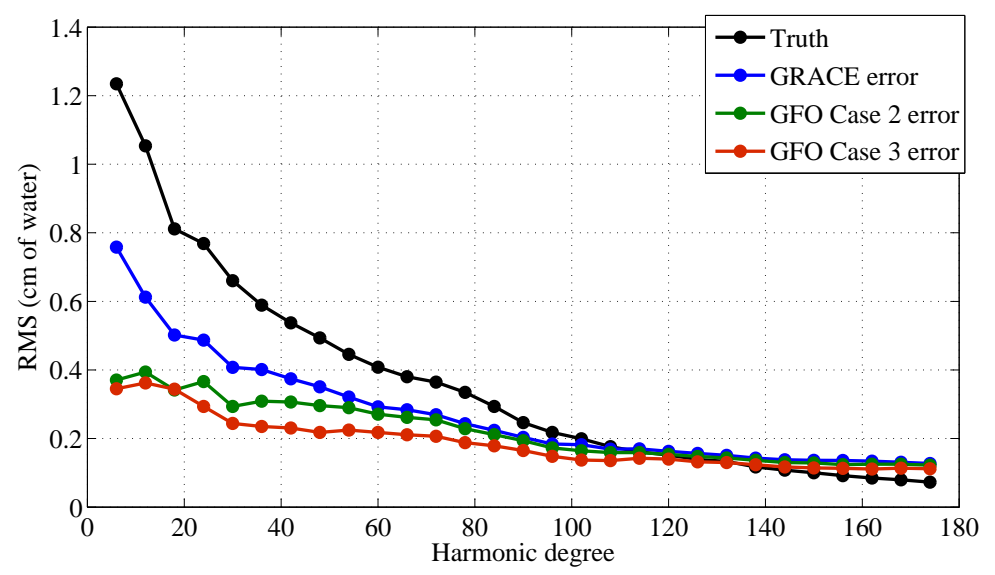

Figure 5.29: Mean spectral density of monthly spatial maps for the Truth signal and GRACE and GFO Case 3 error over Greenland: instrument noise only.

The time series of mass changes for the Greenland basins are plotted in Figure 5.30 and total mass change is in Figure 5.31. The RMS errors for the various missions over all basins are given in Table 5.7. GFO Case 3 has the lowest RMS values for nine of 
the twelve basins and for the total Greenland signal, while GRACE has the lowest RMS for only one of the basins. The time series of mass variations show that the GRACE signal tends to be much noisier those for GFO Case 2 and GFO Case 3.

Once again, a constant, trend, annual amplitude, and phase are estimated from the time series of mass variations. The values for the Truth, GRACE, and GFO Case 3 are shown in Table 5.8 and the errors of the trend and amplitude estimates are plotted in Figure 5.32. Some improvement in the estimates of these time change variables is observed for GFO Case 3 as compared to GRACE. 

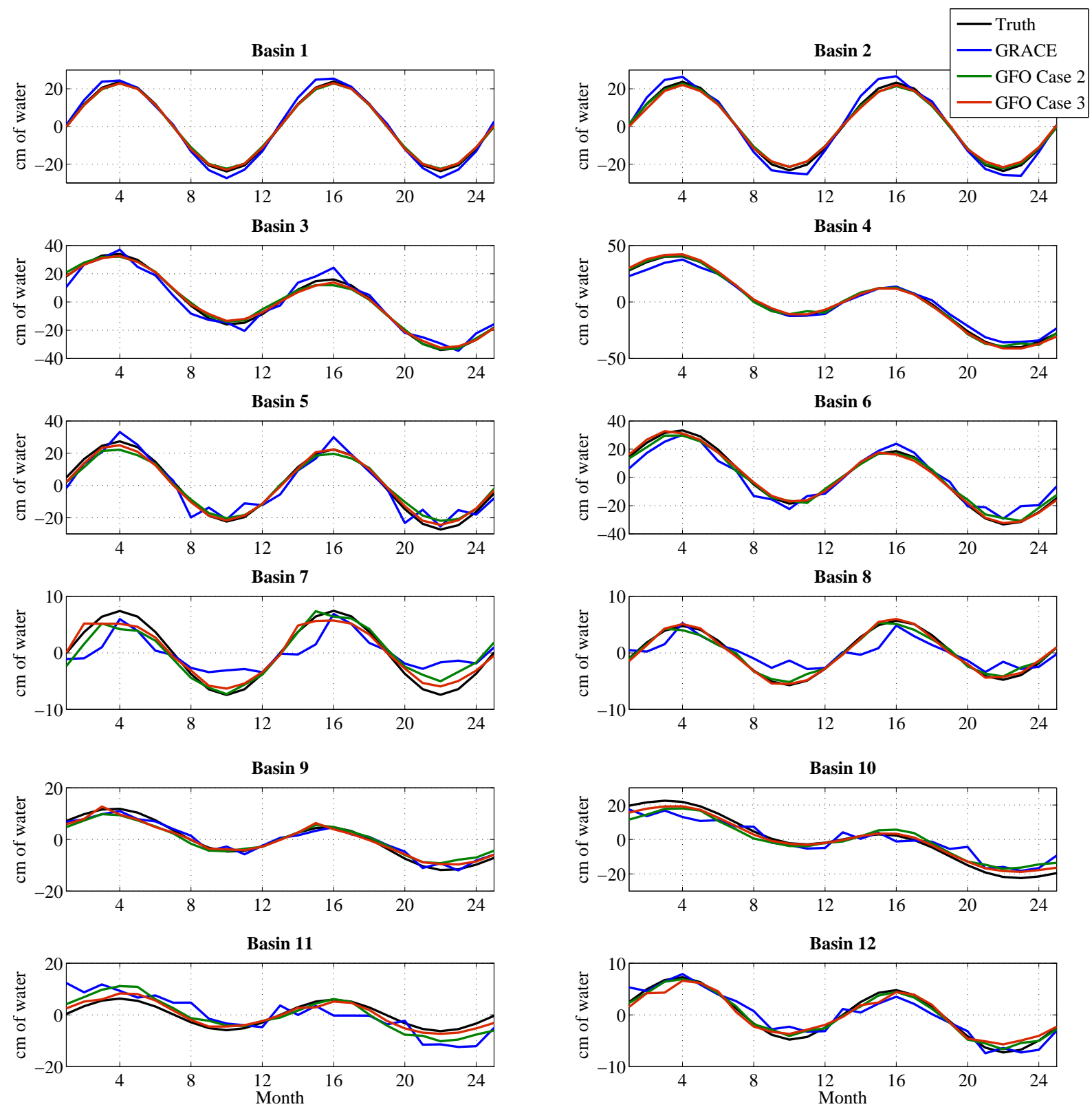

Figure 5.30: Greenland basin mass variations time series for different mission configurations: instrument noise only. 


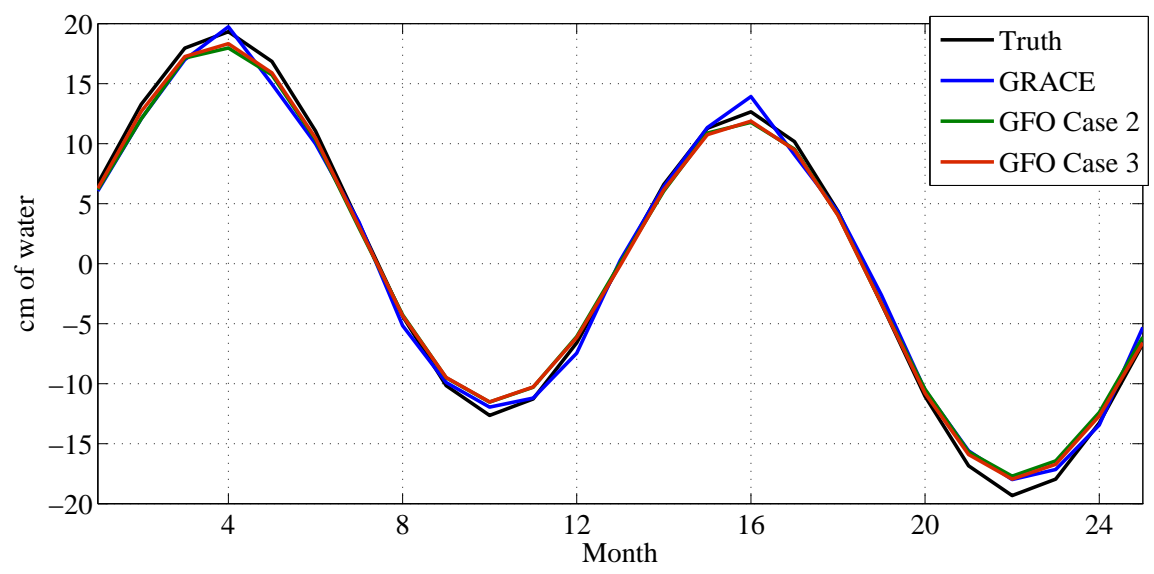

Figure 5.31: Total Greenland variation time series for different mission configurations: instrument noise only.

\begin{tabular}{|l||l||l|l|l|}
\hline Basin & $\begin{array}{l}\text { Truth } \\
\text { signal RMS } \\
\text { (cm of water) }\end{array}$ & $\begin{array}{l}\text { GRACE } \\
\text { error RMS } \\
\text { (cm of water) }\end{array}$ & $\begin{array}{l}\text { GFO Case 2 } \\
\text { error RMS } \\
\text { (cm of water })\end{array}$ & $\begin{array}{l}\text { GFO Case 3 } \\
\text { error RMS } \\
\text { (cm of water })\end{array}$ \\
\hline 1 & 16.513 & 2.087 & 0.793 & 0.592 \\
\hline 2 & 16.306 & 2.767 & 1.045 & 1.397 \\
\hline 3 & 20.708 & 3.904 & 1.803 & 1.612 \\
\hline 4 & 24.937 & 3.293 & 1.653 & 1.426 \\
\hline 5 & 17.647 & 5.149 & 3.040 & 1.781 \\
\hline 6 & 20.325 & 5.328 & 2.226 & 1.459 \\
\hline 7 & 5.145 & 3.090 & 1.622 & 1.105 \\
\hline 8 & 3.628 & 1.961 & 0.605 & 0.347 \\
\hline 9 & 7.241 & 1.419 & 1.846 & 1.426 \\
\hline 10 & 14.295 & 5.124 & 4.152 & 2.286 \\
\hline 11 & 4.258 & 5.166 & 3.231 & 1.592 \\
\hline 12 & 4.496 & 1.397 & 0.600 & 1.000 \\
\hline Total & 11.959 & 0.875 & 0.861 & 0.712 \\
\hline
\end{tabular}

Table 5.7: RMS of errors for Greenland basin mass estimates for different mission configurations shown in Figure 5.30: instrument noise only. 


\begin{tabular}{|c|c|c|c|c|c|c|c|c|c|}
\hline \multirow[t]{2}{*}{ Basin } & \multicolumn{3}{|c|}{ Truth } & \multicolumn{3}{|c|}{ GRACE } & \multicolumn{3}{|c|}{ GFO Case 3} \\
\hline & $\begin{array}{l}\text { Trend } \\
(\mathrm{cm})\end{array}$ & $\begin{array}{l}\text { Amp } \\
(\mathrm{cm} / \mathrm{yr})\end{array}$ & $\begin{array}{l}\text { Phase } \\
\text { (deg) }\end{array}$ & $\begin{array}{l}\text { Trend } \\
(\mathrm{cm})\end{array}$ & $\begin{array}{l}\text { Amp } \\
(\mathrm{cm} / \mathrm{yr})\end{array}$ & $\begin{array}{l}\text { Phase } \\
\text { (deg) }\end{array}$ & $\begin{array}{l}\text { Trend } \\
(\mathrm{cm})\end{array}$ & $\begin{array}{l}\text { Amp } \\
(\mathrm{cm} / \mathrm{yr})\end{array}$ & $\begin{array}{l}\text { Phase } \\
\text { (deg) }\end{array}$ \\
\hline 1 & 0.13 & 23.88 & 0.00 & 0.50 & 26.28 & 1.84 & 0.24 & 23.12 & 0.35 \\
\hline 2 & -0.39 & 23.41 & 0.00 & -0.39 & 26.56 & 1.58 & 0.13 & 21.78 & -0.34 \\
\hline 3 & -18.07 & 20.44 & 0.00 & -13.84 & 22.37 & 2.01 & -18.89 & 18.10 & -0.66 \\
\hline 4 & -27.89 & 19.35 & 0.00 & -23.41 & 18.55 & -4.28 & -30.27 & 18.95 & 0.53 \\
\hline 5 & -4.96 & 23.61 & 0.00 & -3.59 & 22.79 & -1.24 & -2.65 & 22.79 & 0.25 \\
\hline 6 & -14.70 & 22.24 & 0.00 & -6.83 & 22.62 & 2.30 & -15.66 & 20.81 & 1.33 \\
\hline 7 & 0.04 & 7.44 & 0.00 & 1.18 & 3.90 & -6.90 & 0.05 & 6.18 & 2.65 \\
\hline 8 & 0.97 & 5.48 & 0.00 & -0.23 & 2.94 & -10.10 & 1.14 & 5.53 & 0.92 \\
\hline 9 & -7.16 & 6.47 & 0.00 & -6.22 & 5.73 & -3.80 & -5.82 & 5.76 & 3.43 \\
\hline 10 & -19.58 & 7.12 & 0.00 & -13.25 & 5.52 & -3.78 & -15.89 & 6.95 & 0.10 \\
\hline 11 & -0.36 & 6.03 & 0.00 & -8.59 & 4.27 & -2.92 & -2.91 & 5.62 & -1.45 \\
\hline 12 & -2.48 & 5.40 & 0.00 & -4.11 & 3.99 & -3.52 & -1.99 & 4.52 & -1.33 \\
\hline
\end{tabular}

Table 5.8: Best fit trend, annual amplitude, and phase values for Greenland basins: instrument noise only.
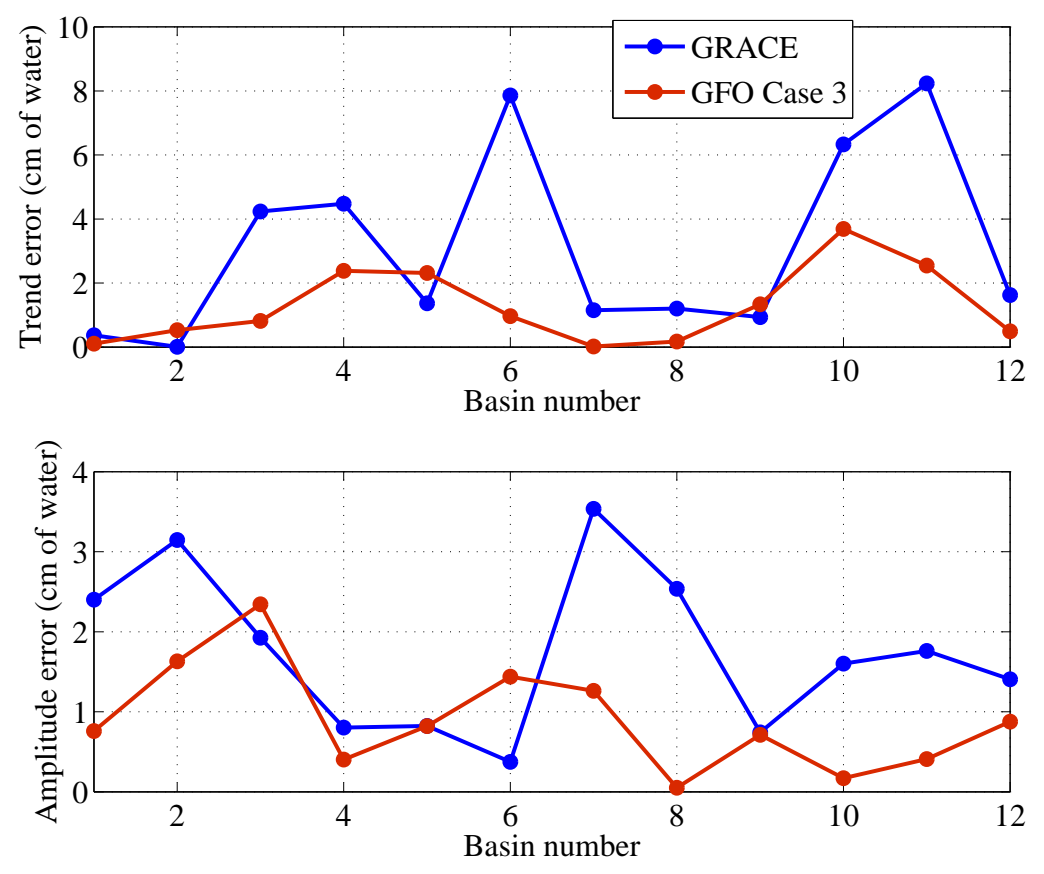

Figure 5.32: Errors in trend and amplitude estimates for each Greenland basin for GRACE and GFO Case 3: instrument noise only. 


\subsubsection{Spatial Resolution Study}

The simulation results in this section aim to investigate the best attainable spatial resolution of the gravity estimates for each mission. As discussed earlier, the errors caused by temporal aliasing are largely dependent on location, so it is not practical to discuss the spatial resolution of a mission for regional gravity estimation in the presence of temporal aliasing errors. The simulated gravity estimates given here are for a Truth signal of $100 \mathrm{~cm}$ of water over a $1^{\circ} \times 1^{\circ}$ block at the equator in the Amazon basin, for instrument errors only. Simulations are performed for 31 days of data for the four considered mission configurations.

The resulting gravity estimates for all cases are shown in Figure 5.33. As before, the results for GRACE and GFO Case 1 are indistinguishable from each other. In addition to the high signal over the $1^{\circ} \times 1^{\circ}$ block at the equator, there are additional locations where the gravity signal is high. These errors in the estimates are due to the accelerometer error. A clear difference is observable between the GFO Case 2 and GFO Case 3 estimates. Due to the higher satellite-to-satellite ranging noise for GFO Case 2, the gravity field cannot be estimated to as high of a spatial resolution as for GFO Case 3 equipped with the laser. However, GFO Case 2 clearly provides a much less noisy solution than GRACE and GFO Case 1.

The plotted data in Figure 5.34 shows the recovered mass for each mission within a series of concentric squares of increasing size. The box sizes and corresponding surface areas at which the simulated gravity estimates are sampled are given in Table 5.9. The true amount of mass within a $1^{\circ} \times 1^{\circ}$ block at the equator is $12.39 \mathrm{GT}$ and is included in the figure. GFO Case 3 performs significantly better than the other missions as it measures nearly all of the mass within a $3^{\circ} \times 3^{\circ}$ block. GFO Case 2 performs better than 
the other two missions by recovering more mass within each of the sampled boxes and all of the approximately all of the mass for a $7^{\circ} \times 7^{\circ}$ block. The GRACE and GFO Case 1 missions do not recover all of the mass for any size block. Again, this is due to the effect of the accelerometer errors on the solution. For a different month with a slightly different groundtrack it would be possible for the GRACE and GFO Case 1 mission to overestimate the mass within the block, depending on the spatial characteristics of the accelerometer errors. 


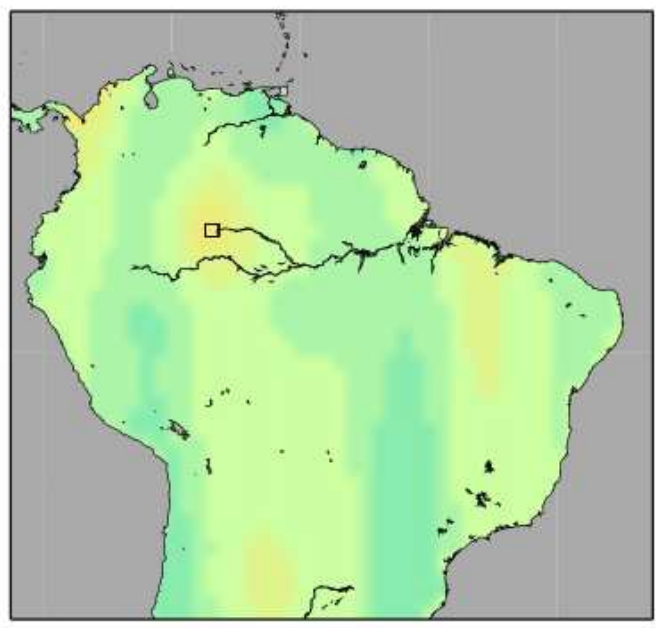

(a) GRACE

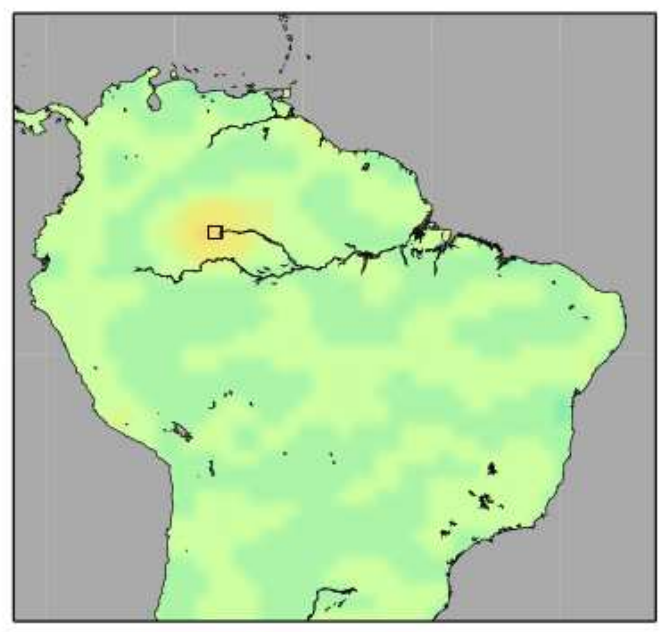

(c) GFO Case 2

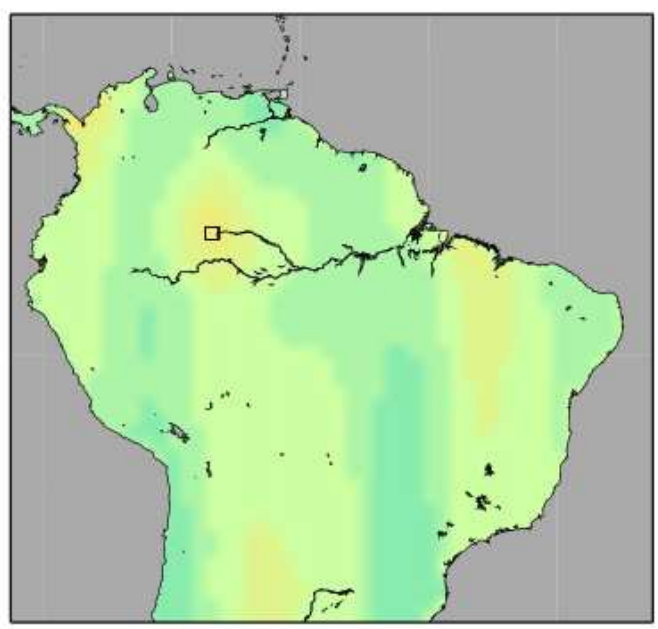

(b) GFO Case 1

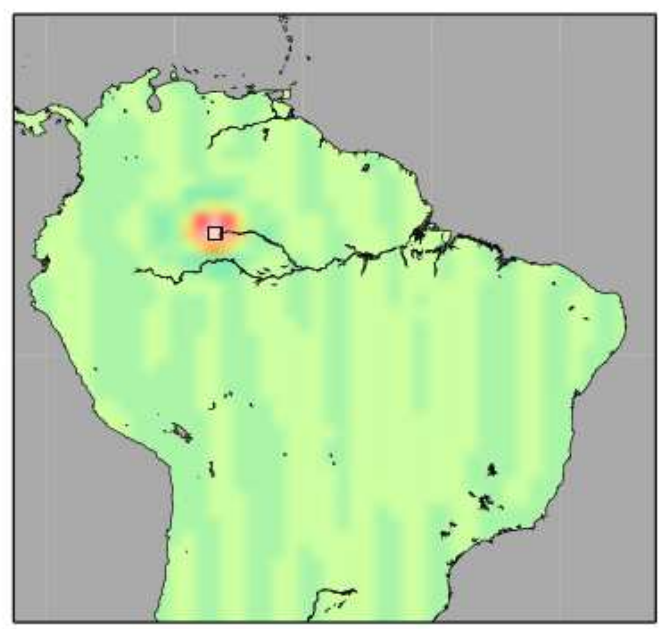

(d) GFO Case 3

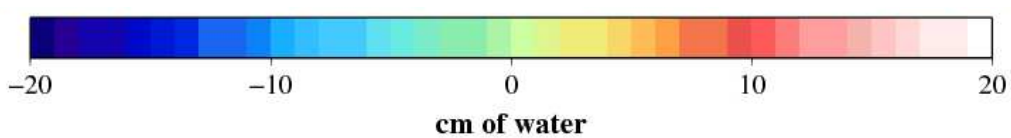

Figure 5.33: Gravity estimates for $1^{\circ} \times 1^{\circ}$ block of $100 \mathrm{~cm}$ of water for different mission configutations. The location of the Truth signal is shown on each plot. 


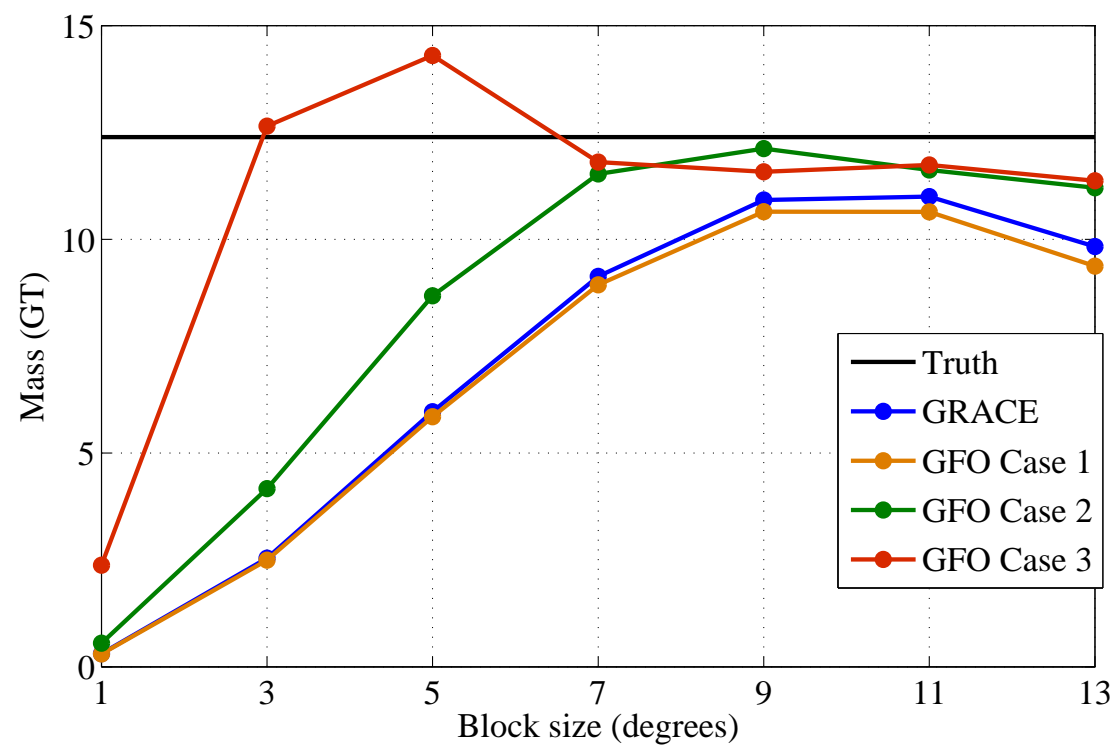

Figure 5.34: Mass recovered within a given size block for different mission configurations: instrument noise only.

\begin{tabular}{|l|l|}
\hline $\begin{array}{l}\text { Box size } \\
(\text { degrees })\end{array}$ & $\begin{array}{l}\text { Surface } \\
\text { Area }\left(\mathrm{km}^{2}\right)\end{array}$ \\
\hline $1^{\circ} \times 1^{\circ}$ & $1.239 \times 10^{4}$ \\
\hline $3^{\circ} \times 3^{\circ}$ & $1.115 \times 10^{5}$ \\
\hline $5^{\circ} \times 5^{\circ}$ & $3.096 \times 10^{5}$ \\
\hline $7^{\circ} \times 7^{\circ}$ & $6.068 \times 10^{5}$ \\
\hline $9^{\circ} \times 9^{\circ}$ & $1.002 \times 10^{6}$ \\
\hline $11^{\circ} \times 11^{\circ}$ & $1.497 \times 10^{6}$ \\
\hline $13^{\circ} \times 13^{\circ}$ & $2.089 \times 10^{6}$ \\
\hline
\end{tabular}

Table 5.9: Sampled box sizes and surface areas. 


\subsection{Simulated Gravity Estimates: All Error Sources}

The simulated gravity results presented in this section contain all major error sources which affect the estimation of time variable gravity. The only change in the simulations in this section from the previous section is the inclusion of the temporal aliasing errors caused by the errors in the modeled atmospheric, oceanographic, and tidal signals.

\subsubsection{South America}

The spatial maps of the South America gravity estimates for GRACE, GFO Case

1, GFO Case 2, and GFO Case 3 are shown in Figures 5.35, 5.36, 5.37, and 5.38 respectively. Again, the GRACE and GFO Case 1 results look identical to each other, as do the GFO Case 2 and GFO Case 3 estimates. 

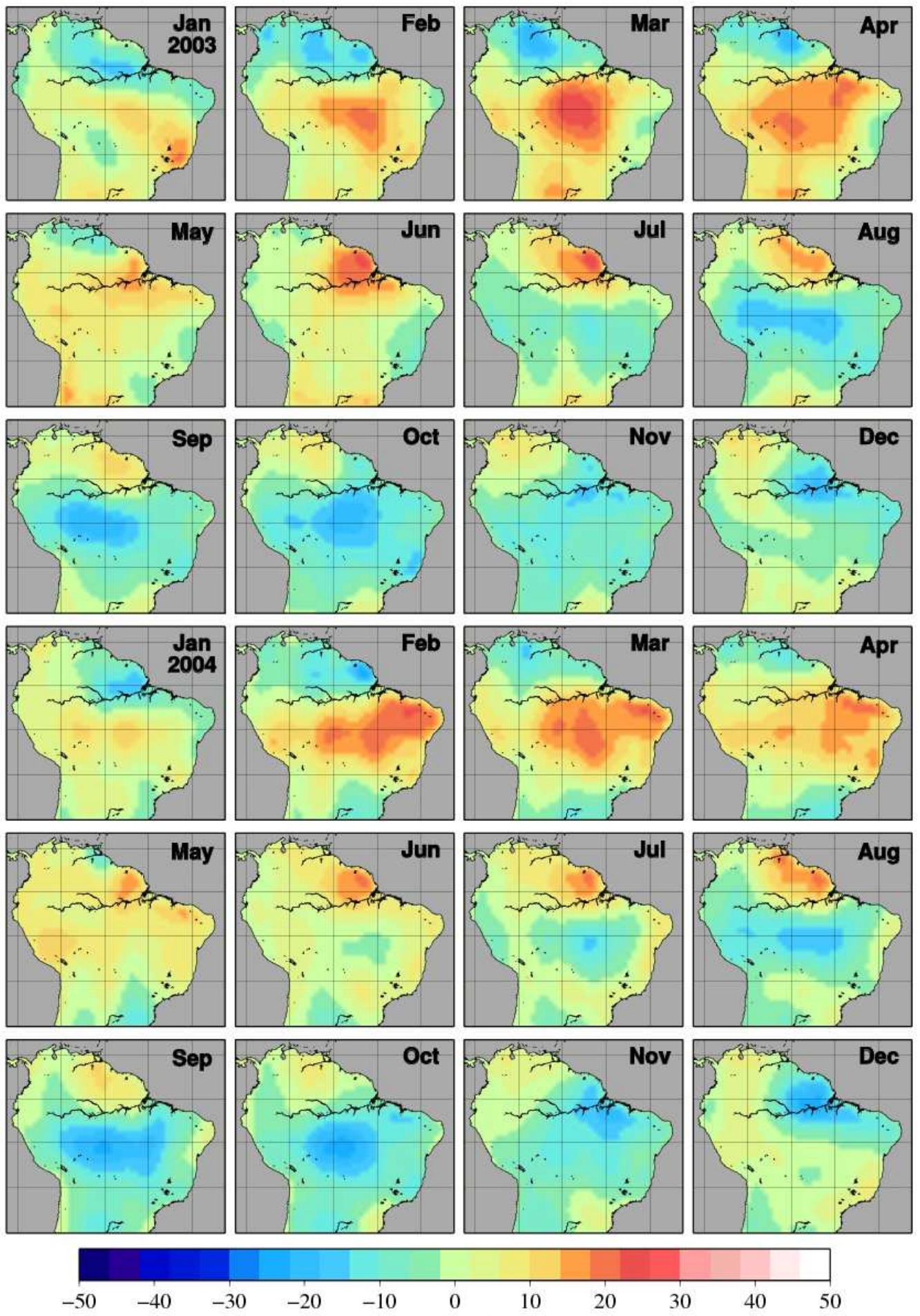

cm of water

Figure 5.35: South America gravity estimates for GRACE (480 km altitude / K-band / Accelerometer): all error sources. 

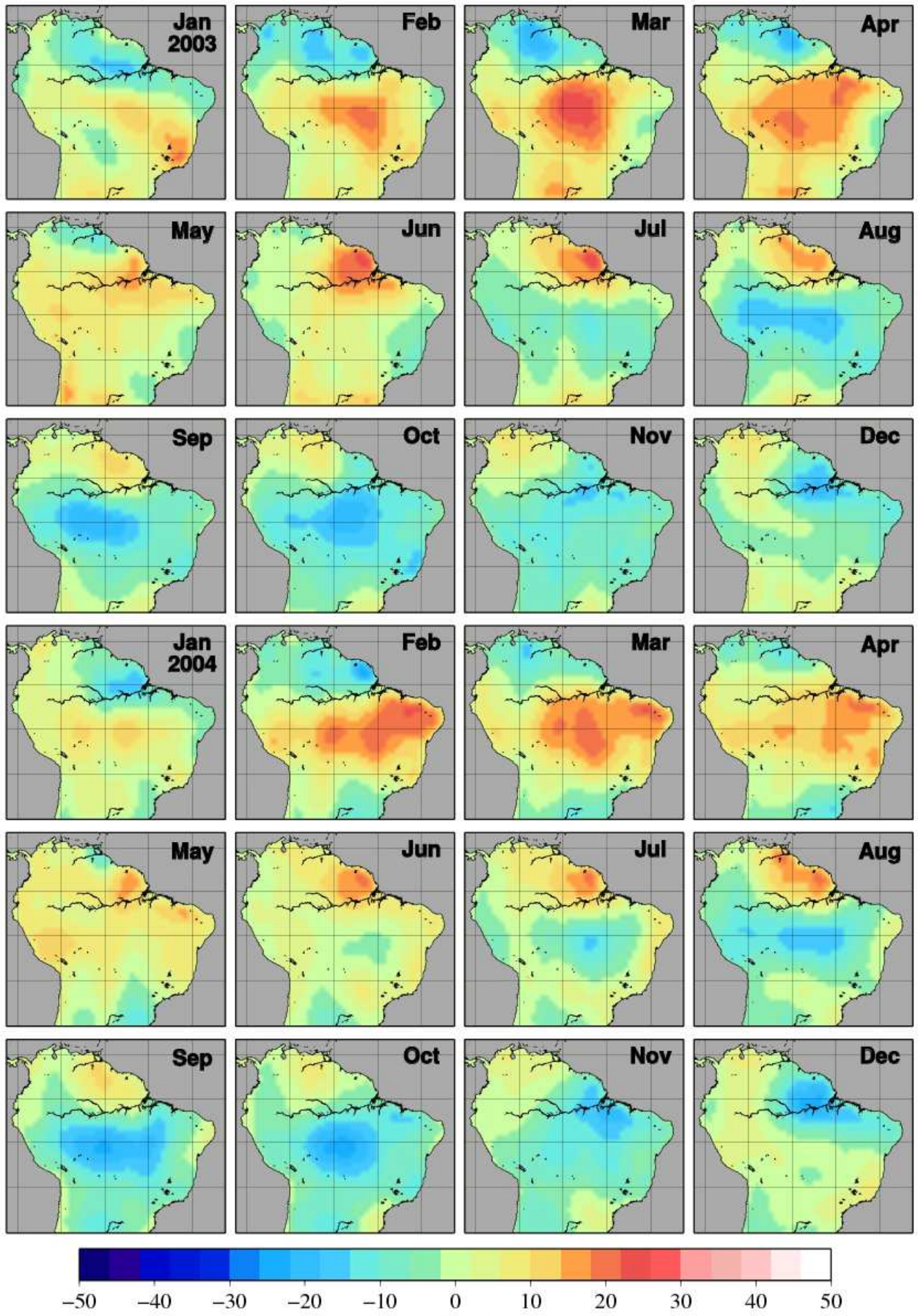

cm of water

Figure 5.36: South America gravity estimates for GFO Case 1 (480 km altitude / Laser / Accelerometer): all error sources. 

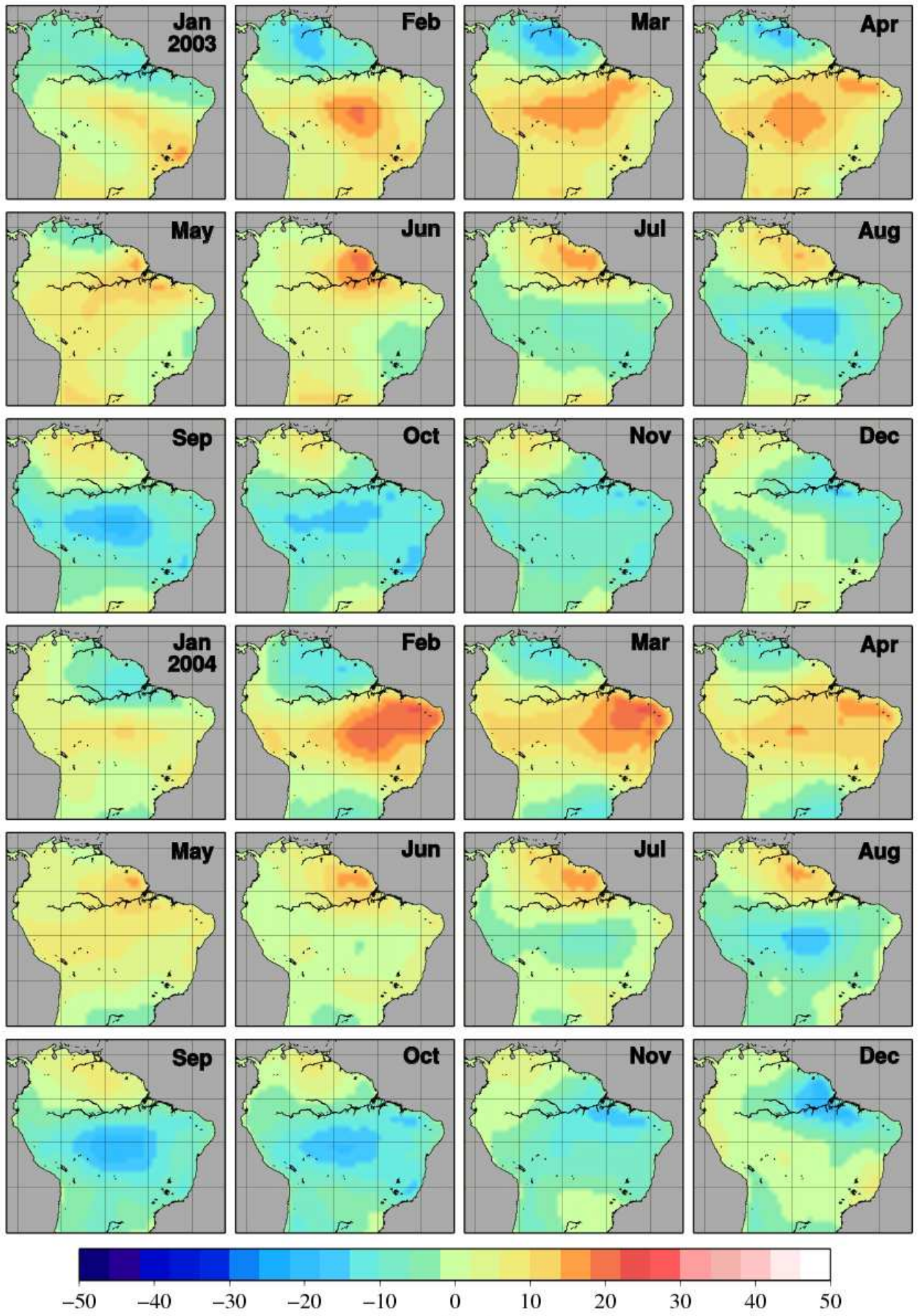

cm of water

Figure 5.37: South America gravity estimates for GFO Case 2 (250 km altitude / Kband / GRS): all error sources. 

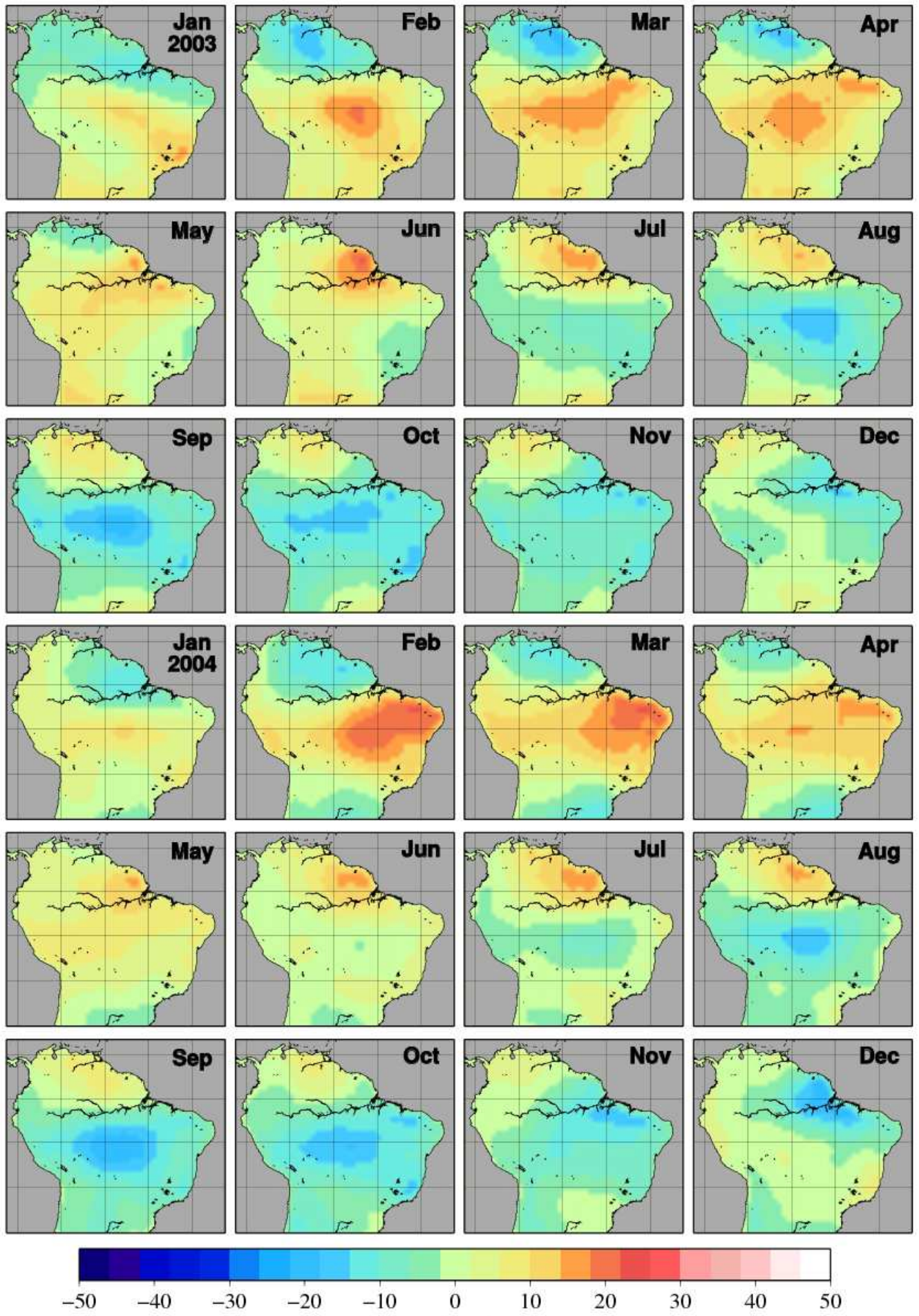

cm of water

Figure 5.38: South America gravity estimates for GFO Case 3 (250 km altitude / Laser / GRS): all error sources. 


\subsubsection{Greenland}

Simulated gravity estimates for Greenland for all errors sources for the four mission setups are shown in Figures 5.39, 5.40, 5.41, and 5.42. As was observed for the South America all errors case, the Greenland GRACE and GFO Case 1 estimates appear to be the same, as do the GFO Case 2 and GFO Case 3 estimates. 

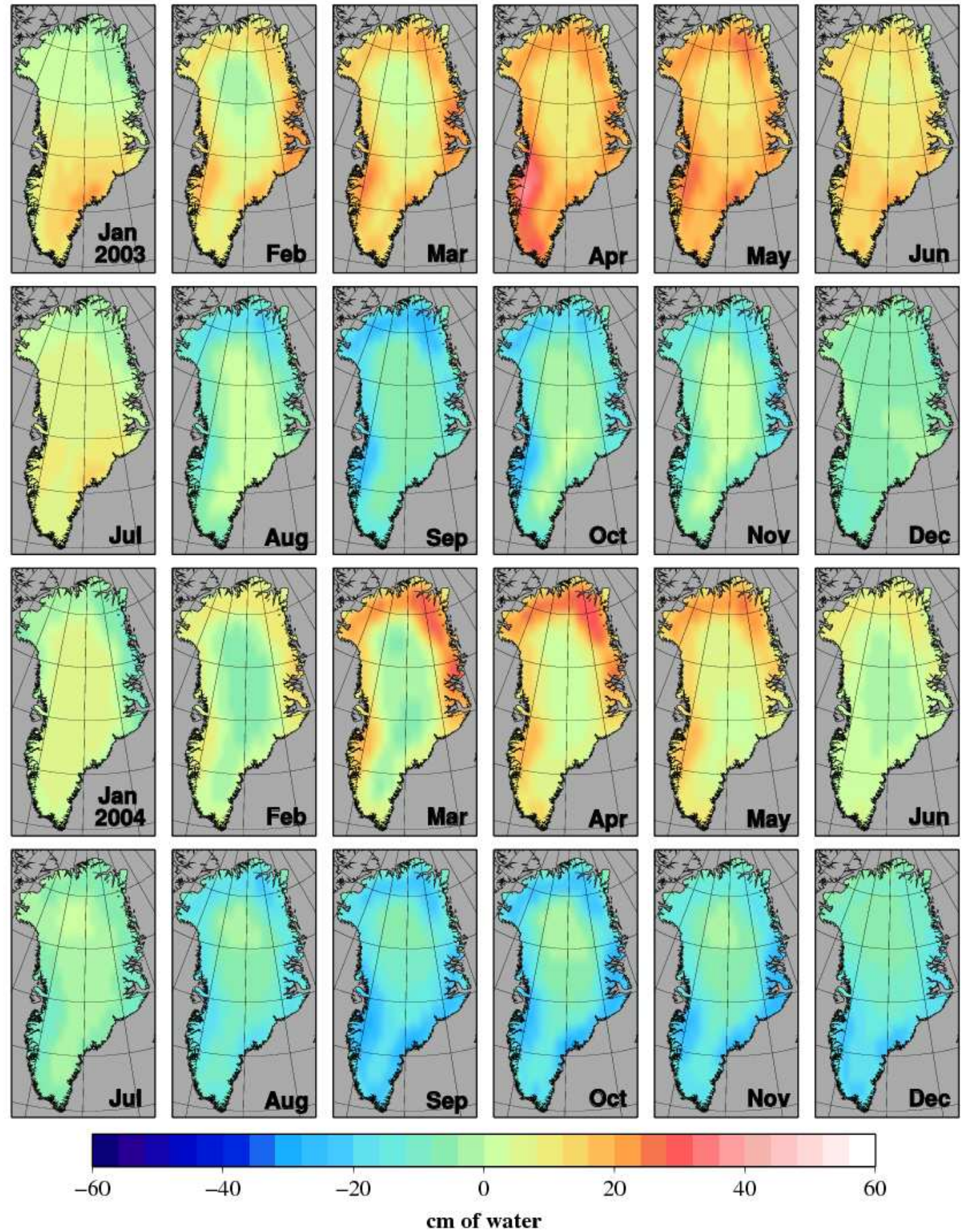

Figure 5.39: Greenland gravity estimates for GRACE (480 km altitude / K-band / Accelerometer): all error sources. 

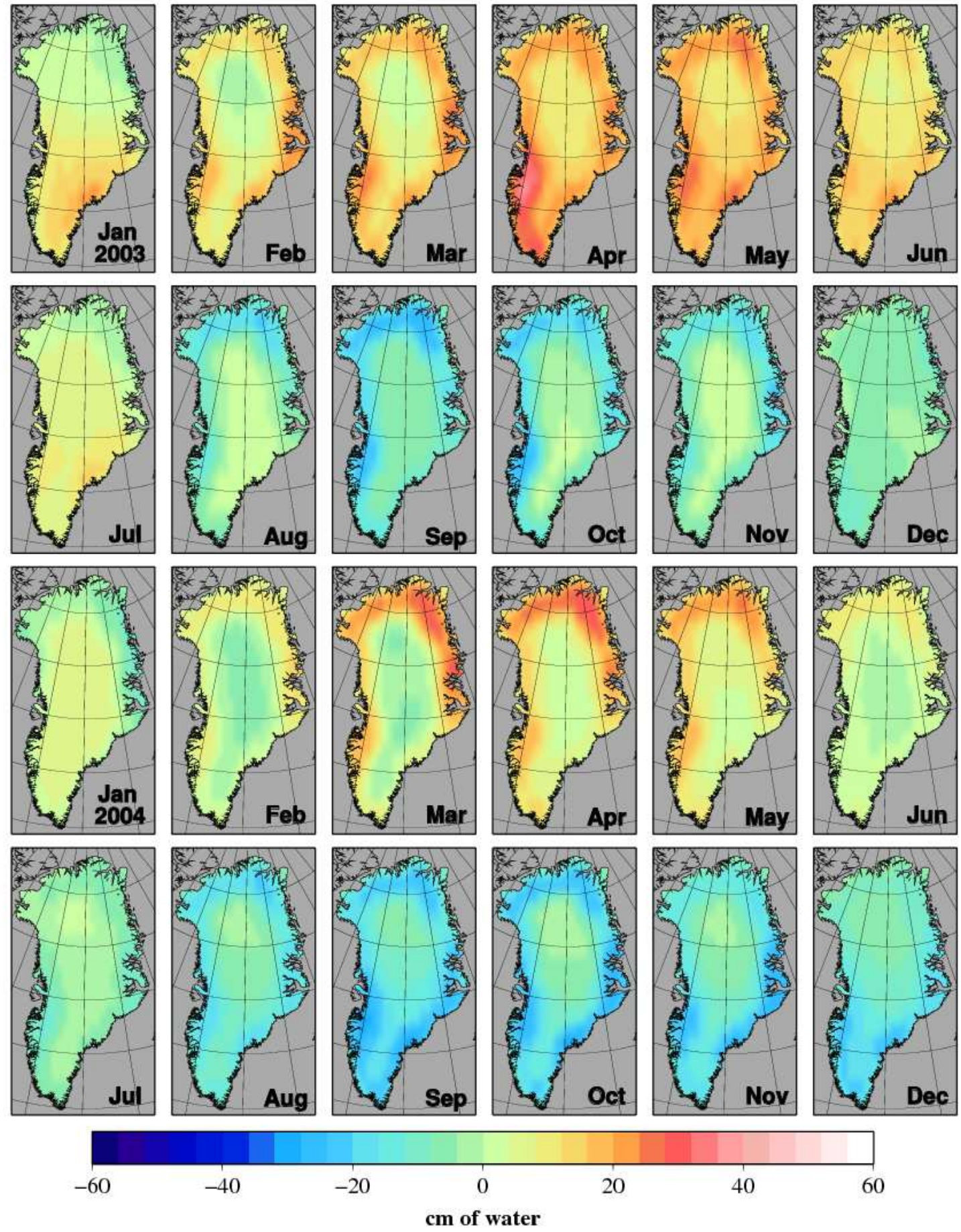

Figure 5.40: Greenland gravity estimates for GFO Case 1 (480 km altitude / Laser / Accelerometer): all error sources. 

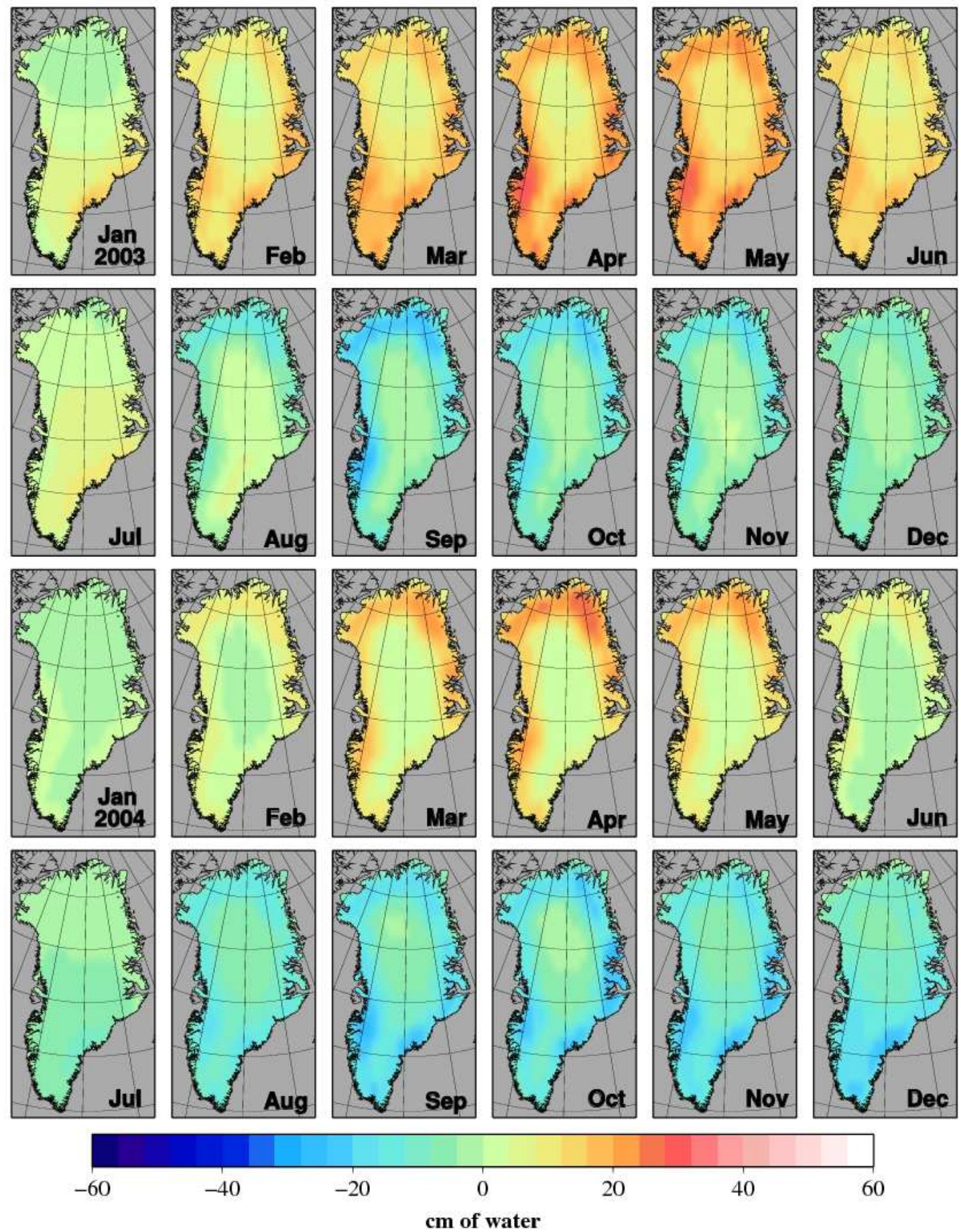

Figure 5.41: Greenland gravity estimates for GFO Case 2 (250 km altitude / K-band / GRS): all error sources. 

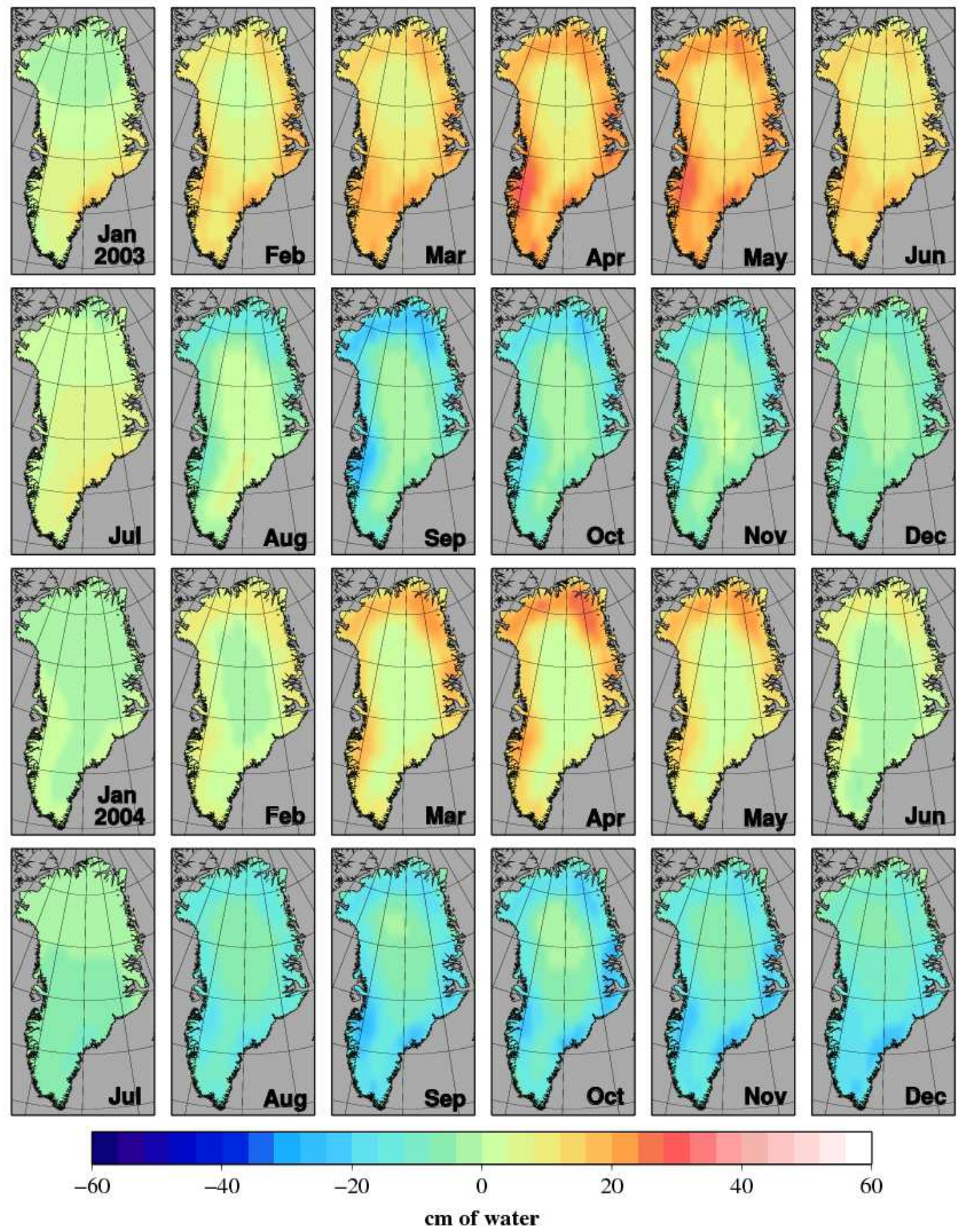

Figure 5.42: Greenland gravity estimates for GFO Case 3 (250 km altitude / Laser / GRS): all error sources. 


\subsubsection{Comparison of Mission Configurations}

\subsubsection{South America}

The results are analyzed using the same statistical tools as before. Figure 5.43 shows the monthly error RMS values of the South America spatial maps for the four simulated missions where all error sources have been included. As was seen before, there is no noticeable difference between the GRACE and GFO Case 1 performance, and between the GFO Case 2 and GFO Case 3 performance. There is also a very minor performance difference between GRACE and GFO Case 3 with a difference of mean error RMS of $\sim 0.1 \mathrm{~cm}$ of water as shown in Table 5.10. The remaining analysis in this subsection only considers the results for GRACE and GFO Case 3.

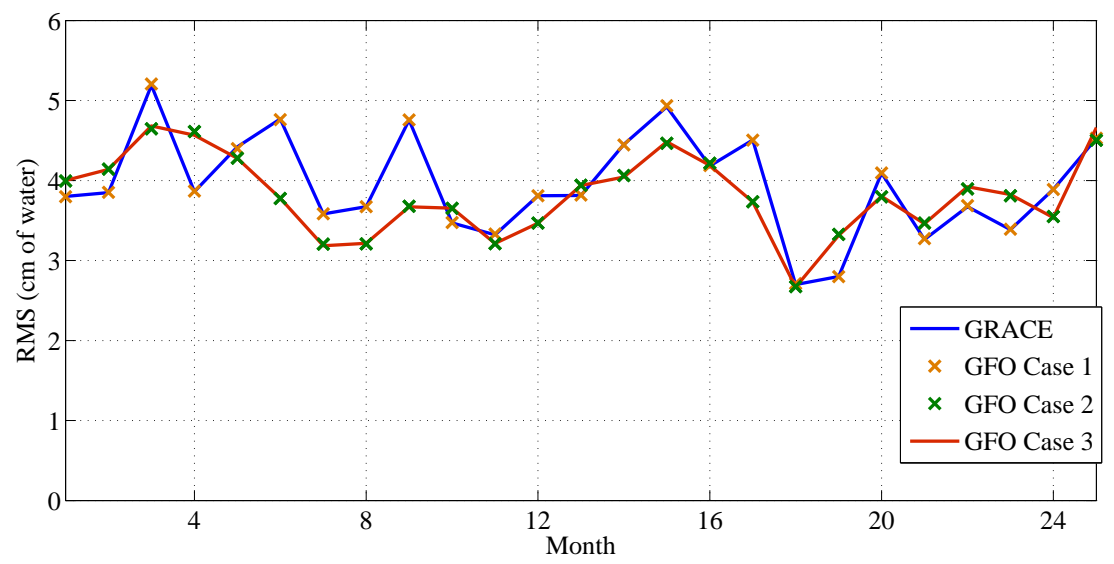

Figure 5.43: RMS errors of South America spatial maps for all mission configurations: all error sources.

\begin{tabular}{|l|l|}
\hline $\begin{array}{l}\text { Mission } \\
\text { Configuration }\end{array}$ & $\begin{array}{l}\overline{\mathbf{R M S}} \\
\text { (cm of water) }\end{array}$ \\
\hline GRACE & 3.95 \\
\hline GFO Case 1 & 3.95 \\
\hline GFO Case 2 & 3.81 \\
\hline GFO Case 3 & 3.82 \\
\hline
\end{tabular}

Table 5.10: Mean of monthly RMS of 25 South America spatial plots for all errors case. 
The spectral density of the error spatial maps shown in Figure 5.44 show that the spatial characteristics of the errors for the different missions are nearly identical.

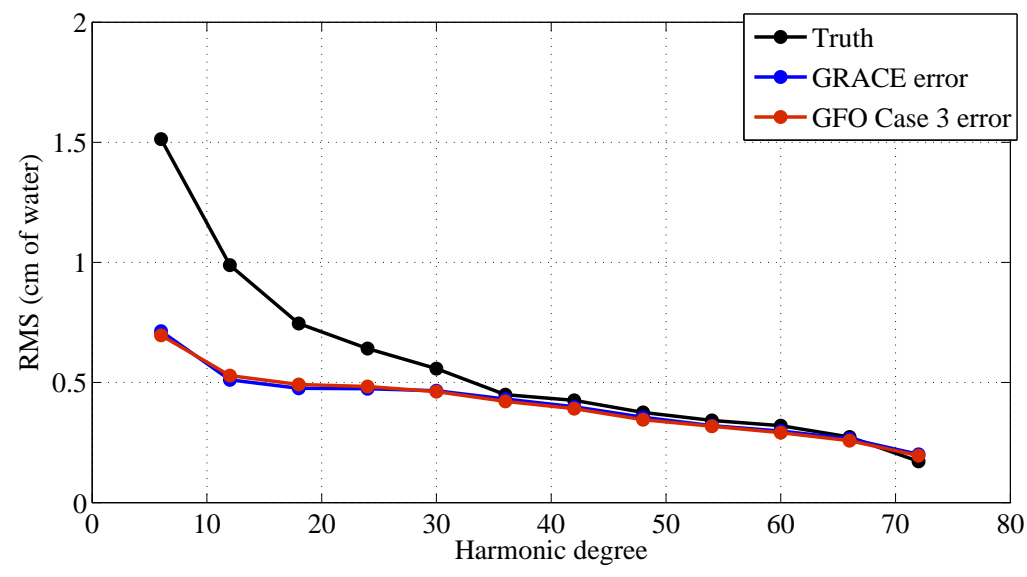

Figure 5.44: Mean spectral density of monthly spatial maps for the Truth signal and GRACE and GFO Case 3 error over South America: all error sources.

The time series of mass variations for the South America basins are shown in Figure 5.45 and the corresponding error RMS values are in Table 5.11. As expected from the previous analaysis, there is not much of a difference in the results between the simulated missions. GFO Case 3 does have a smaller error RMS for six of the eight basins than GRACE but the differences are quite small.

Table 5.12 shows the trend, annual amplitude, and phase of the Truth, GRACE, and GFO Case 3 mass variation time series for each basin. The error in trend and annual amplitude are plotted in Figure 5.46. GFO Case 3 estimates do provide a noticeable improvement in the trend estimates for some of the basins, while neither mission consistently provides better estimates of the annual amplitude. 

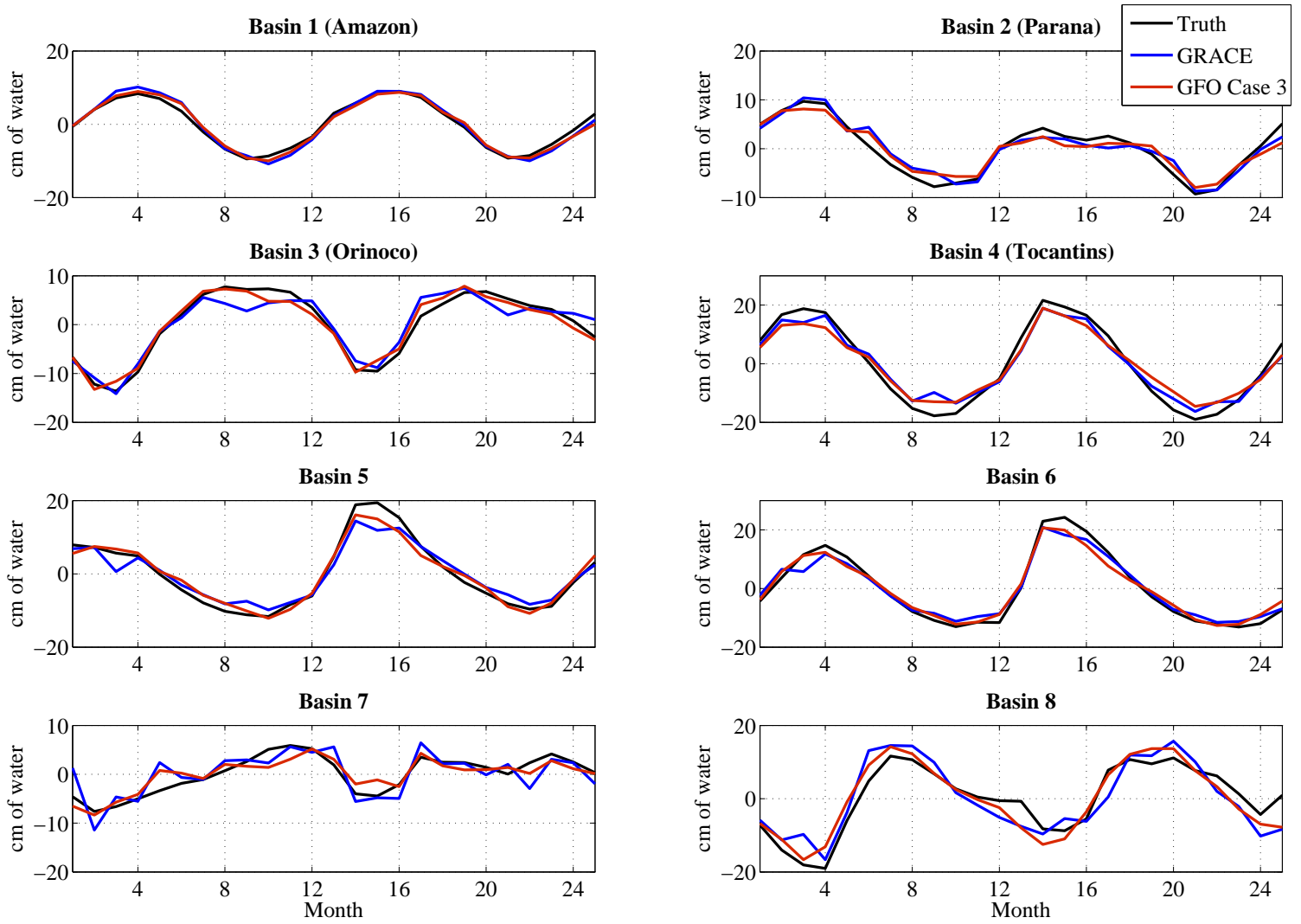

Figure 5.45: South America basin mass variations time series for different mission configurations: all error sources.

\begin{tabular}{|l||l||l|l|}
\hline Basin & $\begin{array}{l}\text { Truth } \\
\text { signal RMS } \\
\text { (cm of water) }\end{array}$ & $\begin{array}{l}\text { GRACE } \\
\text { error RMS } \\
\text { (cm of water) }\end{array}$ & $\begin{array}{l}\text { GFO Case 3 } \\
\text { error RMS } \\
\text { (cm of water) }\end{array}$ \\
\hline 1 & 6.222 & 1.243 & 1.042 \\
\hline 2 & 5.459 & 1.617 & 1.628 \\
\hline 3 & 6.705 & 2.106 & 1.267 \\
\hline 4 & 13.609 & 3.177 & 3.589 \\
\hline 5 & 9.076 & 2.587 & 1.880 \\
\hline 6 & 11.844 & 2.438 & 2.310 \\
\hline 7 & 3.790 & 2.642 & 1.809 \\
\hline 8 & 8.907 & 4.546 & 3.577 \\
\hline
\end{tabular}

Table 5.11: RMS of errors for South America basin mass estimates for different mission configurations shown in Figure 5.45: all error sources. 


\begin{tabular}{|c|c|c|c|c|c|c|c|c|c|}
\hline \multirow[t]{2}{*}{ Basin } & \multicolumn{3}{|c|}{ Truth } & \multicolumn{3}{|c|}{ GRACE } & \multicolumn{3}{|c|}{ GFO Case 3} \\
\hline & $\begin{array}{l}\text { Trend } \\
(\mathrm{cm})\end{array}$ & $\begin{array}{l}\text { Amp } \\
(\mathrm{cm} / \mathrm{yr})\end{array}$ & $\begin{array}{l}\text { Phase } \\
\text { (deg) }\end{array}$ & $\begin{array}{l}\text { Trend } \\
(\mathrm{cm})\end{array}$ & $\begin{array}{l}\text { Amp } \\
(\mathrm{cm} / \mathrm{yr})\end{array}$ & $\begin{array}{l}\text { Phase } \\
\text { (deg) }\end{array}$ & $\begin{array}{l}\text { Trend } \\
(\mathrm{cm})\end{array}$ & $\begin{array}{l}\text { Amp } \\
(\mathrm{cm} / \mathrm{yr})\end{array}$ & $\begin{array}{l}\text { Phase } \\
\text { (deg) }\end{array}$ \\
\hline 1 & 1.12 & 9.21 & 10.52 & 0.47 & 10.07 & 4.68 & 0.37 & 9.36 & 3.94 \\
\hline 2 & -0.95 & 7.04 & 27.87 & -2.16 & 5.69 & 19.40 & -2.12 & 5.02 & 21.65 \\
\hline 3 & 1.48 & 8.40 & 219.58 & 3.32 & 5.90 & 228.08 & 1.77 & 7.71 & 229.94 \\
\hline 4 & -0.58 & 19.18 & 26.41 & -1.73 & 15.10 & 22.86 & -0.35 & 14.51 & 22.41 \\
\hline 5 & 3.05 & 12.48 & 24.33 & 2.59 & 9.53 & 19.27 & 2.41 & 11.32 & 22.04 \\
\hline 6 & 1.03 & 15.97 & 2.28 & 0.70 & 13.24 & 3.28 & 0.57 & 13.65 & 4.72 \\
\hline 7 & 2.19 & 3.24 & 195.61 & 0.66 & 2.96 & 204.60 & 2.25 & 1.92 & 218.90 \\
\hline 8 & 4.43 & 9.69 & 236.32 & 0.23 & 12.30 & 245.63 & 1.45 & 12.13 & 247.55 \\
\hline
\end{tabular}

Table 5.12: Best fit trend, annual amplitude, and phase values for South America basins: all error sources.
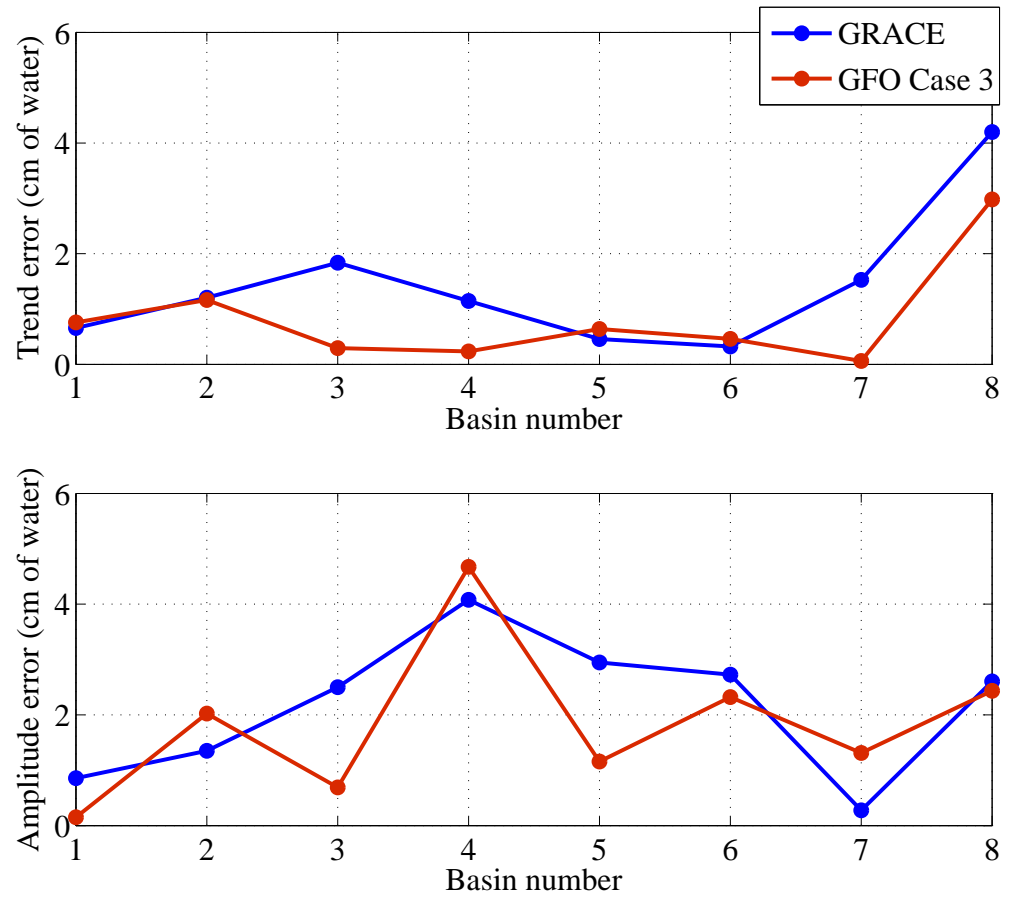

Figure 5.46: Errors in trend and amplitude estimates for each South America basin for GRACE and GFO Case 3: all error sources. 


\subsubsection{Greenland}

Monthly error RMS values for the simulated Greenland gravity estimates for the four missions where all error sources are included is given in Figure 5.47. The mean of the RMS values are given in Table 5.13. As with the South America all errors simulations, the performance of GRACE and GFO Case 1 is the same, and the performance of GFO Case 2 and GFO Case 3 is the same. There is a noticeable difference between the GRACE and GFO Case 3 performance which is most pronounced for the middle months of the simulation, when the size of the Truth signal is largest. This difference is due to the difference in the temporal aliasing errors which are discussed further in Section 5.6.

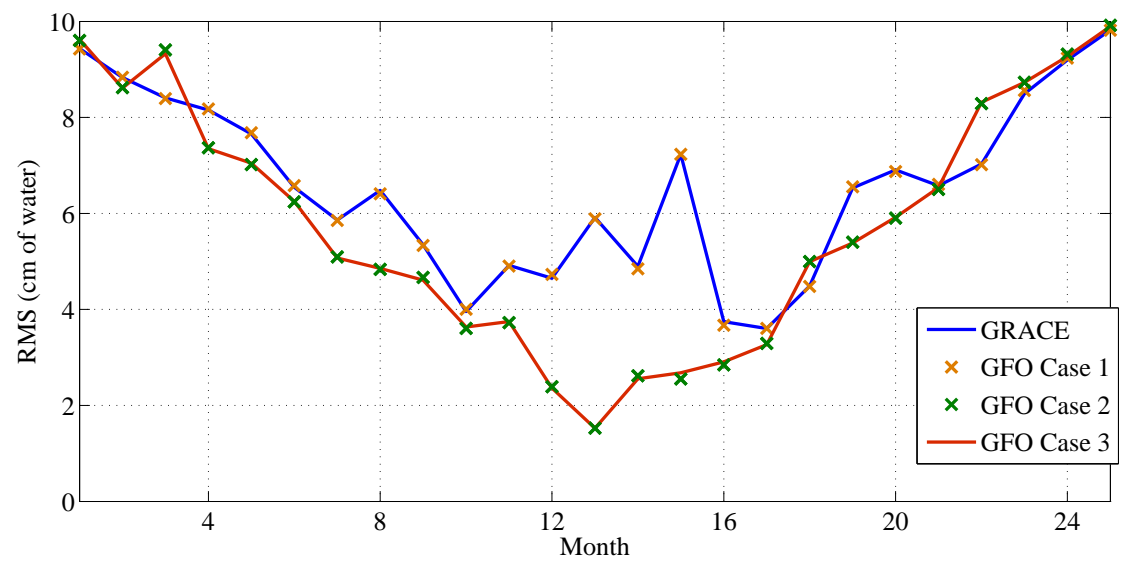

Figure 5.47: RMS errors of Greenland spatial maps for all mission configurations: all error sources.

\begin{tabular}{|l|l|}
\hline $\begin{array}{l}\text { Mission } \\
\text { Configuration }\end{array}$ & $\begin{array}{l}\overline{\mathbf{R M S}} \\
\text { (cm of water) }\end{array}$ \\
\hline GRACE & 6.59 \\
\hline GFO Case 1 & 6.59 \\
\hline GFO Case 2 & 5.78 \\
\hline GFO Case 3 & 5.78 \\
\hline
\end{tabular}

Table 5.13: Mean of monthly RMS of 25 Greenland spatial plots for all errors case. 
The spectral density of the spatial plots for the Truth and the GRACE and GFO Case 3 errors are given in Figure 5.48. This shows moderate improvements for GFO Case 3 at the largest spatial frequencies only.

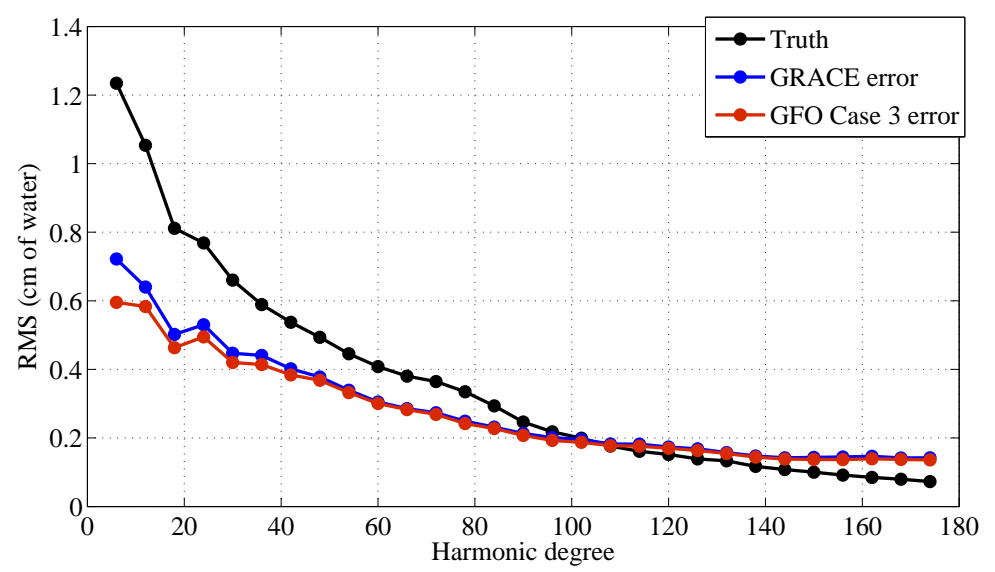

Figure 5.48: Mean spectral density of monthly spatial maps for the Truth signal and GRACE and GFO Case 3 error over Greenland: all error sources.

The time series of mass variations for the Greenland basins and Greenland total are plotted in Figures 5.49 and 5.50 respectively. The error RMS values for each basin and all of Greenland are given in Table 5.14. The GRACE mass variations are noticeably more noisy than the GFO Case 3 solutions, especially for the inland basins (7-12). The larger errors in the GRACE mass estimates is due to the combined effect of the accelerometer errors, and the larger aliasing errors which are present for the higher altitude orbit case.

The estimated values for the trend, annual amplitude, and phase for GRACE and GFO Case 3 are given in Table 5.15 and the errors in the trend and annual amplitude are plotted in Figure 5.51. Even though there are improvements for GFO Case 3 in terms of the error RMS values for the mass variations, it does not result in improved estimates of the trend and amplitude values. 

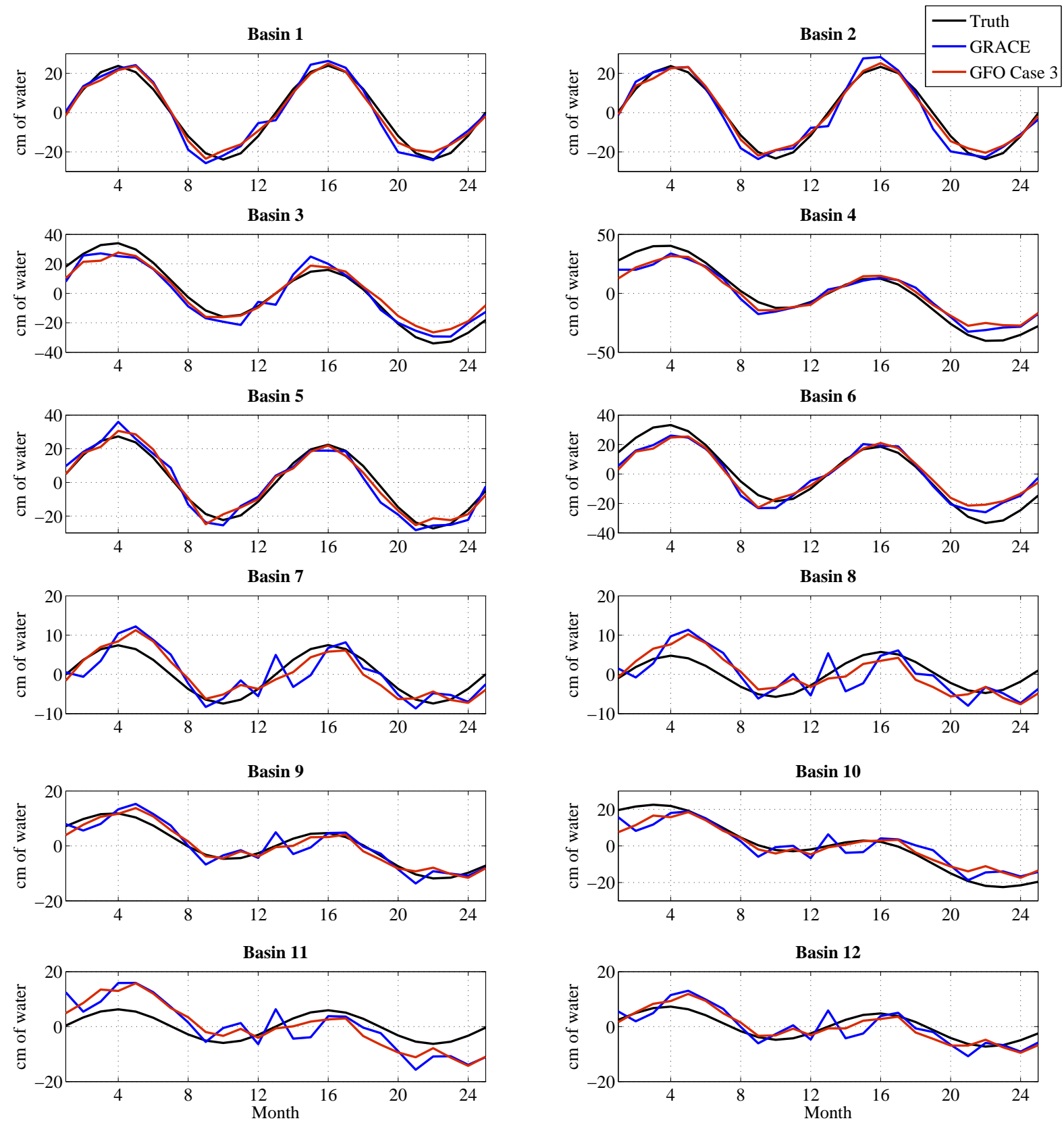

Figure 5.49: Greenland basin mass variations time series for different mission configurations: all error sources. 


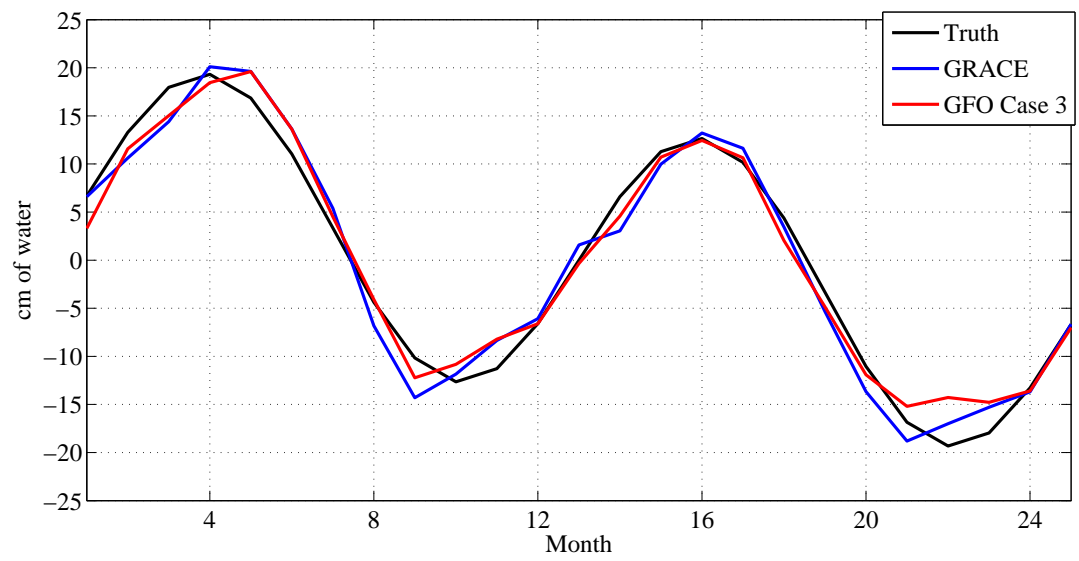

Figure 5.50: Total Greenland variation time series for different mission configurations: all error sources.

\begin{tabular}{|l||l||l|l|}
\hline Basin & $\begin{array}{l}\text { Truth } \\
\text { signal RMS } \\
\text { (cm of water) }\end{array}$ & $\begin{array}{l}\text { GRACE } \\
\text { error RMS } \\
\text { (cm of water) }\end{array}$ & $\begin{array}{l}\text { GFO Case 3 } \\
\text { error RMS } \\
\text { (cm of water) }\end{array}$ \\
\hline 1 & 16.513 & 3.734 & 2.707 \\
\hline 2 & 16.306 & 4.010 & 2.309 \\
\hline 3 & 20.708 & 5.464 & 5.462 \\
\hline 4 & 24.937 & 7.196 & 7.814 \\
\hline 5 & 17.647 & 4.447 & 3.269 \\
\hline 6 & 20.325 & 6.663 & 7.192 \\
\hline 7 & 5.145 & 3.547 & 2.619 \\
\hline 8 & 3.628 & 4.125 & 3.391 \\
\hline 9 & 7.241 & 2.979 & 1.958 \\
\hline 10 & 14.295 & 5.688 & 5.062 \\
\hline 11 & 4.258 & 6.969 & 6.042 \\
\hline 12 & 4.496 & 3.865 & 2.837 \\
\hline Total & 11.959 & 2.183 & 2.081 \\
\hline
\end{tabular}

Table 5.14: RMS of errors for Greenland basin mass estimates for different mission configurations shown in Figure 5.49: all error sources. 


\begin{tabular}{|c|c|c|c|c|c|c|c|c|c|}
\hline \multirow{2}{*}{ Basin } & \multicolumn{3}{|c|}{ Truth } & \multicolumn{3}{|c|}{ GRACE } & \multicolumn{3}{|c|}{ GFO Case 3} \\
\hline & $\begin{array}{l}\text { Trend } \\
(\mathrm{cm})\end{array}$ & $\begin{array}{l}\text { Amp } \\
(\mathrm{cm} / \mathrm{yr})\end{array}$ & $\begin{array}{l}\text { Phase } \\
\text { (deg) }\end{array}$ & $\begin{array}{l}\text { Trend } \\
(\mathrm{cm})\end{array}$ & $\begin{array}{l}\text { Amp } \\
(\mathrm{cm} / \mathrm{yr})\end{array}$ & $\begin{array}{l}\text { Phase } \\
\text { (deg) }\end{array}$ & $\begin{array}{l}\text { Trend } \\
(\mathrm{cm})\end{array}$ & $\begin{array}{l}\text { Amp } \\
(\mathrm{cm} / \mathrm{yr})\end{array}$ & $\begin{array}{l}\text { Phase } \\
\text { (deg) }\end{array}$ \\
\hline 1 & 0.13 & 23.88 & -0.00 & 0.06 & 24.61 & 4.42 & -0.18 & 22.38 & 1.19 \\
\hline 2 & -0.39 & 23.41 & -0.00 & -0.52 & 24.60 & 4.70 & -0.56 & 22.34 & 1.25 \\
\hline 3 & -18.07 & 20.44 & -0.00 & -10.50 & 22.45 & 3.20 & -9.47 & 20.08 & 0.15 \\
\hline 4 & -27.89 & 19.35 & -0.00 & -16.99 & 19.39 & -1.59 & -15.79 & 18.64 & -2.72 \\
\hline 5 & -4.96 & 23.61 & -0.00 & -8.51 & 23.76 & 5.30 & -6.94 & 22.34 & 1.06 \\
\hline 6 & -14.70 & 22.24 & -0.00 & -5.04 & 22.50 & 4.94 & -4.35 & 20.76 & 1.06 \\
\hline 7 & 0.04 & 7.44 & -0.00 & -1.99 & 6.49 & -15.48 & -2.76 & 6.14 & -11.20 \\
\hline 8 & 0.97 & 5.48 & -0.00 & -3.10 & 4.72 & -23.32 & -3.80 & 4.41 & -17.66 \\
\hline 9 & -7.16 & 6.47 & -0.00 & -7.84 & 6.11 & -10.95 & -7.57 & 5.76 & -12.04 \\
\hline 10 & -19.58 & 7.12 & -0.00 & -13.06 & 6.43 & -16.14 & -12.46 & 6.54 & -13.98 \\
\hline 11 & -0.36 & 6.03 & -0.00 & -10.51 & 5.24 & -16.44 & -9.95 & 5.35 & -15.61 \\
\hline 12 & -2.48 & 5.40 & -0.00 & -5.82 & 4.68 & -16.86 & -5.91 & 4.47 & -16.31 \\
\hline
\end{tabular}

Table 5.15: Best fit trend, annual amplitude, and phase values for Greenland basins: all error sources.
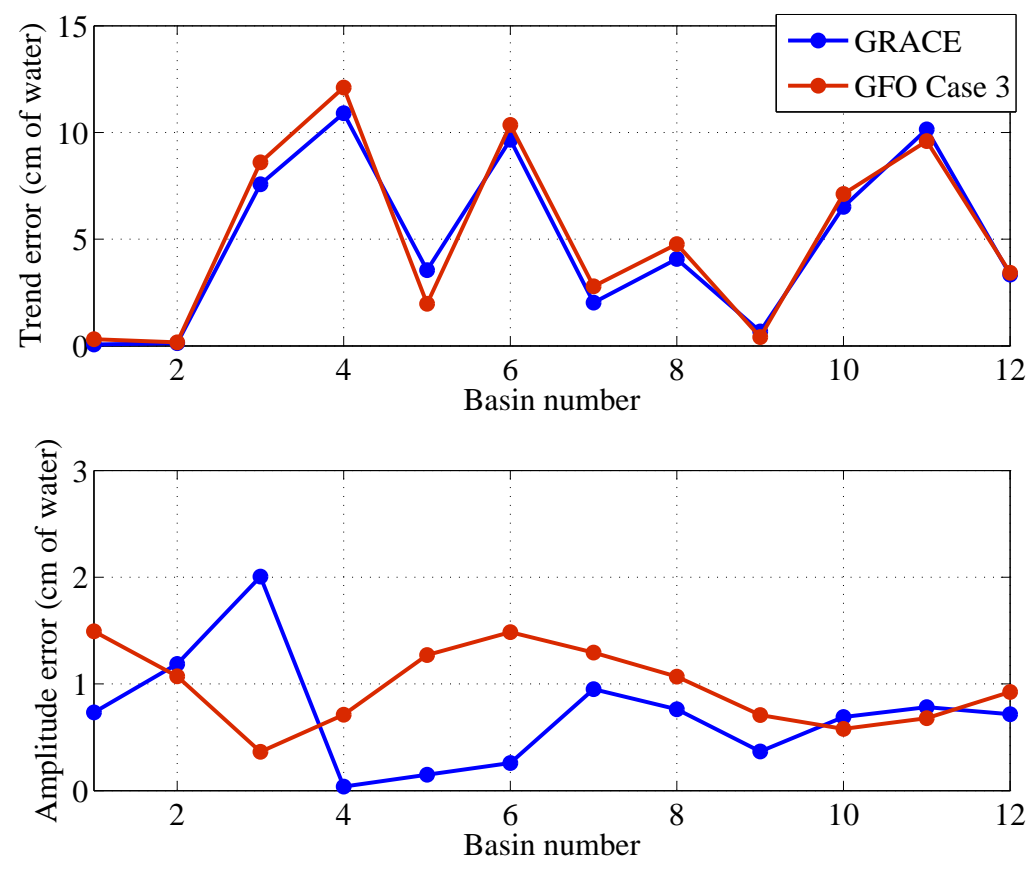

Figure 5.51: Errors in trend and amplitude estimates for each Greenland basin for GRACE and GFO Case 3: all error sources. 


\subsection{Effects of Temporal Aliasing}

This section explores the effect that temporal aliasing has on the errors of the simulations. As the results in this section show, temporal aliasing is the dominant error source on the simulated gravity estimates, and the reason that there are not dramatic differences for the results of the different mission configurations presented in Section 5.5 .

\subsubsection{Satellite Altitude Sensitivity to Aliasing}

The results presented here, show the effect that the satellite altitude has on the temporal aliasing errors for regional mascon estimates. For these simulations, the Truth signal is defined by the ECMWF/OMCT AOD signal and the FES2004 tidal signal, and the Nominal signal is defined by the NCEP/MOG2D AOD signal and the GOT00 tidal signal. The estimated signal is thus the approximate effect that these errors have on the solutions previously presented. The simulations are performed for the two different orbits considered in this study, where one has an altitude of $\sim 480 \mathrm{~km}$ and the other has an altitude of $\sim 250 \mathrm{~km}$.

The recovered temporal aliasing error over South America for the two orbits are shown in Figures 5.52 and 5.53. The aliasing errors are clearly larger for the $480 \mathrm{~km}$ orbit than for the $250 \mathrm{~km}$ orbit. This difference in aliasing errors is caused by the difference in the satellite altitude and the difference in the spatial sampling of the two orbits as shown in Figure 3.9.

The corresponding time series of mass variations due to the temporal aliasing error for the two orbits is shown in Figure 5.54. The Truth signal is included on the plots as a point of comparison. Table 5.16 gives the RMS values of the errors showing 
that the higher altitude orbit consistently has higher aliasing errors, though significant differences are only observed for basins 7 and 8 . 

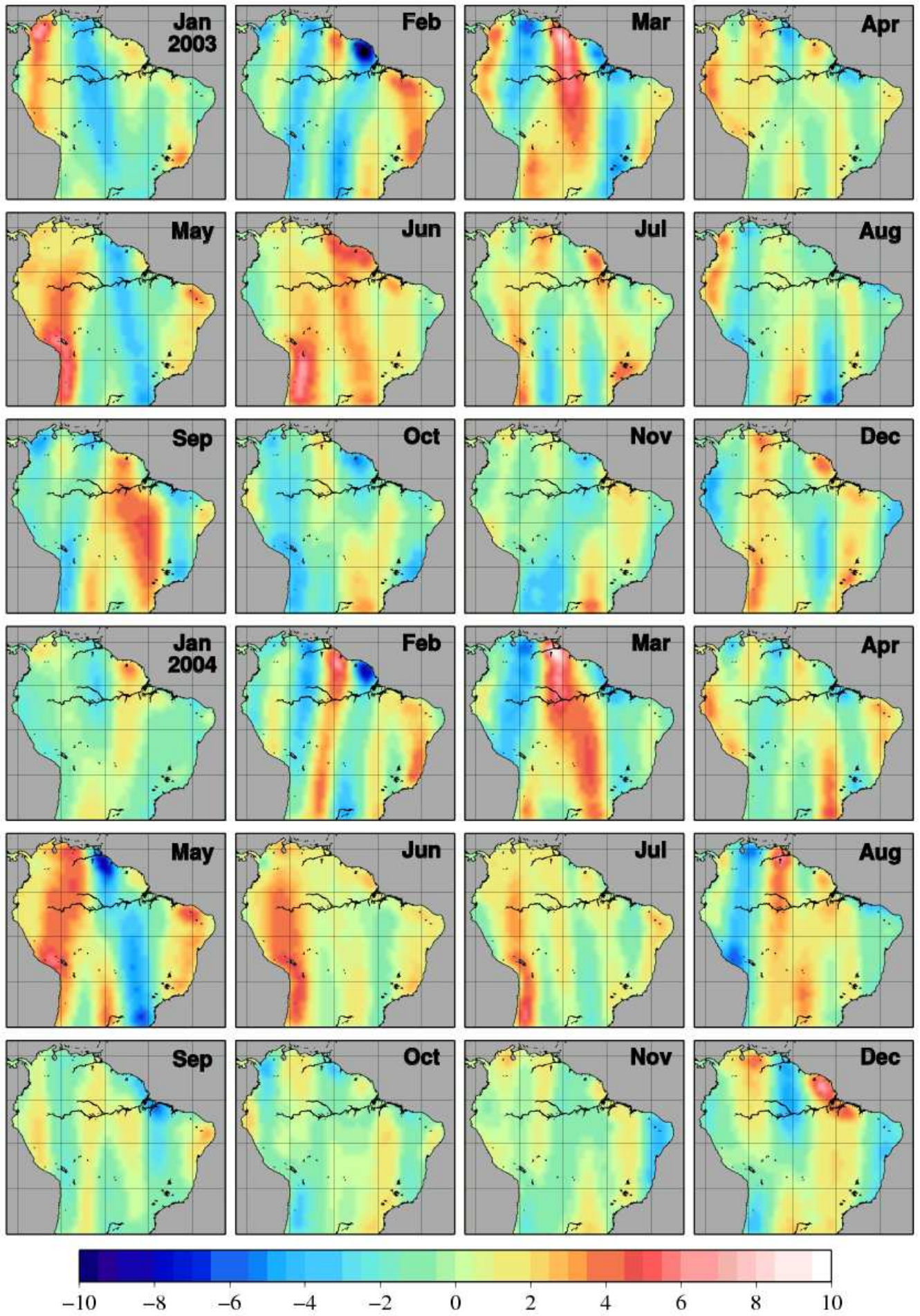

cm of water

Figure 5.52: South America temporal aliasing error for the $480 \mathrm{~km}$ orbit. 

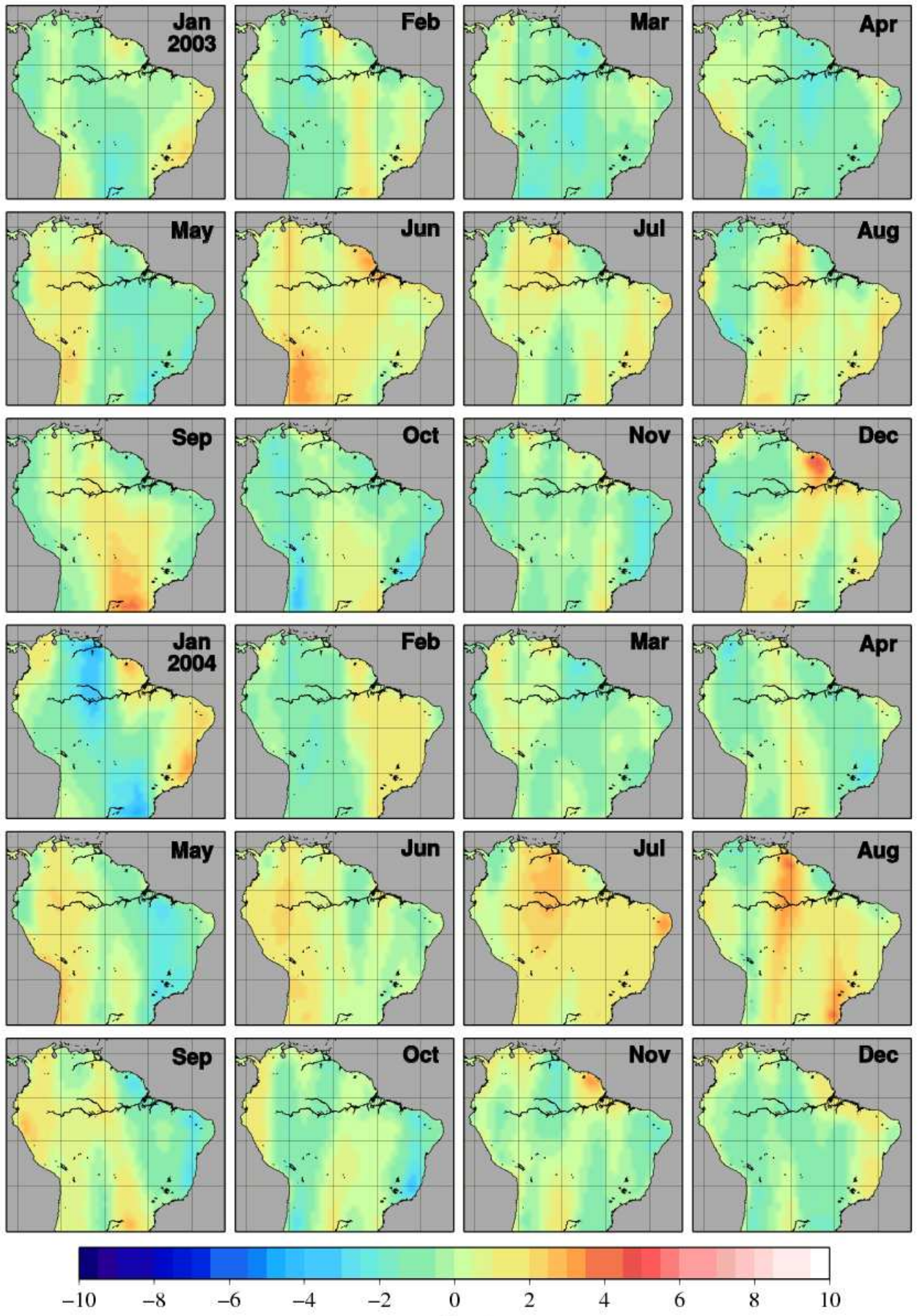

cm of water

Figure 5.53: South America temporal aliasing error for the $250 \mathrm{~km}$ orbit. 

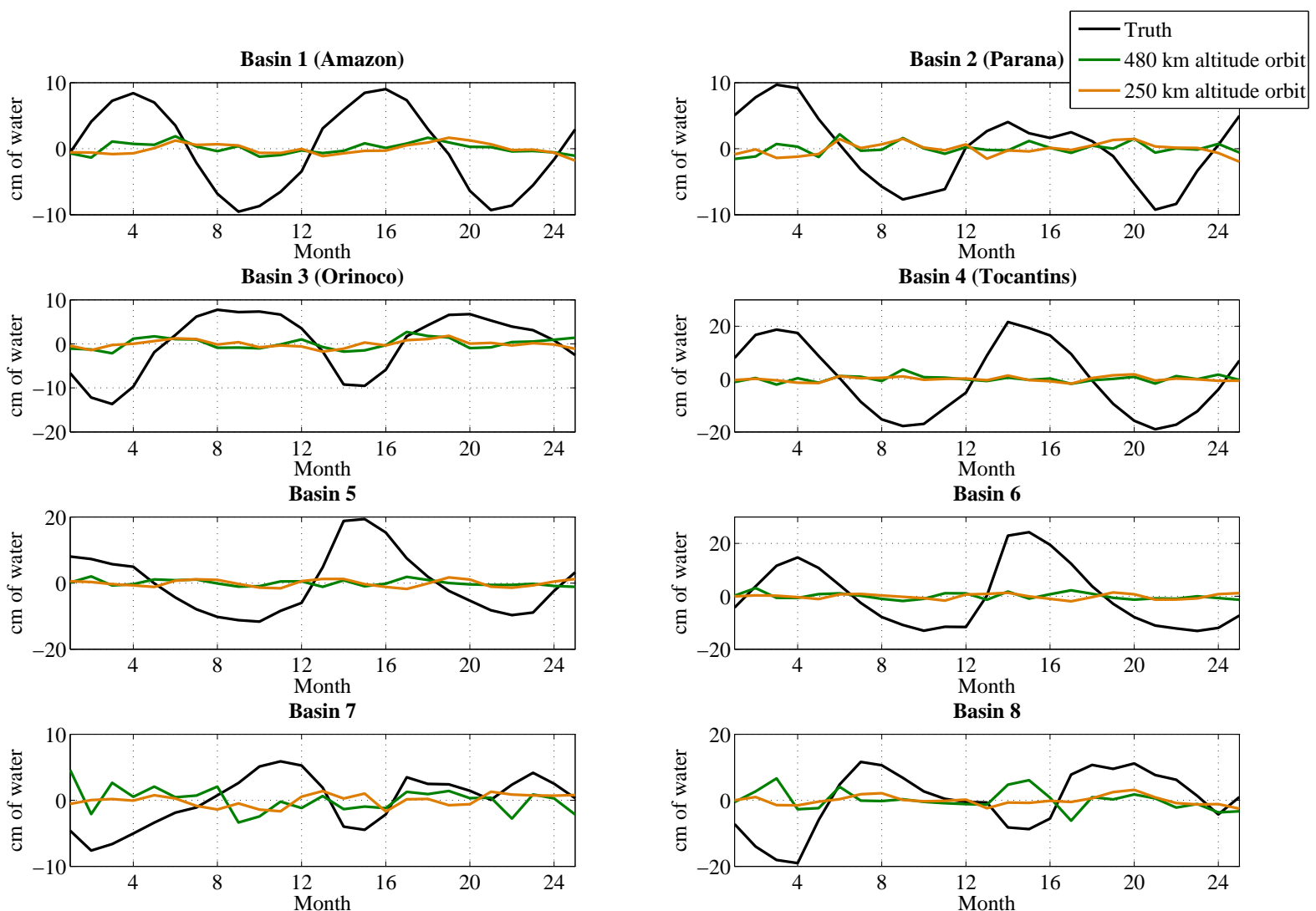

Figure 5.54: Mass variations for South America basins from temporal aliasing.

\begin{tabular}{|l|l|l|}
\hline Basin & $\begin{array}{l}\text { 480 km altitude } \\
\text { signal RMS } \\
\text { (cm of water) }\end{array}$ & $\begin{array}{l}\text { 250 } \mathbf{k m} \text { altitude } \\
\text { signal RMS } \\
\text { (cm of water) }\end{array}$ \\
\hline 1 & 0.853 & 0.816 \\
\hline 2 & 0.892 & 0.923 \\
\hline 3 & 1.278 & 0.847 \\
\hline 4 & 1.214 & 0.890 \\
\hline 5 & 0.910 & 1.040 \\
\hline 6 & 1.233 & 0.939 \\
\hline 7 & 1.823 & 0.894 \\
\hline 8 & 2.956 & 1.391 \\
\hline
\end{tabular}

Table 5.16: RMS of temporal aliasing signal over South America. 
The spatial maps of the temporal aliasing error over Greenland for the two different orbits are shown in Figures 5.55 and 5.56. Once again, the aliasing errors are noticeably larger for the higher altitude orbit and is caused by the difference in altitude and the way the groundtracks sample the time-variable signal.

The mass variations for the Greenland basins due to aliasing are plotted in Figure 5.57 and Table 5.17 gives the RMS of the error for the two orbits. The difference in the errors between the two orbit scenarios is much larger for the Greenland aliasing error than for South America. GFO Case 3 has lower errors for all twelve Greenland basins. 

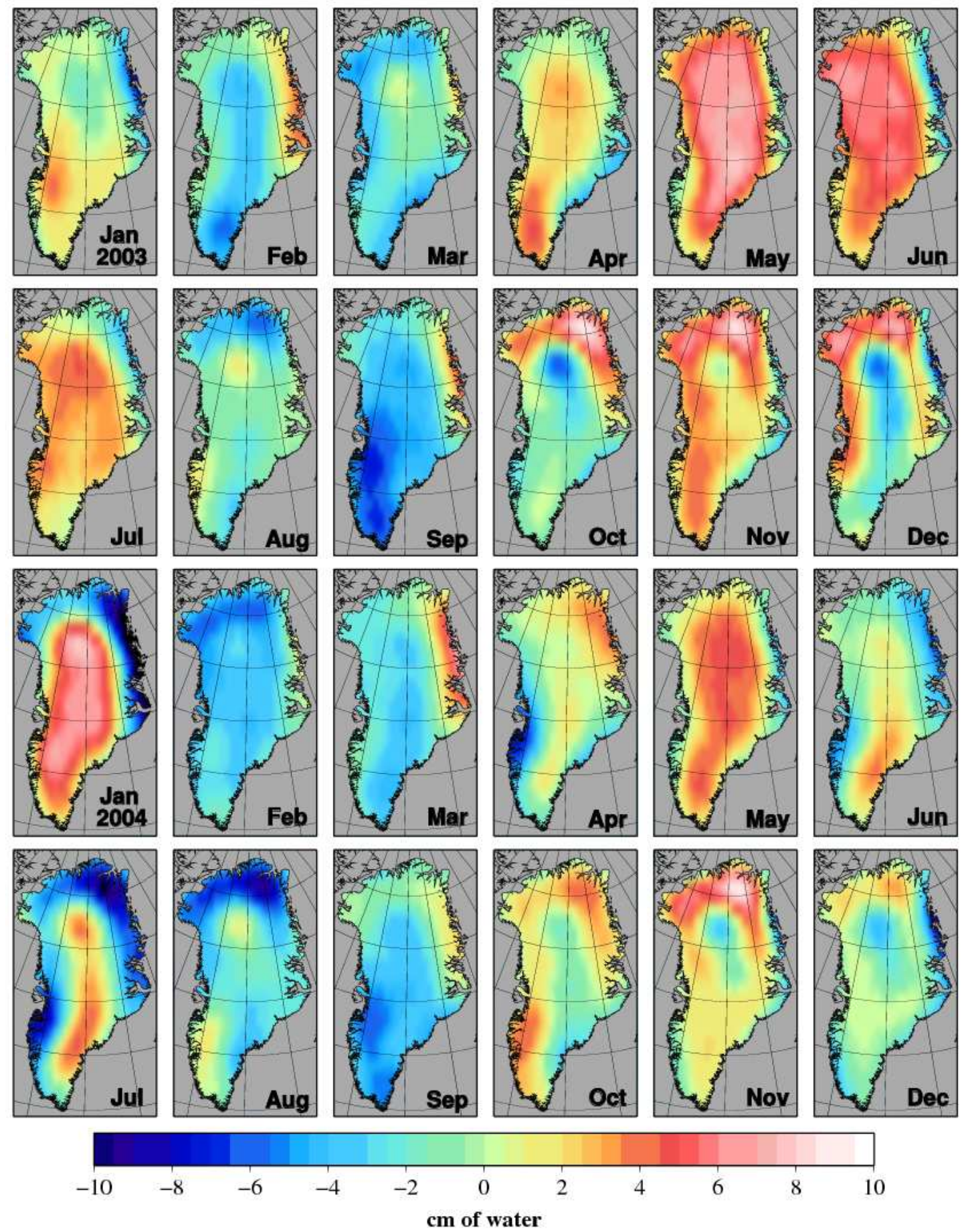

Figure 5.55: Greenland temporal aliasing error for the $480 \mathrm{~km}$ orbit. 

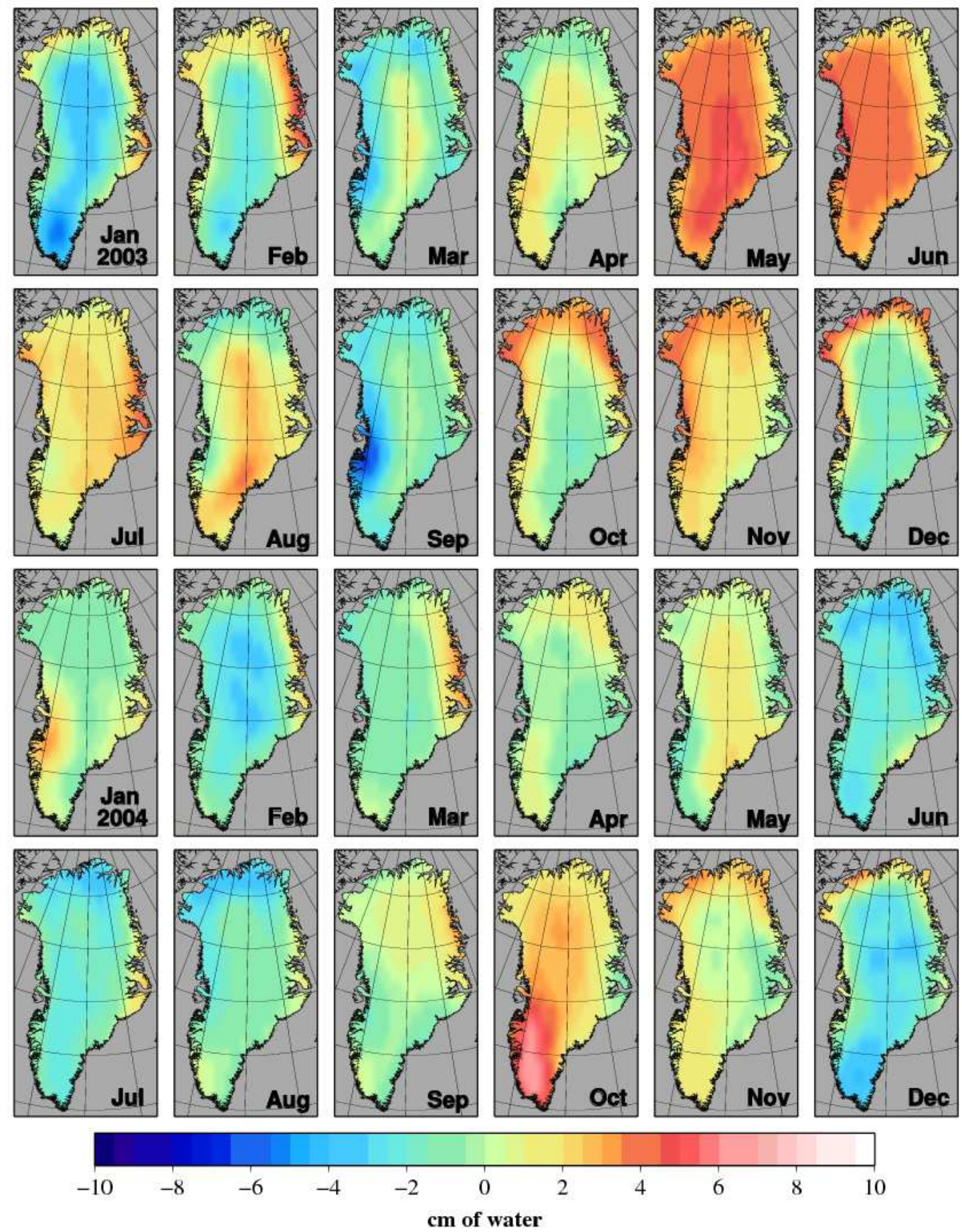

Figure 5.56: Greenland temporal aliasing error for the $250 \mathrm{~km}$ orbit. 

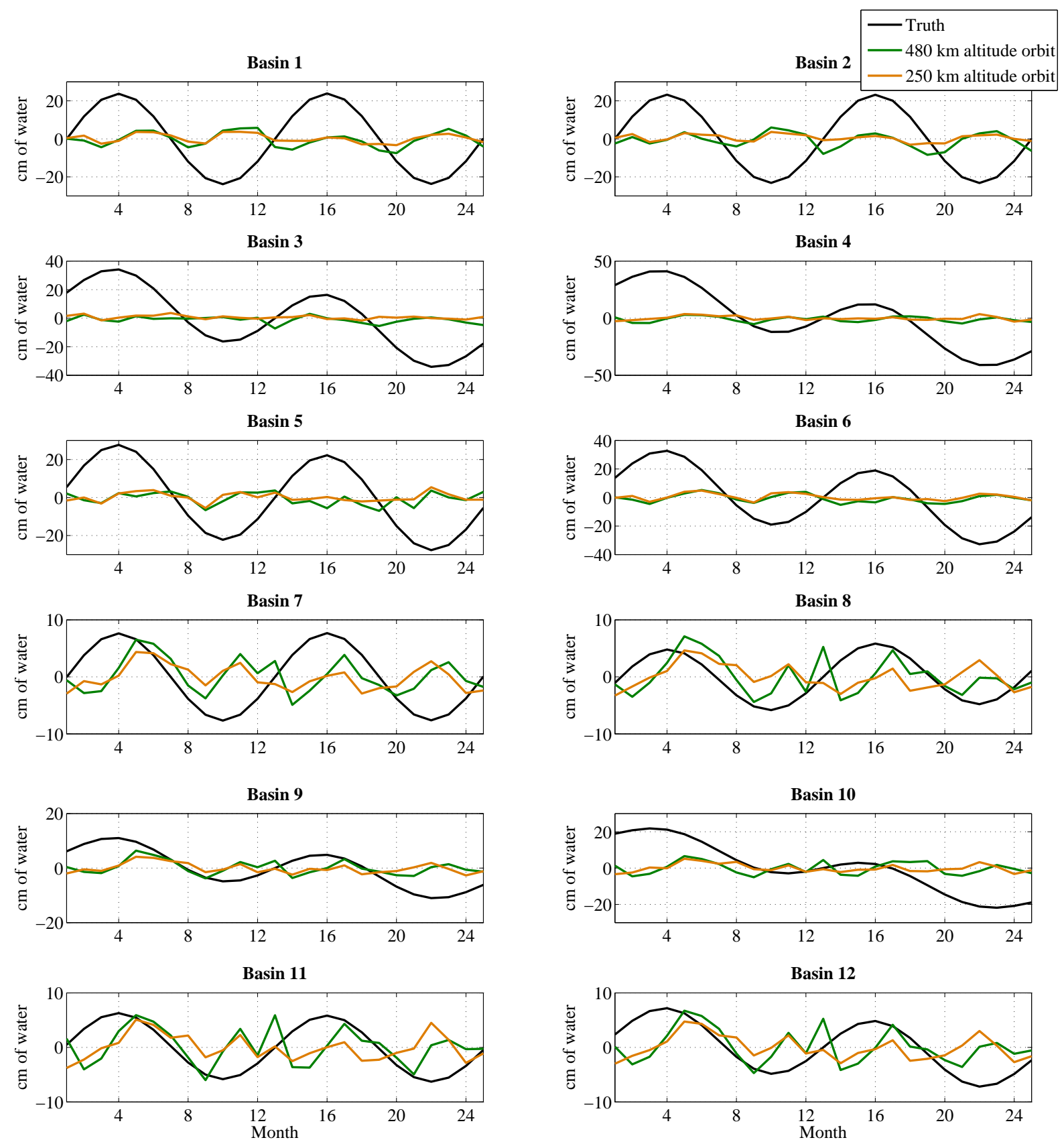

Figure 5.57: Mass variations for Greenland basins from temporal aliasing. 


\begin{tabular}{|l|l|l|}
\hline Basin & $\begin{array}{l}\text { 480 km altitude } \\
\text { signal RMS } \\
\text { (cm of water) }\end{array}$ & $\begin{array}{l}\text { 250 km altitude } \\
\text { signal RMS } \\
\text { (cm of water) }\end{array}$ \\
\hline 1 & 4.730 & 2.360 \\
\hline 2 & 4.756 & 1.987 \\
\hline 3 & 3.023 & 1.607 \\
\hline 4 & 2.986 & 2.191 \\
\hline 5 & 4.172 & 2.808 \\
\hline 6 & 3.777 & 2.547 \\
\hline 7 & 3.112 & 2.098 \\
\hline 8 & 3.172 & 2.133 \\
\hline 9 & 2.854 & 1.971 \\
\hline 10 & 3.433 & 2.343 \\
\hline 11 & 3.318 & 2.323 \\
\hline 12 & 3.128 & 2.150 \\
\hline
\end{tabular}

Table 5.17: RMS of temporal aliasing signal over Greenland.

\subsubsection{Separation of AOD and Tidal Aliasing Effects}

This results presented here reveal the relative contributions to the total aliasing error caused by imperfections in the models by separately simulating the effects of the AOD errors and the tidal errors. To simulate the AOD errors only, the Truth signal consists of the ECMWF/OMCT AOD signal, and the Nominal signal consists of the NCEP/MOG2D signal. To simulate the tidal errors only, the Truth signal is the FES2004 tide model, and the Nominal signal is the GOT00 tide model.

The plots showing the monthly error RMS values of the different components for South America and Greenland are shown in Figures 5.58 and 5.59 respectively. The results show that in general the AOD errors tend to dominate, but that the tidal errors are also significant. Both the AOD and tidal errors are largest for the Greenland 480 $\mathrm{km}$ altitude simulations. 


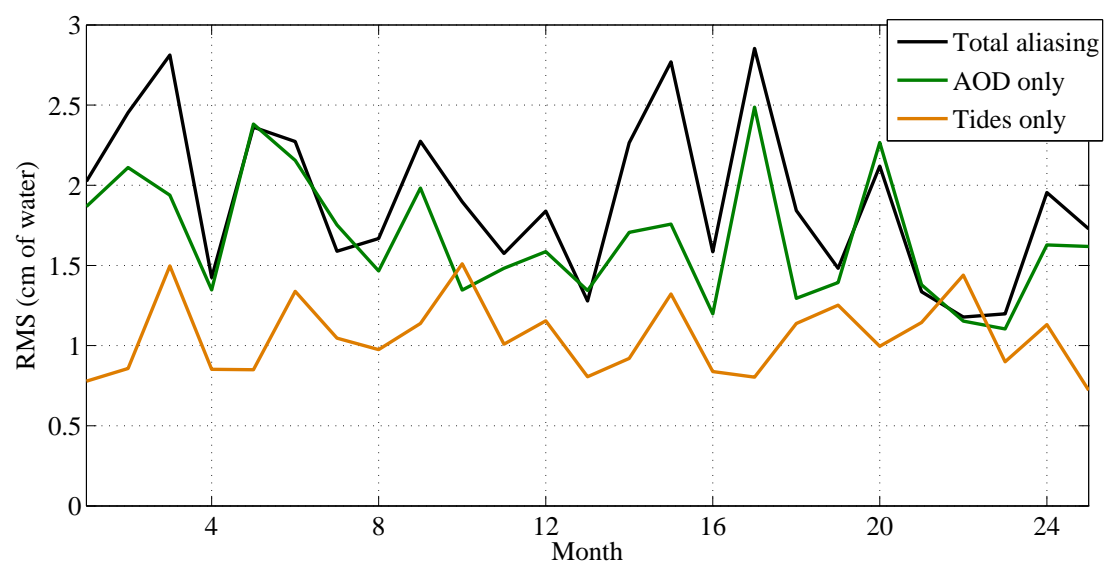

(a) $480 \mathrm{~km}$ altitude orbit

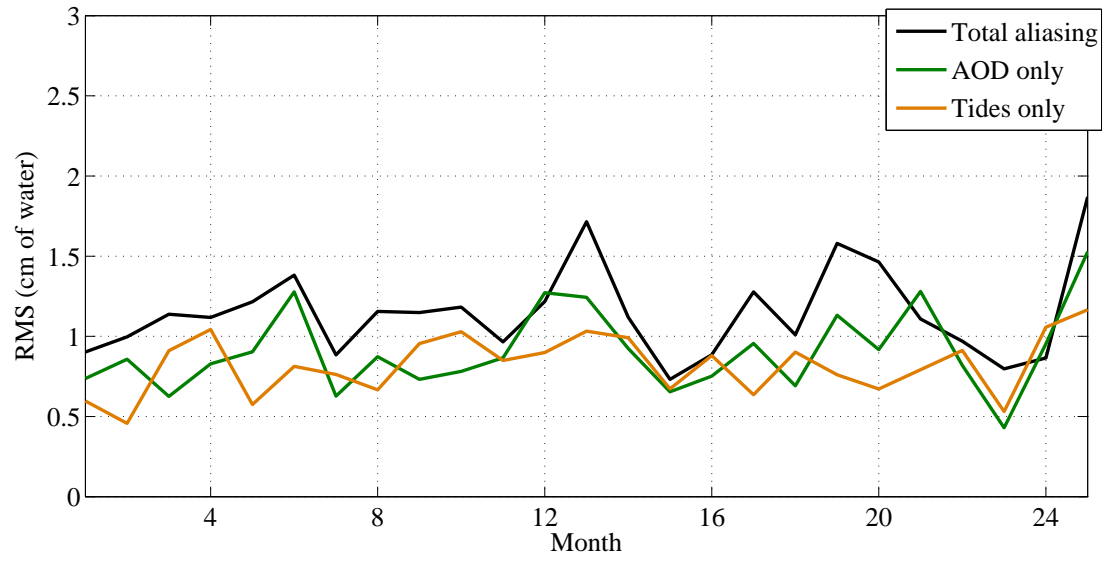

(b) $250 \mathrm{~km}$ altitude orbit

Figure 5.58: Separation of aliasing components for simulations over South America. 


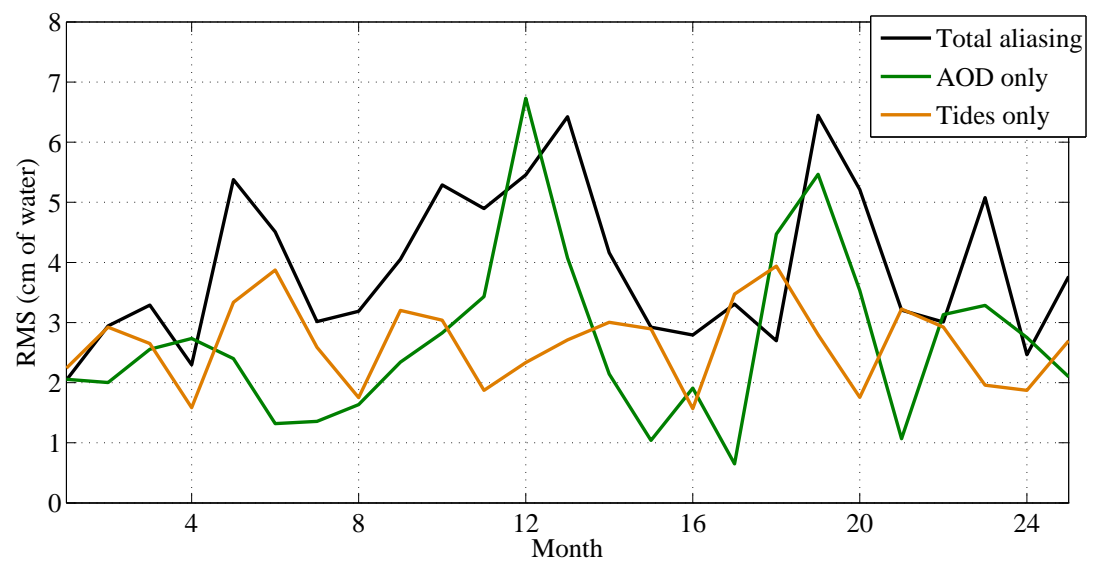

(a) $480 \mathrm{~km}$ altitude orbit

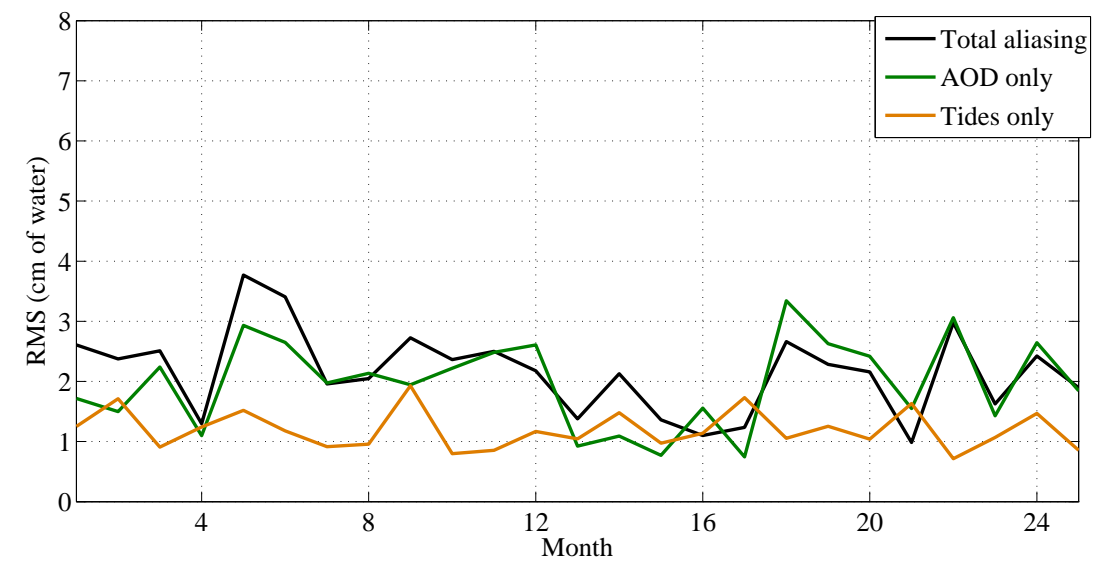

(b) $250 \mathrm{~km}$ altitude orbit

Figure 5.59: Separation of aliasing components for simulations over Greenland. 


\subsubsection{Measuring the Effect of Hydrological Aliasing}

In addition to the temporal aliasing errors introduced by the imperfections in the AOD and tidal models, aliasing errors are also present due to the high temporal frequency in the hydrological signal. The effect of the hydrological aliasing on both orbits is determined by simulating cases where the only signal is the GLDAS hydrological signal, where no instrument noise has been added to the simulations.

The plotted data in Figure 5.60 shows the monthly spatial error RMS values for the hydrological aliasing, along with plots for the total error, the total effect of the aliasing error, and the AOD and tidal aliasing. The solid black line in the two plots in Figure 5.60 is the total error for the GRACE and GFO Case 3 missions respectively. The red marks show the errors for the case where no instrument noise is included in the simulations, and only the aliasing errors are present. This shows that for GFO Case 3, the aliasing errors only case, nearly exactly matches the case where all error sources are included in the simulation, showing that the mission errors are entirely determined by the aliasing error. Even for GRACE, the level of accuracy for the aliasing errors only case is very close to the case where all errors are included, proving that the aliasing errors dominate. The results also show that for South America, where the AOD errors are relatively low, that the aliasing caused by the hydrological signal is dominant over the AOD and tidal aliasing errors. Also, the level of hydrological errors is similar for the two orbits, as opposed to the AOD and tidal aliasing where a noticeable improvement is seen for the lower altitude orbit. 


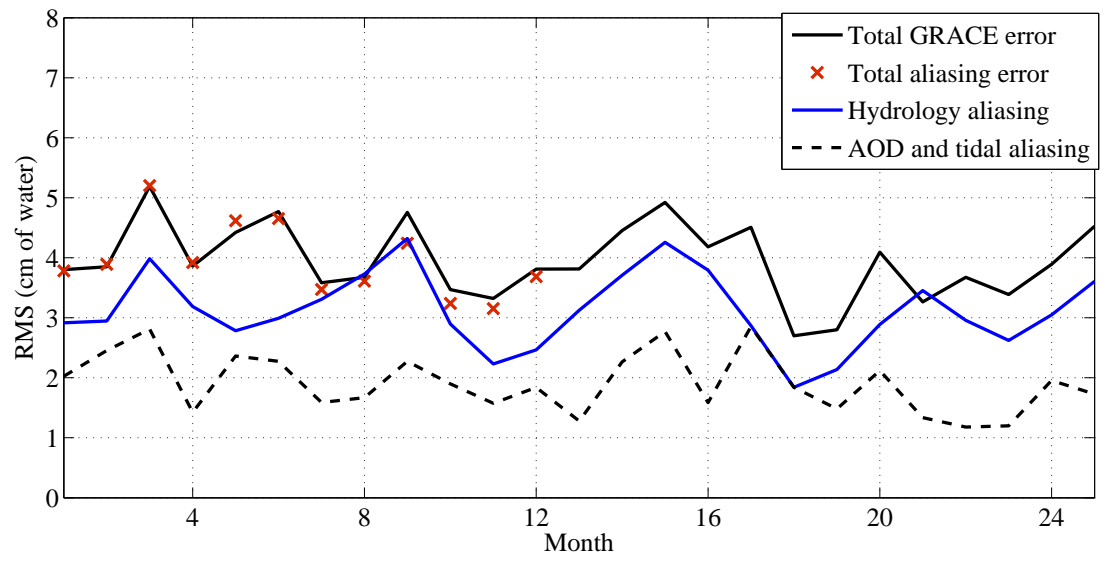

(a) $480 \mathrm{~km}$ altitude orbit

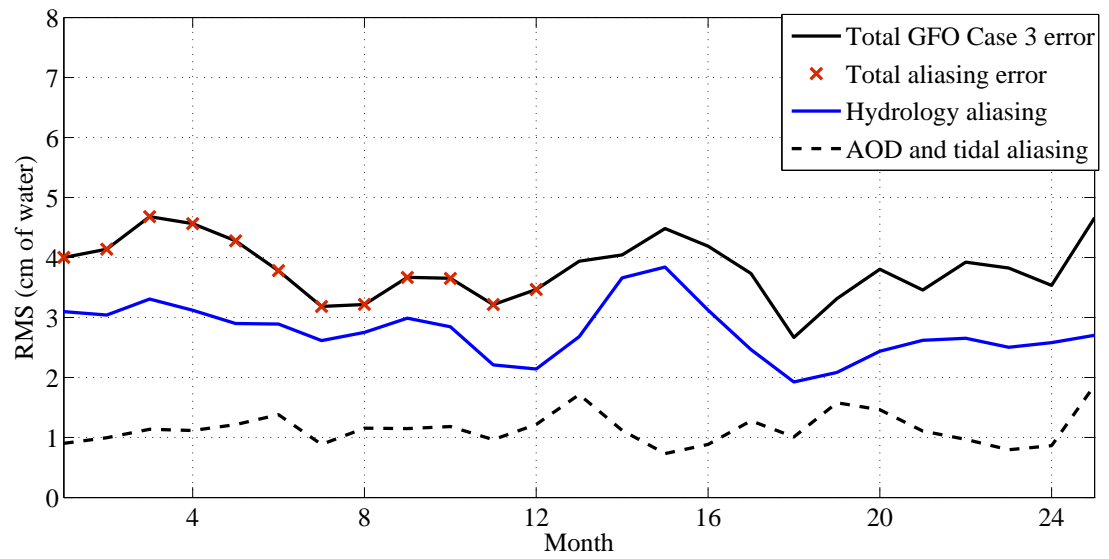

(b) $250 \mathrm{~km}$ altitude orbit

Figure 5.60: Total errors and aliasing error components for South America simulations. 


\subsection{Mass Variation Estimates From Mascons and Averaging Ker- nels}

The results presented in this section explore the difference in basin mass variations as determined with the mascon approach and the application of averaging kernels to monthly sets of spherical harmonics. The spherical harmonic solutions are formed using the same set of partial derivatives which are used to form the mascon solutions. The averaging kernel technique discussed in Section 2.5 is then applied to the resulting monthly sets of spherical harmonics and plotted as a time series of mass variations for each basin. The parameters which define the averaging kernel for each basin are varied such that the error RMS is minimized. A scale factor is calculated for each considered averaging kernel. The GRACE mission is the simulated case considered in this section.

Figure 5.61 shows the mass variation time series for the South America basins as determined with mascons and the averaging kernel method. The error RMS values for the two techniques for all basins are given in Table 5.18. The results show that the averaging kernel method performs as well or better than the mascon estimation method for the largest basins (1-3). As the size of the basins decreases the mascon method performs significantly better than the averaging kernels. This improvement is due to the spatial constraints which are applied with the mascon approach. The noisy estimates which result from the averaging kernel are due to leakage of the signal from outside the basin. The leakage error also causes the calculated scale factor to be large resulting in an estimated signal that is too large.

The mass variation time series for the Greenland basins and the total Greenland signal are shown in Figures 5.62 and 5.63 respectively. There is very little difference in performance between the two techniques at estimating the mass changes over all of 
Greenland, with the averaging kernel method yielding a more accurate result. There are significant differences, however, between the two methods for the basin mass variation estimates. The averaging kernel estimates are similar in accuracy to the mascon estimates for the coastal basins, where the Truth signal is largest. The mass estimates for the inland basins using the averaging kernel are very noisy as compared to the mascon estimates. Again, this discrepancy between the techniques is due to the fact that the mascon setup allows for the application of spatial constraints which results in a smoothed estimate. The large annual amplitudes of the inland basin estimates show that the large signal from the coastal basins leaks into the inland basins.

The improvement in the mascon estimates observed in this section is due to the additional information introduced into the estimation process by the spatial constraints, as they prevent the estimates at smaller spatial scales from becoming too noisy. If a similar constraining procedure were to be applied to spherical harmonics, it is expected that the results would be similar in accuracy to those obtained by estimating constrained mascons. The results in this section simply show that the standard averaging kernel technique should not be applied to smaller basins because the leakage errors are large. 

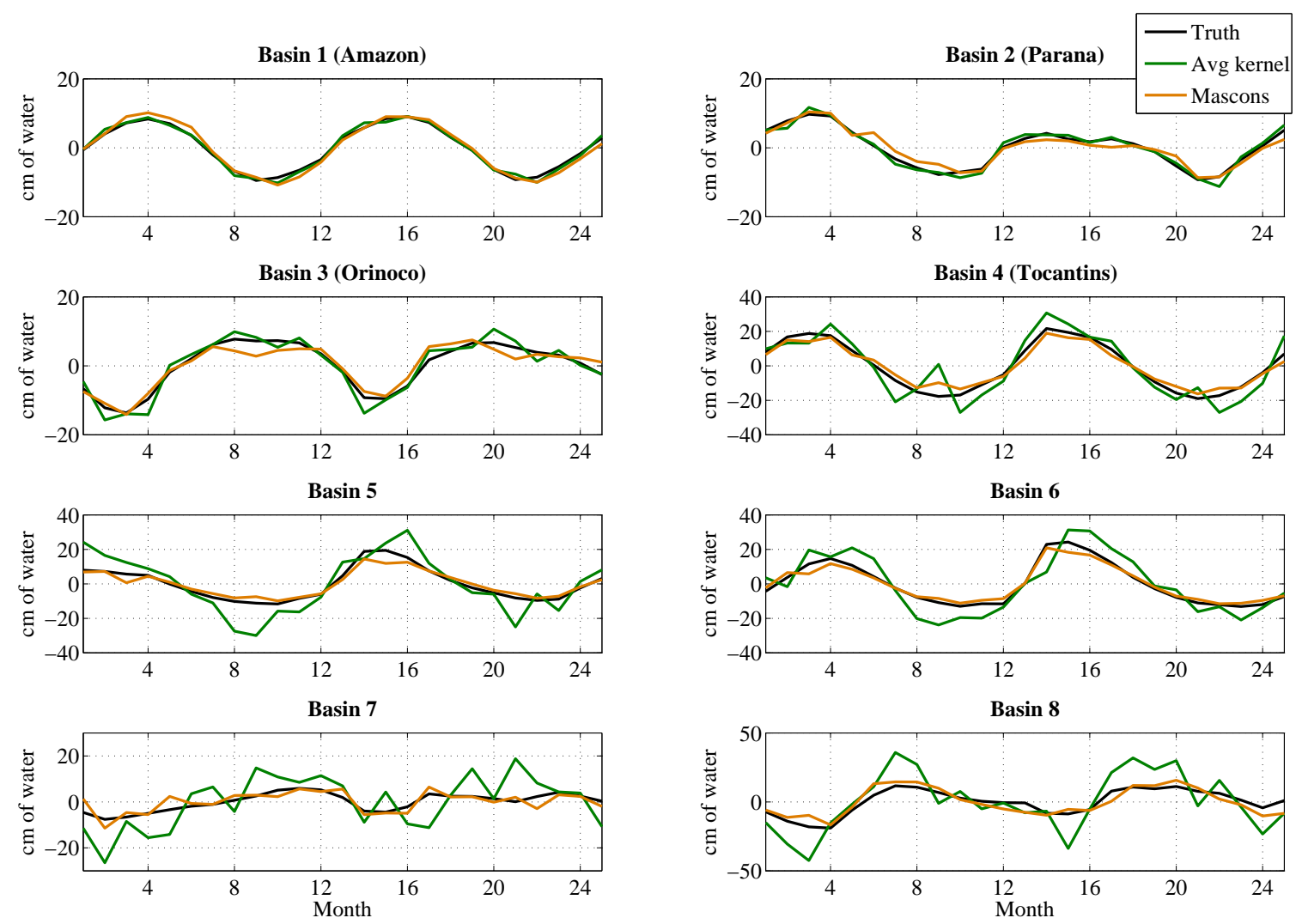

Figure 5.61: South America basin mass variations for averaging kernel and mascon estimation methods.

\begin{tabular}{|l||l||l|l|}
\hline Basin & $\begin{array}{l}\text { Truth } \\
\text { signal RMS } \\
\text { (cm of water) }\end{array}$ & $\begin{array}{l}\text { Averaging } \\
\text { kernel } \\
\text { error RMS } \\
\text { (cm of water })\end{array}$ & $\begin{array}{l}\text { Mascons } \\
\text { error RMS } \\
\text { (cm of water })\end{array}$ \\
\hline 1 & 6.222 & 0.829 & 1.243 \\
\hline 2 & 5.459 & 1.151 & 1.617 \\
\hline 3 & 6.705 & 2.110 & 2.106 \\
\hline 4 & 13.609 & 7.215 & 3.177 \\
\hline 5 & 9.076 & 8.787 & 2.587 \\
\hline 6 & 11.844 & 7.760 & 2.438 \\
\hline 7 & 3.790 & 8.993 & 2.642 \\
\hline 8 & 8.907 & 13.422 & 4.546 \\
\hline
\end{tabular}

Table 5.18: RMS of errors for South America basin mass estimates for averaging kernel and mascons: GRACE mission with all errors included. 

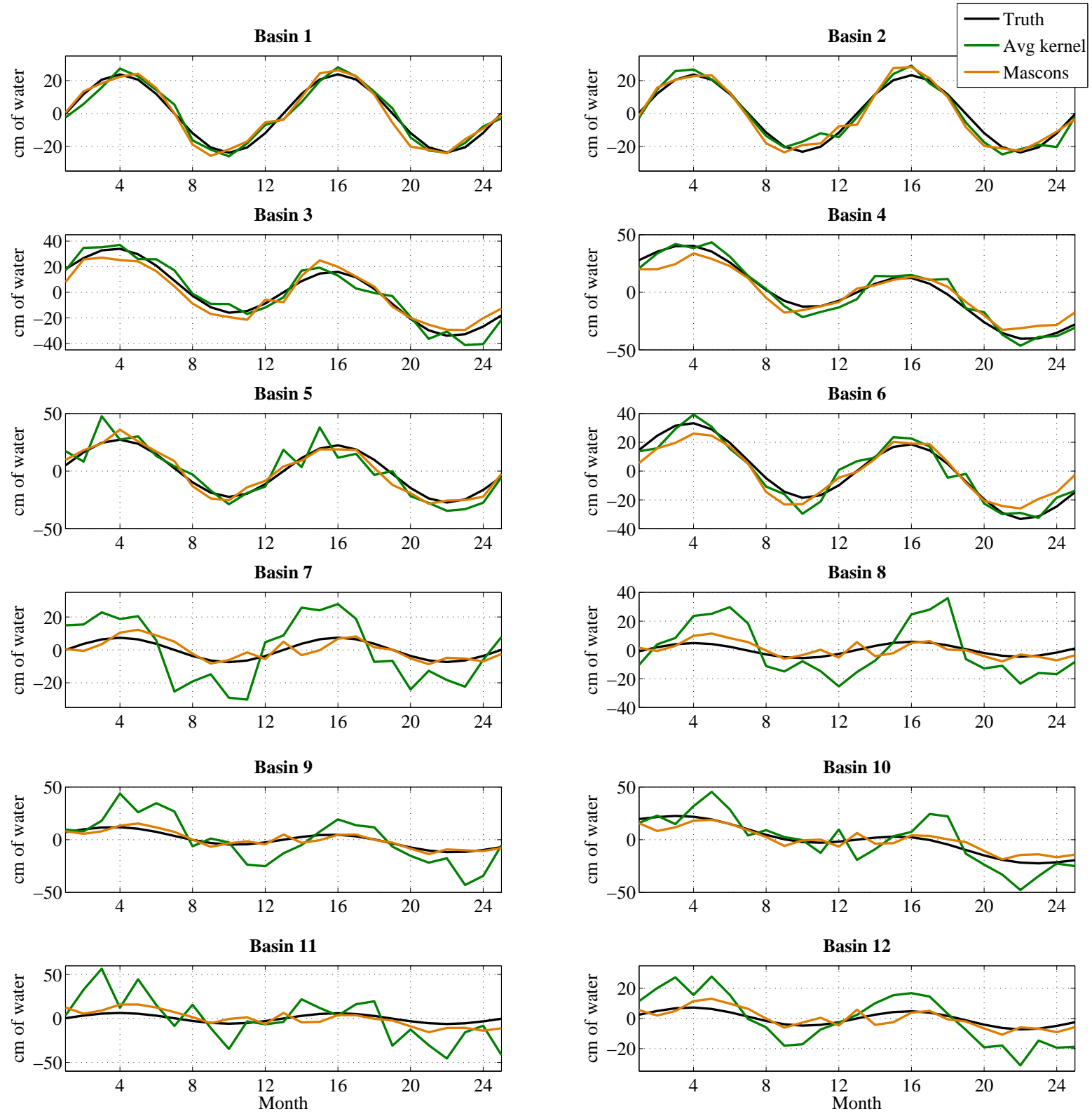

Figure 5.62: Greenland basin mass variations for averaging kernel and mascon estimation methods. 


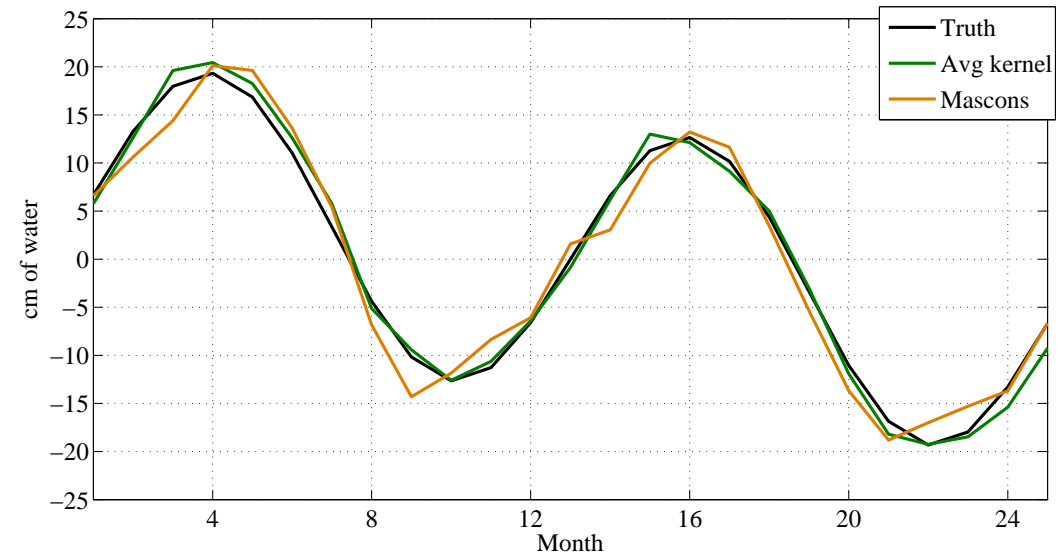

Figure 5.63: Total Greenland mass variations for averaging kernel and mascon estimation methods.

\begin{tabular}{|l||l||l|l|}
\hline Basin & $\begin{array}{l}\text { Truth } \\
\text { signal RMS } \\
\text { (cm of water) }\end{array}$ & $\begin{array}{l}\text { Averaging } \\
\text { kernel } \\
\text { error RMS } \\
\text { (cm of water) }\end{array}$ & $\begin{array}{l}\text { Mascons } \\
\text { error RMS } \\
\text { (cm of water })\end{array}$ \\
\hline 1 & 16.513 & 3.389 & 3.734 \\
\hline 2 & 16.306 & 3.930 & 4.010 \\
\hline 3 & 20.708 & 5.716 & 5.464 \\
\hline 4 & 24.937 & 5.406 & 7.196 \\
\hline 5 & 17.647 & 9.497 & 4.447 \\
\hline 6 & 20.325 & 5.443 & 6.663 \\
\hline 7 & 5.145 & 14.850 & 3.547 \\
\hline 8 & 3.628 & 15.647 & 4.125 \\
\hline 9 & 7.241 & 15.583 & 2.979 \\
\hline 10 & 14.295 & 13.150 & 5.688 \\
\hline 11 & 4.258 & 22.239 & 6.969 \\
\hline 12 & 4.496 & 12.246 & 3.865 \\
\hline Total & 11.959 & 1.210 & 2.183 \\
\hline
\end{tabular}

Table 5.19: RMS of errors for Greenland basin mass estimates for averaging kernel and mascons: GRACE mission with all errors included. 


\section{Chapter 6}

\section{Conclusions}

This chapter summarizes the results presented in the previous chapter and makes recommendations for future gravity satellite missions and further research which would benefit the design of such a mission.

\subsection{Summary and Conclusions}

The first set of mascon results presented in the previous chapter explored a few different constraint scenarios. The results showed that the setup of the constraints can have a large effect on the accuracy of the estimates. The least accurate simulated estimates were for the scenario where only the mascons within the defined basins were constrained to each other. The most accurate estimates were achieved when the setup of the constraints best accounted for the characteristics of the geophysical signals estimated by the simulations. For the South America simulations, the best results were obtained by separately constraining the land and ocean mascons, since the geophysical signals over neighboring land and ocean mascons are unrelated. The ideal Greenland estimates were formed by introducing an additional constraint matrix which took into account the fact that the signal annual amplitude is dependent on the mascon elevation. These

simulations showed that prior knowledge of the geophysical signals should be used in 
constructing mascon constraints to optimally estimate time-variable gravity.

The simulated gravity estimates reveal that the dominant error source for all mission configurations is temporal aliasing. The effect that temporal aliasing has on the regional mascon estimates is determined by the magnitude of the high-frequency variations in the signal, the satellite altitude, and the spatial and temporal sampling of the signal due to the satellite groundtrack. Due to the spatial variability of the errors in the AOD and tidal models, and the variations of the hydrological signal, the accuracy of regional gravity estimates can vary significantly based on location. For example, the results showed that temporal aliasing of the hydrological signal is the dominant error source for gravity estimation over South America, while the aliasing errors from AOD and tidal model errors are dominant over Greenland. Aliasing errors caused by AOD errors were consistently larger than those from tidal error, but the tidal errors were still significant.

Inclusion of the laser interferometer on a GRACE follow-on mission only improves the accuracy of the gravity estimates for cases where high-frequency variations in the signal do not exit. This was shown in the results by the fact that there was no observable difference between GRACE and GFO Case 1 performance, and between GFO Case 2 and GFO Case 3 performance, for South America hydrology estimates, or Greenland estimates where AOD and tidal errors are included.

The spatial resolution study examined the ability for each mission configuration to recover a static mass signal within a small block of $1^{\circ} \times 1^{\circ}$. This study showed that even without the presence of temporal aliasing there was still no improvement in the gravity estimate for GFO Case 1 as compared to GRACE. This means that in order to take advantage of the improved range-rate accuracy provided by the laser, the errors associated with the on-board accelerometers need to be reduced. In the case where the 
drag-free system is implemented (GFO Case 2 and GFO Case 3), the accuracy of the results are significantly affected by the level of noise in the range-rate measurements, with the laser allowing for a much more spatially focused estimate.

A final interesting result of this work, shows that the mascon estimation method provides large improvements for estimating mass changes within smaller basins, as compared to estimates formed by applying an unconstrained averaging kernel to monthly sets of spherical harmonics. For large basins, the accuracy of the mass change estimates are similar. The improved estimates of the mascon approach is due to the spatial constraints which are applied to the mascons, and the increase in leakage error that exists for smaller averaging kernels. It is expected that a similar constraining procedure could be applied to spherical harmonic estimates to achieve similar results.

\subsection{Recommendations}

In regards to a GRACE Follow-On mission, the most important priority should be a continuation of the time series of data. As discussed in Chapter 1, the data provided by the GRACE mission has made a great deal of unique contributions to a number of important areas of scientific research, so an interruption in the data is clearly undesirable. The results presented in this work reveal that simply replacing the K-band microwave ranging system with the laser interferometer would not provide improvements to the gravity estimation for the current level of accelerometer error and the current GRACE orbit. To achieve better performance with a GFO, the improved laser ranging system should be accompanied by a reduction in the errors associated with the accelerometer. A mission that is equipped with both a laser interferometer and a drag-free system would be considerably more expensive, and so is unlikely to fly in the immediate future. If only one of the new technologies investigated here were 
to be incorporated into a follow-on mission, the most benefit would be realized with a drag-free system, and would ideally be placed in a lower altitude orbit than the current GRACE mission.

Even if it were realistic to launch a GRACE Follow-On mission equipped with both the laser interferometer and a drag-free system, this report shows that the improvement to regional gravity estimates would still only be moderate, due to the limitations of temporal aliasing. It is recommended here, that future research in regards to a GFO should focus on ways in which the effects of temporal aliasing can be reduced. It is conceivable that improved methods of data processing, such as a method which analyzes the data along-track, might effectively improve the temporal resolution of the solutions, thus reducing this error. Additionally, alternative architectures for a follow-on mission might be investigated to determine if different mission configurations, such as some sort of satellite constellation, would reduce the aliasing errors. Lastly, more accurate geophysical models would also decrease the temporal aliasing errors. This reduction in aliasing errors would result in better gravity estimates for a GFO mission, with the updated technologies, than are observed in this simulation study. 


\section{Bibliography}

Barletta, V. R., and A. Bordoni, Clearing observed PGR in GRACE data aimed at global viscosity inversion: Weighted Mass Trends technique, Geophysical Research Letters, 36, L02305, doi:10.1029/2008GL036429, 2009.

Bender, P. L., R. S. Nerem, and J. M. Wahr, Possible future use of laser gravity gradiometers, Space Science Reviews, 108, 385-392, 2003.

Bettadpur, S., private communication, 2007.

Bingham, R. J., and K. Haines, Mean dynamic topography: intercomparisons and errors, Phil. Trans. R. Soc. A, 364, 903-916, doi:10.1098/rsta.2006.1745, 2006.

Bingham, R. J., and C. W. Hughes, Observing seasonal bottom pressure variability in the north pacific with grace, 33, L08607, doi:10.1029/2005GL025489, 2006.

Birol, F., J. M. Brankart, J. M. Lemoine, P. Brasseur, and J. Verron, Assimilation of satellite altimetry referenced to the new GRACE geoid estimate, Geophysical Research Letters, 32, L06601, doi:10.1029/2004GL021329, 2005.

Briggs, W. L., The DFT: An Owner's Manual for the Discrete Fourier Tranform, Society for Inudstrial and Applied Mathematics, Philadelphia, 1995.

Carrère, L., and F. Lyard, Modeling the barotropic response of the global ocean to atmospheric wind and pressure forcing - comparison with observations, Geophysical Research Letters, 30 (6), 1275-1278, doi:10.1029/2002GL016473, 2003.

Castruccio, F., J. Verron, L. Gourdeau, J. M. Brankart, and P. Brasseur, On the role of GRACE mission in the joint assimilation of altimetric and TAO data in a tropical Pacific Ocean model, Geophysical Research Letters, 33, L14616, doi:10.1029/2006GL025823, 2006.

Cazenave, A., and R. S. Nerem, Present-day sea level change: Observations and causes, Review of Geophysics, 42, RG3001, doi:10.1029/2003RG000139, 2004.

Chambers, D. P., Evaluation of new GRACE time-variable gravity data over the ocean, Geophysical Research Letters, 33, L17603, doi:10.1029/2006GL027296, 2006a. 
Chambers, D. P., Observing seasonal steric sea level variations with GRACE and satellite altimetry, Journal of Geophysical Research, 111, C03010, doi:10.1029/2005JC002914, 2006b.

Chambers, D. P., J. Wahr, and R. S. Nerem, Preliminary observations of global ocean mass variations with GRACE, Geophysical Research Letters, 31, L13310, doi:10.1029/2004GL020461, 2004.

Chen, J. L., C. R. Wilson, B. D. Tapley, J. S. Famiglietti, and M. Rodell, Seasonal global mean sea level change from satellite altimeter, GRACE, and geophysical models, Journal of Geodynamics, 79, 532-539, 2005.

Chen, J. L., C. R. Wilson, D. D. Blankenship, and B. D. Tapley, Antarctic mass rates from GRACE, Geophysical Research Letters, 33, L11502, doi:10.1029/2006GL026369, 2006a.

Chen, J. L., C. R. Wilson, and B. D. Tapley, Satellite Gravity Measurements Confirm Accelerated Melting of Greenland Ice Sheet, Science, 313, 1958-1960, doi:10.1126/science.1129,007, 2006b.

Chen, J. L., C. R. Wilson, B. D. Tapley, D. D. Blankenship, and E. R. Ivins, Patagonia Icefield melting observed by Gravity Recovery and Climate Experiment (GRACE), Geophysical Research Letters, 34, L22501, doi:10.1029/2007GL031871, 2007.

Crowley, J. W., J. X. Mitrovica, R. C. Bailey, M. E. Tamisiea, and J. L. Davis, Land water storage within the Congo Basin inferred from GRACE satellite gravity data, Geophysical Research Letters, 33, L19402, doi:10.1029/2006GL027070, 2006.

Dehne, M., F. G. Cervantes, B. Sheard, G. Heinzel, and K. Danzmann, Laser interferometer for spaceborne mapping of the earth's gravity field, Journal of Physics: Conference Series, 154, doi:10.1008/1742-6596/154/1/012,023, 2009.

Desai, S. D., and D. N. Yuan, Applications of the convolution formalism to the ocean tide potential: Results from the Gravity Recovery and Climate Experiment (GRACE), Journal of Geophysical Research, 111, C06023, doi:10.1029/2005JC003,361, 2006.

Ebisuzaki, W., A method to estimate the statistical significance of a correlation when the data are serially correlated, Journal of Climate, 10 (9), 2147-2153, 1997.

editors, M.-W. (Ed.), Merriam-Webster's Collegiate Dictionary: 11th Edition, MerriamWebster, Inc., Springfield, MA, 2003.

Encarnacao, J., R. Klees, E. Zapreeva, P. Ditmar, and J. Kusche, Influence of hydrologyrelated temporal aliasing on the quality of monthly derived from grace satellite gravimetric data, International Association of Geodesy Symposia, 133, 323-328, 2009.

Flechtner, F., Aod1b product description document for product releases 01 to 04, grace 327-750 (gr-gfz-aod-0001), 2007. 
Gray, R. M., and J. W. Goodman, Fourier Transforms, Kluwer Academic Publishers, Boston, 1995.

Han, S.-C., and C. K. Shum, GRACE observations of M2 and S2 ocean tides underneath the Filchner-Ronne and Larsen ice shelves, Antarctica, Geophysical Research Letters, 32, L20311, doi:10.1029/2005GL024296, 2005.

Han, S.-C., C. Jekeli, and C. K. Shum, Time-variable aliasing effects of ocean tides, atmosphere, and continental water mass on monthly mean GRACE gravity field, Journal of Geophysical Research, 109, B04403, doi:10.1029/2003JB002501, 2004.

Han, S.-C., C. K. Shum, C. Jekeli, and D. Alsdorf, Improved estimation of terrestrial water storage changes from GRACE, Geophysical Research Letters, 32, L07302, doi:10.1029/2005GL022382, 2005.

Han, S.-C., R. D. Ray, and S. B. Luthcke, Ocean tidal solutions in Antarctica from GRACE inter-satellite tracking data, Geophysical Research Letters, 34, L21607, doi:10.1029/2007GL031540, 2007.

Han, S.-C., J. Sauber, S. B. Luthcke, C. Ji, and F. F. Pollitz, Implications of postseismic gravity change following the great 2004 Sumatra-Andaman earthquake from the regional harmonic analysis of GRACE intersatellite tracking data, Journal of Geophysical Research, 113, B11413, doi:10.1029/2008JB005705, 2008.

Hill, G. W., Researches in lunar theory, Americal Journal of Mathematics, 1, 5-26, 1878.

Jayne, S. R., Circulation of the North Atlantic Ocean from altimetry and the Gravity Recovery and Climate Experiment geoid, Journal of Geophysical Research, 111, C03005, doi:10.1029/2005JC003128, 2006.

Jayne, S. R., J. M. Wahr, and F. O. Bryan, Observing ocean heat content using satellite gravity and altimetry, Journal of Geophysical Research, 108 (C2), 3031, doi:10.1029/2002JC001619, 2003.

Jekeli, C., Alternative methods to smooth the Earth's gravity field, Department of geodetic science, report no. 32\%, The Ohio State University, 1981.

Kang, Z., B. Tapley, S. Bettadpur, J. Ries, and P. Nagel, Precise orbit determination for grace using accelerometer data, Advances in Space Research, 38, 2131-2136, 2006.

Kaula, W. M., Theory of Satellite Geodesy, Dover Publications, Inc., Mineola, New York, 2000.

Kim, J.-R., Simulation study of a low-low satellite-to-satellite tracking mission, Ph.D. thesis, University of Texas at Austin, 2000. 
Lefèvre, F., F. Lyard, C. LeProvost, and E. Schrama, FES99: a global tide finite element solution assimilating tide guage and altimetric information, Journal of Atmosphere and Ocean Technology, 19, 1345-1356, 2002.

Luthcke, S., private communication, 2009.

Luthcke, S. B., H. J. Zwally, W. Abdalati, D. D. Rowlands, R. D. Ray, R. S. Nerem, F. G. Lemoine, J. J. McCarthy, and D. S. Chinn, Recent greenland ice mass loss by drainage system from satellite gravity observations, Science, 314 (5803), 1286-1289, doi:10.1126/science.1130776, 2006.

Luthcke, S. B., A. A. Arendt, D. D. Rowlands, J. J. McCarthy, and C. F. Larsen, Recent glacier mass changes in the Gulf of Alaska region from GRACE mascon solutions, Journal of Glaciology, 54 (188), 767-777, 2008.

Marchetti, P., J. J. Blandino, and M. A. Demetriou, Electric Propulsion and Controller Design for Drag-Free Spacecraft Operation, Journal of Spacecraft and Rockets, 45 (6), 1303-1315, doi:10.2514/1.36307, 2008.

Moore, P., and M. A. King, Antarctic ice mass balance estimates from GRACE: Tidal aliasing effects, Journal of Geophysical Research, 113, F02005, doi:10.1029/2007JF000871, 2008.

Moulton, F. R., Introduction to Celestial Mechanics, MacMillan Company, New York, 1914.

Muller, P. M., and W. L. Sjogren, Mascons: Lunar mass concentrations, Science, 161, 680-684, 1968.

Nastula, J., R. M. Ponte, and D. A. Salstein, Comparison of polar motion excitation series derived from GRACE and from analysis of geophysical fluids, Geophysical Research Letters, 34, L11306, doi:10.1029/2006GL028983, 2007.

Nerem, R. S., J. M. Wahr, and E. W. Leuliette, Measuring the distribution of ocean mass using GRACE, Space Science Reviews, 108, 331-344, 2003.

Nerem, R. S., P. Bender, B. Loomis, M. M. Watkins, W. M. Folkner, M. Stephens, R. Craig, J. Leitch, and R. Pierce, Developmnet of an interferometric laser ranging system for a follow-On gravity mission to GRACE, Eos Trans. AGU, 87 (52), Fall Meet. Suppl. Abstract G11B-02, 2006.

Niu, G.-Y., K.-W. Seo, Z.-L. Yang, C. Wilson, H. Su, J. Chen, and M. Rodell, Retrieving snow mass from GRACE terrestrial water storage change with a land surface model, Geophysical Research Letters, 34, L15704, doi:10.1029/2007GL030413, 2007.

Pierce, R., J. Leitch, M. Stephens, P. Bender, and R. Nerem, Intersatellite range monitoring using optical interferometry, Applied Optics, 47 (27), 5007-5018, 2008. 
Ponte, R. M., K. J. Quinn, C. Wunsch, and P. Heimbach, A comparison of model and GRACE estimates of the large-scale seasonal cycle in ocean bottom pressure, Geophysical Research Letters, 34, L09603, doi:10.1029/2007GL029599, 2007.

Ramillien, G., F. Frappart, A. Cazenave, and A. Güntner, Time variations of land water storage from an inversion of 2 years of GRACE geoids, Earth and Planetary Science Letters, 235, 283-301, 2005.

Ramillien, G., A. Lombard, A. Cazenave, R. R. Ivins, M. Llubes, F. Remy, and R. Biancale, Interannual variations of the mass balance of the antarctica and greenland ice sheets from grace, Global and Planetary Change, 53 (3), 198-208, 2006.

Ray, R. D., A global ocean tide model from TOPEX/POSEIDON altimetry: GOT99, NASA technical memorandum 209478, Goddard Space Flight Center, 1999.

Ray, R. D., and S. B. Luthcke, Tidal model errors and grace gravimetry: towards a more realistic assessment, Geophysical Journal International, 167, 1055-1059, 2006.

Rodell, M., and J. S. Famiglietti, Detectability of variations in continental water storage from satellite observations of the time dependent gravity field, Water Resources Research, 35 (9), 2705-2723, 1999.

Rodell, M., J. S. Famiglietti, J. Chen, S. I. Seneviratne, P. Viterbo, S. Holl, and C. R. Wilson, Basin scale estimates of evapotranspiration using GRACE and other observations, Geophysical Research Letters, 31, L20504, doi:10.1029/2004GL020873, 2004a.

Rodell, M., et al., The Global Land Data Assimilation System, Bulletin of the American Meteorological Society, 85, 381-394, 2004b.

Rowlands, D. D., R. D. Ray, D. S. Chinn, and F. G. Lemoine, Short-arc analysis of intersatellite tracking data in a gravity mapping mission, Journal of Geodesy, 76, 307-316, 2002.

Rowlands, D. D., S. B. Luthcke, S. Klosko, F. G. Lemoine, D. S. Chinn, J. J. McCarthy, C. M. Cox, and O. B. Anderson, Resolving mass flux at high spatial and temporal resolution using GRACE intersatellite measurements, Geophysical Research Letters, 32, L04310, doi:10.1029/2004GL021908, 2005.

Seeber, G., Satellite Geodesy: 2nd Edition, Walter de Gruyter, Berlin, 2003.

Seo, K. W., and C. R. Wilson, Estimating grace aliasing errors, International Association of Geodesy Symposia, 129, 339-345: doi:10.1007/b138327, 2005.

Seo, K. W., C. R. Wilson, J. Chen, and D. E. Waliser, Grace's spatial aliasing error, Geophys. J. Int., 172, 41-48, doi:10.1111/j.1365-246X.2007.03611.x, 2008a.

Seo, K. W., C. R. Wilson, S. C. Han, and D. E. Waliser, Gravity recovery and climate experiment (grace) alias error from ocean tides, Journal of Geophysical Research, 113, B03405, doi:10.1029/2006JB004747, 2008b. 
Shin, Y. H., C.-K. Shum, C. Braitenberg, S. M. Lee, H. Xu, K. S. Choi, J. H. Baek, and J. U. Park, Three-dimensional fold structure of the Tibetan Moho from GRACE gravity data, Geophysical Research Letters, 36, L01302, doi:10.1029/2008GL036068, 2009 .

Strassberg, G., B. R. Scanlon, and M. Rodell, Comparison of seasonal terrestrial water storage variations from GRACE with groundwater-level measurements from the High Plains Aquifer (USA), Geophysical Research Letters, 34, L14402, doi:10.1029/2007GL030139, 2007.

Swenson, S., and P. C. D. Milly, Climate model biases in seasonality of continental water storage revealed by satellite gravimetry, Water Resources Research, 42, W03201, doi:10.1029/2005WR004628, 2006.

Swenson, S., and J. Wahr, Methods for inferring regional surface-mass anomalies from Gravity Recovery and Climate Experiment (GRACE) measurements of time-variable gravity, Journal of Geophysical Research, $10^{` 7}$ (B9), 2193, doi:10.1029/2001JB000576, 2002 .

Swenson, S., and J. Wahr, Estimating large-scale precipitation minus evapotranspiration from GRACE satellite gravity measurements, Journal of Hydrometerology, 7, 252-270, doi:10.1175/JHM478.1, 2006a.

Swenson, S., and J. Wahr, Post-processing removal of correlated errors in GRACE Data, Geophysical Research Letters, 33, L08402, doi:10.1029/2005GL025285, 2006b.

Swenson, S., P. J.-F. Yeh, J. Wahr, and J. Famiglietti, A comparison of terrestrial water storage variations from GRACE with in situ measurements from Illinois, Geophysical Research Letters, 33, L16401, doi:10.1029/2006GL026962, 2006.

Swenson, S., D. Chambers, and J. Wahr, Estimating geocenter variations from a combination of GRACE and ocean model output, Journal of Geophysical Research, 113, B08410, doi:10.1029/2007JB005338, 2008a.

Swenson, S., J. Famiglietti, J. Basara, and J. Wahr, Estimating profile soil moisture and groundwater variations using GRACE and Oklahoma Mesonet soil moisture data, Water Resources Research, 44, W01413, doi:10.1029/2007WR006057, 2008b.

Syed, T. H., J. S. Famiglietti, J. Chen, M. Rodell, S. I. Seneviratne, P. Viterbo, and C. R. Wilson, Total basin discharge for the Amazon and Mississippi River basins from GRACE and a land-atmosphere water balance, Geophysical Research Letters, 32, L24404, doi:10.1029/2005GL024851, 2005.

Syed, T. H., J. S. Famiglietti, M. Rodell, J. Chen, and C. R. Wilson, Analaysis of terrestrial water storage changes from GRACE and GLDAS, Water Resources Research, 44, W02433, doi:10.1029/2006WR005779, 2008. 
Tapley, B. D., D. P. chamber, S. Bettadpur, and J. C. Ries, Large scale ocean circulation from the GRACE GGM01 Geoid, Geophysical Research Letters, 30 (22), 2163, doi:10.1029/2003GL018622, 2003.

Tapley, B. D., S. Bettadpur, J. C. Ries, P. F. Thompson, and M. M. Watkins, GRACE measurements of mass variability in the Earth system, Science, 305, 503-505, 2004a.

Tapley, B. D., S. Bettadpur, M. Watkins, and C. Reigber, The Gravity Recovery and Climate Experiment: Mission overview and early results, Geophysical Research Letters, 31, L24806, doi:10.1029/2004GL021220, 2004b.

Tapley, B. D., B. E. Schutz, and G. H. Born, Statistical Orbit Determination, Elsevier Inc., San Diego, 2004c.

Thompson, P. F., S. V. Bettadpur, and B. D. Tapley, Impact of short period, nontidal, temporal mass variability on GRACE gravity estimates, Geophysical Research Letters, 31, L06619, doi:10.1029/2003GL019285, 2004.

Torge, W., Geodesy: 3rd Edition, Walter de Gruyter, Berlin, 2001.

Vallado, D. A., Fundamentals of Astrodynamics and Applications: Second Edition, Microcosm Press, El Segundo, California, 2001.

van Dam, T., J. Wahr, and D. Lavallee, A comparison of annual vertical crustal displacements from GPS and Gravity Recovery and Climate Experiment (GRACE) over Europe, Journal of Geophysical Research, 112, B03404, doi:10.1029/2006JB004335, 2007.

Velicogna, I., and J. Wahr, Postglacial rebound and Earth's viscosity structure from GRACE, Journal of Geophysical Research, 107 (B12), 2376, doi:10.1029/2001JB001735, 2002.

Velicogna, I., and J. Wahr, Greenland mass balance from GRACE, Geophysical Research Letters, 32, L18505, doi:10.1029/2005GL023955, 2005.

Velicogna, I., and J. Wahr, Acceleration of greenland ice mass loss in spring 2004, Nature, 443, 329-331, doi:10.1038/nature05,168, 2006a.

Velicogna, I., and J. Wahr, Measurements of time-variable gravity show mass loss in Antarctica, Science, 311, 1754-1756, 2006b.

Velicogna, I., J. Wahr, E. Hanna, and P. Huybrechts, Short term mass variability in Greenland, from GRACE, Geophysical Research Letters, 32, L05501, doi:10.1029/2004GL021948, 2005.

Wahr, J., M. Molenaar, and F. Bryan, Time variability of the Earth's gravity field: Hydrological and oceanic effects and their possible detection using GRACE, Journal of Geophysical Research, 103 (B12), 30,205-30,229, 1998. 
Wahr, J., S. Swenson, V. Zlotnicki, and I. Velicogna, Time-variable gravity from GRACE: First results, Geophysical Research Letters, 31, L11501, doi:10.1029/2004GL019779, 2004.

Wahr, J., S. Swenson, and I. Velicogna, Accuracy of GRACE mass estimates, Geophysical Research Letters, 33, L06401, doi:10.1029/2005GL025305, 2006.

Walker, J. S., Fast Fourier Transforms, CRC Press Inc., Boca Raton, FL, 1991.

Wiese, D. N., W. M. Folkner, and R. S. Nerem, Alternative mission architectures for a gravity recovery satellite mission, Journal of Geodesy, pp. doi:10.1007/s00190-0080274-1, 2008.

Wong, L., G. Buechler, W. Downs, W. Sjogren, P. Puller, and P. Gottlieb, A surfacelayer representation of the lunar gravitational field, Journal of Geophysical Research, 76 (26), 6,220-6,236, 1971.

Wouters, B., D. Chambers, and E. J. O. Schrama, GRACE observes small-scale mass loss in Greenland, Geophysical Research Letters, 35, L20501, doi:10.1029/2008GL034816, 2008.

Wunsch, C., and D. Stammer, Atmospherical loading and the oceanic 'inverted barometer' effect, Review of Geophysics, 35 (1), 79-107, 1997. 


\section{Appendix A}

\section{Spherical Harmonics}

As shown in Chapter 2 the gravitational potential at a point outside of the Earth is given by,

$$
V=\frac{\mu}{r}\left(1+\sum_{n=1}^{\infty} \sum_{m=0}^{n}\left(\frac{R_{\oplus}}{r}\right)^{n} P_{n m}(\sin \phi)\left(C_{n m} \cos m \lambda+S_{n m} \sin m \lambda\right)\right) .
$$

The terms $C_{n m}$, and $S_{n m}$ are called geopotential coefficients and are defined by,

$$
\begin{array}{cc}
C_{n}=\frac{1}{M} \iiint_{\text {earth }}\left(\frac{r^{\prime}}{R_{\oplus}}\right)^{n} P_{n m}(\sin \phi) d m & \text { for } m=0 \\
\left\{\begin{array}{c}
C_{n m} \\
S_{n m}
\end{array}\right\}=\frac{2}{M} \times \frac{(n-m) !}{(n+m) !} \iiint_{\text {earth }}\left(\frac{r^{\prime}}{R_{\oplus}}\right)^{n} P_{n m}(\sin \phi)\left\{\begin{array}{c}
\cos m \lambda^{\prime} \\
\sin m \lambda^{\prime}
\end{array}\right\} d m & \text { for } m \neq 0
\end{array}
$$

where $P_{n m}(\sin \phi)$ are the associated Legendre functions. The associated Legendre functions are given by,

$$
P_{n m}(x)=\left(1-x^{2}\right)^{\frac{m}{2}} \frac{d^{m}}{d x^{m}} P_{n}(x)
$$

where,

$$
P_{n}(x)=\frac{1}{2^{n} n !} \frac{d^{n}}{d x^{n}}\left[\left(x^{2}-1\right)^{n}\right] .
$$

As explained in Section 2.3 the fully normalized associated Legendre functions are used. For the fully normalized definition of the associated Legendre functions the 
following relation is satisfied,

$$
\frac{1}{4 \pi} \iint_{\sigma}\left(\tilde{P}_{n m}\left\{\begin{array}{c}
\cos m \lambda \\
\sin m \lambda
\end{array}\right\}\right)^{2} d \sigma=1
$$

where $\sigma$ is the unit sphere. The fully normalized associate Legendre functions are then given by,

$$
\tilde{P}_{n m}(\sin \phi)=\left[\frac{k(2 n+1)(n-m) !}{(n+m) !}\right]^{1 / 2} P_{n m}(\sin \phi) .
$$

with $k=1$ for $m=0$, and $k=2$ for $m \neq 0$.

It is useful to describe spherical harmonics in terms of their nodal lines. In the direction of longitude the number of nodal lines is the number of zeros from $\lambda=0$ to $\lambda=2 \pi$ and for latitude it is the number of zeros from $\phi=\frac{-\pi}{2}$ to $\phi=\frac{\pi}{2}$. Figure A.1 shows examples of the three types of spherical harmonics.
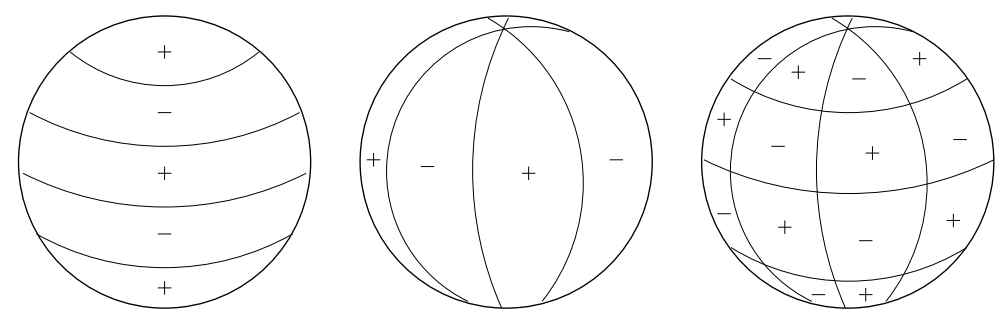

Figure A.1: Examples of harmonic types: (i)Zonal (ii)Sectorial (iii)Tesseral.

The harmonic types have the following properties:

(i) Zonal harmonics occur when $m=0$. For this case there are no nodal lines of longitude and there are $n$ nodal lines of latitude.

(ii) Sectorial harmonics occur when $n=m$. There are no nodal lines of latitude and there are $2 n$ nodal lines of longitude.

(iii) Tesseral harmonics occur when $n \neq m$. In this case there are $2 m$ nodal lines of longitude and there are $n-m$ nodal lines of latitude. 
The equations required for orbit propagation and parameter estimation with regards to the spherical harmonic parameters are now presented. This material is developed in [Vallado, 2001]. As described by Eq. (3.3), the acceleration due to the non-sphericity of the Earth is equal to the gradient of the potential $U$, which is the non-spherical component of Eq. (A.1) and was previously defined in Eq. (3.4). It is convenient to differentiate the Legendre functions in spherical coordinates $(r, \phi, \lambda)$ and to determine the acceleration in Cartesian coordinates, so the chain rule is applied. Matrix differentiation is done in the body-fixed frame and numerical integration is performed in an inertial frame. The position vector is then defined as $\mathbf{r}=x \hat{i}+y \hat{j}+z \hat{k}$ in the body-fixed frame. The acceleration is:

$$
\ddot{\mathbf{r}}=\frac{\partial U}{\partial \mathbf{r}}=\frac{\partial U}{\partial r} \frac{\partial r}{\partial \mathbf{r}}+\frac{\partial U}{\partial \phi} \frac{\partial \phi}{\partial \mathbf{r}}+\frac{\partial U}{\partial \lambda} \frac{\partial \lambda}{\partial \mathbf{r}}
$$

Evaluating the partial derivatives in Eq. (A.7) gives:

$$
\begin{aligned}
& \frac{\partial U}{\partial r}=-\frac{\mu}{r^{2}} \sum_{n=2}^{\infty} \sum_{m=0}^{n}\left(\frac{R_{\oplus}}{r}\right)^{n}(n+1) P_{n, m}(\sin \phi)\left(C_{n m} \cos m \lambda+S_{n m} \sin m \lambda\right) \\
& \frac{\partial U}{\partial \phi}=\frac{\mu}{r} \sum_{n=2}^{\infty} \sum_{m=0}^{n}\left(\frac{R_{\oplus}}{r}\right)^{n}\left(P_{n, m+1}(\sin \phi)-m \tan \phi P_{n, m}(\sin \phi)\right)\left(C_{n m} \cos m \lambda+S_{n m} \sin m \lambda\right) \\
& \frac{\partial U}{\partial \lambda}=\frac{\mu}{r} \sum_{n=2}^{\infty} \sum_{m=0}^{n}\left(\frac{R_{\oplus}}{r}\right)^{n} m P_{n, m}(\sin \phi)\left(S_{n m} \cos m \lambda-C_{n m} \sin m \lambda\right)
\end{aligned}
$$

The partial derivatives of the spherical coordinates are:

$$
\begin{aligned}
& \frac{\partial r}{\partial \mathbf{r}}=\frac{\mathbf{r}^{T}}{r} \\
& \frac{\partial \phi}{\partial \mathbf{r}}=\frac{1}{\sqrt{x^{2}+y^{2}}}\left(-\frac{\mathbf{r}^{T} z}{r^{2}}+\frac{\partial z}{\partial \mathbf{r}}\right) \\
& \frac{\partial \lambda}{\partial \mathbf{r}}=\frac{1}{x^{2}+y^{2}}\left(x \frac{\partial y}{\partial \mathbf{r}}-y \frac{\partial x}{\partial \mathbf{r}}\right)
\end{aligned}
$$

Combining the partial derivatives and separating them into components gives the ac- 
celerations in Cartesian coordinates:

$$
\begin{aligned}
& \ddot{x}=\left\{\frac{1}{r} \frac{\partial U}{\partial r}-\frac{z}{r^{2} \sqrt{x^{2}+y^{2}}} \frac{\partial U}{\partial \phi}\right\} x-\left\{\frac{1}{x^{2}+y^{2}} \frac{\partial U}{\partial \lambda}\right\} y \\
& \ddot{y}=\left\{\frac{1}{r} \frac{\partial U}{\partial r}-\frac{z}{r^{2} \sqrt{x^{2}+y^{2}}} \frac{\partial U}{\partial \phi}\right\} y+\left\{\frac{1}{x^{2}+y^{2}} \frac{\partial U}{\partial \lambda}\right\} x \\
& \ddot{z}=\frac{1}{r} \frac{\partial U}{\partial r} z+\frac{\sqrt{x^{2}+y^{2}}}{r^{2}} \frac{\partial U}{\partial \phi}
\end{aligned}
$$

Eq. (A.10) describes the term $\mathbf{f}_{N S}$ as given in Eq. (3.3) and is used for satellite orbit propagation in the presence of a non-spherical gravitational potential described by spherical harmonics.

The partial derivatives of the acceleration vector with respect to the position vector are contained in the $A$ matrix of Eq. (3.17) and are given here:

$$
\begin{aligned}
\frac{\partial \ddot{\mathbf{r}}}{\partial \mathbf{r}} & =\frac{\partial}{\partial \mathbf{r}}\left(\frac{\partial U}{\partial r}\right) \frac{\partial r}{\partial \mathbf{r}}+\frac{\partial}{\partial \mathbf{r}}\left(\frac{\partial U}{\partial \phi}\right) \frac{\partial \phi}{\partial \mathbf{r}}+\frac{\partial}{\partial \mathbf{r}}\left(\frac{\partial U}{\partial \lambda}\right) \frac{\partial \lambda}{\partial \mathbf{r}} \\
& +\frac{\partial U}{\partial r} \frac{\partial^{2} r}{\partial \mathbf{r}^{2}}+\frac{\partial U}{\partial r} \frac{\partial^{2} \phi}{\partial \mathbf{r}^{2}}+\frac{\partial U}{\partial r} \frac{\partial^{2} \lambda}{\partial \mathbf{r}^{2}}
\end{aligned}
$$


The components of Eq. (A.11) are computed:

$$
\begin{aligned}
\frac{\partial^{2} U}{\partial r^{2}} & =\frac{\mu}{r^{3}} \sum_{n=2}^{\infty}\left(\frac{R_{\oplus}}{r}\right)^{n}(n+2)(n+1) \sum_{m=0}^{n} P_{n, m}(\sin \phi)\left(C_{n m} \cos m \lambda+S_{n m} \sin m \lambda\right) \\
\frac{\partial^{2} U}{\partial r \partial \phi} & =\frac{\partial^{2} U}{\partial \phi \partial r}=-\frac{\mu}{r^{2}} \sum_{n=2}^{\infty}\left(\frac{R_{\oplus}}{r}\right)^{n}(n+1) \sum_{m=0}^{n}\left(P_{n, m+1}(\sin \phi)-m \tan \phi P_{n, m}(\sin \phi)\right) \\
& \times\left(C_{n m} \cos m \lambda+S_{n m} \sin m \lambda\right) \\
\frac{\partial^{2} U}{\partial r \partial \lambda} & =\frac{\partial^{2} U}{\partial \lambda \partial r}=-\frac{\mu}{r^{3}} \sum_{n=2}^{\infty}\left(\frac{R_{\oplus}}{r}\right)^{n}(n+1) \sum_{m=0}^{n} m P_{n, m}(\sin \phi)\left(S_{n m} \cos m \lambda-C_{n m} \sin m \lambda\right) \\
\frac{\partial^{2} U}{\partial \phi^{2}} & =\frac{\mu}{r} \sum_{n=2}^{\infty}\left(\frac{R_{\oplus}}{r}\right)^{n} \sum_{m=0}^{n}\left(C_{n m} \cos m \lambda+S_{n m} \sin m \lambda\right)\left(\tan \phi P_{n, m+1}(\sin \phi)\right) \\
& +\left(m^{2} \sec ^{2} \phi-m \tan ^{2} \phi-n(n+1) P_{n, m}(\sin \phi)\right) \\
\frac{\partial^{2} U}{\partial \phi \partial \lambda} & =\frac{\partial^{2} U}{\partial \lambda \partial \phi} \\
& =\frac{\mu}{r} \sum_{n=2}^{\infty}\left(\frac{R_{\oplus}}{r}\right)^{n} \sum_{m=0}^{n} m\left(S_{n m} \cos m \lambda-C_{n m} \sin m \lambda\right)\left(P_{n, m}(\sin \phi)-m \tan \phi P_{n, m}(\sin \phi)\right) \\
\frac{\partial^{2} U}{\partial \lambda^{2}} & =-\frac{\mu}{r} \sum_{n=2}^{\infty}\left(\frac{R_{\oplus}}{r}\right)^{n} \sum_{m=0}^{n} m^{2} P_{n, m} \sin \phi\left(C_{n m} \cos m \lambda+S_{n m} \sin m \lambda\right)
\end{aligned}
$$

The second derivatives of the spherical coordinates are:

$$
\begin{aligned}
\frac{\partial^{2} r}{\partial \mathbf{r}^{2}} & =\frac{1}{r}\left[\mathbf{I}-\frac{\mathbf{r r}^{T}}{r^{2}}\right] \\
\frac{\partial^{2} \phi}{\partial \mathbf{r}^{2}} & =\frac{1}{\left(x^{2}+y^{2}\right)^{3 / 2}}\left[\left(\frac{\partial z}{\partial \mathbf{r}}\right)^{T}-\frac{z \mathbf{r}}{r^{2}}\right]\left[x\left(\frac{\partial x}{\partial \mathbf{r}}\right)+y\left(\frac{\partial y}{\partial \mathbf{r}}\right)\right] \\
& -\frac{1}{r^{2} \sqrt{x^{2}+y^{2}}}\left[\mathbf{r}\left(\frac{\partial z}{\partial \mathbf{r}}\right)+z \mathbf{I}-\frac{2 z}{r^{2}} \mathbf{r r}^{T}\right] \\
\frac{\partial^{2} \lambda}{\partial \mathbf{r}^{2}} & =-\frac{2}{\left(x^{2}+y^{2}\right)}\left[\begin{array}{c}
-y \\
0
\end{array}\right]\left[x\left(\frac{\partial x}{\partial \mathbf{r}}\right)+y\left(\frac{\partial y}{\partial \mathbf{r}}\right)\right]+\frac{1}{\left(x^{2}+y^{2}\right)}\left[\begin{array}{ccc}
0 & -1 & 0 \\
1 & 0 & 0 \\
0 & 0 & 0
\end{array}\right]
\end{aligned}
$$

where $\mathbf{I}$ is the identity matrix in the body-fixed frame.

Gravity field solutions are often described in terms of their spatial resolution. In degrees the spatial resolution is defined by $180^{\circ} / N_{\max }$ where $N_{\max }$ is the spherical 
harmonic degree at which the expansion is truncated. The spatial resolution given in kilometers is defined by,

$$
\text { spatial resolution }=\frac{40,000 \mathrm{~km}}{2 N_{\max }} .
$$

It is common to describe a spherical harmonic representation of the gravity field as having a certain level of accuracy (in terms of geoid height or equivalent water height) to some spatial resolution. 


\section{Appendix B}

\section{Fourier Transforms}

Definitions are given here for various Fourier transforms. The discrete inverse Fourier transform was used for generating a time series of range-rate errors from the spectral density of errors as discussed in Section 4.2.2. The work in this section is presented in greater detail in [Briggs, 1995; Gray and Goodman, 1995; Walker, 1991].

The continuous Fourier transform $X(f)$ of a function of time $x(t)$ is defined as,

$$
X(f)=\int_{-\infty}^{\infty} x(t) e^{-i 2 \pi f t} d t
$$

where $t$ is time and $f$ is frequency. The unit of time is seconds and the unit of frequency is Hz. The inverse Fourier transform is then given by,

$$
x(t)=\int_{-\infty}^{\infty} X(f) e^{i 2 \pi f t} d f
$$

For this study a discrete function of time, $x[n]$, is required. The discrete Fourier transform $X[l]$ is given by,

$$
X[l]=\sum_{n=0}^{N-1} x[n] e^{-i 2 \pi l n / N} \quad l=0,1,2, \ldots, N-1
$$

and its inverse is,

$$
x[n]=\frac{1}{N} \sum_{l=0}^{N-1} X[l] e^{i 2 \pi l n / N} \quad n=0,1,2, \ldots, N-1
$$


where $N$ is the number of discrete data points in the time series.

The number of data points in the time series is defined by the time period, $T$, and the sampling rate in the time domain, $\Delta t$,

$$
N=\frac{T}{\Delta t}
$$

The time series signal is defined at the discrete points,

$$
t_{n}=n \Delta t, \quad n=0,1,2, \ldots, N-1
$$

The frequency sampling rate is defined by,

$$
\Delta f=\frac{1}{N \Delta t}
$$

and the frequency spectrum is defined by,

$$
f_{l}=\frac{l}{N \Delta t}, \quad l=-\frac{N}{2}, \ldots, \frac{N}{2}
$$

The inverse discrete Fourier transform was performed using the MATLAB software package which uses the IFFT (inverse fast Fourier transform) algorithm. For the IFFT algorithm the summation takes place over $l=0, \ldots, N-1$ as in Equation B.4. For this reason the frequency spectrum given in Equation B.8 is rearranged as follows:

$$
f_{l}=\left\{0, \frac{1}{N \Delta t}, \ldots, \frac{N / 2-1}{N \Delta t}, \frac{1}{2 \Delta t},-\frac{N / 2-1}{N \Delta t}, \ldots,-\frac{1}{N \Delta t}\right\} .
$$

In order to introduce random phases into the spectral density prior to taking the inverse discrete Fourier transform, the positive frequency points were multiplied by $e^{i \phi}$ and the negative frequency points were multiplied by $e^{-i \phi}$ where $\phi$ is a random phase from 0 to $2 \pi$. This procedure is used in [Ebisuzaki, 1997].

In this study the numerical simulations are performed with a sampling rate of 5 seconds. The simulated noise must be generated for at least one month. The computational time for the IFFT algorithm is minimized when the number of samples is a power 
of 2 so $N$ is selected to be the smallest power of two such that the time series is at least one month long.

The two dimensional discrete Fourier transform is defined as

$$
\begin{array}{r}
X\left[l_{1}, l_{2}\right]=\sum_{n_{2}=0}^{N_{2}-1} \sum_{n_{1}=0}^{N_{1}-1} x\left[n_{1}, n_{2}\right] e^{-i 2 \pi\left(l_{1} n_{1} / N_{1}+l_{2} n_{2} / N_{2}\right)} \\
\text { where } \quad l_{1}=0,1,2, \ldots, N_{1}-1 ; \quad l_{2}=0,1,2, \ldots, N_{2}-1 .
\end{array}
$$

The above equation is utilized in Section 2.6 for analyzing the spatial frequencies of regional gravity plots. The result of Eq. (B.10) is used in Eq. (C.3) to determine the two dimensional power spectral density. 


\section{Appendix C}

\section{Spectral Density}

The total power of a continuous time series $x(t)$ is defined by:

$$
P=\int_{-\infty}^{\infty}|X(f)|^{2} d f
$$

where $X(f)$ is the Fourier transform of $x(t)$ and is given by Eq. (B.1). The power spectral density (PSD) is a representation of the power of a signal over a certain frequency bandwidth and is labeled $u n i t^{2} / H z$. The PSD for each discrete frequency value $f$ can be written:

$$
P S D(f)=|X(f)|^{2}+|X(-f)|^{2}, \quad f \geq 0 .
$$

If the time series $x(t)$ is real Eq. (C.2) simplifies to:

$$
P S D(f)=2|X(f)|^{2}, \quad f \geq 0 .
$$

The plots describing the satellite-to-satellite measurement errors presented in Section 4.2 are given in terms of the amplitude spectral density (ASD) which is the square-root of the PSD having the label unit $/ \sqrt{H z}$. 


\section{Appendix D}

\section{Satellite-to-Satellite Range and Range-rate Equations}

\section{D.1 Tracking Observables}

The primary observable of the GRACE mission is the satellite-to-satellite range and range-rate measurements made by the K-band microwave ranging system. Simulations show that the best results are obtained when using the range-rate measurements [Kim, 2000] and that is the convention in current GRACE data processing. Both range and range-rate equations are developed in this section.

The satellite-to-satellite range is defined by

$$
\rho=\sqrt{\left(\mathbf{r}_{1}-\mathbf{r}_{2}\right)^{T}\left(\mathbf{r}_{1}-\mathbf{r}_{2}\right)}
$$

where $\mathbf{r}_{1}$ and $\mathbf{r}_{2}$ are the inertial position vectors of satellite 1 and satellite 2 respectively. The vector $\mathbf{r}_{12}$ defines the difference between the two satellite position vectors:

$$
\mathbf{r}_{12}=\mathbf{r}_{1}-\mathbf{r}_{2}
$$

and the line-of-sight unit vector $\hat{\mathbf{e}}_{12}$ is defined by

$$
\hat{\mathbf{e}}_{12}=\frac{\mathbf{r}_{12}}{\rho} .
$$

Differentiating the range observable gives the range-rate

$$
\dot{\rho}=\dot{\mathbf{r}}_{12} \cdot \hat{\mathbf{e}}_{12} .
$$


The range-rate observable is a projection of the velocity difference along the line-of-sight unit vector and differs in magnitude from the velocity difference vector.

\section{D.2 Measurement Partial Derivatives}

The partial derivatives of the range and range-rate observables with respect to the satellite position and velocity vectors are given here. These partials are contained in the $H$ matrix of Eq. (3.17).

The partial derivatives of the range observable are

$$
\begin{aligned}
\frac{\partial \rho}{\partial \mathbf{r}_{1}} & =\left(\frac{\mathbf{r}_{1}-\mathbf{r}_{2}}{\rho}\right)^{T} & \frac{\partial \rho}{\partial \mathbf{r}_{2}} & =-\frac{\partial \rho}{\partial \mathbf{r}_{1}} \\
\frac{\partial \rho}{\partial \mathbf{v}_{1}} & =0 & \frac{\partial \rho}{\partial \mathbf{v}_{2}} & =0
\end{aligned}
$$

The partial derivatives of the range-rate observable are

$$
\begin{aligned}
\frac{\partial \dot{\rho}}{\partial \mathbf{r}_{1}} & =\frac{1}{\rho}\left(\dot{\mathbf{r}}_{12}-\frac{\mathbf{r}_{12}}{\rho} \dot{\rho}\right)^{T} & \frac{\partial \dot{\rho}}{\partial \mathbf{r}_{2}} & =-\frac{\partial \dot{\rho}}{\partial \mathbf{r}_{1}} \\
\frac{\partial \dot{\rho}}{\partial \mathbf{v}_{1}} & =\left(\frac{\mathbf{r}_{12}}{\rho}\right)^{T} & \frac{\partial \dot{\rho}}{\partial \mathbf{v}_{2}} & =-\frac{\partial \dot{\rho}}{\partial \mathbf{v}_{1}}
\end{aligned}
$$

\section{D.3 Perturbed Motion Equations}

This section develops a simplified range-rate perturbation equation due to perturbed satellite motion. The resulting equation is applied using Hill's equations in Appendix E. The purpose of this method is to include the accelerometer errors into the satellite-to-satellite range-rate observations. This procedure is developed by [Kim, 2000].

The nominal satellite-to-satellite range $\rho_{0}$ is defined by

$$
\rho_{0}=\left(\mathbf{r}_{10}-\mathbf{r}_{20}\right) \cdot \hat{\mathbf{e}}_{0}
$$


where $\mathbf{r}_{10}$ and $\mathbf{r}_{20}$ are the nominal position vectors of satellites 1 and 2 respectively and $\hat{\mathbf{e}}_{0}$ is the nominal line-of-sight unit vector. The perturbed range $\rho$ is then defined by

$$
\rho=\left(\mathbf{r}_{1}-\mathbf{r}_{2}\right) \cdot \hat{\mathbf{e}}
$$

where

$$
\begin{gathered}
\mathbf{r}_{1}=\mathbf{r}_{10}+\delta \mathbf{r}_{1} \\
\mathbf{r}_{2}=\mathbf{r}_{20}+\delta \mathbf{r}_{2} \\
\hat{\mathbf{e}}=\hat{\mathbf{e}}_{0}+\delta \hat{\mathbf{e}}
\end{gathered}
$$

and $\delta \mathbf{r}_{1}, \delta \mathbf{r}_{2}$, and $\hat{\mathbf{e}}_{0}$ are the perturbed position and line-of-sight vectors. Combining Eq. (D.11) and Eq. (D.10) and considering the definition of Eq. (D.9), and assuming $\delta \hat{\mathbf{e}}$ is close to zero, the perturbed range equation is

$$
\delta \rho=\left(\delta \mathbf{r}_{1}-\delta \mathbf{r}_{2}\right) \cdot \hat{\mathbf{e}}_{0} .
$$

This is the projection of the relative position vector variation onto the line-of-sight unit vector. Similarly, the perturbed range-rate equation is

$$
\begin{aligned}
\delta \dot{\rho} & =\left(\delta \dot{\mathbf{r}}_{1}-\delta \dot{\mathbf{r}}_{2}\right) \cdot \hat{\mathbf{e}}_{0} \\
& +\frac{1}{\rho_{0}}\left(\dot{\mathbf{r}}_{10}-\dot{\mathbf{r}}_{20}\right) \cdot \hat{\mathbf{f}}_{0}\left(\delta \mathbf{r}_{10}-\delta \mathbf{r}_{20}\right) \cdot \hat{\mathbf{f}}_{0} \\
& +\frac{1}{\rho_{0}}\left(\dot{\mathbf{r}}_{10}-\dot{\mathbf{r}}_{20}\right) \cdot \hat{\mathbf{g}}_{0}\left(\delta \mathbf{r}_{10}-\delta \mathbf{r}_{20}\right) \cdot \hat{\mathbf{g}}_{0}
\end{aligned}
$$

where $\hat{\mathbf{e}}_{0}, \hat{\mathbf{f}}_{0}$, and $\hat{\mathbf{g}}_{0}$ are orthonormal and $\hat{\mathbf{f}}_{0}$ is in the cross-track direction. By assuming that the satellites are in a circular tandem orbit the second term becomes zero, and since the value of $\rho_{0}$ is relatively large the third term can be neglected. The simplified expression for the range-rate perturbation caused by a perturbed satellite motion is then

$$
\delta \dot{\rho}=\left(\delta \dot{\mathbf{r}}_{1}-\delta \dot{\mathbf{r}}_{2}\right) \cdot \hat{\mathbf{e}}_{0}
$$


The range and range-rate perturbed motion equations are now developed in the satellite radial, transverse, and normal local coordinate frame as shown in Figure D.1. For the local coordinates $\hat{\mathbf{h}}_{i j}$, the subscript $i$ describes the directional component $(u, v, w)$, and $j$ is the satellite number.

The range equation can described by

$$
\delta \rho=\left(\sum_{i=u, v, w}\left(\delta x_{i}\right)_{1} \hat{\mathbf{h}}_{i 1}-\sum_{i=u, v, w}\left(\delta x_{i}\right)_{1} \hat{\mathbf{h}}_{i 2}\right) \cdot \hat{\mathbf{e}}_{0}
$$

Again, assuming circular tandem satellite orbits, Eq. (D.15) is simplified to

$$
\delta \rho=\left(u_{1}+u_{2}\right) \sin \frac{\theta}{2}+\left(v_{1}-v_{2}\right) \cos \frac{\theta}{2}
$$

where $\theta$ is the angle of separation between the two satellites and $u_{i}$ and $v_{i}$ are the perturbed positions from the nominal circular orbit. The normal terms are zero since a circular orbit is assumed. Similarly, the range-rate perturbed motion equation is

$$
\delta \dot{\rho}=\left(\dot{u}_{1}+\dot{u}_{2}\right) \sin \frac{\theta}{2}+\left(\dot{v}_{1}-\dot{v}_{2}\right) \cos \frac{\theta}{2}
$$

where $\dot{u}_{i}$ and $\dot{v}_{i}$ are the perturbed velocities from the nominal circular orbit. Eq. (D.17) is applied to Hill's equations in the next section to provide a means of converting accelerometer errors into range-rate observation errors. 


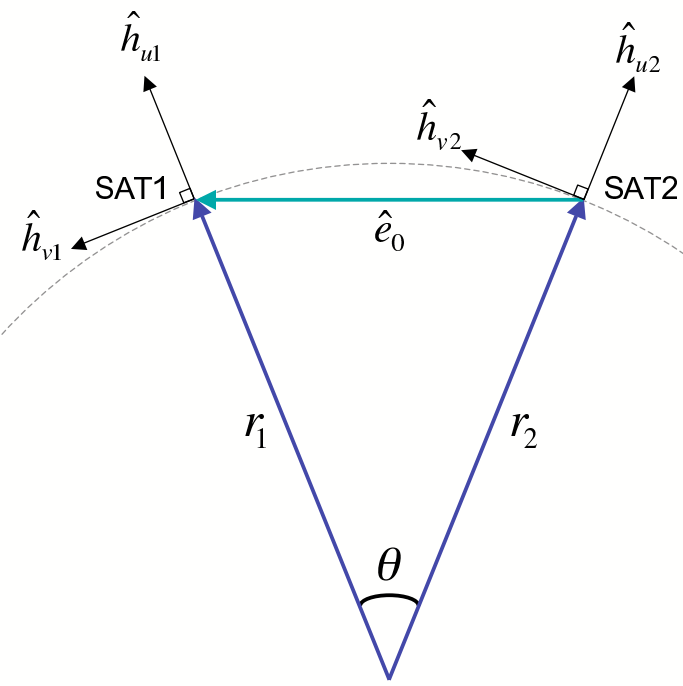

Figure D.1: Local coordinates. 


\section{Appendix E}

\section{Application of Hill's Equations}

This section summarizes the use of Hill's equations to determine a transfer function between a perturbation on the satellite motion and the perturbation in the rangerate. This procedure allows the power spectral density which describes the accelerometer errors to be mapped into a power spectral density of the associated range-rate perturbation. The procedure outlined here is developed by [Kim, 2000].

The Hill's equations $[$ Hill, 1878] describe relative satellite motion in the local radial, transverse, and normal coordinate system. The solution for a near-circular orbit is given by

$$
\begin{aligned}
& f_{u}=\ddot{u}-2 n \dot{v}-3 n^{2} u \\
& f_{v}=\ddot{v}+2 n \dot{u} \\
& f_{w}=\ddot{w}+n^{2} w
\end{aligned}
$$

where $n=\sqrt{\mu / r_{0}^{3}}$ is the mean motion of the satellite, and $u, w$, and $v$ are the perturbed positions and $f_{u}, f_{v}$, and $f_{w}$ are the disturbing forces in the radial, normal, and transverse directions.

Considering Eq.(D.17) and Eq. (E.1) the relationship between the perturbed 
satellite state and the perturbed range-rate can be described in state-space form:

$$
\begin{aligned}
& \dot{\mathbf{x}}=A \mathbf{x}+B \mathbf{f} \\
& \mathbf{y}=D \mathbf{x}
\end{aligned}
$$

where

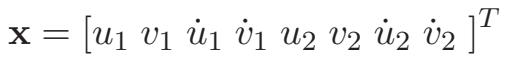

$$
\begin{aligned}
& \mathbf{f}=\left[\begin{array}{llll}
f_{u_{1}} & f_{v_{1}} & f_{u_{2}} & f_{v_{2}}
\end{array}\right]^{T}
\end{aligned}
$$

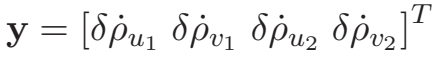

and $\mathbf{x}$ is the perturbed satellite state vector which describes both satellites, $\mathbf{f}$ is the perturbing force vector acting on each satellite, and $\mathbf{f}$ is the range-rate perturbation vector. The normal components are not included due to the fact that there are no normal terms in Eq. (D.17).

Expanding Eq. (E.2) gives

$$
\left[\begin{array}{c}
\dot{u}_{1} \\
\dot{v}_{1} \\
\ddot{u}_{1} \\
\ddot{v}_{1} \\
\dot{u}_{2} \\
\dot{v}_{2} \\
\ddot{u}_{2} \\
\ddot{v}_{2}
\end{array}\right]=\left[\begin{array}{cccccccc}
0 & 0 & 1 & 0 & 0 & 0 & 0 & 0 \\
0 & 0 & 0 & 1 & 0 & 0 & 0 & 0 \\
3 n^{2} & 0 & 0 & 2 n & 0 & 0 & 0 & 0 \\
0 & 0 & -2 n & 0 & 0 & 0 & 0 & 0 \\
0 & 0 & 0 & 0 & 0 & 0 & 1 & 0 \\
0 & 0 & 0 & 0 & 0 & 0 & 0 & 1 \\
0 & 0 & 0 & 0 & 3 n^{2} & 0 & 0 & 2 n \\
0 & 0 & 0 & 0 & 0 & 0 & -2 n & 0
\end{array}\right] \cdot\left[\begin{array}{c}
u_{1} \\
v_{1} \\
\dot{u}_{1} \\
\dot{v}_{1} \\
u_{2} \\
v_{2} \\
\dot{u}_{2} \\
\dot{v}_{2}
\end{array}\right]+\left[\begin{array}{llll}
0 & 0 & 0 & 0 \\
0 & 0 & 0 & 0 \\
1 & 0 & 0 & 0 \\
0 & 1 & 0 & 0 \\
0 & 0 & 0 & 0 \\
0 & 0 & 0 & 0 \\
0 & 0 & 1 & 0 \\
0 & 0 & 0 & 1
\end{array}\right] \cdot\left[\begin{array}{c}
f_{u_{1}} \\
f_{v_{1}} \\
f_{u_{2}} \\
f_{v_{2}}
\end{array}\right]
$$


Expanding Eq. (E.3) gives

$$
\left[\begin{array}{c}
\delta \dot{\rho}_{u_{1}} \\
\delta \dot{\rho}_{v_{1}} \\
\delta \dot{\rho}_{u_{2}} \\
\delta \dot{\rho}_{v_{2}}
\end{array}\right]=\left[\begin{array}{cccccccc}
0 & 0 & k_{1} & 0 & 0 & 0 & 0 & 0 \\
0 & 0 & 0 & k_{2} & 0 & 0 & 0 & 0 \\
0 & 0 & 0 & 0 & 0 & 0 & k_{1} & 0 \\
0 & 0 & 0 & 0 & 0 & 0 & 0 & -k_{2}
\end{array}\right] \cdot\left[\begin{array}{c}
u_{1} \\
v_{1} \\
\dot{u}_{1} \\
\dot{v}_{1} \\
u_{2} \\
v_{2} \\
\dot{u}_{2} \\
\dot{v}_{2}
\end{array}\right]
$$

where

$$
\begin{aligned}
& k_{1}=\sin \frac{\theta}{2} \\
& k_{2}=\cos \frac{\theta}{2}
\end{aligned}
$$

A transfer function with zero initial conditions is given by

$$
G(s)=D(s I-A)^{-1} B
$$

The amplitude spectral density for the accelerometer measurement errors given in Table 4.1. The resulting range-rate perturbation amplitude spectral density is the sum of the radial and transverse perturbations and is shown in Figure (4.3). The IFFT is taken of this amplitude spectral density to produce a time-series of range-rate observation errors which are then included in the simulation to account for errors in the accelerometer. The IFFT procedure is summarized in Appendix B. 